\title{
Multi-scale Modelling of Hydrogen Diffusion in Solids: Impact of Diffusion on Denuded Zones in Zr Alloy Pressure Vessels and Hydrogen Diffusion in Lead Zirconate Titanate
}

\author{
by \\ Pandula Manura Liyanage \\ A dissertation submitted to the \\ Faculty of Graduate and Postdoctoral Affairs \\ in partial fulfillment of the requirements for the degree of
}

Doctor of Philosophy in Civil Engineering

Ottawa-Carleton Institute for Civil Engineering

Department of Civil and Environmental Engineering

Carleton University

Ottawa, Ontario

February, 2020

(C) Copyright

Pandula Manura Liyanage, 2020 
The undersigned hereby recommends to the

Faculty of Graduate and Postdoctoral Affairs acceptance of the dissertation

\title{
Multi-scale Modelling of Hydrogen Diffusion in Solids: Impact of Diffusion on Denuded Zones in Zr Alloy Pressure Vessels and Hydrogen Diffusion in Lead Zirconate Titanate
}

\author{
submitted by Pandula Manura Liyanage \\ in partial fulfillment of the requirements for the degree of \\ Doctor of Philosophy in Civil Engineering
}

Professor R. K. N. D. Rajapakse, Thesis Supervisor

Professor Ronald E. Miller, Thesis Co-supervisor

Professor Yasser Hassan, Chair,

Department of Civil and Environmental Engineering

Ottawa-Carleton Institute for Civil Engineering

Department of Civil and Environmental Engineering

Carleton University

February, 2020 


\section{Abstract}

Zirconium alloys are extensively used as cladding material in nuclear reactors. They are vulnerable to hydrogen degradation under the harsh service conditions of the reactors, which necessitates continuous monitoring for the hydride content. The presence of hydride denuded zones in the latter stages of the pressure tube's life hinders the monitoring process, which is carried out by scrape samples taken from the surface of pressure tubes. A hypothesis is proposed that oxygen slows the diffusion of hydrogen and thereby encourages the occurrence of hydride denuded zones.

We Initially developed an approach which is used to determine the diffusivity of hydrogen in the hexagonal close packed (hcp) zirconium crystal, using a combination of first principles calculations and kinetic Monte Carlo (KMC) simulations. Rate constants found through the energy landscapes of hydrogen motion between different interstitial sites in the zirconium lattice, were used in KMC to determine the values of bulk diffusivity. Using previous research, we validated the model. We also found that hydrogen diffusivity in $\alpha$-Zr is closely isotropic, with a slightly higher diffusivity in the $\mathrm{C}$-axis direction. The individual diffusion jumps were closely investigated to identify the reasons for the isotropic nature of the diffusivity in the anisotropic hcp Zr lattice.

With the validated model we found that oxygen indeed decreases the diffusivity of hydrogen in Zr for moderate oxygen concentrations. We investigated the diffusion processes of individual $\mathrm{H}$ atoms, which showed that the reduction in diffusivity is caused by a decrease in the hopping rates and the formation of hydrogen traps by the combination of several interstitial sites. Though the diffusivity of $\mathrm{H}$ seems to be reduced by $\mathrm{O}$, by looking at the $\mathrm{O}$ concentration profiles found in $\mathrm{Zr}$ alloy pressure tubes, we see that the slowing down is insufficient to cause a significant enough change to the size of hydride precipitates. This causes us to reject the hypothesis as the main reason for the formation of denuded zones in Zircaloy pressure tubes.

Interested by the effects of stress on diffusion, we used the model to determine 
the effect on the diffusivity of $\mathrm{H}$ in Zr by applied hydrostatic stresses. With this, we see a decrease in the diffusivity with increase in compressive stress showing us that stress does have an effect on the diffusivity of $\mathrm{H}$. The main reason for the decrease in diffusivity is the increase in activation barriers, especially from an octahedral site, due to the preference for it with increasing compressive stresses. We intend to extend the results of the study to look at the significance of the variation in stress on diffusion behavior of $\mathrm{H}$.

Lead Zirconate Titanate (PZT) is a widely used piezoelectric and ferroelectric material with applications ranging from actuators in fuel injectors to ferroelectric random-access memories. PZT is also affected by $\mathrm{H}$ absorption, not only by causing embrittlement, but also reduction in polarity and $\mathrm{Pb}$ migration. This gives rise to the need of understanding the behavior of hydrogen in piezoelectric material. This research presents the initial results of a study which aims to simulate hydrogen diffusion in PZT using the validated diffusion model used for Zr. First-principles calculations were done to determine the possible hydrogen occupancy locations in the PZT lattice. For these locations we calculated the effect on polarization and the dimensions of the PZT lattice by dissolved hydrogen and predicting paths available for the escape of hydrogen atoms. These results will serve as a basis for future nudged elastic band calculations to determine the diffusion characteristics for the individual diffusion steps, which can in turn be used to predict the bulk diffusion characteristics with Kinetic Monte Carlo (KMC) simulations. 
I would like to dedicate my thesis to my parents who have been supporting and encouraging me through out my studies 


\section{Acknowledgments}

First and foremost, I would like to present my sincere gratitude to my supervisors, Prof. R. K. N. D. Rajapakse and Prof. Ronald Miller for introducing this research topic to me, providing guidance, support, advice, encouragement, and friendship throughout the research.

I am also thankful to my parents and my brother for showing enthusiasm at my work and providing the required support and encouragement during the progression of the research.

I would like to extend my thanks to the examination committee for the valuable comments and discussion. I am also grateful for the decision to nominate me for a Carleton University Medal.

I am grateful for the funding from Carleton University and NSERC's Discovery Grants program which was essential to realize this research. I would also like to acknowledge the support provided by Compute Canada through providing the computational resources necessary for the research.

Special thanks should be extended to Prod. Glenn McRae of the Department of Mechanical and Aerospace Engineering department of Carleton University, who was instrumental in instigating the thesis topic and the comments and suggestions provided throughout the research.

I would also like to thank my colleagues at Carleton University for the numerous interesting and helpful discussions held. I would like to extend my gratitude for the authors of the literature referred, which contributed to a major part of this research. 


\section{Table of Contents}

Abstract $\quad$ iii

Acknowledgments $\quad$ vi

Table of Contents vii

List of Tables $\quad$ xi

List of Figures $\quad$ xii

$\begin{array}{ll}\text { Nomenclature } & \text { Xv }\end{array}$

List of Symbols $\quad$ xvii

1 Introduction and background 1

1.1 Motivation for Research . . . . . . . . . . . . . . . . 2

1.2 Literature Review . . . . . . . . . . . . . . . . . . . . . . 4

1.2.1 Zirconium Alloys .................. 4

1.2.2 Hydrogen Embrittlement . . . . . . . . . . . . . . . 5

1.2.3 Hydrogen in Zirconium . . . . . . . . . . . . . . . . 8

1.2.4 Oxygen in Zirconium . . . . . . . . . . . . . . 16

1.2.5 Hydride Denuded Zones . . . . . . . . . . . . . . . 18

1.2.6 Lead Zirconate Titanate . . . . . . . . . . . . . . . . 20

1.3 Scope and Objectives . . . . . . . . . . . . . . . . . . . 21

1.4 Publications . . . . . . . . . . . . . . . . . . . 22

1.4.1 Journal Papers . . . . . . . . . . . . . . . . . . . . . . 22

1.4.2 Conference presentations . . . . . . . . . . . . . . 23

1.5 Organization of the Thesis . . . . . . . . . . . . . 23 
Bibliography ............................. 24

2 Diffusion Theory and Computational Methods 30

2.1 Diffusion of Solids . . . . . . . . . . . . . . . . . . . 30

2.1 .1 Fick's Law . . . . . . . . . . . . . . . . . . . . 31

2.1.2 Random Walk Theory . . . . . . . . . . . . . . . . . 33

2.1 .3 Transition States . . . . . . . . . . . . . . . . . . 37

2.1.4 Arrhenius Relationship . . . . . . . . . . . . . . . . . 38

2.2 Computational Methods . . . . . . . . . . . . . . . . . . . . . 39

2.2.1 Density Functional Theory . . . . . . . . . . . . . . . . . 39

2.2.2 Nudged Elastic Band Method . . . . . . . . . . . . . . . 41

2.2.3 Vienna Ab-initio Simulation Package . . . . . . . . . . . . 41

2.2.4 Kinetic Monte Carlo Simulation . . . . . . . . . . . . . . 42

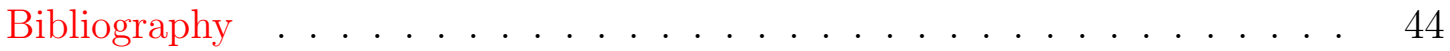

3 Multi-scale approach for determining hydrogen diffusivity in zirconium $\quad 47$

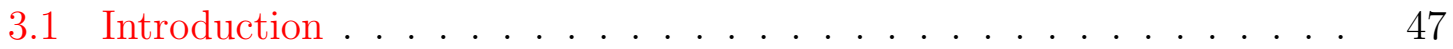

3.2 Hydrogen in Zirconium . . . . . . . . . . . . . . . . . . . . 48

3.3 Hydrogen Diffusion in Zirconium . . . . . . . . . . . . . . . . 49

3.4 Modelling Approach . . . . . . . . . . . . . . . . . . . 52

3.4.1 First Principles Studies . . . . . . . . . . . . . . . 55

3.4.2 Kinetic Monte Carlo Simulation . . . . . . . . . . . . . 56

3.4.3 Pseudo Energy Basin Effect . . . . . . . . . . . . . . . 58

3.5 Results and Discussion . . . . . . . . . . . . . . . 61

3.5.1 Rate Constants . . . . . . . . . . . . . . . . . 61

3.5.2 Modification for the Pseudo-Basin . . . . . . . . . . . . 63

3.5.3 Diffusivity of Hydrogen in Zirconium . . . . . . . . . . . 65

3.5 .4 Modelling Process . . . . . . . . . . . . . . . . . . . . 68

3.5.5 Further Discussion . . . . . . . . . . . . . . . 70

3.6 Conclusion and Future Directions . . . . . . . . . . . . 70

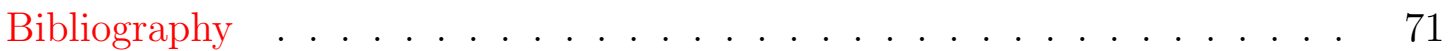


4 Denuded Zones in Zirconium Pressure Vessels: Oxygen's Role Examined via Multi-scale Diffusion Model 76

4.1 Introduction . . . . . . . . . . . . . . . . . . . 77

4.2 Computational Details . . . . . . . . . . . . . . 78

4.3 Results and Discussion . . . . . . . . . . . . . . . . . 79

4.3.1 Hydrogen diffusion in Pure $\mathrm{Zr}$. . . . . . . . . . . . . . . 80

4.3.2 Hydrogen diffusion in $\mathrm{Zr}$ with interstitial oxygen . . . . . . . . 83

4.3.3 Implications of the Diffusivity on Hydride Precipitation . . . . 92

4.4 Conclusions and Future Direction . . . . . . . . . . . . . . . . . . . . 94

Bibliography . . . . . . . . . . . . . . . . . . 95

5 Effect of Stress on Hydrogen Diffusion in Zirconium, investigated $\begin{array}{ll}\text { through multi-scale methods } & 98\end{array}$

5.1 Introduction . . . . . . . . . . . . . . . . . . . . . . . 99

5.2 Computational Method . . . . . . . . . . . . . . . . . 100

5.3 Results and Discussion . . . . . . . . . . . . . . . . . . . 102

5.3.1 Variation of pressure . . . . . . . . . . . . . . . . 102

5.3.2 First principles calculations . . . . . . . . . . . . . . . 104

5.3 .3 Diffusivity of $\mathrm{H}$ in $\mathrm{Zr}$. . . . . . . . . . . . . . . . . . . . . . . 109

5.4 Conclusions and Future Directions . . . . . . . . . . . . . . 112

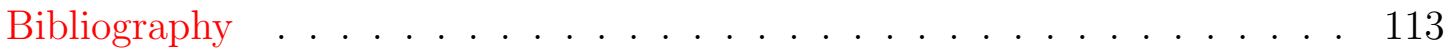

6 First-principles study of hydrogen in lead zirconate titanate $\quad 116$

6.1 Introduction . . . . . . . . . . . . . . . . . . . 117

6.1.1 Motivation of the study . . . . . . . . . . . . . . 117

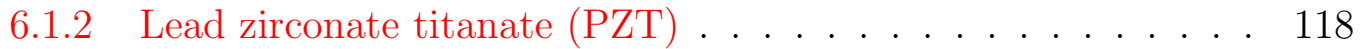

6.1.3 Hydrogen degradation of PZT . . . . . . . . . . . . . . . . . 122

6.1.4 Mechanisms of hydrogen degradation . . . . . . . . . . . . 122

6.1.5 Hydrogen diffusion and occupancy . . . . . . . . . . . . . 123

6.2 Research Methodology . . . . . . . . . . . . . . . 124

6.2.1 Crystal structure of PZT . . . . . . . . . . . . . . 125

6.2.2 Probable hydrogen occupancy locations . . . . . . . . . . . . . 127

6.3 Results and Discussion . . . . . . . . . . . . . . . . . . . . . 127

6.3.1 PZT crystal structure . . . . . . . . . . . . . . . . 127

6.3.2 Hydrogen occupancy locations . . . . . . . . . . . . . . . . . 128 
6.3.3 Escape paths for hydrogen diffusion . . . . . . . . . . . . . 133

6.4 Conclusion and Future Directions . . . . . . . . . . . . . . . . 133

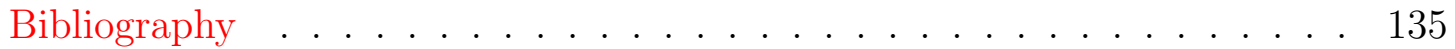

7 Conclusion and Future work $\quad 140$

7.1 Summary and Conclusions . . . . . . . . . . . . . . . . . 140

7.2 Future work . . . . . . . . . . . . . . . . . . . . 142

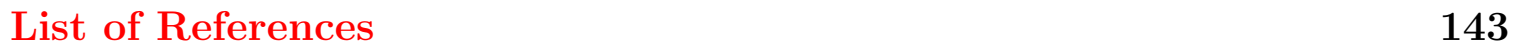

Appendix A Semi-Classical Harmonic Transition State Theory 156

Bibliography . . . . . . . . . . . . . . . . 157

$\begin{array}{lll}\text { Appendix B Absorbing Markov Chains } & 158\end{array}$

Bibliography ....................... 159 


\section{List of Tables}

1.1 Composition of widely used zirconium alloys . . . . . . . . 6

1.2 Coefficients for solubility of $\mathrm{H}$ in $\mathrm{Zr} \ldots \ldots \ldots \ldots$

$1.3 \mathrm{H}$ solubility for reactor conditions . . . . . . . . . . . . . 10

1.4 Crystal details of zirconium hydrides . . . . . . . . . . . 13

1.5 Diffusion coefficients from literature for $\mathrm{H}$ in $\mathrm{Zr} \ldots \ldots \ldots \ldots$

1.6 Activation energies for transition steps of $\mathrm{H}$ diffusion in $\mathrm{Zr} \ldots \ldots .16$

1.7 Diffusion coefficients $\mathrm{O}$ in $\mathrm{Zr}$ available in literature $\ldots \ldots \ldots . . .217$

$3.1 D_{0}$ and $E_{a}$ for $\mathrm{H}$ diffusion in $\mathrm{Zr} \ldots \ldots \ldots \ldots$

3.2 Escape paths for $\mathrm{H}$ in $\mathrm{Zr} \ldots \ldots \ldots \ldots \ldots \ldots$

3.3 Comparison of diffusivity for pseudo energy basin . . . . . . . . . 59

3.4 Paths for $\mathrm{H}$ diffusion in modified system . . . . . . . . . . 60

$3.5 \Gamma_{0}$ and $E_{a}$ for different diffusion steps $\ldots \ldots \ldots \ldots \ldots$

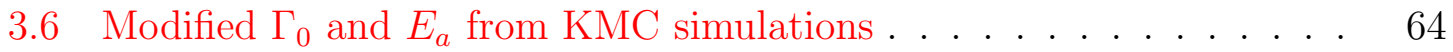

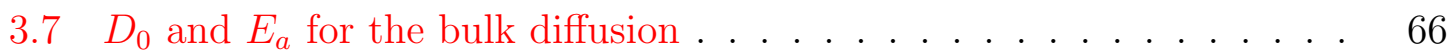

3.8 Probability of selecting an escape path . . . . . . . . 68

4.1 Comparison of activation energies for nearest neighbour transitions . 81

4.2 Vibration frequencies of each diffusion step . . . . . . . . . 81

4.3 Diffusivity parameters for $\mathrm{H}$ diffusion in $\mathrm{Zr} \ldots \ldots \ldots$. . . . . . 82

4.4 Diffusivity parameters in the basal plane direction . . . . . . . 90

4.5 Diffusivity parameters in the C-axis direction . . . . . . . . 90

5.1 Average pressure in systems with $\mathrm{H}$ used in the study . . . . . . . . 105

6.1 Lattice parameters of tetragonal PZT unit cell . . . . . . . . . . . 121

6.2 Fractional C-direction coordinates in PZT unit cell . . . . . . . . . 121

6.3 Lattice parameters for different functionals . . . . . . . . . . . 128

6.4 Fractional coordinates from different functionals . . . . . . . . . . 128

6.5 Relative total energy of the system with interstitial H . . . . . . . . 129

6.6 Variation of lattice parameters, volume, and polarization of PZT . . . 133 


\section{List of Figures}

1.1 Hydride denuded zones in zirconium reactor tubes . . . . . . . . . . 3

1.2 Crystal structure of hcp Zr lattice . . . . . . . . . . . . . . . . . 7

1.3 Variation of solubility of $\mathrm{H}$ in $\mathrm{Zr}$ with temperature . . . . . . . . . 11

1.4 Interstitial sites of Zr lattice . . . . . . . . . . . . . . . . . . 12

1.5 Microscopic view of a hydrided zircaloy tube $[43] \ldots \ldots$. . . . . . . 12

1.6 Phases of hydrogen in zirconium . . . . . . . . . . . . . . . . 13

1.7 Effect of cooling rate on denuded zone [63] . . . . . . . . . . . 19

1.8 Phase diagram of PZT . . . . . . . . . . . . . . . . . 20

1.9 Tetragonal crystal structure of PZT . . . . . . . . . . . . . . 21

2.1 Particle flow in Y-direction . . . . . . . . . . . . . . . . . . 32

2.2 Random walk of interstitial solutes . . . . . . . . . . . . . . 34

2.3 Diffusion of an interstitial atom . . . . . . . . . . . . . . . . 37

2.4 Potential energy landscape for the diffusion . . . . . . . . . . . . . 37

2.5 Energy landscape of a diffusion step . . . . . . . . . . . . . . . . 39

2.6 Illustration of picking the escape path . . . . . . . . . . . . . . 44

3.1 Phase diagram for hydrogen in zirconium . . . . . . . . . . . . . . 50

3.2 Hexagonal close packed crystal structure . . . . . . . . . . . . 51

3.3 Interstitial sites in zirconium unit cell . . . . . . . . . . . . . . . . . 52

3.4 Typical energy variation along the diffusion path . . . . . . . . . 56

3.5 Escape paths for $\mathrm{H}$ in tetrahedral sites . . . . . . . . . . . . . . 57

3.6 Escape paths for $\mathrm{H}$ in octahedral sites . . . . . . . . . . . . 58

3.7 Modified interstitial site arrangement . . . . . . . . . . . . . 60

3.8 Escape paths for $\mathrm{H}$ in octahedral sites in modified system . . . . . . . 61

3.9 Energy landscapes for different diffusion steps . . . . . . . . . . . . 62

3.10 Plot of the modified rate constants vs the temperature . . . . . . . 64

3.11 Comparison of diffusivities . . . . . . . . . . . . . . . 65

3.12 Bulk Diffusivity vs temperature . . . . . . . . . . . . . 66 
3.13 Anisotropy of bulk diffusion . . . . . . . . . . . . . 67

3.14 Modelling process followed in the current study . . . . . . . . . . 69

4.1 interstitial sites for $\mathrm{H}$ occupancy in $\mathrm{Zr} \ldots \ldots \ldots$. . . . . 80

4.2 Bulk diffusivity vs temperature from KMC . . . . . . . . . . . 82

4.3 Variation of index $i$ for different $\mathrm{O}$ concentrations . . . . . . . . 84

4.4 Variation of index $j$ for different $\mathrm{O}$ concentrations . . . . . . . 85

4.5 Variation of $\Delta E$ around a single $\mathrm{O}$ atom $\ldots \ldots \ldots \ldots$

4.6 Region affected by a single $\mathrm{O} \ldots \ldots \ldots \ldots \ldots$

4.7 Arrangement of neighbouring regions by $\mathrm{O}$ atoms $\ldots \ldots \ldots$

4.8 Variation of $\Delta E$ with multiple surrounding $\mathrm{O}$ atoms $\ldots \ldots \ldots$.

4.9 Variation of diffusivity with $\mathrm{O}$ concentration $\ldots \ldots \ldots \ldots$

4.10 Percentage change in diffusivity . . . . . . . . . . . . . 90

4.11 Trap formed by pseudo basin $\mathrm{T}_{2,1} \mathrm{~T}_{2,2}$ and $\mathrm{O}_{2,2}$ sites . . . . . . 91

4.12 Variation of $D^{1.5}$ with the temperature . . . . . . . . . . . 93

4.13 Relative size of the precipitates formed . . . . . . . . . . . 93

4.14 Oxygen concentration profile in Zirconium . . . . . . . . . . . 94

5.1 Unit cell for $\alpha \mathrm{Zr} \ldots \ldots \ldots$. . . . . . . . . . . . . . . . . 101

5.2 Locations available for $\mathrm{H}$ to occupy stably . . . . . . . . . . . . . 101

5.3 Available transition streps . . . . . . . . . . . . . . . . . . 103

5.4 Variation of lattice parameters against pressure . . . . . . . . 104

5.5 Pressure variation with added H . . . . . . . . . . . . 105

5.6 Change in energy preference for $\mathrm{H}$ occupancy . . . . . . . . 106

5.7 Change in activation energy for all steps . . . . . . . . . . . 107

5.8 Change in activation energy for different steps . . . . . . . . . . 108

5.9 Variation of diffusivity with pressure . . . . . . . . . . . 110

5.10 Variation of $\ln (D)$ vs $1 / T$ with pressure $\ldots \ldots \ldots \ldots \ldots$

5.11 Variation of activation energy $E_{a}$ with the pressure $\ldots \ldots \ldots . . .111$

5.12 Variation of pre-exponent $D_{0}$ with the pressure $\ldots \ldots \ldots \ldots$

5.13 Variation of anisotropy with pressure . . . . . . . . . . . . . 112

6.1 Arrangement of atoms in a PZT unit cell . . . . . . . . . . . . . 119

6.2 Polarization Hysteresis behavior of ferroelectric material . . . . . . 119

6.3 Phase diagram of $\mathrm{PZT} \ldots \ldots \ldots \ldots$

6.4 Arrangement of atoms in a tetragonal PZT unit cell . . . . . . . . . 121 
6.5 Hysteresis loops and variation of polarization of the PZT for different H concentrations . . . . . . . . . . . . . . . . . . . . 123

6.6 Locations for hydrogen occupancy from Aggarawal et al. [35] . . . . . 124

6.7 Locations for hydrogen occupancy from Alvine et al. [11] . . . . . . 125

6.8 Atomic arrangement used to verify the model . . . . . . . . . 126

6.9 Occupancy locations for hydrogen in the PZT . . . . . . . . 130

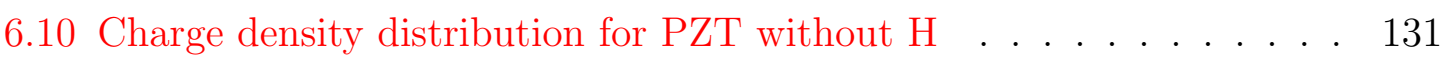

6.11 Charge density distribution for PZT with $\mathrm{H}$. . . . . . . . . . 132

6.12 Proposed escape paths available at each occupancy location . . . . . 134

B.1 Mean escape time from the pseudo-energy basin . . . . . . . . . 160

B.2 Probability of exiting along each path . . . . . . . . . . 160 


\section{Nomenclature}

\begin{tabular}{|c|c|}
\hline Acronym & Meaning \\
\hline bcc & Body centered cubic \\
\hline BWR & Boiling Water Reactors \\
\hline CANDU & Canada Deuterium Uranium \\
\hline $\mathrm{DFT}$ & Density Functional Theory \\
\hline $\mathrm{DHC}$ & Delayed Hydride Cracking \\
\hline fcc & Face centered cubic \\
\hline fct & face centered tetragonal \\
\hline hcp & Hexagonal close packed \\
\hline $\mathrm{HE}$ & Hydrogen Embrittlement \\
\hline HEDE & Hydrogen Enhanced Decohesion \\
\hline HELP & Hydrogen Enhanced Local Plasticity \\
\hline $\mathrm{HICE}$ & Hydrogen Internal Combustion Engines \\
\hline $\mathrm{KMC}$ & Kinetic Monte Carlo \\
\hline LWR & Light Water Reactors \\
\hline MPB & Morphological Phase Boundary \\
\hline NEB & Nudeged Elastic Band \\
\hline NN & Nearest neighbor \\
\hline PAW & projector-augmented wave \\
\hline
\end{tabular}




\begin{tabular}{cl}
\hline Acronym & \multicolumn{1}{c}{ Meaning } \\
\hline \hline PBE/PBE-GGA & Generalized gradient approximation \\
PT & of Perdew, Burke and Ernzerhof \\
PWR & Pressure Water Reactors \\
PZ & Lead Zirconate \\
PZT & Lead zirconate titanate \\
SC-HTST & Semi-Classical Harmonic Transition State Theory \\
TSSD & Total Solid Solubility in Dissolution \\
TSSP & Total Solid Solubility in Precipitation \\
VASP & Vienna Ab-initio Simulation Package \\
ZPE & Zero-point energy \\
\hline
\end{tabular}




\section{List of Symbols}

\begin{tabular}{|c|c|}
\hline Symbol & Meaning \\
\hline$a$ & Lattice parameter of a unit cell in the basal plane direction \\
\hline$c$ & Lattice parameter of a unit cell in the c-axis plane direction \\
\hline$C$ & Concentration \\
\hline$D$ & Diffusivity \\
\hline$D_{0}$ & Pre-exponent coefficient for hydrogen diffusivity in zirconium \\
\hline$E_{a}$ & Activation energy \\
\hline$\hat{H}$ & Hamiltonian operator \\
\hline$J$ & Diffusion flux \\
\hline$k_{B}$ & Boltzmann constant \\
\hline$S_{0}$ & Pre-exponent coefficient for hydrogen solubility in zirconium \\
\hline$S_{T}$ & Hydrogen solubility in zirconium in for a given temperature \\
\hline$T$ & Temperature(Absolute in most cases) \\
\hline$V$ & Partial molar volume of hydrogen in zirconium \\
\hline$\Gamma$ & Jump rate or hopping rate of a diffusion step \\
\hline$\nu$ & Oscillation frequency/vibration mode of an $\mathrm{H}$ atom \\
\hline$\sigma$ & Hydrostatic stress \\
\hline$\Psi$ & Quantum mechanical wave function \\
\hline
\end{tabular}




\section{Chapter 1}

\section{Introduction and background}

Nuclear energy is a clean and reliable source which has contributed to about $10.2 \%$ of the world electricity generation in 2017 [1]. There are 442 nuclear electricity generation plants around the world in 31 countries having a combined capacity of about 2563 $T W h$ (in 2018) [1,2]. Even though it is a cleaner, safer, and more reliable compared to most other energy sources, there is some resistance to maintaining nuclear power plants, concerning threats to people and the environment. The catastrophic nature in failure of power plants such as Fukushima Daiichi nuclear plant disaster caused by the Tsunami in 2011 has strengthened the arguments against nuclear power plants. The Three Mile Island power plant meltdown in 1979 and Chernobyl disaster in 1986 are other instances where nuclear power plants have failed. But the fact remains that, it is a cleaner energy source than most other popular commercial energy sources only producing $12 \mathrm{~g} \mathrm{CO}$ eq/ $k W h$ (measure of emission per unit power generated), whereas fossil gas and coal produces 490 and $820 \mathrm{~g} \mathrm{CO} \mathrm{CO}_{2} e q / k W h$ respectively [3]. Only onshore wind power has a cleaner record with an average emission of $11 \mathrm{~g}$ $\mathrm{CO}_{2} e q / k W h$, which is only slightly lower than the value for nuclear power. It also has the lowest fatality rate per energy unit produced [4]. Nuclear power is a major source for electricity generation in the world with 15 countries satisfying more than $20 \%$ of their electricity demand using nuclear energy in 2016 [2]. Canada is the 6th largest producer of nuclear power, generating about $4 \%$ (capacity of $14,071 \mathrm{MW}$ ) of the global nuclear power generation from 6 power plants [5]. There are even plans for NASA to build a nuclear power plant in Mars [6].

Nuclear power plants produce energy by nuclear fission reactions, where the nuclear fuel, radioactive Uranium pellets which are stored in small cylindrical containers submerged in water, break down with a release of neutrons, creating a chain reaction 
whilst producing a vast amount of energy as heat. This heat vaporises a coolant (water in most cases), which goes on to power the turbines to generate electricity. There are two main types of reactors based on how the heat energy produced is transmitted to the turbines [7].

- Pressure Water Reactors (PWR) - Pressurized and heated water from the reactor is used to heat and vaporize a secondary coolant in the electricity generator system, which goes on to power the turbines

- Boiling Water Reactors (BWR) - The coolant, water, is boiled from the heat generated from nuclear reactions and taken directly to generate power from the turbines

Of the nuclear reactors in the world, approximately $60 \%$ are PWRs and $21 \%$ are BWRs, which are collectively known as Light Water Reactors (LWR) $[7,8]$.

Zirconium alloys are extensively used as fuel cladding, pressure tubes, fuel channels, and fuel spacer grid material in LWRs [7,9,10]. In CANDU reactors, nuclear fuel is stored in cladding tubes made from Zr-2.5Nb (see Table 1.1) alloy which are $6 \mathrm{~m}$ long, $103 \mathrm{~mm}$ in diameter and have a wall thickness of $4.2 \mathrm{~mm}$ [11]. Low thermal neutron absorption, good mechanical properties, good corrosion resistance, apt thermal conductivity and thermal stability, and good creep behavior makes zirconium alloys excellent candidates for boundaries or barriers in nuclear plants [10,12-14].

\subsection{Motivation for Research}

While zirconium alloys have good corrosion resistance, the harsh conditions in nuclear reactors decompose water while forming a protective oxide layer with the release of hydrogen. Some of this $\mathrm{H}$ can get absorbed to $\mathrm{Zr}$ which make the reactor tubes vulnerable to failure. Delayed Hydride Cracking (DHC) and loss of toughness are the main mechanisms through which reactor tubes are found to fail due to $\mathrm{H}$ absorption. These mechanisms are not yet fully understood, but almost all research agrees that a critical hydride content is required for these types of failures to occur in Zr. Hence, the hydride content is continuously monitored to assess the safety of keeping the pressure tubes in service. The general mechanism of monitoring is by taking a scrape sample from the surface and determining its hydride content, assuming that the hydride content in the bulk is equivalent to that of the surface. 


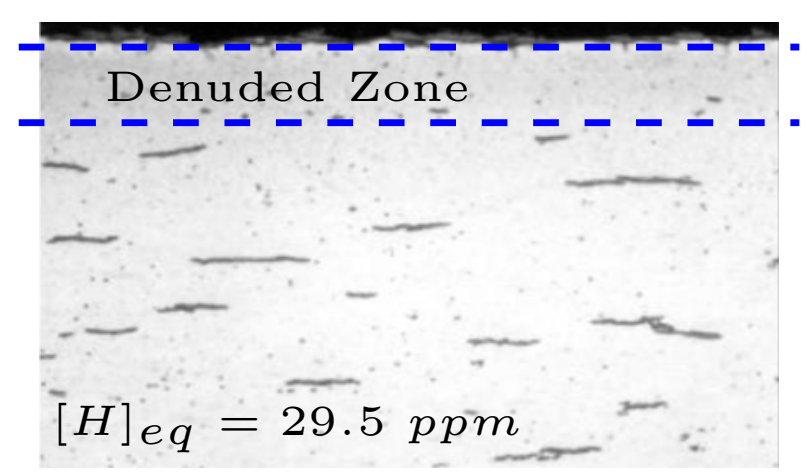

Figure 1.1: Hydride denuded zones in zirconium reactor tubes

From optical micrographs taken of some zirconium alloy tubes it is found that there exists a zone closer to the surface which has smaller sized hydrides than in the bulk (see Fig. 1.1). These zones are called "denuded zones" which mostly occur in old tubes with a relatively high hydride concentration. The presence of these denuded zones is problematic because they can invalidate the scrape sampling assumptions. Hardie [15] has suggested that these zones can be a result of slowing down of $\mathrm{H}$ diffusion due to the presence of $\mathrm{O}$ closer to the surface, which would prevent the formation of large hydride precipitates in the grain boundaries as they usually do. The hydrides formed will be smaller in size and intragranular in nature. Hence, we wanted to investigate the validity of the hypothesis and quantify the effects $\mathrm{O}$ has on diffusivity of $\mathrm{H}$.

Another hypothesis proposed as the reason for the formation of denuded zone is that the tensile stress exerted by the oxide at the metal-oxide interface attracts hydrogen close to it creating a hydrogen depleted region. Stresses found close to the oxide-metal interface are considerably high in the range of $1600-2500 \mathrm{MPa}$ [16-18]. Looking at the hypotheses provided as causes for denuded zones, we got interested in how stress is investigated in diffusion scenarios. In most studies we see the effect of stress is applied only thorough a stress gradient. Reading literature on stress based diffusion we found that diffusivity does changes with stress (significantly at high stress values). This made us want to look at how the diffusivity of $\mathrm{H}$ in $\mathrm{Zr}$ changes under applied hydrostatic stresses. Hence, we extended our study to look at how stress affects diffusivity and then look at to what extent this variation needs to considered in diffusion studies.

After developing the diffusion model and using it to investigate different problems 
related to $\mathrm{Zr}$ and denuded zones, we extended our study to include lead zirconate titanate (PZT), a material which is widely used as a piezoelectric material due to its superior dielectric and piezoelectric properties, such as high piezo electric charge coefficient, piezo electric voltage coefficient, high dielectric constant, high electromechanical coupling coefficient, high charge sensitivity, high energy density, and low mechanical loss [19-21]. PZT is used as actuators in fuel injectors of hydrogen engines. These actuators has advantages such as rapid response, precise positioning, improved fuel metering, variable stroke length and increased injection pressure capacity [22] over conventional electromagnetic solenoid technology. Due to contact with hydrogen fuel the performance of these actuators can degrade affecting its performance. While a lot of studies have been carried out on $\mathrm{H}$ degradation of PZT, we found very few studies which looked at $\mathrm{H}$ diffusivity, a very important property to understand the degradation effects in PZT. We wanted to look at the possibility of extending our study to use the same diffusion model to investigate $\mathrm{H}$ diffusion in PZT.

Based on the above discussion, the three main objectives of this study are,

- Effect of $\mathrm{O}$ on $\mathrm{H}$ diffusivity in $\mathrm{Zr}$ and, the validity of the hypothesis suggesting interstitial $\mathrm{O}$ close to the surface of pressure tubes cause denuded zones

- Effect of hydrostatic stress on $\mathrm{H}$ diffusivity in $\mathrm{Zr}$

- Determining H diffusivity in PZT

\subsection{Literature Review}

\subsubsection{Zirconium Alloys}

Zirconium is the 40th element of the periodic table having an electron configuration of $[\mathrm{Kr}] 4 d^{2} 5 s^{2}$ and an atomic mass of $91.22 \mathrm{amu}$. $\mathrm{Zr}$ is mainly used as barriers in nuclear and chemical plants due to the advantages mentioned earlier. Pure $\mathrm{Zr}$ solidifies from molten metal to a body centered cubic (bcc) structure ( $\beta$-phase) at around $2123 \mathrm{~K}\left(1850^{\circ} \mathrm{C}\right)$ and then transforms to a hexagonal close packed (hcp) structure ( $\alpha$-phase, see Fig. 1.2$)$ below $1135 K\left(862^{\circ} \mathrm{C}\right)$. Lattice parameters of the hcp $\mathrm{Zr}$ lattice $a$ and $c$ are found to be $3.23 \AA$ and $5.14 \AA$ respectively [23]. Pure $\mathrm{Zr}$ is more susceptible for corrosion, hence it is widely used as alloys, where as little as $0.5 \%$ of 
an alloying element can improve the corrosion resistance of Zr by a great deal [24]. Most commonly used alloying elements are [25]:

- Niobium $(\mathrm{Nb})$ - increases oxidation resistance, strength, and formability and decreases $\mathrm{H}$ pickup

- Tin (Sn) - increased corrosion resistance and counteracts the effects of nitrogen absorbed during corrosion

- Chromium (Cr) - improves corrosion resistance at lower quantities $(<1.6 \mathrm{wt} \%)$ while decreases the resistance at higher quantities

- Iron $(\mathrm{Fe})$ - Decreases the corrosion rate

- Nickel (Ni) - Improves the oxidation resistance, but increases the H pickup

Based on the used alloying elements, there are two main types of alloy systems [8]:

- Zircaloys - Sn based alloys mainly used by the United States

- Nb based alloys used by other countries such as Canada and Russia

Modern alloys such as ZIRLO ${ }^{T} M$ and $\mathrm{M}^{T} M$ has replaced some of the zircaloy reactor tubes. Table 1.1 shows the chemical composition of some of the widely-used zirconium alloys in nuclear reactors in the present and the past.

\subsubsection{Hydrogen Embrittlement}

Hydrogen $\left(\mathrm{H}_{2}\right.$ molecules $)$ cannot get readily absorbed to solid metals due to the presence of surface barriers. But in molten metal at high temperatures, $\mathrm{H}$ gets absorbed in large quantities. Once absorbed, hydrogen molecules disassociate into H atoms, giving them high transportation capabilities due to the smaller size. Absorption of $\mathrm{H}$ can produce problems to almost all metals causing a ductile to brittle transformation with a reduction of bearing capacity. This process is known as hydrogen embrittlement (HE). It has been found that HE transforms the ductile transgranular failure of metals to brittle intergranular failures [27,28]. Steel, nickel, aluminum, and zirconium are some of the metals which are highly susceptibility to HE and they have been 
Table 1.1: Composition of widely used zirconium alloys

\begin{tabular}{|c|c|c|c|c|c|c|}
\hline \multirow{2}{*}{ Alloy } & \multicolumn{6}{|c|}{ Composition of alloying elements (\%) } \\
\hline & $\mathrm{Sn}$ & $\mathrm{Nb}$ & $\mathrm{Fe}$ & $\mathrm{Cr}$ & $\mathrm{Ni}$ & $\mathrm{O}$ \\
\hline $\begin{array}{c}\text { Zircaloy-1 } \\
{[8,12,26]}\end{array}$ & $1.50-2.50$ & - & - & - & - & - \\
\hline $\begin{array}{c}\text { Zircaloy-2 } \\
{[8,12,26]}\end{array}$ & $1.20-1.70$ & - & $0.07-0.20$ & $0.05-0.15$ & $0.03-0.08$ & - \\
\hline $\begin{array}{c}\text { Zircaloy-3 } \\
{[12,26]}\end{array}$ & $0.25-0.50$ & - & $0.25-0.40$ & - & $( \pm 0.2)$ & - \\
\hline $\begin{array}{c}\text { Zircaloy-4 } \\
{[12,26]}\end{array}$ & $1.20-1.70$ & - & $0.18-0.24$ & $0.07-0.13$ & - & \\
\hline $\begin{array}{c}\mathrm{Zr}-2.5 \mathrm{Nb} \\
{[26]}\end{array}$ & - & 2.50 & - & - & - & - \\
\hline $\begin{array}{c}\mathrm{Zr}-1 \mathrm{Nb} \\
{[26]}\end{array}$ & - & 1.00 & - & - & - & - \\
\hline $\begin{array}{c}\text { ZIRLO }^{T M} \\
{[26]}\end{array}$ & 1.00 & 1.00 & 0.10 & - & - & - \\
\hline $\begin{array}{c}\mathrm{M} 5^{T M} \\
{[26]}\end{array}$ & -0 & 1.00 & - & - & - & 0.125 \\
\hline
\end{tabular}




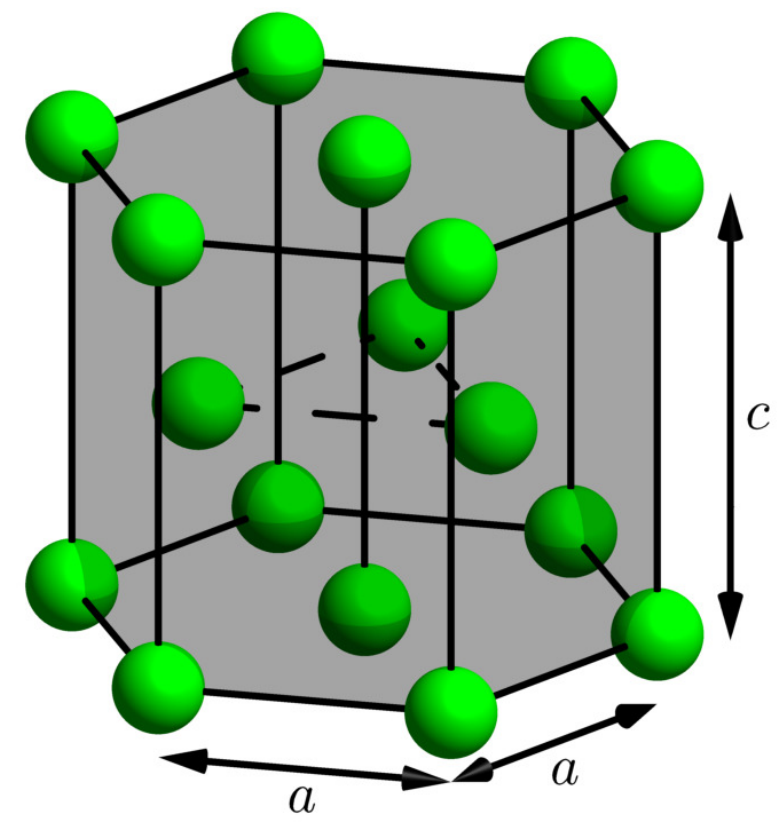

Figure 1.2: Crystal structure of hcp Zr lattice

widely investigated by researchers on studies related to HE. In metals such as nickel and steel, large substantial local plastic deformations can be observed even though the general failure mode appears to be a brittle [27].

Hydrogen can get absorbed to metals through a variety of methods. Disassociation of hydrogen molecules from electrochemical processes like corrosion, cathodic protection galvanic coupling, electroplating, electric discharge machining, and during materials processing and welding, and disassociated water vapor [29] can create $\mathrm{H}$ in atomic form, making is easier to get absorbed to metals.

HE has attracted wide attention in the research world, but even after a century of research the exact mechanisms for $\mathrm{HE}$ have not been fully understood. There are three main mechanisms which have been proposed as causes for HE, which may contribute to these failures by different levels [27, 30,31].

- Hydrogen Enhanced Decohesion (HEDE)

- Hydrogen Enhanced Local Plasticity (HELP)

- Formation of hydrides

Contribution of these mechanisms are expected to vary depending on the nature of the host lattice and other environmental conditions. HEDE suggests a lowering of the 
fracture energy due to the accumulation of $\mathrm{H}$ atoms at crack tips. This will encourage a cleavage like failure [31]. HELP is backed by the microscopy images showing high local plastic deformations and slip bands at crack surfaces [31]. Dislocations causes the ductile failures in metals by accumulating at crack tips and blunting them. It has been found that $\mathrm{H}$ will enhance the mobility of screw, edge and mixed dislocations in isolated and tangled forms at constant stresses [27,32], which supports the HELP mechanism. For hydride forming metals such as zirconium, niobium, and vanadium, the mechanism mainly responsible is found to be the formation of brittle hydrides [27], which have very low ductility and toughness similar to that of other brittle materials. A smaller quantity of hydrides has been observed even in non-hydride forming metals (Nickel) which has undergone HE. Though the exact mechanisms for HE are not yet realized, the fact that a minimum $\mathrm{H}$ content is required for the manifestation of $\mathrm{HE}$ has been agreed by everyone. For the current study the host lattice we use is $\mathrm{Zr}$, where the primary reason for HE is found to be the formation of brittle hydrides.

\subsubsection{Hydrogen in Zirconium}

\section{Hydrogen Pickup}

Due to the adverse effects of $\mathrm{H}$ absorption, measures are taken to keep $\mathrm{H}$ content to a minimum during manufacturing stages. Nonetheless, there are several mechanisms through which $\mathrm{H}$ can get picked up by the reactor tubes while in service.

- Oxidation reaction of zirconium with high temperature water,

$$
\mathrm{Zr}+2 \mathrm{H}_{2} \mathrm{O} \rightarrow \mathrm{ZrO}_{2}+2(1-x) \mathrm{H}_{2}+4 x \mathrm{H}
$$

produces a certain amount or radical or atomic $\mathrm{H}$. This $\mathrm{H}$ can get absorbed to the Zr lattice

- About $3-10 \mathrm{~cm}^{3} \mathrm{H}_{2} / \mathrm{kg}$ is added to collect the oxygen produced during the radiolytic decomposition of $\mathrm{D}_{2} \mathrm{O}$. Some of this can get absorbed to the reactor tubes [25]

- Nitrogen and carbon dioxide gases are added between the reactor tubes which could contain hydrogen as a impurity causing an $\mathrm{H}$ uptake [25] 
- Zirconium hydrides are used as moderators for nuclear reactors due to their high content of hydrogen, high neutron scattering cross-section, low neutron absorption cross-section, and negative temperature coefficient of reactivity. Hydrogen can escape from this zirconium hydrides at high temperatures (peak temperature of LWRs can reach $823 \mathrm{~K}$ ) and get absorbed to the reactor tubes [33]

For hydrogen to be picked up they need to be in atomic or radical form. Hydrogen molecules will generally not get absorbed due to the high surface barriers present [25]. The uptake of $\mathrm{H}$ is maximized in grain boundaries and places such as dislocations, which facilitate the formation of heterogeneous hydride compounds [32]. Formed zirconium oxides and zirconium hydrides can also act as barriers for hydrogen absorption [34].

\section{Solubility of Hydrogen}

Compared to $\mathrm{O}$, which has a solubility closer to $30 \mathrm{~atm} \%, \mathrm{H}$ has a very limited solubility in $\mathrm{Zr}$. The level of $\mathrm{H}$ solubility in $\mathrm{Zr}$ has been observed by many research with some variability. Christensen et al. [32], Burr et al. [35], and Lumley et al. [36] has found the solubility of $\mathrm{H}$ to be around 30-130 ppm, 50-60 ppm and $100 \mathrm{ppm}$, at reactor operating temperature respectively. The effect of temperature on the solubility of $\mathrm{H}$ is high, as Christensen et al. [32] have observed that solubility drops to $0.006 \mathrm{ppm}$ at $25^{\circ} \mathrm{C}$. Kumar [25] has found solubility levels of $6 \%, 0.70 \%$, and $0.10 \%$ respectively at $500^{\circ} \mathrm{C}, 300^{\circ} \mathrm{C}$, and room temperature. Blackmur et al. [10] states that the highest $\mathrm{H}$ solubility cannot exceed $3 \%$.

The solubility of $\mathrm{H}$ in $\mathrm{Zr}\left(S_{T}\right)$ has the form,

$$
S_{T}=S_{0} \exp \left(\frac{-E_{a}}{k_{B} T}\right)
$$

similar to the Arrhenius equation. It comprises of a temperature independent component $\left(S_{0}\right)$ and an exponent which represents the effect of temperature. Here, $k_{B}=8.3144 \mathrm{~mol}^{-1} \mathrm{~K}^{-1}$ is the Boltzmann constant, $T$ is the absolute temperature and $E_{a}$ is an energy similar to the activation energy found in reactions.

Constants $S_{0}$ and $k_{B}$ for TSSD (Total Solid Solubility in Dissolution) and TSSP (Total Solid Solubility in Precipitation) found by some researchers are presented in Table 1.2 below and Fig. 1.3 shows the variation in a graphical form for temperature 
Table 1.2: Coefficients for solubility of $\mathrm{H}$ in $\mathrm{Zr}$

\begin{tabular}{ccccc}
\hline \multirow{2}{*}{ Reference } & \multicolumn{2}{c}{ TSSD } & \multicolumn{2}{c}{ TSSP } \\
\cline { 2 - 5 } & $S_{0}(w t \mathrm{ppm})$ & $E_{a}(\mathrm{~J} / \mathrm{mol})$ & $S_{0}(w t \mathrm{ppm})$ & $E_{a}(\mathrm{~J} / \mathrm{mol})$ \\
\hline \hline Kearns [37] & $1.20 \times 10^{5}$ & 35900 & $3.15 \times 10^{4}$ & 27990 \\
Slattery [38] & $6.86 \times 10^{4}$ & 33570 & $4.11 \times 10^{4}$ & 28000 \\
Pan et al. [39] & $8.08 \times 10^{4}$ & 34520 & $2.47 \times 10^{4}$ & 25840 \\
Une \& Ishimoto [40] & $1.41 \times 10^{4}$ & $38104 \pm 701$ & $3.39 \times 10^{4}$ & $27291 \pm 553$ \\
\hline
\end{tabular}

Table 1.3: H solubility for reactor conditions

\begin{tabular}{ccccc}
\hline \multirow{2}{*}{ Reference } & \multicolumn{2}{c}{ TSSD } & \multicolumn{2}{c}{ TSSP } \\
\cline { 2 - 5 } & $\begin{array}{c}100^{\circ} \mathrm{C} \\
(\text { wtppm })\end{array}$ & $\begin{array}{c}300^{\circ} \mathrm{C} \\
(\text { wtppm })\end{array}$ & $\begin{array}{c}100^{\circ} \mathrm{C} \\
(\text { wtppm })\end{array}$ & $\begin{array}{c}300^{\circ} \mathrm{C} \\
(\text { wtppm })\end{array}$ \\
\hline \hline Kearns [37] & 1.127 & 64.059 & 3.790 & 88.466 \\
Slattery [38] & 1.365 & 59.720 & 4.929 & 115.185 \\
Pan et al. [39] & 1.184 & 57.625 & 5.945 & 108.931 \\
Une \& Ishimoto [40] & 0.650 & 47.382 & 5.110 & 110.251 \\
\hline
\end{tabular}

between 0 and $300^{\circ} \mathrm{C}$.

As seen in Fig. 1.3, relationships found from different studies only show minor variations within the considered temperature range. The variation for $\mathrm{H}$ mentioned above could be the difference between TSSD and TSSP. TSSP is the dissolved H concentration at which hydrides will start to precipitate and TSSD will show the dissolved $\mathrm{H}$ concentration at which the precipitated hydrides will start to dissolve. The precipitation will happen at a higher $\mathrm{H}$ concentration than the dissolution. Only the curves for TSSD from [40] and TSSP from [38] shows a noteworthy variation with the rest of the values. Solubility at extremes of most reactor temperatures $\left(100^{\circ} \mathrm{C}\right.$ and $300^{\circ} \mathrm{C}$ ) are shown in Table 1.3. The highest solubility for $\mathrm{H}$ under reactor conditions obtained is about $115 \mathrm{ppm}$ which is $1.03 \mathrm{~atm} \%$. These values are in agreement with the findings from other studies.

Of the sites available for occupancy for dissolved $\mathrm{H}$ in the $\mathrm{Zr}$ lattice, tetrahedral 


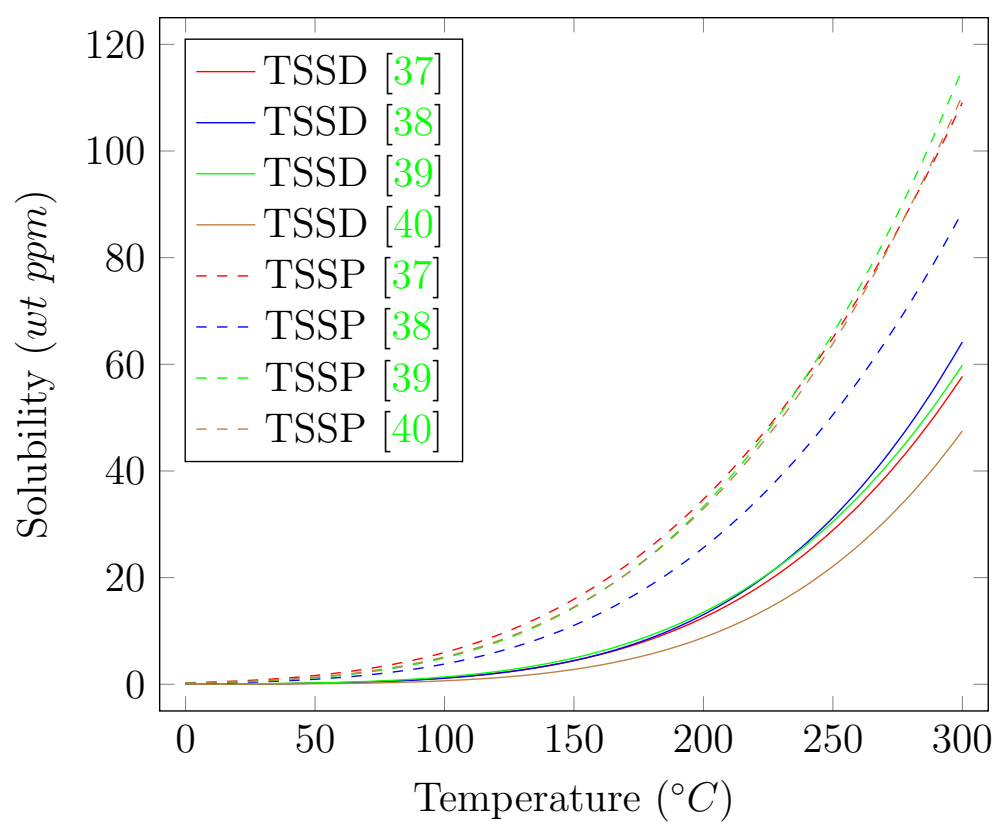

Figure 1.3: Variation of solubility of $\mathrm{H}$ in $\mathrm{Zr}$ with temperature

$\left(\mathrm{T}_{1}-\mathrm{T}_{4}\right.$ in Fig. 1.4) and octahedral interstitial sites $\left(\mathrm{O}_{1}\right.$ and $\mathrm{O}_{2}$ in Fig. 1.4) are the main locations $\mathrm{H}$ can occupy stably [35]. Of these two, $\mathrm{H}$ energetically prefer tetrahedral sites by a small margin. Comparing the binding energies of these two sites, Burr et al. [35] and Domain et al. [41] has found a preference for tetrahedral sites of $5.5 \mathrm{~kJ} \mathrm{~mol}^{-1}(0.057 \mathrm{eV})$ and $8.3 \mathrm{~kJ} \mathrm{~mol}^{-1}(0.086 \mathrm{eV})$ respectively. Christensen et al. [32] states that the variation of this preference could be due to the temperature, where at $0 \mathrm{~K}$ the preference is only about $0.5 \mathrm{~kJ} \mathrm{~mol}^{-1}(0.0052 \mathrm{eV})$ which increases to $8.6 \mathrm{~kJ} \mathrm{~mol}^{-1}(0.089 \mathrm{eV})$ at $600 \mathrm{~K}$. At $600 \mathrm{~K}$ only about $6 \%$ of the $\mathrm{H}$ is occupied in octahedral sites which will increase in lower temperatures.

\section{Zirconium Hydrides}

Once the solubility of $\mathrm{H}$ is exceeded, hydrides will start to form mainly in the grain boundaries as pellets or needle shaped precipitates [10] as shown in Fig. 1.5. There are three main types (phases) of zirconium hydride, of which two are stable and one is metastable [36]. The stable hydride phases are $\delta$ and $\varepsilon$ phases and the metastable phase is the $\gamma$-phase. As seen from the phase diagram in Fig. 1.6, the $\delta$-phase is predominantly observed at $\mathrm{H}$ concentrations below $H / Z r=1.6$. The metastable $\gamma$-phase has also been observed in the room temperature [36], where the cooling rate 


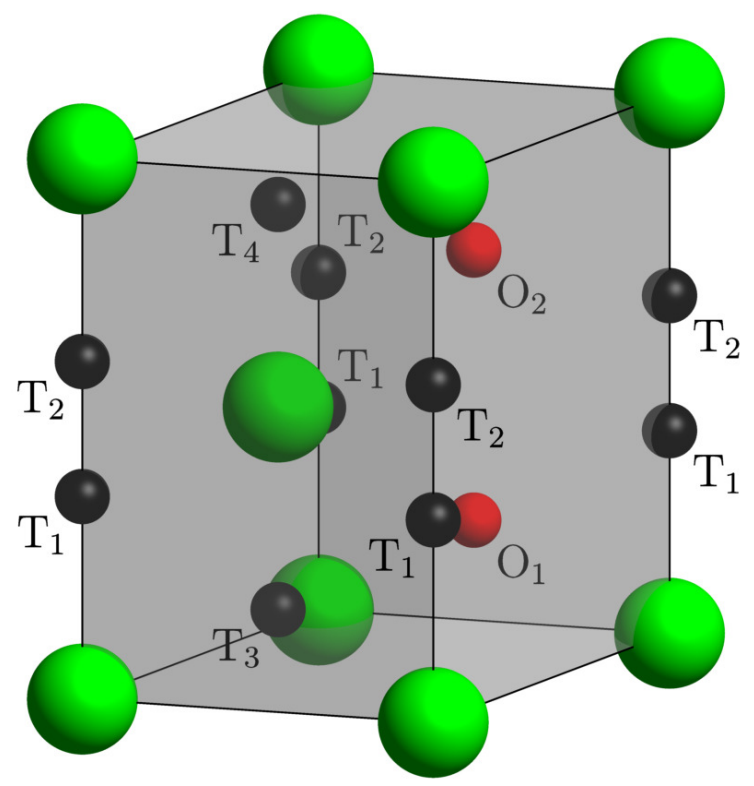

Figure 1.4: Interstitial sites of Zr lattice

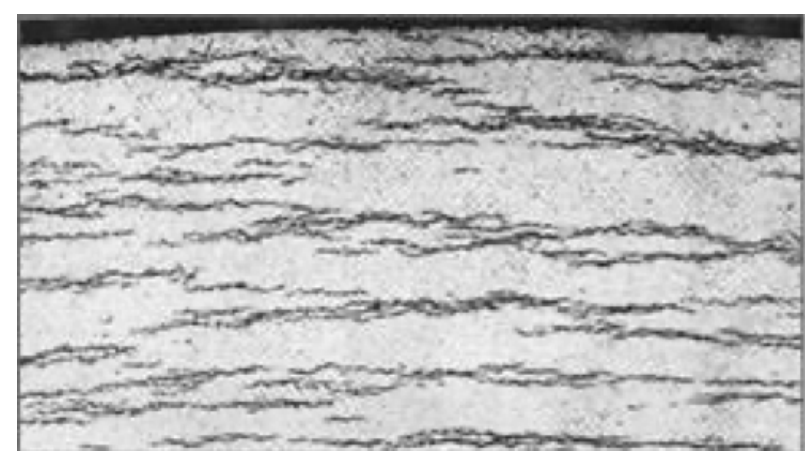

Figure 1.5: Microscopic view of a hydrided zircaloy tube [43]

is expected to be a main factor for the phase of hydride formed [42]. The $\delta$-phase is predominantly observed when cooling rate is below $2^{\circ} \mathrm{C} / \mathrm{min}$, while at faster cooling rates (greater than $10^{\circ} \mathrm{C} / \mathrm{min}$ ), the $\gamma$ phase is observed.

The $\delta$ phase has a face centered cubic (fcc) structure having a volume about $16 \%$ greater than that of $\alpha$-Zr lattice. This explains the preference of the hydrides to form in grain boundaries and the attraction of hydrides to regions under tensile strains [32]. The other two phases show face centered tetragonal (fct) structures. Table 1.4 shows some crystal details of the three hydride phases [13]. 


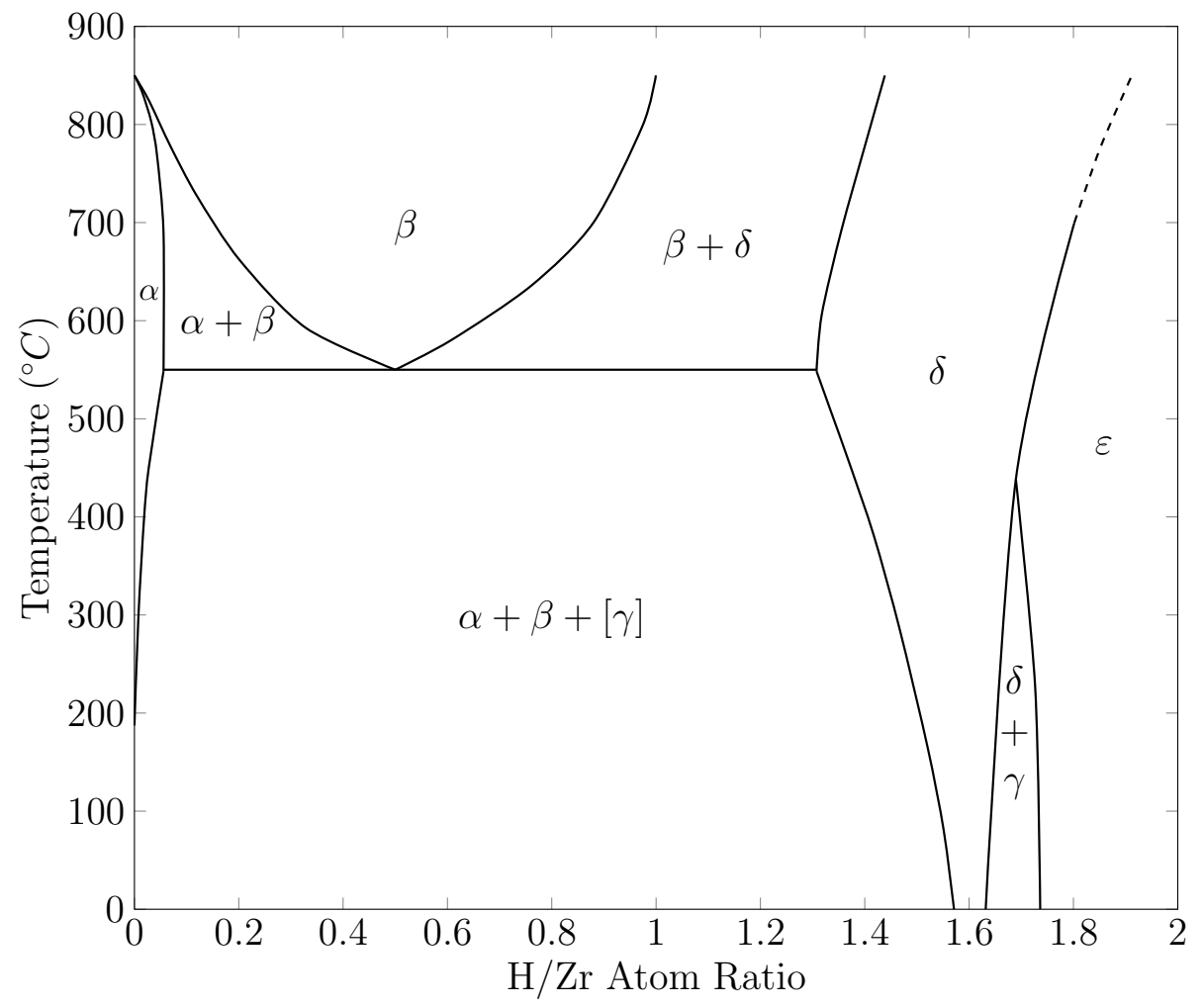

Figure 1.6: Phases of hydrogen in zirconium

Table 1.4: Crystal details of zirconium hydrides

\begin{tabular}{cccc}
\hline & $\delta$-phase & $\gamma$-phase & $\varepsilon$-phase \\
\hline \hline$a(\AA)$ & 4.775 & 4.592 & 4.999 \\
$c(\AA)$ & - & 4.492 & 4.433 \\
Space group & Cccm & Fm3m & I4/mmm \\
$H / Z r$ & $1.5-1.66$ & $1.75-2$ & 1 \\
\hline
\end{tabular}




\section{Hydrogen diffusion in zirconium}

Diffusion of particles is accepted to follow the Fick's first law [44], where diffusion flux is proportional to the concentration gradient as,

$$
J=-D \frac{\partial C}{\partial x}
$$

Where, $J$ is the diffusion flux and $D$ the proportional constant is called the diffusivity which can be used as a measure of the diffusion capacity. As per Arrhenius law [45],

$$
D=D_{0} \exp \left(\frac{-E_{a}}{k_{B} T}\right)
$$

the diffusion constant mainly depends on two parameters: $D_{0}$, the temperature independent component and $E_{a}$, the activation barrier for the diffusion which is contained in the temperature dependant exponent. More about diffusion theory is explained in Chapter 2.

Table 1.5 shows some of the diffusion coefficients available in literature for $\mathrm{H}$ diffusion in $\mathrm{Zr}$, obtained mainly by experimental means. These values show a very high variability. The activation energy, for the most part, shows a range of $0.45-$ $0.5 \mathrm{eV}$, and the temperature independent diffusion constant $D_{0}$ lies in the range $5-8 \times 10^{-3}$. In the expert opinion, values for $D_{0}$ obtained to within a factor of 5 is a good result. In the work of Kearns [46], the orientation of axes of Zr lattices in $\mathrm{Zr}$ pressure tubes were compared, where it was found that most of grains in the longitudinal direction were oriented in the C-axis (see Fig. 1.5) direction, while in the transverse direction, the grain orientation was mostly normal to the $\mathrm{C}$-axis direction. Thereby the author suggests that the diffusion of $\mathrm{H}$ in $\mathrm{Zr}$ is anisotropic with a higher diffusivity along the $\mathrm{C}$-axis direction, which can be expected due to the anisotropic crystal structure of Zr. But the radial direction has the lowest concentration of grains with $\mathrm{C}$-axis along the diffusion direction, which shows the highest diffusivity values. Kearns [46] also suggest that there is no significant effect of the alloying elements on diffusivity. Christensen et al. [32] holds the view that the diffusion of $\mathrm{H}$ in $\mathrm{Zr}$ is closely isotropic, a view which is also held by McRae et al. [47]. The latter authors propose that the reason for this variation is due to the hydrostatic stress and explains 
Table 1.5: Diffusion coefficients from literature for $\mathrm{H}$ in $\mathrm{Zr}$

\begin{tabular}{cccc}
\hline References & Details & $D_{0}\left(\mathrm{~cm}^{2} / \mathrm{s}\right)$ & $E_{a}(\mathrm{eV})$ \\
\hline \hline Christensen et al. [32] & Molecular Dynamics (KMC) & $1.13 \times 10^{-3}$ & - \\
& Longitudinal (experimental) & $7.73 \times 10^{-3}$ & 0.470 \\
Kearns [46] & Transverse (experimental) & $5.84 \times 10^{-3}$ & 0.446 \\
& Radial (experimental) & $7.90 \times 10^{-3}$ & 0.465 \\
Gulbransen \& Andrew [48] & H in Zr (experimental) & $1.09 \times 10^{-3}$ & 0.494 \\
Mallett \& Albrecht [49] & Deuterium in Zr (experimental) & $0.73 \times 10^{-3}$ & 0.494 \\
Someno [50] & Experimental & $7.00 \times 10^{-3}$ & 0.306 \\
Sawatsky [51] & $\alpha$-Zr (experimental 450 - 700 $\left.{ }^{\circ} \mathrm{C}\right)$ & $4.15 \times 10^{-3}$ & 0.411 \\
Mazzolai \& & $\alpha$-Zircaloy-2 (experimental) & $2.17 \times 10^{-3}$ & 0.363 \\
Ryll-Nardzewsi [52] & Experimental 870 - 1100 $\left.{ }^{\circ} \mathrm{C}\right)$ & $7.37 \times 10^{-3}$ & 0.370 \\
Naito [53] & $\alpha$-Zr (experimental) & $6.70 \times 10^{-3}$ & 0.475 \\
Siripurapu et al. [54] & Molecular Dynamics (EAM) & $1.69 \times 10^{-2}$ & 0.490 \\
Zhang et al. [55] & DFT \& KMC & $5.55 \times 10^{-3}$ & 0.410 \\
\hline
\end{tabular}

it with the extended version Fick's first law as:

$$
J=-D\left(\nabla C+\frac{C V}{k_{B} T} \nabla \sigma\right) .
$$

$V$ is the partial molar volume of hydrogen in zirconium and $\sigma$ is the hydrostatic stress. Another explanation presented is the existence of $\beta$ - $\mathrm{Zr}$ sheets embedded in the $\alpha$ - $\mathrm{Zr}$ lattice which acts as highways for $\mathrm{H}$ diffusion due to its higher diffusivity. This theory is not yet proven and also rejected by McRae et al. [47] who states that the $\mathrm{H}$ atoms will be lost in the larger $\alpha$-Zr grains and will have a low chance of meeting the $\beta$ - Zr sheets.

Muta et al. [56] have carried out an ab-initio study on the diffusion of $\mathrm{H}$ in monoclinic and tetragonal zirconium oxide. Where the diffusion coefficients $D_{0}$ and $E_{a}$ lies in ranges $3.10-12.5 \times 10^{-3} \mathrm{~cm}^{2} / \mathrm{s}$ and $1.244-2.166 \mathrm{eV}$ respectively. Even though $D_{0}$ shows a larger value, the activation barrier is much larger compared to the diffusion in $\mathrm{Zr}$ lattice which will give a much slower diffusivity value. At $200^{\circ} \mathrm{C}$ (selected to simulate reactor conditions), the diffusivity values from these studies are 
Table 1.6: Activation energies (in $\mathrm{eV}$ ) for transition steps for $\mathrm{H}$ diffusion in $\alpha$ - $\mathrm{Zr}$

\begin{tabular}{cccc}
\hline Transition Step & Domain et al. [41] & Zhang et al. [55] & Christensen et al. [57] \\
\hline \hline $\mathrm{T} \rightarrow$ NN T & 0.12 & 0.129 & 0.129 \\
$\mathrm{~T} \rightarrow$ NN O & 0.41 & 0.406 & 0.412 \\
$\mathrm{~T} \rightarrow$ 2nd NN T & 0.66 & - & - \\
$\mathrm{O} \rightarrow$ NN T & 0.35 & 0.346 & 0.360 \\
$\mathrm{O} \rightarrow$ NN O & 0.41 & 0.398 & 0.427 \\
\hline
\end{tabular}

in the range of $5.00 \times 10^{-26}$ to $1.74 \times 10^{-16} \mathrm{~cm}^{2} / \mathrm{s}$.

Diffusion of $\mathrm{H}$ will generally occur through an interstitial mechanism due to its smaller size. This requires a closer look at the various transition steps $\mathrm{H}$ takes. As seen in Fig. 1.4 the occupancy sites available for $\mathrm{H}$ are tetrahedral and octahedral sites. Hence, $\mathrm{H}$ diffusion is expected to consist of multiple transitions between the nearest tetrahedral or octahedral neighbor sites. Table 1.6 shows the activation energies obtained from some ab-inito calculations for these diffusion steps. Here T represents tetrahedral sites and $\mathrm{O}$ represents octahedral sites. These results will be used in our study to validate the models used in the current research.

\subsubsection{Oxygen in Zirconium}

Zirconium is used in nuclear and chemical plants due to its high corrosion resistance. But under harsh conditions (high temperature and pressure), it can form oxides with water, releasing $\mathrm{H}$ in radical and molecular gas form as shown in eqn. (1.1), even with the careful selection of materials [7]. LWRs need to operate at high temperatures to achieve its highest efficiencies, with PWRs needing to operate at $330^{\circ} \mathrm{C}$ and BWR needing to operate at $288^{\circ} \mathrm{C}$ [8], which is a favourable temperature for corrosion and $\mathrm{H}$ pickup in zirconium alloys. Most of the alloying elements in zirconium alloys are used to enhance corrosion resistance, such as niobium, tin, chromium, and iron.

The extent of corrosion resistance depends on characteristics such as [7]:

- Environmental conditions

- Construction process 
Table 1.7: Diffusion coefficients $\mathrm{O}$ in $\mathrm{Zr}$ available in literature

\begin{tabular}{cccc}
\hline References & Details & $D_{0}\left(\mathrm{~cm}^{2} / \mathrm{s}\right)$ & $E_{a}(\mathrm{eV})$ \\
\hline \hline Pemsler [58] & $400-585^{\circ} \mathrm{C}$ (experimental) & 9.4 & 2.203 \\
Quataert \& & $550-650^{\circ} \mathrm{C}$ (experimental) & $1.07 \times 10^{-1}$ & 1.973 \\
Coen-Porisini [59] & $650-850^{\circ} \mathrm{C}$ (experimental) & 15.0 & 2.372 \\
David at el. [60] & $700-845^{\circ} \mathrm{C}$ (experimental) & 1 & 2.125 \\
& $290-650^{\circ} \mathrm{C}$ & $6.62 \times 10^{-2}$ & 1.908 \\
Ritchie \& Atrens [61] & $($ review of literature) & & \\
& $650-1500^{\circ} \mathrm{C}$ & 16.5 & 2.372 \\
& $($ review of literature) & & \\
De González \& García [62] & Upto $230^{\circ} \mathrm{C}$ (experimental) & $3.1 \times 10^{-6}$ & 1.030 \\
\hline
\end{tabular}

- Material

- Mechanical load

The $\alpha$-phase of $\mathrm{Zr}$ (hcp) has a very high affinity to $\mathrm{O}$, with a very high solubility when compared with other solutes. In addition to oxidizing with water, oxygen in the atmosphere causes an oxygen gradient with the $\mathrm{Zr}$ lattice, causing $\mathrm{O}$ absorption. The solubility of $\mathrm{O}$ at $200^{\circ} \mathrm{C}$ is about $28.5 \mathrm{~atm} \%$ and it increases up to $35 \mathrm{~atm} \%$ at $2065^{\circ} \mathrm{C}$ [26]. Blackmur et al. [10] states that the general solubility of $\mathrm{O}$ in $\mathrm{Zr}$ is about $28.5 \mathrm{~atm} \%$. As a solute $\mathrm{O}$ prefers to occupy the octahedral site $\left(\mathrm{O}_{1}\right.$ and $\mathrm{O}_{2}$ in Fig. 1.4) as other locations will cause damage to the crystal structure of $\mathrm{Zr}$ making the lattice to rearrange locally [13]. The solubility levels are much higher than that of $\mathrm{H}$ in $\mathrm{Zr}$.

Table 1.7 shows some of the diffusion coefficients for $\mathrm{O}$ in diffusion in $\alpha$-Zr. The coefficients show a very high variation. The temperature dependency is noticeable with the $D_{0}$ which increases by several orders for temperatures above $650^{\circ} \mathrm{C}$. Comparing the diffusivity values between $\mathrm{O}$ and $\mathrm{H}$ at $200^{\circ} \mathrm{C}$, shows that $\mathrm{H}$ has a much higher diffusivity than $\mathrm{O}$, hence it is acceptable to consider that $\mathrm{O}$ to be stationary during the diffusivity studies carried out for hydrogen. 


\subsubsection{Hydride Denuded Zones}

This section contains a summary of the data received from Kinetrics [63] and Dr. Glenn McRae from Carleton University, who were instrumental in the initiation of the topic of this research. With the use of several studies it is expected to predict the thickness of the hydride denuded zones in zirconium pressure tubes so that scrape sampling can be modified to take samples which correctly represent the hydride content of the bulk.

The boundaries in the CANDU reactors consisting of $6 m$ long, tubes with a thickness of $4 \mathrm{~mm}$ and an internal diameter of $104 \mathrm{~mm}$, operate at a coolant pressure of around $10 \mathrm{MPa}$. Due to the susceptibility for failure from delayed hydride cracking (DHC), the pressure tubes with high hydride concentrations are replaced. The hydride concentrations are monitored through scrape samples taken from the tube surfaces which has usually proved to be representative of the hydride concentration in the bulk. It has been found that this is not the case for some older pressure tubes (after a few decades of operation) which contains a relatively high hydride concentration, where the surface has shown smaller and a fewer quantity of hydride precipitates than in the bulk.

Denuded zones have been observed in pressure tubes with a $\mathrm{H}$ concentration of 9-168 ppm having a thickness up to $193 \mu \mathrm{m}$. From previously available data no proper relationships can be identified for the denuded zone thickness to the $\mathrm{H}$ content or the exposure to irradiation. The denuded zone thickness has shown a weak relationship with the cooling rate, where at high cooling rates the observed denuded zones have decreases and at very high cooling rate $\left(10^{\circ} \mathrm{C} / \mathrm{min}\right)$ the denuded zone has not been observed (see Fig. 1.7). The oxygen concentrations near the surface and in the bulk is observed to be $424 \mathrm{~kg} / \mathrm{m}^{3}$ (65000 wt ppm 28.39\%) and $7.8 \mathrm{~kg} / \mathrm{m}^{3}$ (1200 wt ppm, $0.68 \%)$

There are three ideas that were put out as possible mechanisms for the formation of the hydride denuded zones.

- The pre-deposited hydride layer lowers the solubility of $\mathrm{H}$ closer to the surface which would cause a hydrogen gradient during cooling as the hydrogen in the bulk will get precipitated. This will cause the hydrogen near the surface to migrate to the bulk to compensate for the precipitated hydride creating a hydrogen shortage near the surface. The relationship shown for the denuded 


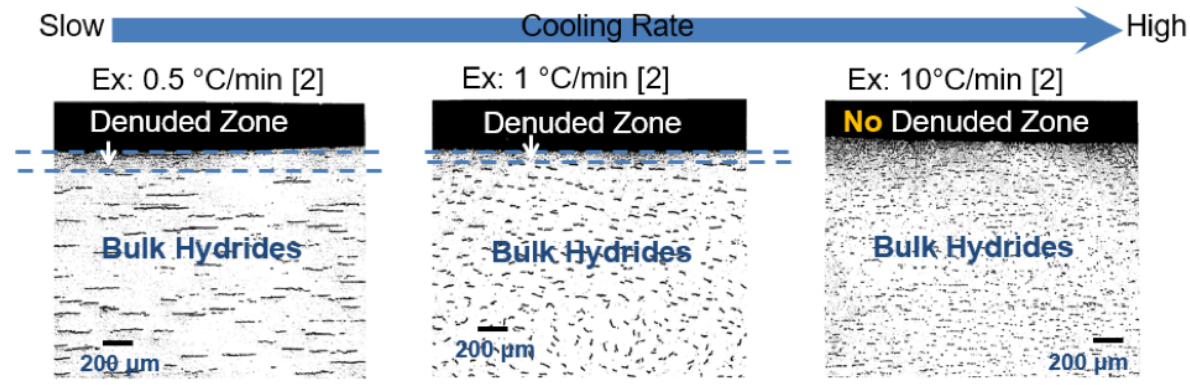

Figure 1.7: Effect of cooling rate on denuded zone [63]

zone with the cooling rate has been used as an argument for this idea, where at higher cooling rates, the $\mathrm{H}$ may not have enough time to diffuse to the bulk, which will result in hydrides even near the surface. A counter argument exists to this mechanism, which suggests that hydrogen in the pre-deposited hydrides will flow into the denuded zone which then can be precipitated.

- The second mechanism proposed is that the oxide layer in the surface will cause a tensile stress at the oxide-metal interface, attracting hydrogen closest to it and depleting the hydrogen in the near-surface region. Similar to the above mechanism the counter argument is that hydrogen in the bulk will flow due to the formed hydrogen gradient.

- The third mechanism as Hardie [15] proposed is an explanation to the presence of hydride depleted zones, with oxygen affecting the diffusion of hydrogen via blocking of interstitial sites and slowing down of hydrogen, making it harder to accumulate to form large precipitates in the grain boundaries. Instead the precipitates formed will be fine and intragranular.

Looking at these hypothesis we wanted to check how interstitial $\mathrm{O}$ and hydrostatic stresses affect the diffusivity of $\mathrm{H}$ in $\mathrm{Zr}$ lattice. Using a multi-scale model we were able to identify these effects. Thereafter, we used the identified effects of of interstitial O in a $\mathrm{O}$ concentration profile, estimated for reactor conditions, to check the validity of the third hypothesis above. We expect to use the identified effect in diffusivity with respect to hydrostatic stress to check how the concentration profiles of $\mathrm{H}$ is affected when it is under the operating conditions of a reactor. 


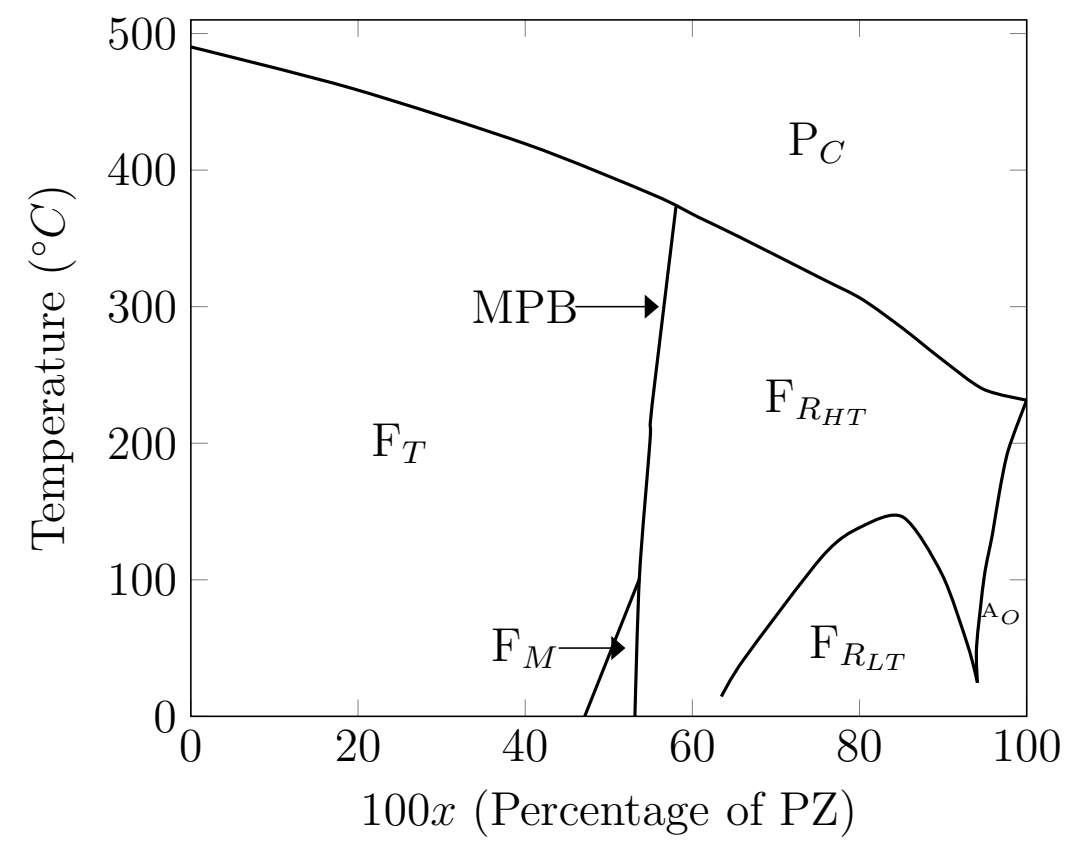

Figure 1.8: Phase diagram of $\mathrm{PZT}$ with respect to temperature and $\mathrm{PZ}: \mathrm{PT}$ ratio

\subsubsection{Lead Zirconate Titanate}

Lead zirconate titanate $\left(\mathrm{PbZr}_{x} \operatorname{Ti}_{(1-x)} \mathrm{O}_{3}, \mathrm{PZT}\right)$ is a combination of two weak piezoelectric material lead zirconate $\left(\mathrm{PbZrO}_{3}, \mathrm{PZ}\right)$ and lead titanate $\left(\mathrm{PbTiO}_{3}, \mathrm{PT}\right)$ which has superior dielectric and piezoelectric properties [64]. First synthesized in 1952 [65], PZT is it made up of varying combinations of PZ and PT to have different crystal structures and different piezoelectric properties. The phase diagram for PZT based on the ratio of PZ:PT and temperature is as shown in Fig. 1.8, where the transition between the tetragonal structure $\left(\mathrm{F}_{T}\right)$ and rhombohedral structure $\left(\mathrm{F}_{R_{H T}}\right.$ and $\mathrm{F}_{R_{L T}}$ ) happen at morphological phase boundary (MPB). Close to this (where $x \approx 0.5-0.55[20,65,66])$ PZT shows the best piezoelectric properties, which is due to a monoclinic phase $\left(\mathrm{F}_{M}\right)$ happening at this combination [66]. While the crystal properties of this structure $\left(\mathrm{F}_{M}\right)$ is not fully established, we considered $x=0.5$ and a tetragonal crystal structure (see Fig. 1.9) for our research purposes.

A thorough literature review is included in Chapter 6, discussing PZT structure, hydrogen degradation of PZT, and H diffusion in PZT.

In this study we took the initial steps at estimating the diffusivity of $\mathrm{H}$ in the 


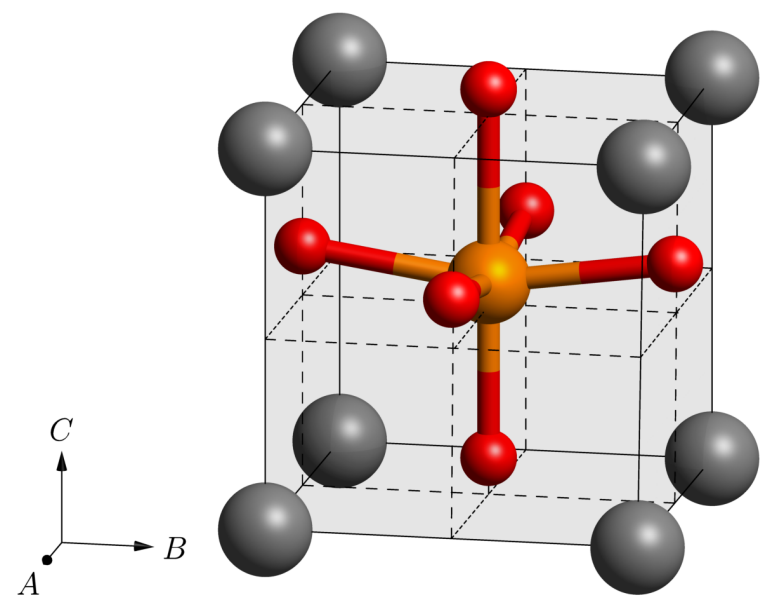

Figure 1.9: Tetragonal crystal structure of PZT, where grey spheres represent $\mathrm{Pb}$ atoms, red spheres represent $\mathrm{O}$ atoms and orange sphere represent either $\mathrm{Zr}$ or $\mathrm{Ti}$ atoms occurring in a random order

PZT lattice using the diffusion model we validated using Zr. We identified the locations available for $\mathrm{H}$ occupancy in the PZT lattice using first principles calculations. Thereafter we predicted the possible transition paths available for $\mathrm{H}$ to transition between these sites. Future direction is to carry out simulations to find the transition rates between these sites, which can be used as input in KMC simulations. Using KMC simulations the diffusivity of $\mathrm{H}$ in PZT can be estimated.

\subsection{Scope and Objectives}

The primary motive of our research is to investigate two of the hypotheses which are proposing mechanisms for the formation of hydride denuded zones. With the use of multi-scale models, we wanted to look at the effect interstitial $\mathrm{O}$ and hydrostatic stress has on diffusivity of $\mathrm{H}$ in $\mathrm{Zr}$, to identify any evidence available to prove or disprove these hypotheses. Then we wanted to further extend our study to investigate $\mathrm{H}$ diffusion in PZT, a piezoelectric material which is also adversely affected by $\mathrm{H}$ absorption. As the initial step we did an investigation of $\mathrm{H}$ behavior in PZT to identify the stable $\mathrm{H}$ occupancy locations and the effect these interstitial $\mathrm{H}$ will have on the performance of PZT. Based on the above we defined the following objectives for this research.

1. Develop a multi-scale model to simulate the diffusion behavior of $\mathrm{H}$, when the 
occupancy locations and the transition steps are known.

2. Verify the developed model using the literature available on $\mathrm{H}$ diffusion in Zr.

3. Identify the transition pathways available for interstitial diffusion of $\mathrm{H}$, with varied effects from neighbouring $\mathrm{O}$ in $\mathrm{Zr}$ lattice, and obtain the energy landscape for the identified diffusion steps and find the relevant diffusion coefficients.

4. Using the validated model simulate the diffusion of $\mathrm{H}$ in $\mathrm{Zr}$ for various $\mathrm{O}$ concentrations to predict the diffusivity of $\mathrm{H}$ for each different concentration.

5. Quantify the effect of $\mathrm{O}$ on the $\mathrm{H}$ diffusivity and investigate the reasons for the variation in diffusivity.

6. Investigate the validity of the hypothesis, which suggests that $O$ near the surface of pressure tubes causes denuded zones.

7. Extend the diffusion model to determine the effect of stress on $H$ diffusivity through the same procedure.

8. Investigate the occupancy locations available for $\mathrm{H}$ in the PZT crystal structure and identify the transition pathways between these sites.

9. Investigate the effect on the polarization properties of PZT due to the presence of $\mathrm{H}$.

\subsection{Publications}

The following journal papers and conference presentations related to the thesis were produced during the course of this study. The main body of the thesis is made from three complete papers and most of a fourth paper which is to be submitted soon.

\subsubsection{Journal Papers}

- Manura Liyanage, Ronald Miller, and R. K. N. D. Rajapakse, "Multiscale approach for determining hydrogen diffusivity in zirconium", Modelling and Simulation in Materials Science and Engineering, vol. 26, no. 8, pp. 085002 1-20, 2018. 
- Manura Liyanage, Ronald Miller, and R. K. N. D. Rajapakse, "Firstprinciples study of hydrogen in lead zirconate titanate", Smart Materials and Structures, vol. 28, no. 3, pp. 034002 1-11, 2019.

- Manura Liyanage, Ronald Miller, and R. K. N. D. Rajapakse, "Denuded Zones in Zirconium Pressure Vessels: Oxygen's Role Examined via Multi-scale Diffusion Model", under review in Modelling and Simulation in Materials Science and Engineering, (Submission: January 2020).

- Manura Liyanage, Ronald Miller, and R. K. N. D. Rajapakse, "Effect of Stress on Hydrogen Diffusion in Zirconium, investigated through multi-scale methods", most work related to this publication is completed and expected to be submitted soon.

\subsubsection{Conference presentations}

- Manura Liyanage, Ronald Miller, and R. K. N. D. Rajapakse, "Effects of Oxygen on Hydrogen Diffusion in Zirconium", 29th Canadian Material Science Conference, Ottawa, ON, June 2017.

- Manura Liyanage, Ronald Miller, and R. K. N. D. Rajapakse, "Firstprinciples study of hydrogen in lead zirconate titanate", IUTAM Symposium on Mechanics of Electro/Magneto-Active Materials and Structures, Beijing, China, August 2018.

\subsection{Organization of the Thesis}

Chapter 1 of the thesis introduces the research problem and provides an extensive literature review on many areas related to the problems under investigation. The next chapter (Chapter 2) consists of two sections. Sec. 2.1 presents the diffusion theories adopted in the research to come up with the diffusion model and its improvements. Sec. 2.2 presents the details of the computational methods used in the thesis. The main body of the thesis follows next, which is made up of three complete papers (Chapter 3, Chapter 4, and Chapter 6), and most of a fourth paper (Chapter 5) which is being completed at the time of thesis submission. Each of these chapters has 
its own introduction, content and conclusions. The order of the chapters are different from the order of work carried out to help with the flow of the thesis.

Chapter 3 presents the initial multi-scale model we used to simulate the diffusion of $\mathrm{H}$ in pure $\mathrm{Zr}$. This chapter validates the model we are using in the remaining sections to investigate different problems related to $\mathrm{H}$ diffusion. Here we also investigate the anisotropy of $\mathrm{H}$ diffusion in $\mathrm{Zr}$, which shows contradicting results in literature. In Chapter 4, we look at how the diffusivity is affected by the presence of $\mathrm{O}$ in $\mathrm{Zr}$. We used the model verified in Chapter 3 with some improvements. Here the change in diffusivity is quantified with the changing $\mathrm{O}$ concentration and the results are mapped against the $\mathrm{O}$ concentration profiles expected in $\mathrm{Zr}$ pressure tubes. With this we checked the validity of the hypothesis suggesting that denuded zones in $\mathrm{Zr}$ pressure tubes occur due to the presence of interstitial O in Zr PTs. Chapter 5 utilizes the improved diffusion model in Chapter 4 to simulate $\mathrm{H}$ diffusion under different hydrostatic stresses to quantify their effect. The penultimate chapter, (Chapter 6) contains the initial work carried out on PZT, another material which is affected by $\mathrm{H}$ absorption. We identify the $\mathrm{H}$ occupancy locations in the PZT structure and proposes transition pathways available for $\mathrm{H}$ diffusion in the PZT lattice. We also check the effect $\mathrm{H}$ has on the polarization of PZT which is identified as a main problem in PZT due to $\mathrm{H}$ absorption. Finally we present the conclusions in Chapter 7.

\section{Bibliography}

[1] World Nuclear Association, "Nuclear Power in the World Today." https://www. world-nuclear.org/information-library/ current-and-future-generation/nuclear-power-in-the-world-today . aspx. Accessed: 2020 Feb. 29.

[2] U.S. Energy Information Administration, "Nuclear explained - Nuclear power plants." https://www.eia.gov/energyexplained/nuclear/ nuclear-power-plants. php. Accessed: 2020 Feb. 29.

[3] S. Schlömer, T. Bruckner, L. Fulton, E. Hertwich, A. McKinnon, D. Perczyk, J. Roy, R. Schaeffer, R. S. amd P. Smith, and R. Wiser, "Annex iii: Technologyspecific cost and performance parameters.," in Climate Change 2014: Mitigation of Climate Change. Contribution of Working Group III to the Fifth Assessment Report of the Intergovernmental Panel on Climate Chang (O. Edenhofer, R. Pichs-Madruga, Y. Sokona, E. Farahani, S. Kadner, K. Seyboth, A. Adler, I. Baum, S. Brunner, P. Eickemeier, B. Kriemann, J. Savolainen, S. Schlömer, C. von Stechow, T. Zwickel, and J. C. Minx, eds.), p. 1329-1356, Cambridge, United Kingdom and New York, NY, USA: Cambridge University Press, 2014. 
[4] A. Markandya and P. Wilkinson, "Electricity generation and health," Lancet, vol. 370, pp. 979-990, 2007.

[5] Natural Resources Canada, "Uranium and nuclear power facts." https:// www.nrcan.gc.ca/science-data/data-analysis/energy-data-analysis/ energy-facts/uranium-and-nuclear-power-facts/20070\#L1. Accessed: 2020 Feb. 29.

[6] J. Parson, "Nasa plans to put nuclear reactors on mars to power a human colony - mirror online." https://www.mirror.co.uk/science/ nasa-plans-put-nuclear-reactors-10729812. Accessed: 2020 Feb. 17.

[7] E. Chajduk and A. Bojanowska-Czajka, "Corrosion mitigation in coolant systems in nuclear power plants," Progress in Nuclear Energy, vol. 88, pp. 1-9, 2016.

[8] A. T. Motta, A. Couet, and R. J. Comstock, "Corrosion of zirconium alloys used for nuclear fuel cladding," Annual Review of Materials Research, vol. 45, p. 311-343, 2015.

[9] International Atomic Energy Agency, Corrosion of zirconium alloys in nuclear power plants. Vienna, Austria: International Atomic Energy Agency, 1993.

[10] M. S. Blackmur, J. D. Robson, M. Preuss, O. Zanellato, R. J. Cernik, S. Q. Shi, F. Ribeiro, and J. Andrieux, "Zirconium hydride precipitation kinetics in zircaloy-4 observed with synchrotron x-ray diffraction," Journal of Nuclear Materials, vol. 464, p. 160-169, 2015.

[11] R. Tapping, "Corrosion issues in pressurized heavy water reactor (PHWR/CANDUR) systems," in Nuclear Corrosion Science and Engineering (D. Féron, ed.), Woodhead Publishing Series in Energy, pp. 581 - 633, Woodhead Publishing, 2012.

[12] R. Krishnan and M. K. Asundi, "Zirconium alloys in nuclear technology," in Proceedings of the Indian Academy of Sciences Section C: Engineering Sciences, vol. 4, pp. 41-56, 1981.

[13] M. V. Glazoff, "Modeling of some physical properties of zirconium alloys for nuclear applications in support of ufd campaign," tech. rep., Idaho National Laboratory, UFD Campaign, Idaho Falls, Idaho, Aug 2013.

[14] S. Suman, M. K. Khan, M. Pathak, R. N. Singh, and J. K. Chakravartty, "Hydrogen in Zircaloy: Mechanism and its impacts," International Journal of Hydrogen Energy, vol. 40, pp. 5976-5994, 2015.

[15] D. Hardie, "The effect of oxygen on the precipitation of hydrogen from zirconium," Journal of Nuclear Materials, vol. 17, pp. 88-90, 1965.

[16] E. Polatidis, P. Frankel, J. Wei, M. Klaus, R. Comstock, A. Ambard, S. Lyon, R. Cottis, and M. Preuss, "Residual stresses and tetragonal phase fraction characterisation of corrosion tested zircaloy-4 using energy dispersive synchrotron x-ray diffraction," Journal of Nuclear Materials, vol. 432, no. 1, pp. 102 - 112, 2013. 
[17] P. Platt, E. Polatidis, P. Frankel, M. Klaus, M. Gass, R. Howells, and M. Preuss, "A study into stress relaxation in oxides formed on zirconium alloys," Journal of Nuclear Materials, vol. 456, pp. 415 - 425, 2015.

[18] Y.-S. Kim, Y.-H. Jeong, and J.-N. Jang, "Stress measurements during thin film zirconium oxide growth," Journal of Nuclear Materials, vol. 412, pp. 217-220, 2011.

[19] R. A. Ribeiro and S. R. de Lazaro, "DFT/PBE0 study on structural, electronic and dielectric properties of $\mathrm{SnZr}_{0.50} \mathrm{Ti}_{0.50} \mathrm{O}_{3}$ lead-free ferroelectric material," $J$. Alloy. Compd., vol. 714, pp. 553-559, 2017.

[20] T. Kitamura, Y. Umeno, F. Shang, T. Shimada, and K. Wakahara, "Development of Interatomic Potential for $\mathrm{Pb}(\mathrm{Zr}, \mathrm{Ti}) \mathrm{O}_{3}$ Based on Shell model," J. Solid Mech. Mater. Eng., vol. 1, no. 12, pp. 1423-1431, 2007.

[21] D.-J. Shin, S.-J. Jeong, C.-E. Seo, K.-H. Cho, and J.-H. Koh, "Multi-layered piezoelectric energy harvesters based on PZT ceramic actuators," Ceram. Int., vol. 41, pp. S686-S690, 2015. 9th Asian Meeting on Electroceramics (AMEC-9).

[22] Y. Singh, R. K. N. D. Rajapakse, E. Kjeang, and D. Mumford, "Performance of piezoelectric actuators in a hydrogen environment: Experimental study and finite element modelling," Int. J.Hydrogen Energ., vol. 40, no. 8, pp. 3370-3380, 2015 .

[23] F. Willaime, "Ab initio study of self-interstitials in hcp-Zr," Journal of Nuclear Materials, vol. 323, pp. 205-212, 2003.

[24] J. L. Zarestky, Lattice dynamics of hcp and bcc zirconium. PhD thesis, Iowa State University, 1979.

[25] N. A. P. K. Kumar, Hydride formation in zirconium alloys. PhD thesis, McGill University, 2011.

[26] C. Anghel, Modified oxygen and hydrogen transport in Zr-based oxides. PhD thesis, Royal Institute of Technology, KTH, 2006.

[27] J. Song and W. A. Curtin, "A nanoscale mechanism of hydrogen embrittlement in metals," Acta Materialia, vol. 59, p. 1557-1569, 2011.

[28] M. L. Martin, B. P. Somerday, R. O. Ritchie, P. Sofronis, and I. M. Robertson, "Hydrogen-induced intergranular failure in nickel revisited," Acta Materialia, vol. 60, p. 2739-2745, 2012.

[29] A. Turnbull, "Perspectives on hydrogen uptake, diffusion and trapping," International Journal of Hydrogen Energy, vol. 40, p. 16961-16970, 2015.

[30] G. Lu and E. Kaxiras, "Hydrogen embrittlement of aluminum: The crucial role of vacancies," Physical Review Letters, vol. 94, no. 15, pp. 155501 1-5, 2005.

[31] J. Song and W. A. Curtin, "Atomic mechanism and prediction of hydrogen embrittlement in iron.," Nature materials, vol. 12, no. 2, pp. 145-151, 2013. 
[32] M. Christensen, W. Wolf, C. Freeman, E. Wimmer, R. B. Adamson, L. Hallstadius, P. Cantonwine, and E. V. Mader, "Effect of hydrogen on dimensional changes of zirconium and the influence of alloying elements: First-principles and classical simulations of point defects, dislocation loops, and hydrides," in STP1543-EB Zirconium in the Nuclear Industry (B. Comstock and P. Barberis, eds.), vol. 17, pp. 55-92, West Conshohocken, PA: ASTM International, 2015.

[33] M. Ma, W. Xiang, B. Tang, L. Liang, L. Wang, and X. Tan, "Non-isothermal and isothermal hydrogen desorption kinetics of zirconium hydride," Journal of Nuclear Materials, vol. 467, pp. 349-356, 2015.

[34] A. E. Evsin, L. B. Begrambekov, A. I. Gumarov, A. S. Kaplevsky, A. G. Luchkin, L. R. Tagirov, and I. R. Vakhitov, "Trapping and desorption of hydrogen isotopes under irradiation of zirconium by deuterium atoms of thermal energies," Vacuum, vol. 129, p. 183-187, 2016.

[35] P. A. Burr, S. T. Murphy, S. C. Lumley, M. R. Wenman, and R. W. Grimes, "Hydrogen accommodation in zr second phase particles: implications for h pickup and hydriding of zircaloy-2 and zircaloy-4," Corrosion Science, vol. 69, pp. 1$4,2013$.

[36] S. C. Lumley, R. W. Grimes, S. T. Murphy, P. A. Burr, A. Chroneos, P. R. ChardTuckey, and M. R. Wenman, "The thermodynamics of hydride precipitation: The importance of entropy, enthalpy and disorder," Acta Materialia, vol. 79, pp. 351362,2014 .

[37] J. J. Kearns, "Terminal solubility and partitioning of hydrogen in the alpha phase of zirconium, Zircaloy-2 and Zircaloy-4," Journal of Nucelar Materials, vol. 22, p. 292-303, 1967.

[38] G. F. Slattery, "The terminal solubility of hydrogen in zirconium alloys between $30^{\circ} \mathrm{C}$ and $400^{\circ} \mathrm{C}$," Journal of the Institute of Metals, vol. 95, 1967.

[39] Z. L. Pan, I. G. Ritchie, and M. P. Puls, "The terminal solid solubility of hydrogen and deuterium in Zr-2.5Nb alloys," Journal of Nucelar Materials, vol. 228, p. 227-237, 1996.

[40] K. Une and S. Ishimoto, "Terminal Solid Solubility of Hydrogen in Unalloyed Zirconium by Differential Scanning Calorimetry," Journal of Nuclear Science and Technology, vol. 41, p. 949-952, 2004.

[41] C. Domain, R. Besson, and A. Legris, "Atomic-scale ab-initio study of the zrh system: I. bulk properties," Acta Materialia, vol. 50, no. 13, pp. 3513-3526, 2002 .

[42] S. D. Kim, Y. Rhyim, J. S. Kim, and J. Yoon, "Characterization of zirconium hydrides in Zircaloy-4 cladding with respect to cooling rate," Journal of Nucelar Materials, vol. 465, p. 731-736, 2015.

[43] Capital Energy, "Hydriding." http://capitalenergy.biz/?p=25905. Accessed: 2017 Jul. 04. 
[44] A. Fick, "Ueber diffusion," Annalen der Physik, vol. 170, pp. 59-86, 1855.

[45] S. Arrhenius, "Über die dissociationswärme und den einfluss der temperatur auf den dissociationsgrad der elektrolyte," Zeitschrift für Physikalische Chemie, vol. $4 \mathrm{U}$, pp. $96-116,1889$.

[46] J. J. Kearns, "Diffusion coefficient of hydrogen in alpha zirconium, zircaloy-2 and zircaloy-4," Journal of Nuclear Materials, vol. 43, no. 3, pp. 330-338, 1972.

[47] G. McRae, C. Coleman, H. Nordin, B. Leitch, and S. Hanlon, "Diffusivity of hydrogen isotopes in the alpha phase of zirconium alloys interpreted with the Einstein flux equation," Journal of Nuclear Materials, vol. 510, pp. 337-347, 2018.

[48] E. A. Gulbransen and K. F. Andrew, "Diffusion of hydrogen and deuteriun in high purity zirconium," Journal of the Electrochemical Society, vol. 101, no. 11, pp. 560-566, 1953.

[49] M. W. Mallett and W. M. Albrecht, "Low-pressure solubility and diffusion of hydrogen in zirconium," Journal of The Electrochemical Society, vol. 104, no. 3, pp. 142-146, 1957.

[50] M. Someno, "Solubility and diffusion of hydrogen in zirconium," Nippon Kinzoku Gakkaishi (Japan), vol. 24, pp. 249-253, 1960.

[51] A. Sawatzky, "The diffusion and solubility of hydrogen in the alpha phase of zircaloy-2," Journal of Nuclear Materials, vol. 2, no. 1, pp. 62-68, 1960.

[52] F. Mazzolai and J. Ryll-Nardzewsi, "An anelastic study of the diffusion coefficient of hydrogen in $\alpha$-zirconium," Journal of the Less-Common Metalls, vol. 49, pp. 323-327, 1976.

[53] S. Naito, "Kinetics of hydrogen absorption by $\alpha$-zirconium," The Journal of Chemical Physics, vol. 79, no. 6, pp. 3113-3120, 1983.

[54] R. K. Siripurapu, B. Szpunar, and J. A. Szpunar, "Molecular Dynamics Study of Hydrogen in $\alpha$-Zirconium," International Journal of Nuclear Energy, vol. 2014, 2014.

[55] Y. Zhang, C. Jiang, and X. Bai, "Anisotropic hydrogen diffusion in $\alpha$-Zr and Zircaloy predicted by accelerated kinetic Monte Carlo simulations," Scientific Reports, vol. 7, pp. 41033 1-13, 2017.

[56] H. Muta, Y. Etoh, Y. Ohishi, K. Kurosaki, and S. Yamanaka, "Ab initio study of hydrogen diffusion in zirconium oxide," Journal of Nuclear Science and Technology, vol. 49, no. 5, pp. 544-550, 2012.

[57] M. Christensen, W. Wolf, C. Freeman, E. Wimmer, R. B. Adamson, L. Hallstadius, P. E. Cantonwine, and E. V. Mader, "H in $\alpha$-zr and in zirconium hydrides: solubility, effect on dimensional changes, and the role of defects," Journal of Physics: Condensed Matter, vol. 27, pp. 025402 1-12, 2015. 
[58] J. P. Pemsler, "Diffusion of oxygen in zirconium and its relation to oxidation and corrosion," Journal of the Electrochemical Society, vol. 105, pp. 315-322, 1958.

[59] D. Quataert and F. Coen-Porisini, "Utilization of the ion analyser for the study of oxygen diffusion in solids and its application to zirconium," Journal of Nuclear Materials, vol. 36, pp. 20-28, 1970.

[60] D. David, G. Amsel, P. Boisot, and G. Beranger, "A study of the diffusion of oxygen in alpha-zirconium by means of nuclear microanalysis," Journal of the Electrochemical Society, vol. 122, pp. 388-396, 1975.

[61] I. G. Ritchie and A. Atrens, "The diffusion of oxygen in alpha-zirconium," Journal of Nuclear Materials, vol. 67, p. 254-264, 1977.

[62] C. O. D. González and E. A. García, "Determination of the diffusion coefficients of oxygen in zirconium by means of xps," Applied Surface Science, vol. 44, pp. 211-219, 1990.

[63] J. Hsu, J. Cui, A. Celovsky, E. Tulk, and Kinetrics, "Prediction of hydrogen isotope denuded zone in pressure tube," 2016. Private presentation.

[64] I. Grinberg, V. R. Cooper, and A. M. Rappe, "Oxide chemistry and local structure of $\mathrm{PbZr}_{x} \mathrm{Ti}_{1-x} \mathrm{O}_{3}$ studied by density-functional theory supercell calculations," Phys. Rev. B, vol. 69, no. 14, p. 144118, 2004.

[65] A. Gopejenko, S. Piskunov, and Y. F. Zhukovskii, "Ab initio modelling of the effects of varying $\mathrm{Zr}(\mathrm{Ti})$ concentrations on the atomic and electronic properties of stoichiometric PZT solid solutions," Comput. Theor. Chem., vol. 1104, pp. 5660, 2017.

[66] B. Noheda, D. E. Cox, G. Shirane, L. E. Cross, and S.-E. Park, "A monoclinic ferroelectric phase in the $\mathrm{Pb}\left(\mathrm{Zr}_{1-x} \mathrm{Ti}_{x}\right) \mathrm{O}_{3}$ solid solution," Appl. Phys. Lett., vol. 74, no. 14 , pp. 2059-2061, 1999. 


\section{Chapter 2}

\section{Diffusion Theory and Computational Methods}

In this chapter we present the diffusion theories and the computational methods used in this thesis. Sec. 2.1 which is on on diffusion theories looks at Fick's laws, random walk theory, and transition state theories (and derivation of some of them), which we used to develop the multi-scale model to simulate $\mathrm{H}$ diffusion in lattice structures. Once the model is developed we use first principles calculations to look at the atomic level diffusion parameters which feeds as input data for the bulk simulation models. Details about the computational methods used are presented in Sec. 2.2.

\subsection{Diffusion of Solids}

Diffusion studies were first experimentally carried out by Thomas Graham who was a professor in chemistry at Andersonian Institute (Now University of Strathclyde in Glasgow). His experiments for diffusion in salt-water systems instigated the interest of Adolf Fick on diffusion, which led to the creation of Fick's laws. The most vital contribution of Fick is the definition of the diffusion coefficient (or diffusivity). Svante August Arrhenius developed the Arrhenius law which identifies the temperature dependence of diffusion. Brownian motion was first discovered by Robert Brown through observations of the movement of pollen in water. Einstein and Smoluchowski came up with a theory to explain Brownian motion. These are some of the major milestones in the development of diffusion science. This chapter contains some of the diffusion related theories and models which are applied in the our study. 


\subsubsection{Fick's Law}

Fick's laws are the fundamental relationships accepted for diffusion by the academic community. This section briefly discussed useful relationships from Fick's laws as adopted from Mehrer [1]. For one-dimensional diffusion ( $X$-direction), the Fick's law states that the diffusion flux is proportional to the concentration gradient,

$$
J=-D \frac{\partial C}{\partial x},
$$

where $J$ is the diffusion flux and $C$ is the concentration at $x$. The negative sign shows that the diffusion occurs from high to low concentration locations. The proportional constant $D$ is called the diffusivity which has units of length ${ }^{2}$ per unit time.

This can be extended to isotropic diffusion in three-dimension as,

$$
\mathbf{J}=-D \nabla C=-D\left(\mathbf{i} \frac{\partial C}{\partial x}+\mathbf{j} \frac{\partial C}{\partial y}+\mathbf{k} \frac{\partial C}{\partial z}\right)
$$

In an anisotropic medium, the diffusivity becomes a second order tensor. The diffusion equation changes to,

$$
\mathbf{J}=-\mathbf{D} \nabla C
$$

If the principle diffusion directions were taken, the diffusion tensor will contain three independent terms,

$$
\mathbf{D}=\left(\begin{array}{ccc}
D_{1} & 0 & 0 \\
0 & D_{2} & 0 \\
0 & 0 & D_{3}
\end{array}\right)
$$

For hcp crystals as found in $\alpha-\mathrm{Zr}$, the diffusion along the basal plane is consistent, meaning:

$$
D_{1}=D_{2} \neq D_{3},
$$

where $D_{3}$ is the crystal axis direction.

Fick's first law can be combined with the continuity equation to come up with the Fick's second law, which is also referred to as the diffusion equation. This is a second order partial differential equation which combines the concentration gradient to the variation of concentration through time. Continuity of diffusion can be derived by looking at particle inflow and outflow through a small volume as shown in Fig. 2.1. 


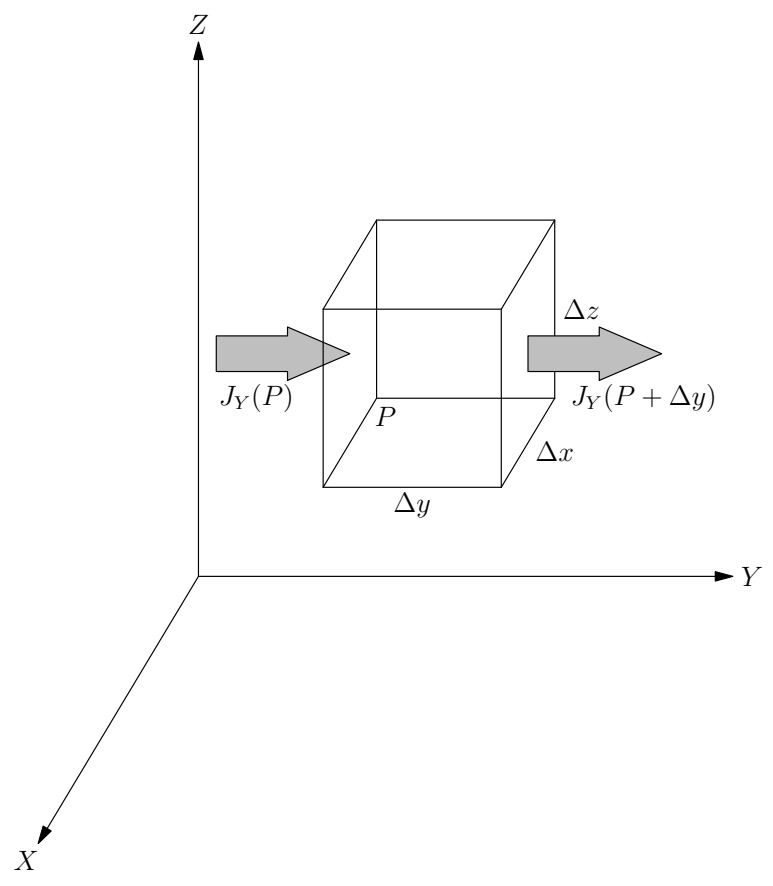

Figure 2.1: Particle flow in Y-direction

The accumulation or loss at the point will be:

$$
\begin{aligned}
\text { Accumulation (or loss) }= & \text { Inflow-Outflow } \\
= & {\left[J_{X}(P+\Delta x)-J_{X}(P)\right] \Delta y \Delta z } \\
& +\left[J_{Y}(P+\Delta y)-J_{Y}(P)\right] \Delta x \Delta z \\
& +\left[J_{Z}(P+\Delta z)-J_{Z}(P)\right] \Delta x \Delta y
\end{aligned} .
$$

The accumulation or loss will cause a change in the concentration at point $P$. If the Taylor expansion for the diffusion flux is considered and higher order terms are neglected eqn. (2.6) can be modified to:

$$
\frac{\partial C}{\partial t} \Delta x \Delta y \Delta z=-\left(\frac{\partial J_{X}}{\partial x}+\frac{\partial J_{Y}}{\partial y}+\frac{\partial J_{Z}}{\partial z}\right) \Delta x \Delta y \Delta z
$$

This can be rewritten in as,

$$
\frac{\partial C}{\partial t}=-\nabla \cdot \mathbf{J}
$$

This equation is called the continuity equation. Substituting the relationship of Fick's 
first law (eqn. (2.2)) in the continuity equation will gives us Fick's second law:

$$
\frac{\partial C}{\partial t}=\nabla \cdot(D \nabla C)
$$

which is also called the diffusion equation. For systems with diffusivities independent of the concentration, this will become:

$$
\frac{\partial C}{\partial t}=D \Delta C=D\left(\frac{\partial^{2} C}{\partial x^{2}}+\frac{\partial^{2} C}{\partial y^{2}}+\frac{\partial^{2} C}{\partial z^{2}}\right)
$$

\subsubsection{Random Walk Theory}

This section will look at a simplified model of random walk consisting of an individual jump process and the Einstein-Smoluchowski Relation. Einstein $[2,3]$ and the simplified relationship provided by Mehrer [1] were reviewed for this section.

Consider jumps between planes 1 and 2 as shown in Fig. 2.2, for interstitial diffusion in a cubic lattice in the $X$-direction, which has a concentration gradient $\partial C / \partial x$. The diffusion flux between these two planes can be written as:

$$
J=\Gamma n_{1}-\Gamma n_{2}
$$

where $n_{1}$ and $n_{2}$ are the number of interstitial atoms in a unit area of each plane and $\Gamma$ is the jump rate of atoms leaving a plane.

Concentrations of the interstitial atom in the planes can be written as,

$$
C_{1}=\frac{n_{1}}{\lambda} \quad C_{2}=\frac{n_{2}}{\lambda}
$$

Concentration gradient can be obtained from the relationship,

$$
C_{1}-C_{2}=-\lambda \frac{\partial C}{\partial x}
$$

Combining eqn. (2.11) and eqn. (2.13), we get,

$$
J=-\lambda^{2} \Gamma \frac{\partial C}{\partial x}
$$

Comparing eqn. (2.1) and eqn. (2.14), we get a relationship for the diffusivity of an individual jump which can be derived as the jump frequency multiplied by the square 


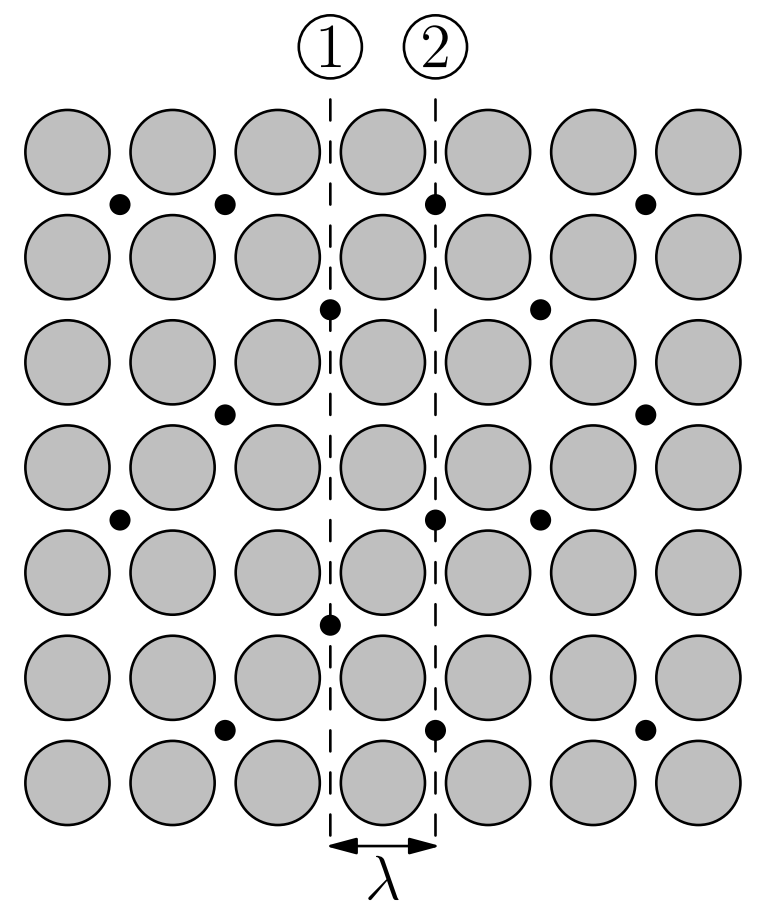

Figure 2.2: Random walk of interstitial solutes

of jump length,

$$
D=\lambda^{2} \Gamma
$$

For a simple cubic lattice this jump can happen in six directions, which would mean that to get the jump rate for a specific direction the total jump rate needs to be divided by 6 .

$$
\Gamma=\frac{1}{6} \Gamma_{\text {total }}
$$

Therefore,

$$
D=\frac{1}{6} \lambda^{2} \Gamma_{\text {total }}
$$

Einstein-Smoluchowski relation considers a collection of such jumps to come up with a more rigorous approach in determining the diffusion coefficients in bulk material. This approach considers a time evolution of a system with independent particle motions done in a large enough time interval so that the motions of the particle is mutually independent (not large enough to observe the motion). Development of the relationship is carried out for the $X$-direction, which is analogous for $Y$ and $Z$ directions.

Take $n$ number of particles in a solution. In a $\tau$ time, each individual particle will 
have a displacement of $x$ in the $X$-direction. This $x$ could be any positive or negative value. If $d n$ number of particles have a displacement of $x+d x$ after the time interval $\tau$,

$$
d n=n \varphi(x) d x
$$

$\varphi(x)$ is a probability density function for the displacement of the particles which is symmetric about $x=0$. Hence,

$$
\varphi(x)=\varphi(-x)
$$

For any probability,

$$
\int_{-\infty}^{+\infty} \varphi(x) d x=1
$$

For small time intervals, this function is localized at small $x$ values $(x \rightarrow 0)$. If the concentration at $\bar{x}$ in time $t$ is $C(\bar{x}, t)$, we can find the total number of particles in a $d \bar{x}$ length (for a unit area normal to the $X$-direction)

$$
C(\bar{x}, t+\tau) d \bar{x}=d \bar{x} \int_{-\infty}^{+\infty} C(\bar{x}+x, t) \varphi(x) d x
$$

From Taylor expansion for small $x$ and $\tau$ and ignoring the relevant higher order terms we get:

$$
\frac{\partial C}{\partial t}=\frac{1}{\tau}\left(\frac{\partial C}{\partial \bar{x}} \int_{-\infty}^{+\infty} x \varphi(x) d x+\frac{\partial^{2} C}{\partial \bar{x}^{2}} \int_{-\infty}^{+\infty} \frac{x^{2}}{2} \varphi(x) d x\right)
$$

Comparing this with the Fick's second law (eqn. (2.9)) in the $X$-direction we can identify that the diffusion coefficient can be expressed as:

$$
D_{X}=\frac{1}{2 \tau} \int_{-\infty}^{+\infty} x^{2} \varphi(x) d x
$$

The first order term is called the drift term which becomes naught in the absence of a driving force such as a stress gradient or a temperature gradient. Generally, the $k^{\text {th }}$ moment of $x$ is denoted by $\left\langle x^{k}\right\rangle$.

$$
\int_{-\infty}^{+\infty} x^{k} \varphi(x) d x=\left\langle x^{k}\right\rangle
$$


Hence,

$$
D_{X}=\frac{\left\langle x^{2}\right\rangle}{2 \tau}
$$

Similarly, for $Y$ and $Z$ directions:

$$
D_{Y}=\frac{\left\langle y^{2}\right\rangle}{2 \tau}, \quad D_{Z}=\frac{\left\langle z^{2}\right\rangle}{2 \tau}
$$

$\left\langle x^{k}\right\rangle$ can be obtained by taking the average of the $x^{k}$ values for many particles,

$$
\left\langle x^{k}\right\rangle=\frac{\sum_{p=1}^{n} x_{p}^{k}}{n}
$$

If the random walk of a particle in a lattice is considered, diffusion will consist of many individual jumps. Then the total displacement in the $X$-direction $x$ consisting of $N$ jumps can be written as:

$$
x=\sum_{i=1}^{N} x_{i}
$$

The square of the displacement will be:

$$
x^{2}=\sum_{i=1}^{N} x_{i}^{2}+2 \sum_{i=1}^{N-1} \sum_{j=i+1}^{N} x_{i} x_{j} \quad(\text { for } \neq j)
$$

If the average of a large number of atoms is taken:

$$
\left\langle x^{2}\right\rangle=\left\langle\sum_{i=1}^{N} x_{i}^{2}\right\rangle+2\left\langle\sum_{i=1}^{N-1} \sum_{j=i+1}^{N} x_{i} x_{j}\right\rangle \quad(\text { for } \neq j)
$$

The $x_{i} x_{j}$ term is relevant for jumps which has a dependency on the previous jumps. For diffusion with no memory effect such as interstitial diffusion in dilute solutions, the average values of $\left\langle x_{i} x_{j}\right\rangle \rightarrow 0$. Hence:

$$
\left\langle x^{2}\right\rangle=\left\langle\sum_{i=1}^{N} x_{i}^{2}\right\rangle
$$

These type of diffusions are called Markov sequences/chains or uncorrelated random jumps. Hence, the diffusion coefficient of uncorrelated random walks can be 


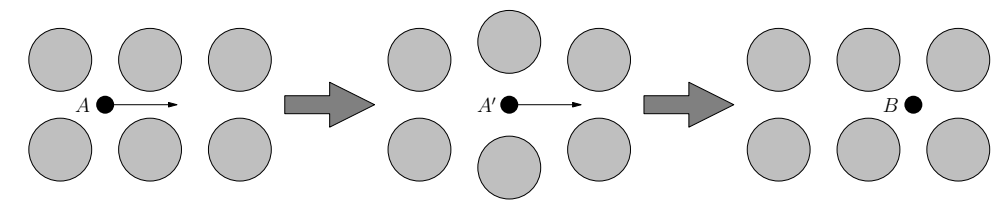

Figure 2.3: Diffusion of an interstitial atom

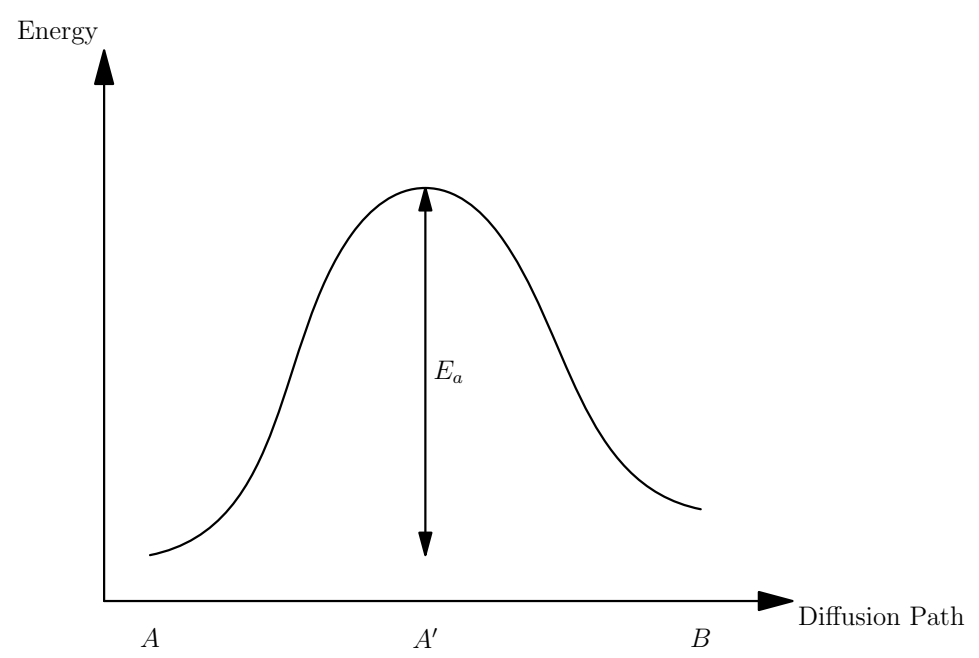

Figure 2.4: Potential energy landscape for the diffusion in Fig. 2.3

written as:

$$
D_{X}=\frac{\left\langle\sum_{i=1}^{N} x_{i}^{2}\right\rangle}{2 \tau}
$$

\subsubsection{Transition States}

Transition state theory as explained by Eyring [4], states that that there exists a state (transition state) where reactants combine to form activated complexes at a higher energy before forming the final products. If we look at the diffusion of an interstitial atom, for the transition from stable $A$ point to stable $B$ (see Fig. 2.3), there is a transition stage $A^{\prime}$ which has a higher energy than both $A$ and $B$ which needs to be passed. The potential energy variation for this transition is shown in Fig. 2.4, which is referred to as the potential energy landscape. The minimum energy that needed to be exceeded to reach the transition state $\left(A^{\prime}\right)$ is called the activation energy $\left(E_{a}\right)$. 


\subsubsection{Arrhenius Relationship}

Arrhenius equation [5] considers the temperature dependence of the reaction rate. This relationship is followed in most cases, but not always [1].

$$
k(T)=k_{0} \exp \left(\frac{-E}{R T}\right)
$$

where $k_{0}$ is a constant, temperature-independent part of the rate, while the temperature-dependent exponential part consists of $E$ which is the activation barrier and $R$ is the gas constant which is replaced by the Boltzmann constant $k_{B}$ when dealing with atomic units $(e V)$. For the sake of clarity and consistency with previous sections, reaction rate $k(T)$, constant $k_{0}$, and activation energy $E$ is replaced by $\Gamma$, $E_{a}$, and $\Gamma_{0}$.

$$
\Gamma=\Gamma_{0} \exp \left(\frac{-E_{a}}{k_{B} T}\right)
$$

Combining eqn. (2.17) and eqn. (2.34) we get:

$$
D=\frac{1}{6} \lambda^{2} \Gamma_{0} \exp \left(\frac{-E_{a}}{k_{B} T}\right)
$$

Here, we can see that the diffusivity comes with a temperature independent component, let's call it $D_{0}$, and a temperature dependent exponential part.

$$
D_{0}=\frac{1}{6} \lambda^{2} \Gamma_{0}
$$

Muta et al. [6] have carried out an ab-initio calculation for the diffusion of $\mathrm{H}$ in zirconium oxide and has equated the attempt frequency to the oscillation frequency of the $\mathrm{H}$ atom $v_{0}$ in the diffusion direction.

$$
\Gamma_{0}=v_{0}=\frac{1}{2 \pi} \sqrt{\frac{2 k}{m}}
$$

$m$ is the mass of a $\mathrm{H}$ atom and $k$ is obtained as the curvature of the energy landscape as shown in Fig. 2.5.

Hence, diffusivity can ultimately be derived using these relationships, using curvature $k$ and the activation energy $E_{a}$ from the energy landscapes (see Fig. 2.5). 


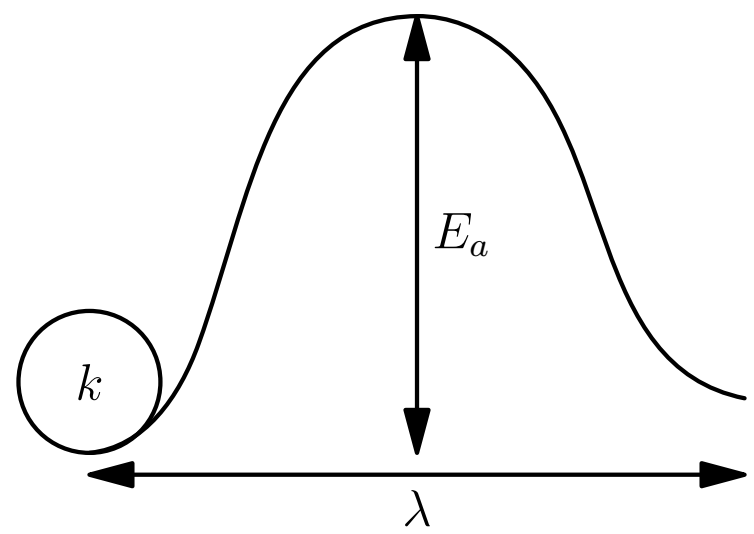

Figure 2.5: Energy landscape of a diffusion step

$$
D=\frac{1}{12 \pi} \lambda^{2} \sqrt{\frac{2 k}{m}} \exp \left(\frac{-E_{a}}{k_{B} T}\right)
$$

While this approach gives a pre-exponent factor for the rate constant, it is assumed that oscillations $\mathrm{H}$ atoms only happens in the direction of the transition. This approach is also computationally expensive and ignores the effect on rate constants from zero-point energy and quantum tunnelling. Fermann and Auerbach [7] has presented the semiclassically corrected harmonic transition state theory which addresses all these issues. It only required the activation energy and the vibration modes at the initial position and saddle point to provide a more accurate estimate for the hopping rates. We have shown the approach for determining the hopping rates using the semi-classically corrected harmonic transition state theory in Appendix A.

\subsection{Computational Methods}

\subsubsection{Density Functional Theory}

Density Functional Theory (DFT) derives the properties of ion-electron systems from their electron density. Compared to wave function approaches, this has the advantages of involving a physical property and being independent of the number of electrons. Wave functions and electron density is used similar to the shape functions in finite element methods. DFT was introduced in Kohn \& Sham [8] and Hohenberg \& Kohn [9] with the use of Slater determinant [10], for which Walter Kohn obtained the Nobel prize in 1998. DFT is considered to have a good balance between the accuracy and 
computational power required. Østli [11], Turgut [12], Tadmor \& Miller [13], and Cuevas [14] were helpful in understanding the related theories.

For an ion-electron system, the physical state of the system can be explained using the Schrödinger's equation

$$
\hat{H} \Psi\left(\mathbf{R}_{\mathbf{I}}, \mathbf{r}_{\mathbf{i}}\right)=E \Psi\left(\mathbf{R}_{\mathbf{I}}, \mathbf{r}_{\mathbf{i}}\right)
$$

$\Psi$ is the quantum mechanical wave function explained with respect to the positions of nuclei $\left(\mathbf{R}_{\mathbf{I}}\right)$ and electrons $\left(\mathbf{r}_{\mathbf{i}}\right) . E$ is the energy of the system and $\hat{H}$ is the Hamiltonian operator defined as,

$$
\begin{aligned}
\hat{H}= & -\sum_{i} \frac{1}{2} \nabla^{2} \mathbf{r}_{\mathbf{i}}-\sum_{I} \frac{1}{2 M_{Z}} \nabla^{2} \mathbf{R}_{\mathbf{I}}-\sum_{i} \sum_{I} \frac{Z_{I}}{\left|\mathbf{R}_{\mathbf{I}}-\mathbf{r}_{\mathbf{i}}\right|} \\
& +\sum_{i} \sum_{j>i} \frac{1}{\left|\mathbf{r}_{\mathbf{i}}-\mathbf{r}_{\mathbf{j}}\right|}+\sum_{I} \sum_{J>I} \frac{Z_{I} Z_{J}}{\left|\mathbf{R}_{\mathbf{I}}-\mathbf{R}_{\mathbf{J}}\right|}
\end{aligned}
$$

Here the Hartree atomic units are used. The first two terms of the Hamiltonian describe the kinetic energy of electrons and the nuclei respectively. The next term show the attraction between electrons and the nuclei and the final two terms shows the energy from repulsion of electrons and nuclei respectively.

Born \& Oppenheimer [15], Hartree [16], and Fock [17] provided with approximations to the Schrödinger's equation in developing simplified solution methods using wave functions. Thomas [18] and Fermi [19] made initial attempts in using electron density from two independent studies. These ignores the effect of exchange and correlation effects as stated by the Pauli Exclusion Principle. Kohn \& Sham [8] and Hohenberg \& Kohn [9] finally came up with the density functional theory we are using today, with good approximation for the Hamiltonian using electron densities.

\section{K-point sampling}

For many calculations, values of functional of electron density needs to be integrated over Brillouin-zones (reciprocal space), which in a computer is generally done discretely. This is generally carried out by evaluating the functions at specific points of Brillouin-zones called K-points, and combining them to get the values for the total space similar to that of integration points in finite element methods. For hexagonal lattices, a Gamma centered grid of K-points is generally recommended as It gives a 
faster convergence than a standard Monkhorst-Pack grid, which considers K-points with equal spacing [20].

\subsubsection{Nudged Elastic Band Method}

Nudged Elastic Band (NEB) method is an efficient way of identifying the minimum energy path for the rearrangement of a system of atoms, given that the initial and the final states are known. The minimum energy path is essentially important in determining the reaction coordinates (energy landscapes) for chemical reactions, changes in conformation of molecules and diffusion processes in solids. The reaction coordinates are required to determine the activation barriers of different processes. NEB can be used in conjunction with both DFT methods and empirical potentials.

NEB method was developed in Mills \& Jónsson , [21], Mills et al. [22], and Jónsson et al. [23], then improved in Henkelman \& Jónsson [24] to get rid of the kinks observed in the original formulation. NEB a set of images between the starting and the ending states (called an elastic band), and "nudges" the images in the directions of the force on each image until the force is minimized. A spring interaction is acting between the images to ensure continuity.

\section{Climbing Image Nudged Elastic Band Method}

The NEB provides the images with a uniform distribution of images. Hence if the resolution for the minimum energy path is kept at a smaller value the true saddle point can be lost which could lead to an underestimate of the activation energy. Henkelman et al. [25] proposes that modifying the force on the image with the highest energy (identified after a few iterations), such that it pulls at the saddles point, while the forces on other images continues to retain the shape of the minimum energy path, will make the image with the highest energy converge on the saddle point.

\subsubsection{Vienna Ab-initio Simulation Package}

Vienna Ab-initio Simulation Package (VASP) [26-28] is a complex package, carrying out molecular dynamic simulations, which provides solutions to the Kohn-Sham functional using pseudopotentials or projector-augmented wave (PAW) method. For NEB calculations, the customization by Henkelman Group [29], is used for the VASP code in order to activate the climbing image NEB method. 
VASP mainly utilizes 4 input files POSCAR, INCAR, KPOINTS and POTCAR [20]. POSCAR file provides the initial locations of the atoms in the system and the arrangement of the supercell. The INCAR file contains the instructions on what to do and how to do it. VASP contains many parameters which can be changed, but even if they are not specified, the default values will be assigned. KPOINTS file contains the k-point coordinates and weights which can be explicitly stated, or generated automatically. POTCAR has pseudopotentials for all the species of atoms containing in the system.

\subsubsection{Kinetic Monte Carlo Simulation}

This section contains a brief description of the history and procedure of the Kinetic Monte Carlo (KMC) simulation method. Voter [30] was used in understanding the history and the procedure of the method.

$\mathrm{KMC}$ is a member of the family of Monte Carlo simulations, which solves problems with the use of random numbers as the name comes from Monte Carlo, Monaco which is famous for gambling. These methods have emerged with the advancement of computers in 1940s and 1950s. The Metropolis algorithm is the most famous of the Monte Carlo algorithms.

In 1960s a Monte Carlo method was introduced which looks at the evolution of a system from a state to state. The name Kinetic Monte Carlo Simulation came to being only in the 1990s. KMC is considered to resolve the time scale problem of molecular dynamic simulations. Molecular dynamic simulations advance the system with time using equations of motion. This requires the time integration to be done in

very small steps $\left(10^{-15} s\right)$, making the total achievable time evolution of the system to be less than 1 microsecond. In case of studies such as diffusion. KMC provides a mean of overcoming this problem by mapping the system as a series of state to state transitions without considering the motions in between them. This allows the study of longer time periods.

\section{KMC procedure}

Since the escape from a system is not dependent on the motions it carried out inside a state, the probability that the system has not yet escaped the state will have an exponentially decaying relationship with time. The probability that the system has 
survived this state at a time $t$ can be given by:

$$
p_{\text {survive }}(t)=\exp (-k t)
$$

where, $k$ is the rate constant for the reaction. For the case of diffusion, this can be estimated with the use of Arrhenius relationship as mentioned in eqn. (2.34). Hence, the probability that a system has escaped by a given time $t$ can be obtained as:

$$
p_{\text {escape }}(t)=1-\exp (-k t)
$$

Probability distribution for the time for escape $p(t)$ can be obtained by the time derivative of eqn. (2.42).

$$
p(t)=k \exp (-k t)
$$

The average time $\tau$ taken for a system to escape from a state, can be found as:

$$
\tau=\int_{0}^{\infty} t p(t) d t=\frac{1}{k}
$$

According to this relationship it can be seen that systems with high rate constants will occupy the respective state for a shorter time. Hence, they will have a high probability of escaping the state.

Take that the system is in a state $i$ which has $N$ possible states to transfer to. Define the rate of transition from $i$ to the $j$ state as $k_{i, j}$. Then the sum of total rate constants will be:

$$
k_{i, t o t}=\sum_{j}^{N} k_{i, j}
$$

Next means to map the random numbers generated to select the escape route taken needs to be developed. This is generally carried out as illustrated in Fig. 2.6. The probability of escaping to state $j$ is proportional to $k_{i, j}$. The rates are arranged (in no particular order) with each path having a range equal to its rate constant $k_{i, j}$. A random number $r$ is selected from $(0,1]$ and we check the escape route which will contain the value $r$ in $k_{\text {tot }}$. This also can be carried out using an array $s_{i, j}$, where:

$$
s_{i, j}=\sum_{q}^{j} k_{i, q}
$$




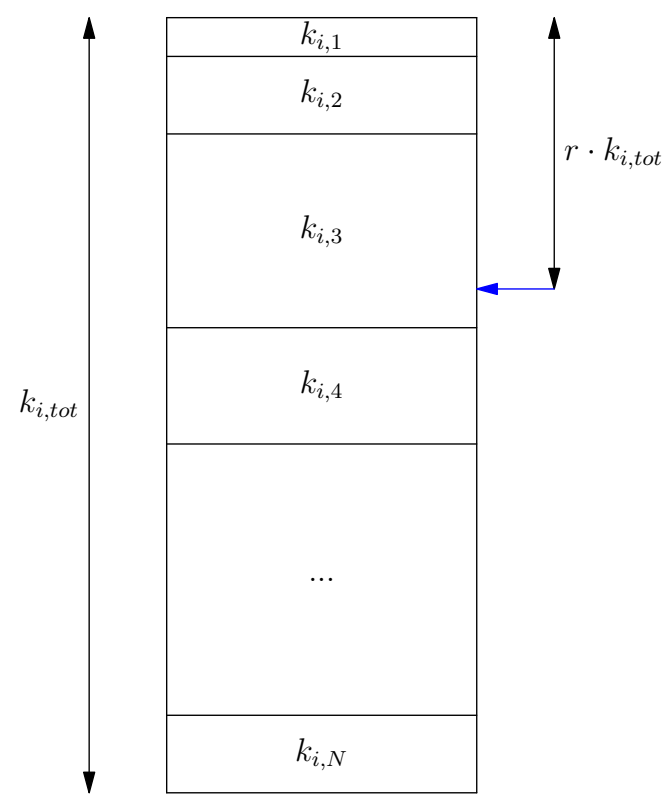

Figure 2.6: Illustration of picking the escape path

The first $j$ for which the value $s_{i, j}$ is exceeded will be for the escape path selected. Once the path is identified, the time taken for the transition is estimated with another random variable $\bar{r}$ selected from $(0,1]$, as taken as shown in eqn. (2.47). Once the escape path and the time taken for the escape is calculated, the KMC step is completed. Then the escape from state $j$ needs to be considered. Once the required number of steps, or time has elapsed, the KMC procedure will be over.

$$
\Delta t=\frac{-\ln (\bar{r})}{k_{i, t o t}} .
$$

\section{Bibliography}

[1] H. Mehrer, Diffusion in Solids: Fundamentals, Methods, Materials, DiffusionControlled Processes. Vienna, Austria: Springer-Verlag Berlin Heidelberg, 1 ed., 2007.

[2] A. Einstein, "Über die von der molekularkinetischen theorie der wärme geforderte bewegung von in ruhenden flüssigkeiten suspendierten teilchen," Annalen der Physik, vol. 322, no. 8, pp. 549-560, 1905.

[3] A. Einstein, "On the movement of small particles suspended in stationary liquids required by the molecular-kinetic theory of heat - on the random motion of particles suspended in a liquid and their relationship to diffusion," in The Swiss Years: Writings, 1900-1909 (English translation supplement) (J. Stachel, D. C. Cassidy, J. Renn, and R. Schulmann, eds.), vol. 2 of The Collected Papers of 
Albert Einstein, ch. 16, pp. 130-134, Princeton, New Jersey: Princeton University Press, 1990.

[4] H. Eyring, "The activated complex in chemical reactions," The Journal of Chemical Physics, vol. 3, pp. 107-115, 1935.

[5] S. Arrhenius, "On the reaction velocity of the inversion of cane sugar by acids," Zeitschrift für Physikalische Chemie, vol. 4, p. 230-234, 1889.

[6] H. Muta, Y. Etoh, Y. Ohishi, K. Kurosaki, and S. Yamanaka, "Ab initio study of hydrogen diffusion in zirconium oxide," Journal of Nuclear Science and Technology, vol. 49, no. 5, pp. 544-550, 2012.

[7] J. T. Fermann and S. Auerbach, "Modeling proton mobility in acidic zeolite clusters: II. room temperature tunneling effects from semiclassical rate theory," J. Chem. Phys., vol. 112, pp. 6787-6794, 2000.

[8] W. Kohn and L. J. Sham, "Self-consisten equations includingexchange and correlation effects," Physical Review, vol. 140, p. A1133-A1138, 1965.

[9] P. Hohenberg and W. Kohn, "Inhomogeneous electron gas," Physical Review B, vol. 7, p. 1912-1919, 1973.

[10] J. C. Slater, "A simplification of the hartree-fock method," Physical Review, vol. 81, p. 385-390, 1951.

[11] P. Østli, Density Functional Theory Studies of Precipitate Interfaces in Aluminium Alloys, with Focus on $\theta^{\prime}-\mathrm{Al}_{2} \mathrm{Cu}$. PhD thesis, Norwegian University of Science and Technology, 2016.

[12] S. Turgut, "Hartree-fock approximation." Lecture Notes, 2005.

[13] E. B. Tadmor and R. E. Miller, "Quantum mechanics of materials," in Modelling Materials - Continuum, Atomistic and Mutiscale Techniques, ch. 4, p. 153-236, Cambridge University Press, 2011.

[14] J. C. Cuevas, "Introduction to density functional theory." Lecture Notes, 2011.

[15] M. Born and R. Oppenheimer, "Zur quantentheorie de molekeln," Annalen der Physik, vol. 389, p. 457-484, 1927.

[16] D. R. Hartree, "The wave mechanics of an atom with a non-coulomb central field. part i. theory and methods," Mathematical Proceedings of the Cambridge Philosophical Society, vol. 24, pp. 89-110, 1928.

[17] V. Fock, "Näherungsmethode zur lösung des quantenmechanischen mehrkörperproblems," Zeitschrift für Physik, vol. 61, p. 126-148, 1930.

[18] L. H. Thomas, "The calculation of atomic fields," Mathematical Proceedings of the Cambridge Philosophical Society, vol. 23, p. 542-548, 1927. 
[19] E. Fermi, "Un metodo statistico per la determinazione di alcune priorieta dell'atome," Rendicondi Accademia Nazionale de Lincei, vol. 32, p. 602-607, 1927.

[20] G. Kresse, M. Marsman, , and J. Furthmüller, Vienna Ab-initio Simulation Package VASP the GUIDE. 2014.

[21] G. Mills and H. Jónsson, "Quantum and thermal effects in $\mathrm{H}_{2}$ dissociative adsorption: Evaluation of free energy barriers in multidimensional quantum systems," Physical Review Letters, vol. 72, no. 7, pp. 1124-1127, 1994.

[22] G. Mills, H. Jónsson, and G. K. Schenter, "Reversible Work Transiton State Theory: Application to Dissociative Adsorption of Hydrogen," Surface Science, vol. 324, no. 2-3, pp. 305-337, 1995.

[23] H. Jónsson, G. Mills, and K. W. Jacobsen, "Nudged elastic band method for finding minimum energy paths of transitions," in Classical and Quantum Dynamics in Condensed Phase Simulations, ch. 16, pp. 385-404, World Scienific, 1998.

[24] G. Henkelman and H. Jónsson, "Improved tangent estimate in the nudged elastic band method for finding minimum energy paths and saddle points Improved tangent estimate in the nudged elastic band method for finding minimum energy paths and saddle points," Journal of Chemical Physics, vol. 113, pp. 9978-9985, 2000 .

[25] G. Henkelman, B. P. Uberuaga, and H. Jónsson, "Climbing image nudged elastic band method for finding saddle points and minimum energy paths," Journal of Chemical Physics, vol. 113, no. 22, pp. 9901-9904, 2000.

[26] G. Kresse and J. Hafner, "Ab initio molecular dynamics for liquid metals," Physical Review B, vol. 47, no. 1, pp. 558-561, 1993.

[27] G. Kresse and J. Furthmüller, "Efficiency of ab-initio total energy calculations for metals and semiconductors using a plane-wave basis set," Computational Materials Science, vol. 6, no. 1, pp. 15-50, 1996.

[28] G. Kresse and J. Furthmüller, "Efficient iterative schemes for $<\mathrm{i}>$ ab initio $</ \mathrm{i}>$ total-energy calculations using a plane-wave basis set," Physical Review B, vol. 54, no. 16, pp. 11169-11186, 1996.

[29] "Nudged Elastic Band - Transition State Tools for VASP." http://theory. cm.utexas.edu/vtsttools/neb.html. Accessed: 2017 Jul. 08.

[30] A. F. Voter, "Introduction To the Kinetic Monte Carlo Method," in Radiation Effects in Solids (K. E. Sickafus, E. A. Kotomin, and B. P. Uberuaga, eds.), ch. 1, pp. 1-23, Dordrecht: Springer Netherlands, 2007. 


\section{Chapter 3}

\section{Multi-scale approach for determining hydrogen diffusivity in zirconium ${ }^{1}$}

Using the theories and computational methods presented in Chapter 2 we developed a multi-scale model, utilising first-principles calculations and KMC simulations, to model $\mathrm{H}$ diffusion in lattice structures. This chapter includes the paper which we produced to validate the model using the results available in the literature for $\mathrm{H}$ diffusion in Zr. We successfully validated the model, which allowed us to extend it to other studies. We also investigated the diffusion process through probability of each jump to occur, and the mean square displacement of the jumps, to explain that $\mathrm{H}$ displays a closely isotropic diffusion in the Zr lattice.

\subsection{Introduction}

Hydrogen induced degradation of material such as metals and piezoelectric material is an area which has attracted considerable research activity [1-6]. Zirconium, whose alloys are used as fuel cladding, pressure tubes, fuel channels, and fuel spacer grid material in nuclear reactors, has been a main candidate for these studies, as any failure of Zr elements would be catastrophic [7-9]. The harsh environmental conditions of nuclear reactors are very favorable for creation of atomic hydrogen, which absorbs into metals more easily than hydrogen in the molecular state. Low hydrogen solubility in zirconium causes most of the absorbed hydrogen to be precipitated as brittle zirconium hydrides, which favor formation at grain boundaries, crack tips, and

\footnotetext{
${ }^{1}$ Contains the work in Manura Liyanage, Ronald Miller, and R. K. N. D. Rajapakse, "Multiscale approach for determining hydrogen diffusivity in zirconium", Modelling and Simulation in Materials Science and Engineering, vol. 26, no. 8, pp. 085002 1-20, 2018.
} 
places under tensile stress [10]. If enough hydrides accumulate in a crack tip which is under a tensile stress, this could lead to a premature failure called Delayed Hydride Cracking (DHC) [11-14], which is a main mechanism of failure in zirconium pressure tubes.

Diffusion of hydrogen in zirconium has been studied with experimental and theoretical approaches, producing an array of somewhat variable results. This paper gives a comprehensive description of a multiscale approach to the problem. Combining first principles studies and Kinetic Monte Carlo simulations (KMC), we compute the diffusivity of hydrogen in zirconium while also verifying the model to be extended for other diffusion studies. Zhang et al. [15] used a similar approach to determine the hydrogen diffusivity in zirconium. Comparative to that, we give comprehensive detail on determining rate constants for the transition steps for hydrogen in hcp zirconium, using the energy landscape of their motion in the hexagonal close packed (hcp) zirconium lattice calculated from first principles. Also, in the current study these calculations are carried out without any dimensional constraints to simulate a stress free condition. These results are then used as input in KMC simulations utilizing a periodic cell of the crystal, which reduces the effort in KMC calculations, to estimate diffusivity of hydrogen in zirconium. We also look closely at the diffusion steps of atomic hydrogen motion in the zirconium lattice, to identify the reasons for the nearly isotropic behavior of hydrogen diffusivity in the anisotropic hcp zirconium lattice in the current study.

Through this work we propose and validate a modelling approach which can successfully estimate diffusivity of a particle for which we know the clearly defined diffusion steps available to it (In Sec. 3.5.4 and Fig. 3.14, we elaborate on the procedure followed in the current study).

\subsection{Hydrogen in Zirconium}

Hydrogen uptake is kept to a minimum during the manufacturing stages of zirconium pressure vessels due to its detrimental nature. Nonetheless, hydrogen can get absorbed in its atomic form [16] through the oxidation reaction of zirconium with high temperature water. Hydrogen added to collect the oxygen produced during the radiolytic decomposition of $\mathrm{D}_{2} \mathrm{O}$, hydrogen impurities in added nitrogen and carbon dioxide gases between the reactor tubes, and Zirconium hydrides added as moderators 
for the nuclear reactions during operations also supply atmoic hydrogen to zirconium pressute tubes $[16,17]$.

Absorbed hydrogen has been known to cause hydrogen embrittlement in metals through several mechanisms. Formation of brittle hydrides is expected to be the main mechanism responsible for failure in zirconium $[3,4,6]$ leading to loss of toughness and DHC. The level of hydrogen solubility in zirconium has been observed by many researchers with some variability. It has been estimated that the hydrogen solubility in zirconium is in the range $0.27-1.16 \mathrm{~atm} \%$ [18-20] in reactor operation conditions. Blackmur et al. [7] states that the highest hydrogen solubility cannot exceed $3 \%$ atm for these conditions. Christensen et al. [10] observed that the solubility drops to 0.000054 atm $\%$ at $25{ }^{\circ} \mathrm{C}$ and Kumar [16] has found maximum solubility levels of $6 \mathrm{~atm} \%, 0.70 \mathrm{~atm} \%$, and $0.10 \mathrm{~atm} \%$ at $500^{\circ} \mathrm{C}, 300{ }^{\circ} \mathrm{C}$, and room temperature respectively. This shows the high temperature dependence of solubility, which implies that bulk of the hydrogen absorbed in the high temperature operational stages would precipitate as hydrides when it cools down.

Once the solubility of hydrogen is exceeded, hydrides start to form mainly in the grain boundaries as pallets or needle shaped precipitates [7] as seen from the phase diagram in Fig. 3.1. In this figure, the $\alpha$ and $\beta$ states refer to the dissolved hydrogen in hcp and bcc (body centered cubic) zirconium crystals and $\gamma, \delta$ and $\varepsilon$ are the brittle hydride phases.

\subsection{Hydrogen Diffusion in Zirconium}

Hydrogen has very high transportation capabilities in its atomic state due to its small size. It mainly follows an interstitial mechanism during diffusion. Zirconium predominantly has a hexagonal close packed crystal structure (see Fig. 3.2) at temperatures below $862{ }^{\circ} \mathrm{C}$ (which includes reactor operational temperatures and ambient temperature). It has been found that hydrogen occupies tetragonal and octahedral interstitial sites [18] as shown in Fig. 3.3, represented by black and red colored spheres respectively. There are four tetrahedral sites and two octahedral sites per unit cell. Of these two types of occupancy sites, hydrogen energetically prefer tetrahedral sites by a small margin. Comparing the binding energy of these sites, Burr et al. [18] and Domain et al. [22] have found a preference for tetrahedral sites of $5.5 \mathrm{~kJ} \mathrm{~mol}^{-1}(0.057 \mathrm{eV} /$ atom $)$ and $8.3 \mathrm{~kJ} \mathrm{~mol}^{-1}(0.086 \mathrm{eV} /$ atom $)$ respectively. Christensen et al. [10] state that the 


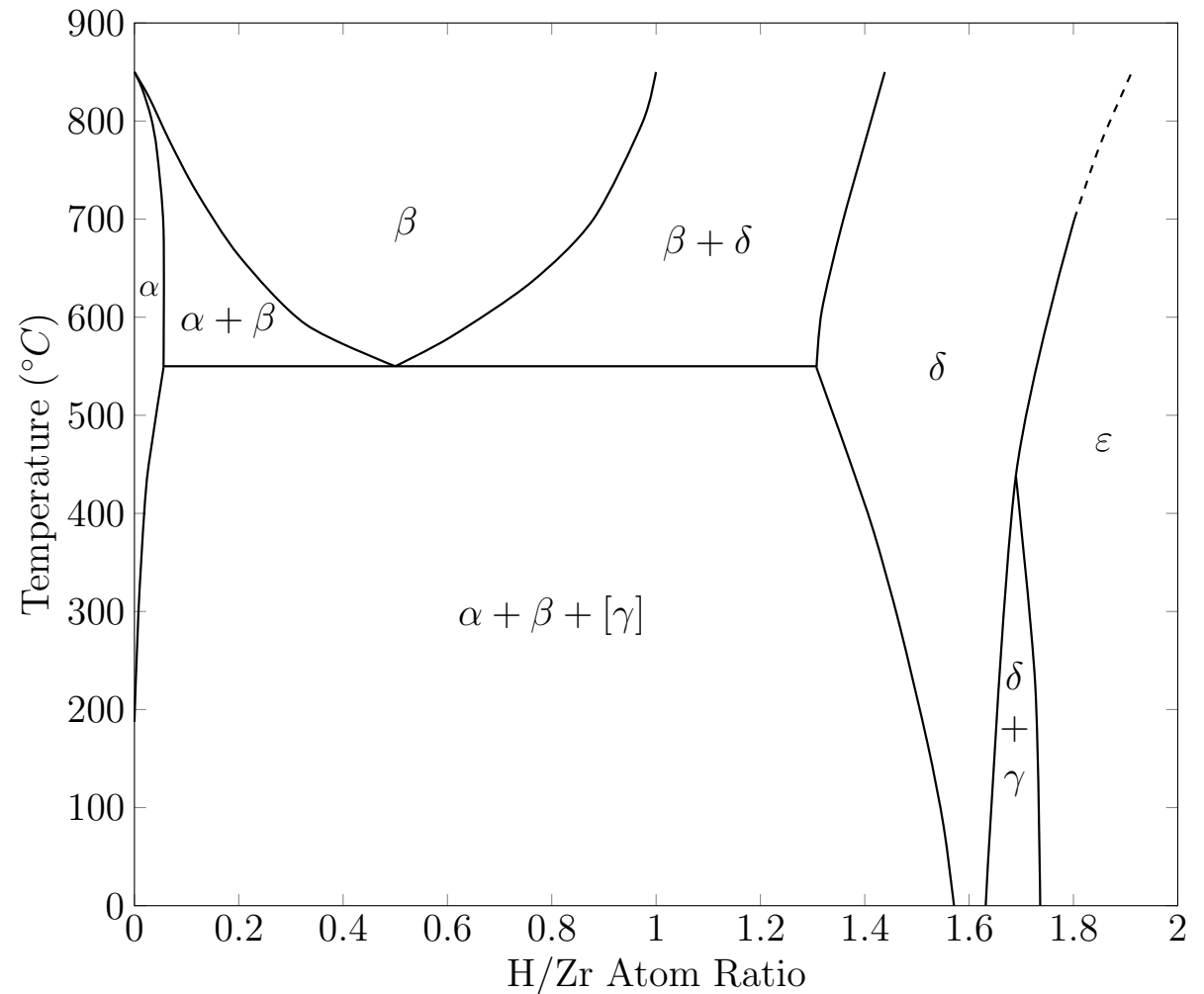

Figure 3.1: Phase diagram for hydrogen in zirconium, where $\alpha$ and $\beta$ are the states with dissolved hydrogen, which mainly occupied interstitial sites, while $\gamma, \delta$ and $\varepsilon$ shows the brittle hydride phases [21] 


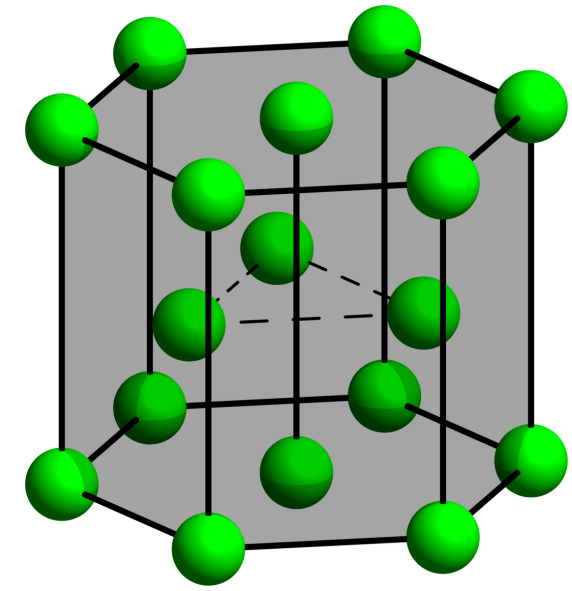

(a) Close packed structure

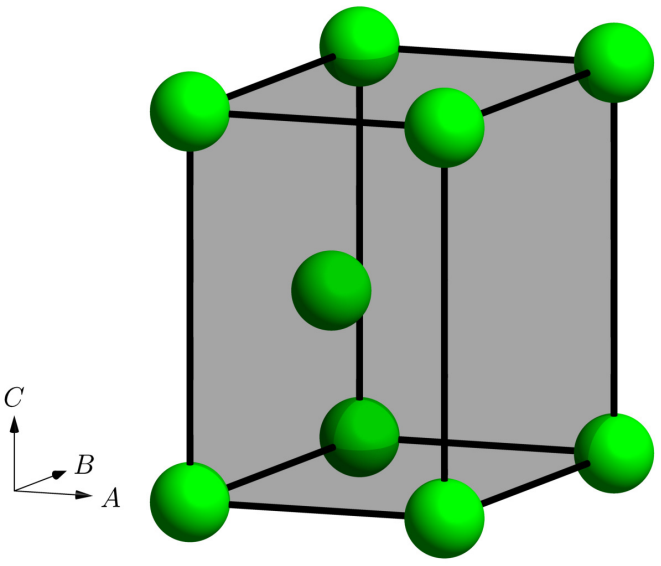

(b) Conventional unit cell

Figure 3.2: Arrangement of atoms in the hexagonal close packed crystal structure

variation of this preference could be due to the temperature where the binding energy of the octahedral site decreases with lowering temperature. Their study shows that at $0 \mathrm{~K}$ the preference for the tetrahedral site is only $0.5 \mathrm{~kJ} \mathrm{~mol}^{-1}(0.0052 \mathrm{eV} /$ atom $)$ which increases to $8.6 \mathrm{~kJ} \mathrm{~mol}^{-1}(0.089 \mathrm{eV} / \mathrm{atom})$ at $600 \mathrm{~K}$. At $600 \mathrm{~K}$ about $6 \%$ of the hydrogen is occupied by octahedral sites, while more hydrogen occupies octahedral sites at lower temperatures.

Table 3.1 shows some of the diffusion coefficients available in literature (the temperature independent component $D_{0}$ and activation barrier $E_{a}$ in the Arrhenius equation, (see eqn. (2.34)). These values shows relatively high variability, with most of the studies being carried out through experimental methods. The activation energy, for the most part, falls between 0.4 and $0.5 \mathrm{eV}$, and the temperature independent diffusion constant $D_{0}$ lies in the range of $1-8 \times 10^{-3} \mathrm{~cm}^{2} / \mathrm{s}$. Though some of the studies were done on alloys of zirconium (zircaloys), Kearns [23] has found that there is no significant effect of the alloying elements on diffusivity. The anisotropy of the diffusion of hydrogen in zirconium is open for debate where some researchers consider the axis direction (C-direction in Fig. 3.2(b)) to have a higher diffusion capacity compared to the basal plane (AB plane in Fig. 3.2(b), which is isotropic) directions. Christensen et al. [10] holds the view that the diffusion of hydrogen in zirconium is isotropic, a view which is also held by McRae et al. [24]. The latter authors also propose that the reason for the variation of diffusivity from previous studies is due 


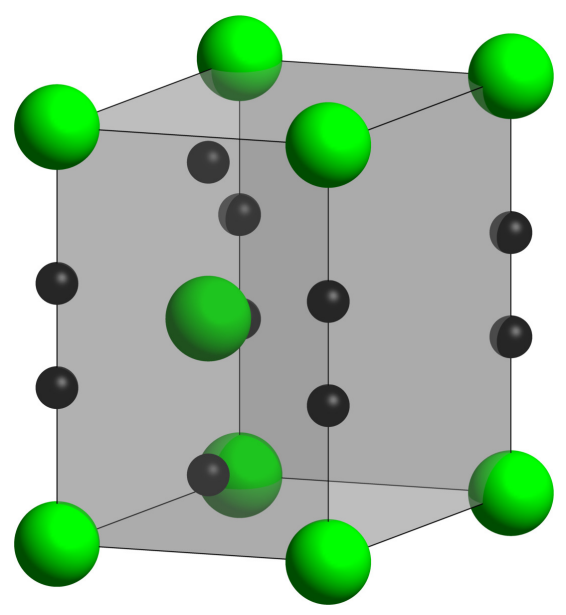

(a) Tetrahedral interstitial sites

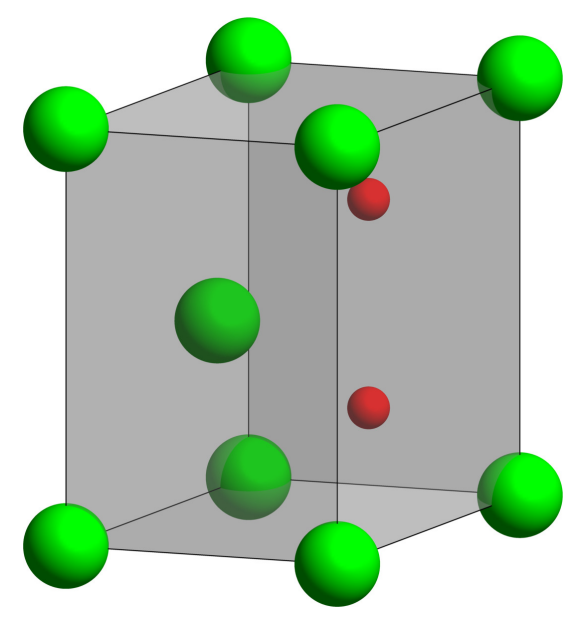

(b) Octahedral interstitial sites

Figure 3.3: Available interstitial site locations for the occupancy of hydrogen in a zirconium unit cell, green color spheres represent the zirconium atoms in the hcp lattice, while black and red color spheres represent tetrahedral and octahedral interstitial sites available for hydrogen occupancy. Some of the tetrahedral sites will be shared between adjacent unit cells

to the driving force by the stress gradients in Onsager's flux theory. It is noticeable that most of the studies which suggest isotropy in hydrogen diffusion in zirconium are based on computational studies while, experimental studies would suggest otherwise.

\subsection{Modelling Approach}

Fick's law is the main law governing diffusion of a particle in a medium, which states that the diffusion flux $(J)$ is proportional to the concentration gradient $(\partial C / \partial x)$ as:

$$
J_{X}=-D \frac{\partial C}{\partial x}
$$

where $D$ the proportional constant called diffusivity, which measures the diffusion capacity of the particle in a certain medium. Diffusivity follows the Arrhenius relationship suggesting a high dependence on the temperature,

$$
D=D_{0} \exp \left(\frac{-E_{a}}{k_{\mathrm{B}} T}\right),
$$


Table 3.1: $D_{0}$ and $E_{a}$ (from eqn. eqn. (2.34)) from previous studies in zirconium obtained through experimental and computational methods

\begin{tabular}{cccc}
\hline References & Details & $D_{0}\left(\mathrm{~cm}^{2} / \mathrm{s}\right)$ & $E_{a}(\mathrm{eV})$ \\
\hline \hline & Longitudinal (experimental) & $7.73 \times 10^{-3}$ & 0.470 \\
Kearns [23] & Transverse (experimental) & $5.84 \times 10^{-3}$ & 0.446 \\
& Radial (experimental) & $7.90 \times 10^{-3}$ & 0.465 \\
Someno [25] & $\alpha$-Zr (experimental 450-700 ${ }^{\circ}$ ) & $4.15 \times 10^{-3}$ & 0.411 \\
& $\beta$-Zr (experimental 870 - 1100 $\left.{ }^{\circ}\right)$ & $7.37 \times 10^{-3}$ & 0.370 \\
Mallett \& Albrecht [26] & Experimental & $7.00 \times 10^{-3}$ & 0.306 \\
Naito [27] & $\alpha$-Zr (experimental) & $6.70 \times 10^{-3}$ & 0.475 \\
Mazzolai \& & Experimental & $5.00 \times 10^{-3}$ & 0.500 \\
Ryll-Nardzewsi [28] & $\alpha$-Zircaloy-2 (experimental) & $2.17 \times 10^{-3}$ & 0.363 \\
Sawatsky [29] & Hydrogen in Zr (experimental) & $1.09 \times 10^{-3}$ & 0.494 \\
Gulbransen \& Andrew [30] & Deuterium in Zr (experimental) & $0.73 \times 10^{-3}$ & 0.494 \\
Siripurapu et al. [31] & Molecular Dynamics (EAM) & $1.69 \times 10^{-2}$ & 0.490 \\
Christensen et al. [10] & Molecular Dynamics (KMC) & $1.13 \times 10^{-3}$ & - \\
Zhang et al. [15] & DFT \& KMC & $5.55 \times 10^{-3}$ & 0.410 \\
\hline
\end{tabular}


where $D_{0}$ is the temperature-independent component of diffusivity, $E_{a}$ is the activation barrier for diffusion, $T$ is the absolute temperature, and $k_{\mathrm{B}}$ is the Boltzmann constant $\left(8.617 \times 10^{-5} \mathrm{eV} / \mathrm{K}\right)$.

The Einstein-Smoluchowski relation $[32,33]$ states that for a large number of particles diffusing in a particular medium with no driving forces such as concentration gradients, its diffusivity can be found by,

$$
D_{X}=\frac{\left\langle x^{2}\right\rangle}{2 t}
$$

where $D_{X}$ is the diffusivity in $X$ direction (which can be extended to $Y$ and $Z$ directions) and $\left\langle x^{2}\right\rangle$ is the mean square displacement in $Z$ direction in $t$ time.

In the case of uncorrelated diffusion, like hydrogen diffusion in zirconium with an interstitial mechanism at low concentrations, the mean square displacement is equal to the sum of the squares of individual displacement steps,

$$
\left\langle x^{2}\right\rangle \approx\left\langle\sum x_{i}^{2}\right\rangle,
$$

which modifies eqn. (3.3) to,

$$
D_{X}=\frac{\left\langle\sum x_{i}^{2}\right\rangle}{2 t} .
$$

Note that while eqn. (3.5) has been found to give faster convergence in KMC simulations, it depends on the validity of the assumption embodied in eqn. (3.4). As we will describe later, this must be used with some caution. In general, diffusivity can always be obtained from eqn. (3.3), but this approach will take a larger number of simulations and might not be practical if many simulations of similar systems are required. In eqn. (3.5) this is modified for uncorrelated diffusions, considering the sum of the squares of displacements for each diffusion step. Here, only the stateto-state transitions are considered, ignoring the motion inside a stable state (energy basin) which will not contribute to diffusion. However, if some transitions have very low barriers compared to others that are available to the diffusion process, they may happen very frequently, hopping back and forth and effectively behaving like motions inside an energy basin. These transitions should be ignored, but eqn. (3.5) will incorrectly include them and give incorrect results. This can be corrected, and the accuracy of eqn. (3.5) restored, using the modified energy basin (pseudo-basin) approach described by Zhang et al. [15]. 


\subsubsection{First Principles Studies}

First principles calculations were done using VASP [34-36] which provides solutions to the Kohn-Sham functional (Density Functional Theory, DFT) using pseudopotentials or the projector-augmented wave (PAW) method. We used the experimental lattice parameters of hcp zirconium $(a=3.23 \AA$ and $c / a=1.593)$ [37] and results from previous DFT studies $[38,39]$ to determine the accuracy of the results of the first principles calculations. We found that generalized gradient approximation using the Perdew, Burke and Ernzerhof (PBE) [40] simplification for estimating exchange correlation energies gives the best agreement to the lattice parameters. A system size of $4 \times 4 \times 3$ unit cells (in a, b, and c directions of Fig. 3.2(b)) with periodic boundary conditions, a K-point sampling mesh density of $5 \times 5 \times 5$, and a $250 \mathrm{eV}$ cut-off energy for plane wave basis set was used in the calculation which gives a convergence of less than $1 \mathrm{meV}$.

Total energies of the occupancy locations of tetrahedral and octahedral sites were first determined as means of model validation. From the results, we determined that hydrogen has an energy preference for the tetrahedral site (energy of a hydrogen atom in an octahedral site - energy of a hydrogen atom in a tetrahedral site) of $0.028 \mathrm{eV}$. This value is relatively low ompared to the values from previous studies (0.089 eV [13], $0.063 \mathrm{eV}$ [15], $0.086 \mathrm{eV}$ [17], and $0.057 \mathrm{eV}$ [18]). Differences could be due to the temperature effects as suggested by Christensen et al. [10] and the fact that the current study allow the system to relax without any dimensional constraints (i.e. simulations are at zero stress instead of constant volume). This takes the suggestions by McRae et al. [24] to allow for stress-free diffusion.

We carried out Nudged Elastic Band (NEB) [41-44] calculations in VASP, to determine the energy landscape of hydrogen diffusion paths. Fig. 3.4 shows the generic energy variation along a diffusion path. The rate constant $(\Gamma)$ for each diffusion step follows an Arrhenius form,

$$
\Gamma=\Gamma_{0} \exp \left(\frac{-E_{a}}{k_{\mathrm{B}} T}\right),
$$

with a temperature independent component $\left(\Gamma_{0}\right)$ and an activation barrier $\left(E_{a}\right)$ similar to eqn. (3.2). The activation energy is determined as the additional energy needed to overcome the highest energy point of the path from the initial state $\left(E_{a}\right.$ shown in Fig. 3.4). Muta et al. [45] has estimated $\Gamma_{0}$ from the oscillation frequency of hydrogen 


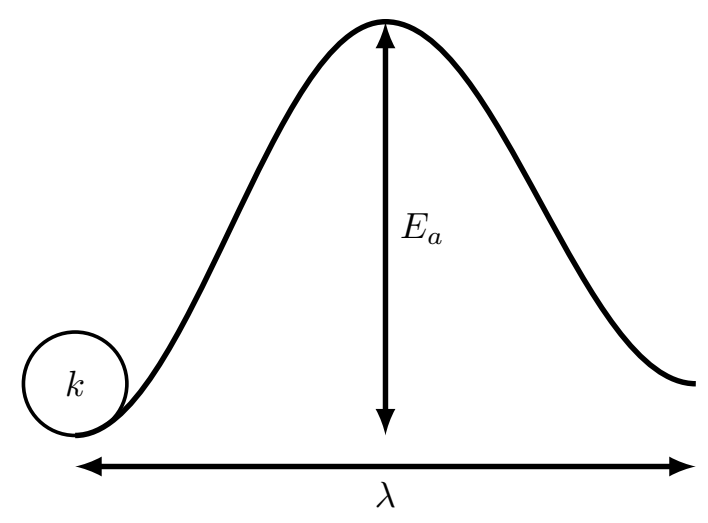

Figure 3.4: Typical energy variation along the diffusion path

$(\nu)$ in the energy basin in the respective direction as,

$$
\Gamma_{0}=\nu_{0}=\frac{1}{2 \pi} \sqrt{\frac{2 k}{m}},
$$

assuming a linear diffusion path. $m$ is the mass of a hydrogen atom and $k$ is the curvature of the energy plot at the base of the energy basin as shown in Fig. 3.4.

The VASP package used for these simulations, which was built with the customization from the Henkelman group for climbing image NEB method [46] allows us to find the exact peak of the energy landscape. Super cell size, K-point mesh density, and the plane wave cut-off energy was kept the same as for the original DFT calculations. Each energy landscape consists of nine images between the two fixed endpoints. The density of the images is higher closer to the end points to increase the accuracy of the curvature calculation.

\subsubsection{Kinetic Monte Carlo Simulation}

KMC simulations were carried out using an in-house code which uses the hopping rates and displacements of each transition step as particles diffuse. For each simulation we considered the diffusion of a single particle in a medium which agrees well with the Einstein-Smoluchowski relation where no driving forces are present. Table 3.2, Fig. 3.5, and Fig. 3.6 show the different paths available for the diffusion of hydrogen in the hcp zirconium lattice through its occupancy locations. We introduced periodic boundary conditions to the KMC simulations, where if the hydrogen atom leaves the periodic cell (for all our simulations, we use a single hcp unit cell of zirconium as the 
Table 3.2: Paths available for hydrogen to escape from an occupancy location considering its neighboring interstitial sites in the hcp Zr crystal structure

\begin{tabular}{cccc}
\hline Location of H atom & Neighbor site & $\begin{array}{c}\text { Number of neighbor } \\
\text { site }\end{array}$ & $\begin{array}{c}\text { Transition } \\
\text { reference }\end{array}$ \\
\hline \hline \multirow{3}{*}{ Tetrahedral Site } & NN Tetrahedral & 1 & $\mathrm{~T}-\mathrm{T}$ \\
& NN Octehedral & 3 & $\mathrm{~T}-\mathrm{O}$ \\
& 2nd NN Tetrahedral & 3 & $\mathrm{~T}-2 \mathrm{~T}$ \\
\hline \multirow{2}{*}{ Octahedral Site } & NN Tetrahedral & 6 & $\mathrm{O}-\mathrm{T}$ \\
& NN Octehedral & 2 & $\mathrm{O}-\mathrm{O}$ \\
\hline
\end{tabular}

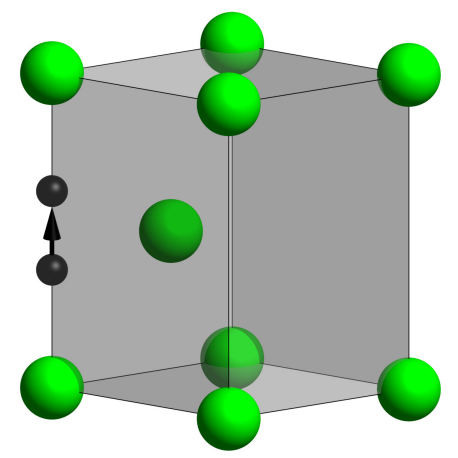

(a) NN tetrahedral site

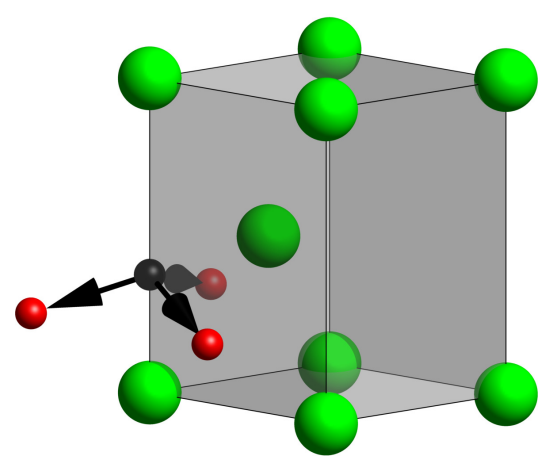

(b) NN octahedral site

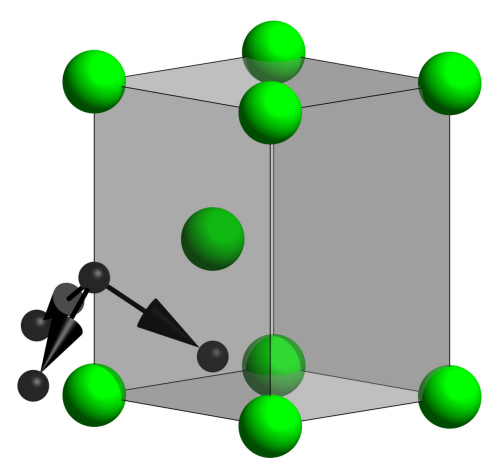

(c) 2nd NN tetrahedral sites

Figure 3.5: Arrows show the escape paths available for a hydrogen atom occupying a tetrahedral interstitial site

periodic cell) it is mapped back to the respective location to simulate the effect of a continuum. This approach allows KMC to be carried out for the smallest possible repeating unit cell which will reduce the effort required in developing input data for the simulations.

We carried out $\mathrm{KMC}$ for temperatures from $100^{\circ} \mathrm{C}-300^{\circ} \mathrm{C}$ in steps of $50^{\circ} \mathrm{C}$. For each temperature we ran $2500 \mathrm{KMC}$ simulations with different initial configurations (occupancy locations and random number seeds) with each calculation simulating the movement of a single hydrogen atom. These were run for a time, which would allow approximately 1000000 diffusion steps. At each step the total displacement, the 


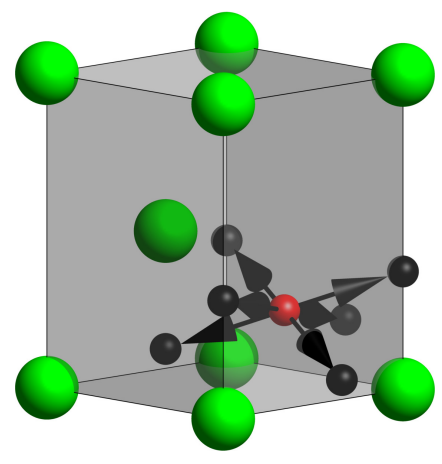

(a) NN tetrahedral site

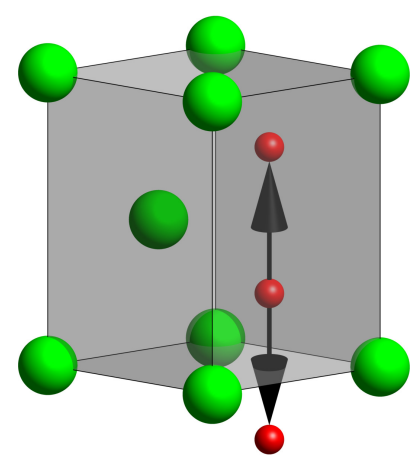

(b) NN octahedral sites

Figure 3.6: Arrows show the escape paths available for a hydrogen atom occupying a octahedral interstitial site

elapsed time, and sum of the squares of individual diffusion steps were updated until the total time reaches the limiting value.

Using KMC, diffusivity of hydrogen was calculated for different temperatures in the $X, Y$, and $Z$ directions, which was then used to determine $D_{0}$ and $E_{a}$ for bulk diffusion in the respective directions, by fitting the $D$ vs $T$ results to eqn. (3.6).

\subsubsection{Pseudo Energy Basin Effect}

During the KMC process it was noticed that the transition between the pairs of nearest neighbor tetrahedral sites has a very high rate constant (due to the low activation barrier) compared to the remaining transitions. This caused the hydrogen atom to oscillate between these sites for a vast majority of the diffusion steps. This does not contribute to the bulk diffusion, acting like a single energy basin or interstitial site (a pseudo-basin). As such, eqn. (3.5) will not provide accurate results, and will greatly overestimate the diffusivity in the axis direction by several orders (the diffusivity of axis direction is about 1250 times the diffusivity in basal plane direction at $100^{\circ} \mathrm{C}$ and 90 times at $250^{\circ} \mathrm{C}$ ) and increase the number of diffusion steps required by $\mathrm{KMC}$ for convergence. In this case, we can either rely exclusively on eqn. (3.3).

In situations where one might want to run many simulations on similar systems, the slow convergence of eqn. (3.3) may make it worth the effort to correct the behaviour of eqn. (3.5) using pseudo-basins. We have done this here in the case of the nearest neighbor tetrahedral sites. To illustrate the problem and solution, we present details of one KMC simulation $300^{\circ} \mathrm{C}$. The results from eqn. (3.3) and eqn. (3.5) 
Table 3.3: Comparison of diffusivity with KMC simulations for the modified pseudo energy basin system and the unmodified system

\begin{tabular}{cccc}
\hline Diffusivity Value & \multicolumn{2}{c}{ Unmodified } & Modified (from eqn. (3.5)) \\
\cline { 2 - 3 }$\left(\times 10^{-7} \mathrm{~cm}^{2} / \mathrm{s}\right)$ & from eqn. (3.3) & from eqn. (3.5) & \\
\hline \hline$X / Y$-direction & 5.874 & 5.897 & 5.892 \\
$Z$-direction & 6.697 & 299.83 & 6.684 \\
\hline
\end{tabular}

are shown in the column labelled "Unmodified" in Table 3.3. While the results agree in the $X$ and $Y$ directions, there is a clear discrepancy along $Z$-direction, where eqn. (3.5) fails in this case because the low energy barrier between nearest neighbor tetrahedral sites invalidates its assumptions. and at $300^{\circ} \mathrm{C}$ with the unmodified rate constants (i.e., without employing the pseudo-basin) at $300^{\circ} \mathrm{C}$ temperature and evaluated the diffusivity values for each direction from both equations eqn. (3.3) and eqn. (3.5) in the paper. Comparison of these values with the diffusivity from the modified method are as shown in Table 3.3 below. From this we can see that even without the modification for the pseudo-basin we could still determine the diffusivity values using this method. The advantage of the modification lies in the efficiency where we can achieve results faster in the KMC simulations once the modification was done. The modification allows us to simulate longer time intervals using a smaller number of steps.

To solve this, we carried out many KMC simulations to determine the exit time from this pseudo-basin and modified the diffusivity parameters for the remaining transition hops from the pseudo-basin/interstitial site. Results of these calculations are shown in Sec. 3.5.2. Thereafter, regular KMC was carried out where the nearest neighbor tetrahedral sites are combined to form a single occupancy site as shown in Fig. 3.7. With this modification, the escape paths shown in Table 3.2 and Fig. 3.5 are changed to Table 3.4 and Fig. 3.8. The number of tetrahedral sites (combined sites) per unit cell reduced to two. This is similar to the approach of Zhang et al. [15] used in his studies.

In the column of Table 3.3 labelled "Modified", we show that these modifications 


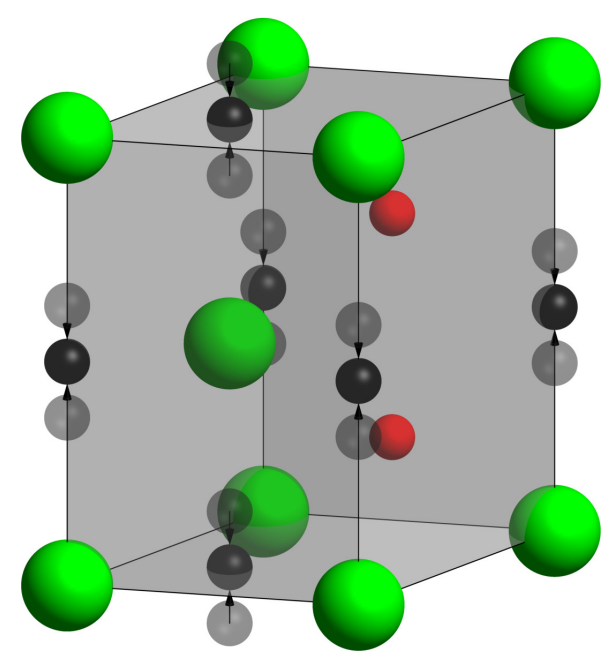

Figure 3.7: Modified interstitial site arrangement is shown here. Black color spheres shows the locations of the interstitial site representing the pseudo-basin and the light grey spheres shows the tetrahedral interstitial sites which combined to form these sites

Table 3.4: Paths available for hydrogen to escape from an occupancy location considering its neighboring interstitial sites for the modified occupancy locations

\begin{tabular}{cccc}
\hline Location of H atom & Neighbor site & $\begin{array}{c}\text { Number of neighbor } \\
\text { site }\end{array}$ & $\begin{array}{c}\text { Transition } \\
\text { reference }\end{array}$ \\
\hline \hline \multirow{2}{*}{ Tetrahedral Site } & NN Octehedral & 6 & T-O \\
& 2nd NN Tetrahedral & 6 & T-2T \\
\hline \multirow{2}{*}{ Octahedral Site } & NN Tetrahedral & 6 & O-T \\
& NN Octehedral & 2 & O-O \\
\hline
\end{tabular}




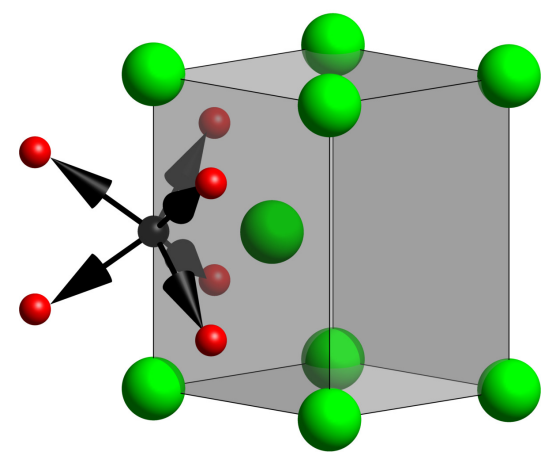

(a) NN octahedral site

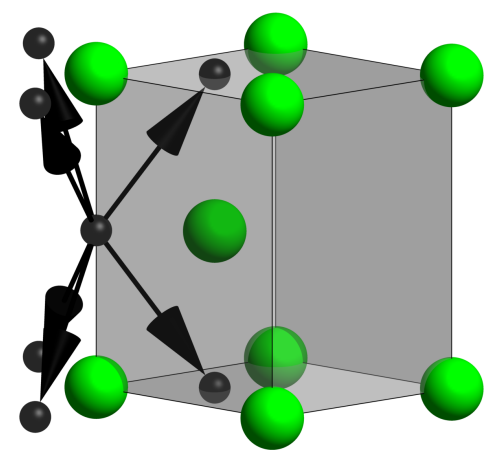

(b) 2nd $\mathrm{NN}$ tetrahedral sites

Figure 3.8: Arrows show the escape paths available for a hydrogen atom occupying a modified tetrahedral interstitial site

indeed bring eqn. (3.5) back into agreement with eqn. (3.3).

\subsection{Results and Discussion}

\subsubsection{Rate Constants}

The rate constants for the different diffusion steps were evaluated using NEB and DFT as per the conditions mentioned in Sec. 3.4. Fig. 3.9 shows the energy landscapes for each of the different transition steps available relative to the energy of the starting point, plotted against a normalized reaction coordinate. This shows that the transition between two tetrahedral sites happens with very low energy barrier compared to the rest of the transitions. Hence it would happen with a very high probability at moderate and low temperatures. The transition to the 2nd nearest neighbor tetrahedral site would be a rare occurrence due to the much higher activation energy, hence it could even be neglected during the study without any significant effect.

From the procedure mentioned in the Sec. 3.4.1, we determined the components of the rate parameters as shown in Table 3.5. Comparing the activation barriers $E_{a}$, with results from the literature, we see a good agreement. The transitions from the octahedral sites show a slightly higher values where the differences are similar to the higher stability of the octahedral sites found in the current study. These differences could be attributed to the temperature effects suggested by Christensen et al. [10] 


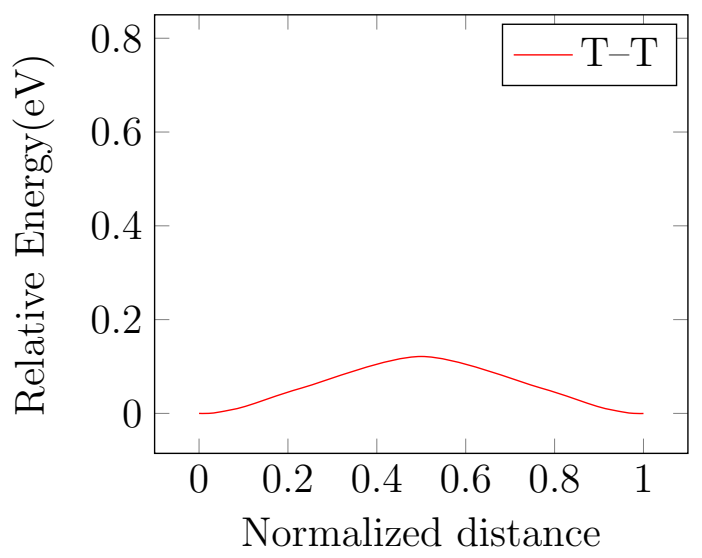

(a) $\mathrm{T}-\mathrm{T}$

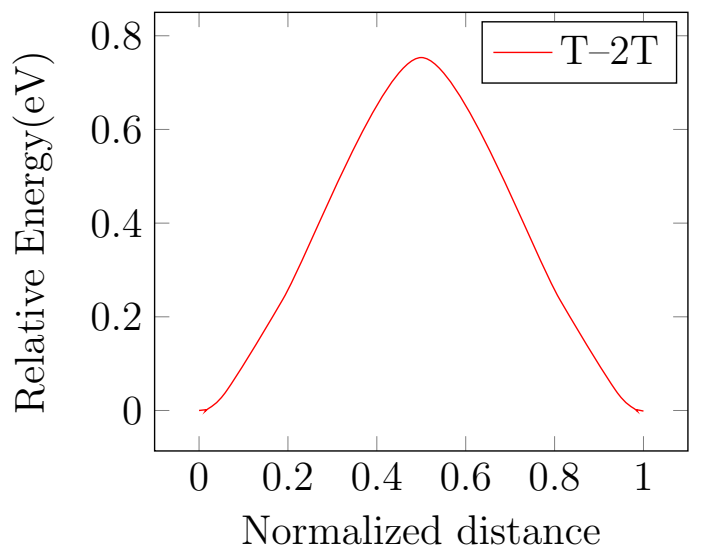

(c) $\mathrm{T}-2 \mathrm{~T}$

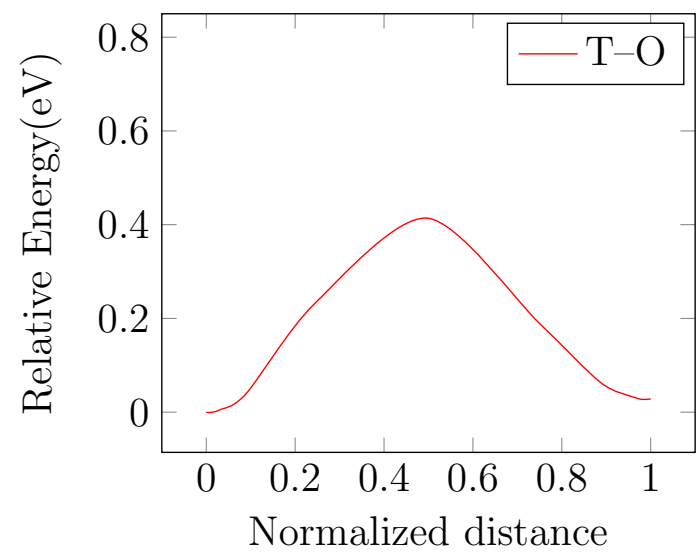

(b) $\mathrm{T}-\mathrm{O}$

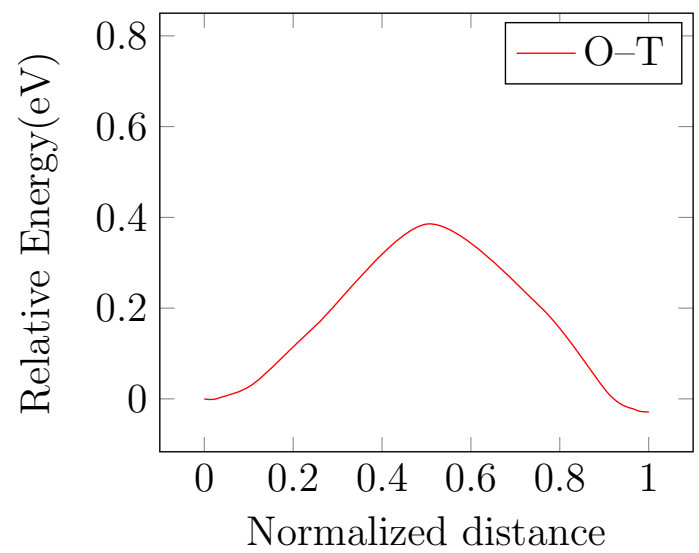

(d) $\mathrm{O}-\mathrm{T}$

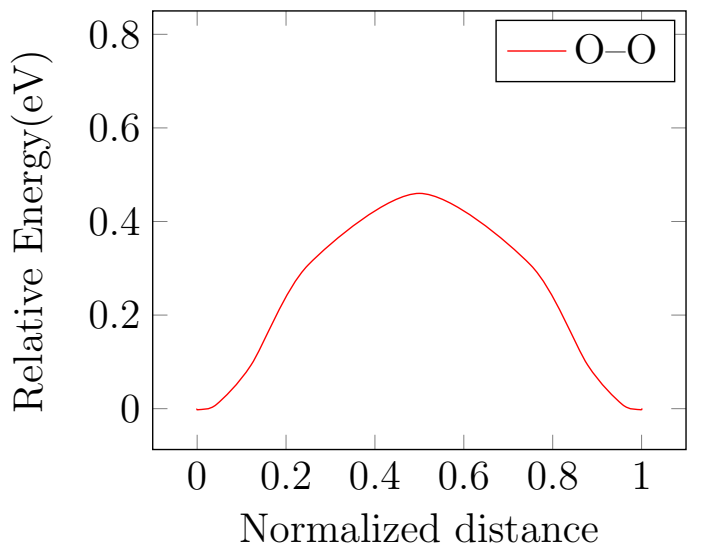

(e) $\mathrm{O}-\mathrm{O}$

Figure 3.9: Energy landscapes for different diffusion steps in Table 3.2 obtained through first principles calculations 
Table 3.5: $\Gamma_{0}$ and $E_{a}$ for different diffusion steps in Table 3.2 obtained through first principles calculations and $E_{a}$ from previous work are included for comparison

\begin{tabular}{cccccc}
\hline \multirow{2}{*}{ Diffusion Step } & \multicolumn{4}{c}{$E_{a}(\mathrm{eV})$} & $\Gamma_{0}\left(10^{13} \mathrm{~s}^{-1}\right)$ \\
\cline { 2 - 5 } & $\begin{array}{c}\text { Zhang } \\
\text { et al. [15] }\end{array}$ & $\begin{array}{c}\text { Christensen } \\
\text { et al. [19] }\end{array}$ & $\begin{array}{c}\text { Domain } \\
\text { et al. [22] }\end{array}$ & $\begin{array}{c}\text { Current } \\
\text { Study }\end{array}$ & $\begin{array}{c}\text { Current } \\
\text { Study }\end{array}$ \\
\hline \hline T-T & 0.129 & 0.129 & 0.120 & 0.121 & 4.986 \\
T-O & 0.406 & 0.412 & 0.410 & 0.414 & 5.798 \\
T-2T & - & - & - & 0.753 & 4.938 \\
O-T & 0.346 & - & 0.350 & 0.387 & 3.588 \\
O-O & 0.398 & 0.427 & 0.410 & 0.460 & 4.308 \\
\hline
\end{tabular}

and the stress-free diffusion allowed in the current study.

\subsubsection{Modification for the Pseudo-Basin}

Through KMC simulations, we calculated the average time it would take to exit a pseudo-basin through $\mathrm{T}-\mathrm{O}$ and $\mathrm{T}-2 \mathrm{~T}$ transitions at different temperatures. The modified rate constant $\Gamma$ is obtained as the reciprocal of the mean basin exit time. The plot of the modified rate constant with the temperature is shown in Fig. 3.10. This shows that the modified rates still follow an Arrhenius form. We obtained the modified parameters $\Gamma_{0}$ and $E_{a}$ shown in Table 3.6 by curve fitting. Comparing this with respective values in Table 3.5 shows that the change happens mainly to the $\Gamma_{0}$ value which shows a reduction, more significantly in the $\mathrm{T}-\mathrm{O}$ diffusion step. During the simulation it was noted that $\mathrm{T}-2 \mathrm{~T}$ happens very rarely with a probability less than $0.15 \%$ in the considered temperature range, as expected with the high activation barrier for this diffusion step. The modified parameters of Table 3.6 were used for the determination of the bulk diffusivity of zirconium. 


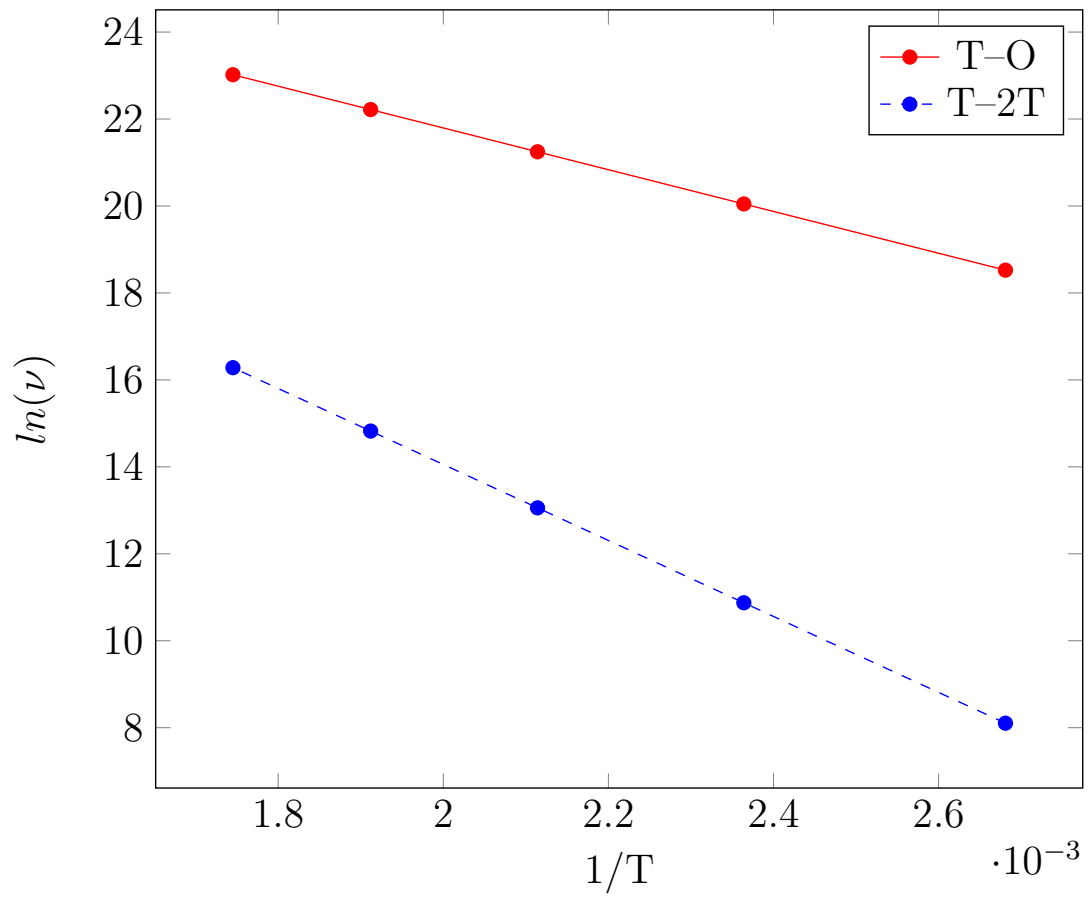

Figure 3.10: Plot of the modified rate constants vs the temperature

Table 3.6: Modified $\Gamma_{0}$ and $E_{a}$ for diffusion steps obtained through KMC simulations

\begin{tabular}{ccc}
\hline Diffusion step & $\Gamma_{0}\left(10^{13} s^{-1}\right)$ & $E_{a}(e V)$ \\
\hline \hline $\mathrm{T}-\mathrm{O}$ & 4.347 & 0.414 \\
$\mathrm{~T}-2 \mathrm{~T}$ & 4.935 & 0.753 \\
\hline
\end{tabular}




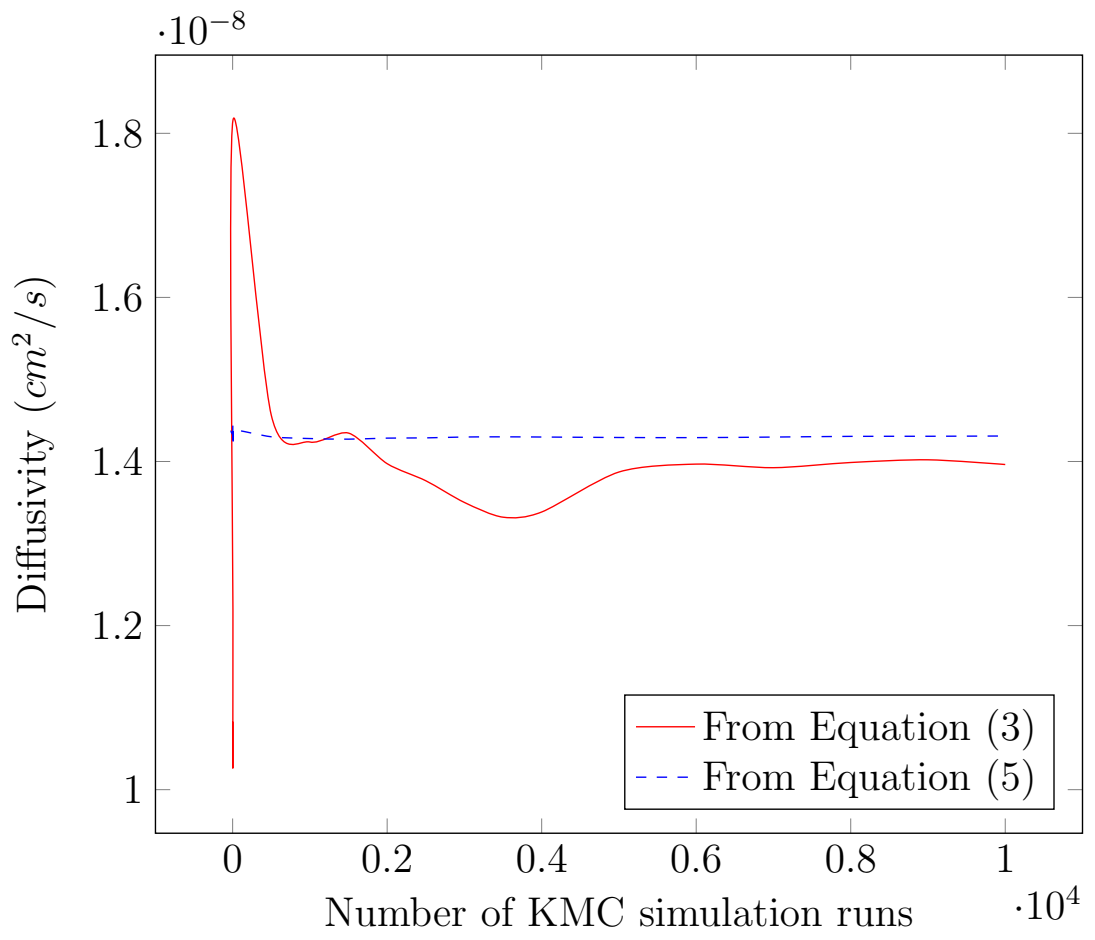

Figure 3.11: Diffusivity calculated from eqn. (3.3) and eqn. (3.5) for basal plane direction at $100^{\circ} \mathrm{C}$ for different number of simulation runs

\subsubsection{Diffusivity of Hydrogen in Zirconium}

$\mathrm{KMC}$ simulations were carried for different temperatures to estimate the mean square displacement of particles for different temperatures. We checked the convergence of the $\mathrm{KMC}$ simulation for 10000 simulation runs at $100^{\circ} \mathrm{C}$. Fig. 3.11 shows the variation of the diffusivity in basal plane direction calculated through eqn. (3.3) and eqn. (3.5) for different number of simulation runs. It shows that the use of the sum of individual diffusion steps eqn. (3.5) is much more efficient as it converges much faster than using total displacements eqn. (3.3)) which would save a significant amount of time and resources.

Diffusivity of hydrogen in hep zirconium was determined from KMC simulations for the temperature range $100-300^{\circ} \mathrm{C}$ to simulate the operational conditions in nuclear reactors. eqn. (3.12) shows the bulk diffusivity of hydrogen in zirconium in Cartesian coordinate directions, where $X$ and $Y$ directions are in the basal plane ( $A B$ plane in Fig. 3.2(b)) and $Z$-direction is in the $C$-axis direction. Curves for the $X$ and $Y$ directions overlap each other exactly, which is expected as the diffusion in basal 


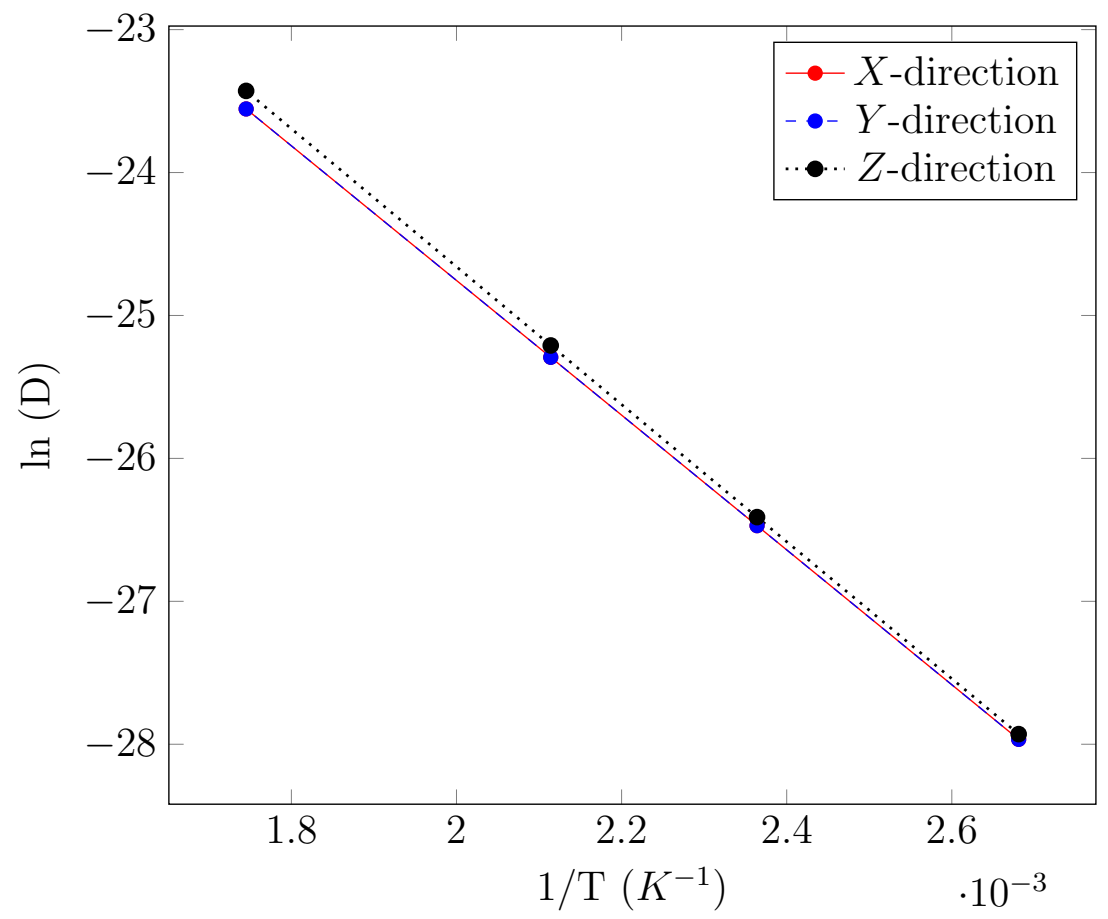

Figure 3.12: Bulk Diffusivity vs temperature computed from KMC simulations and eqn. (3.5)

Table 3.7: $D_{0}$ and $E_{a}$ for the bulk diffusion obtained through curve fitting of Fig. 3.12

\begin{tabular}{ccc}
\hline Diffusivity Parameter & $\mathrm{x} / \mathrm{y}$-direction & z-direction \\
\hline \hline$D_{0}\left(\mathrm{~cm}^{2} / \mathrm{s}\right)$ & $2.20 \times 10^{-3}$ & $2.94 \times 10^{-3}$ \\
$E_{a}(\mathrm{eV})$ & 0.406 & 0.414 \\
\hline
\end{tabular}

plane is isotropic. The diffusion in the $Z$-direction is quite close to the basal plane direction, which goes well with the hypothesis of McRae et al. [24] and Christensen et al. [10] who claim that bulk diffusivity of hydrogen in zirconium is isotropic. $D_{0}$ and $E_{a}$ for the basal plane and axis direction are presented in Table 3.7 and show that the variation of the two directions is minimal. These parameters are in the same order values from previous literature shown in Table 3.1. The agreement is much closer to the previous computational studies, which could mean that the variation with the experimental studies may be due to environmental factors, such as effects of stress.

Let's define anisotropy as $D_{C} / D_{A B}$ where $D_{C}$ is the diffusivity in axis direction 


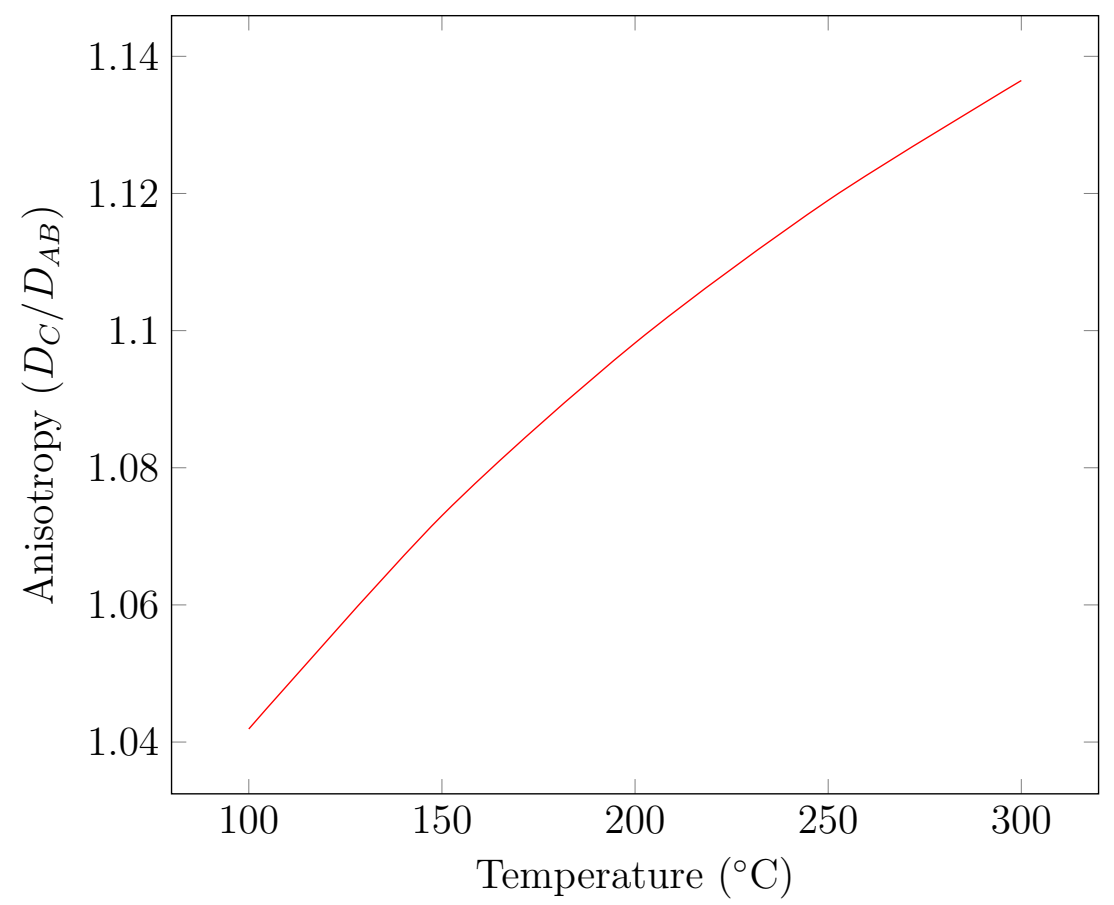

Figure 3.13: Anisotropy of bulk diffusion as defined in eqn. (3.8) for reactor operating temperatures

and $D_{A B}$ is the diffusivity in basal plane directions shown in Fig. 3.2(b). From Fig. 3.13 we can see that the anisotropy of the diffusion in the considered range is quite small for reactor temperature $\left(100-300^{\circ} \mathrm{C}\right)$. With higher temperatures the diffusion in the axis direction increases with respect to the basal plane directions with the change decreasing at higher temperatures. Since both $D_{C}$ and $D_{A B}$ follows the form of eqn. (2.34) anisotropy $D_{C} / D_{A B}$ is simply,

$$
\frac{D_{C}}{D_{A B}}=1.336 \exp \left(\frac{-0.008}{k_{\mathrm{B}} T}\right)
$$

which shows that the maximum value for anisotropy is around 1.34 which occurs at very high temperatures.

To find the reason for this nearly isotropic behavior, we looked at the individual diffusion steps which mainly contribute to the bulk diffusion. Comparing the probability of paths taken for escape from the interstitial sites (see Table 3.8) we can see that the transitions between tetrahedral and octahedral sites $(\mathrm{T}-\mathrm{O}$ and $\mathrm{O}-\mathrm{T})$ are mainly responsible for the bulk diffusion and the transition between the octahedral 
Table 3.8: Probability of selecting an escape path from each occupancy location for the modified interstitial arrangement

\begin{tabular}{lcccc}
\hline \multirow{2}{*}{ Occupancy Site } & Escape Path & \multicolumn{3}{c}{ Probability } \\
\cline { 3 - 5 } & & $100^{\circ} \mathrm{C}$ & $200^{\circ} \mathrm{C}$ & $300^{\circ} \mathrm{C}$ \\
\hline \hline \multirow{2}{*}{ Tetrahedral site } & $\mathrm{T}-\mathrm{O}$ & $100.00 \%$ & $99.97 \%$ & $99.88 \%$ \\
& $\mathrm{~T}-2 \mathrm{nd} N \mathrm{~N}$ T & $0.00 \%$ & $0.03 \%$ & $0.12 \%$ \\
\hline \multirow{2}{*}{ Octahedral site } & $\mathrm{O}-\mathrm{T}$ & $88.98 \%$ & $83.31 \%$ & $78.51 \%$ \\
& $\mathrm{O}-\mathrm{O}$ & $11.02 \%$ & $16.69 \%$ & $21.49 \%$ \\
\hline
\end{tabular}

sites $(\mathrm{O}-\mathrm{O})$ has a smaller contribution which increases with temperature. The mean square displacement values of the transitions between the tetrahedral and the octahedral sites are equal to $1.741 \AA^{2}$ and $1.667 \AA^{2}$ in basal plane and axis directions respectively. Diffusivity is proportional to mean square displacement values in these respective directions if the bulk diffusion only comprises of $\mathrm{T}-\mathrm{O}$ and $\mathrm{O}-\mathrm{T}$ transitions. This would mean that the diffusivity of hydrogen in zirconium needs to be close to isotropic, as the mean square displacement values are close to each other with only a slightly higher value in the basal plane directions. The contribution from the $\mathrm{O}-\mathrm{O}$ transition increases the mean square displacement in the axis direction, which in turn increases the relative diffusivity in axis direction. At higher temperatures the contribution from this type of step is higher, which explains the increase in anisotropy $\left(D_{C} / D_{A B}\right)$ with temperature.

\subsubsection{Modelling Process}

The proposed method has potential application in modelling other diffusion processes which comprise clearly distinguishable diffusion steps to determine their diffusivity. Interstitial diffusion and vacancy diffusion in other crystal structures are examples for diffusion processes which could be simulated with the proposed method. We present a process graph which describes the modelling process in detail in Fig. 3.14. 


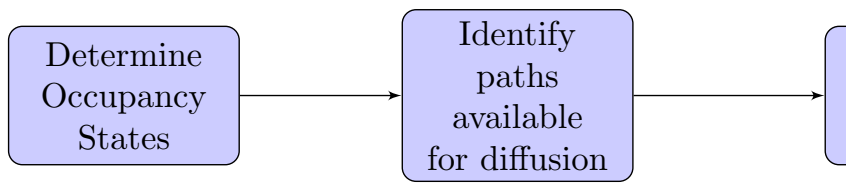

Evaluate energy landscapes for diffusion paths

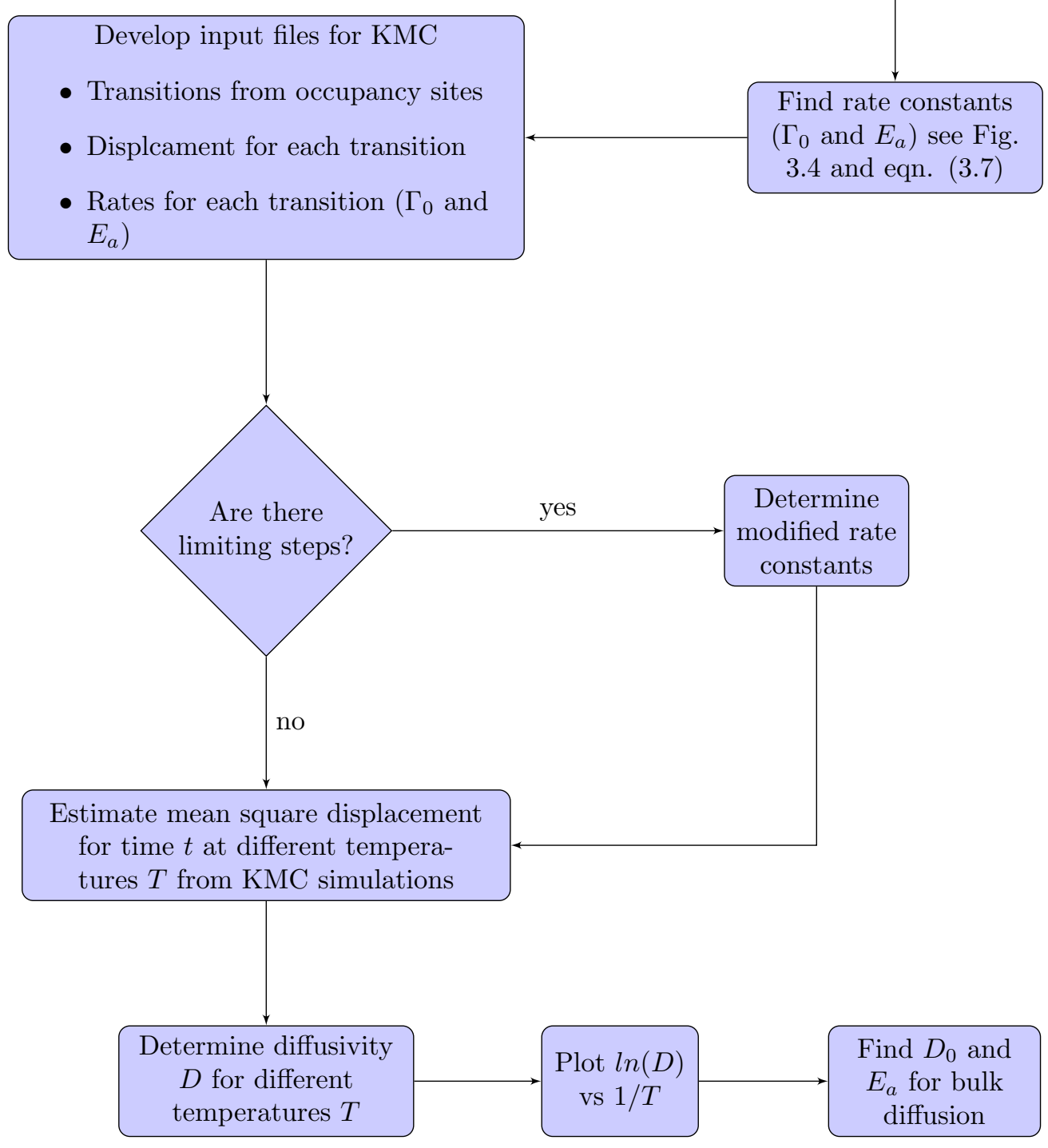

Figure 3.14: Modelling process followed in the current study 


\subsubsection{Further Discussion}

In this study, we have neglected zero-point energy (ZPE) effects. Several studies in hydrogen diffusion has found that the activation energy will decrease when ZPE is considered which would result in higher diffusivity values [47-50]. McIntosh et al. [49] has found a reduction of about $0.030 \mathrm{eV}$ in the activation barrier when ZPE is considered. One way to address this would be to find the correction for the activation barrier as the difference between the ZPE at the saddle point and the ZPE of the initial position [51]. We will examine these effects in a future study.

In our work, all DFT calculations are performed at zero temperature. In KMC simulations, we have assumed that the temperature effect on rate constant will be significant compared to other effects such as anisotropic thermal expansion. In zirconium, the thermal expansion coefficients in a and c directions are $5.5 \times 10^{-6} \mathrm{~K}^{-1}$ and $10.8 \times 10^{-6} K^{-1}$ respectively [52]. Hence, we can expect small changes to the lattice parameters and anisotropy at the temperature range we used for our study. Lattice parameters a and c will increase by $0.2 \%$ and $0.4 \%$ at $100{ }^{\circ} \mathrm{C}$ and $0.3 \%$ and $0.6 \%$ at $300{ }^{\circ} \mathrm{C}$. Finite temperature DFT calculations could improve the accuracy of our calculations, but they are computationally very expensive [53-55].

Our study focusses on a single crystal, allowing us to establish the intrinsic diffusion characteristics of the hcp lattice. In applications, $\mathrm{Zr}$ is of course almost always polycrystalline. Grain boundaries and other defects will also play a role on the diffusion of $\mathrm{H}$, and the relative size of these contributions needs to be determined and perhaps taken into account. This could be explored by incorporating the behaviour of hydrogen in grain boundaries in larger simulations, although the complex and varied nature of grain boundaries mean that this would require extensive investigation beyond the scope of this work. However, any of these configurations could, in principle, be studied using the methods we have described herein. Similarly, this approach can be used for other microstructures in other material systems so long as the computational resources are available.

\subsection{Conclusion and Future Directions}

We describe a comprehensive method to determine the bulk diffusivity of hydrogen in zirconium, using a combination of first principles methods and $\mathrm{KMC}$ simulation. The first principles calculations have been successfully used to determine the rate 
constants for individual diffusion steps, giving values which match well with previous work. This study concludes that hydrogen diffusion in zirconium is nearly isotropic, with more anisotropy with increasing temperature. We used the results from first principles calculations to identify the reasons for the isotropic nature of hydrogen diffusion in zirconium even though hcp zirconium has an anisotropic crystal structure.

The approach used in this paper to determine the diffusivity of a particle in a certain medium can be used to study diffusion processes consisting of multiple, clearly understood diffusion steps. We have interest in studying diffusion of hydrogen related to the formation of hydride denuded zones in zircaloy pressure vessels. Lead zirconate titanate is another material which has been affected by hydrogen degradation and a topic under investigation. The current study provides a verification that the proposed method can be extended to these other studies. Another interest is to look at how stress will affect the diffusion of zirconium, which may be used to check the validity of the hypothesis of McRae et al. [24] and could explain the discrepancies between previous hydrogen diffusion studies.

\section{Bibliography}

[1] Y. Wang, W. Y. Chu, L. J. Qiao, and Y. J. Su, "Hydrogen-induced delayed fracture of PZT ceramics during dynamic charging under constant load," Materials Science and Engineering, vol. 98, no. 1, pp. 1-5, 2003.

[2] X. Peng, Y. J. Su, K. W. Gao, L. J. Qiao, and W. Y. Chu, "Hydrogen fissure in PZT ferroelectric ceramic," Materials Letters, vol. 58, no. 15, pp. 2073-2075, 2004.

[3] G. Lu and E. Kaxiras, "Hydrogen embrittlement of aluminum: The crucial role of vacancies," Physical Review Letters, vol. 94, no. 15, pp. 155501 1-5, 2005.

[4] J. Song and W. A. Curtin, "A nanoscale mechanism of hydrogen embrittlement in metals," Acta Materialia, vol. 59, p. 1557-1569, 2011.

[5] K. J. Alvine, M. Vijayakumar, M. E. Bowden, A. L. Schemer-Kohrn, and S. G. Pitman, "Hydrogen diffusion in lead zirconate titanate and barium titanate," Journal of Applied Physics, vol. 112, no. 4, pp. 043511 1-6, 2012.

[6] J. Song and W. A. Curtin, "Atomic mechanism and prediction of hydrogen embrittlement in iron.," Nature materials, vol. 12, no. 2, pp. 145-151, 2013.

[7] M. S. Blackmur, J. D. Robson, M. Preuss, O. Zanellato, R. J. Cernik, S. Q. Shi, F. Ribeiro, and J. Andrieux, "Zirconium hydride precipitation kinetics in zircaloy-4 observed with synchrotron x-ray diffraction," Journal of Nuclear Materials, vol. 464, p. 160-169, 2015. 
[8] E. Chajduk and A. Bojanowska-Czajka, "Corrosion mitigation in coolant systems in nuclear power plants," Progress in Nuclear Energy, vol. 88, pp. 1-9, 2016.

[9] International Atomic Energy Agency, Corrosion of zirconium alloys in nuclear power plants. Vienna, Austria: International Atomic Energy Agency, 1993.

[10] M. Christensen, W. Wolf, C. Freeman, E. Wimmer, R. B. Adamson, L. Hallstadius, P. Cantonwine, and E. V. Mader, "Effect of hydrogen on dimensional changes of zirconium and the influence of alloying elements: First-principles and classical simulations of point defects, dislocation loops, and hydrides," in STP1543-EB Zirconium in the Nuclear Industry (B. Comstock and P. Barberis, eds.), vol. 17, pp. 55-92, West Conshohocken, PA: ASTM International, 2015.

[11] Y. Gou, Y. Li, Y. Liu, H. Chen, and S. Ying, "Evaluation of a delayed hydride cracking in zr-2.5nb candu and rbmk pressure tubes," Materials and Design, vol. 30, no. 4, pp. 1231-1235, 2009.

[12] International Atomic Energy Agency, Delayed hydride cracking in zirconium alloys in pressure tube nuclear reactors. Vienna: International Atomic Energy Agency, 2004.

[13] R. N. Singh, T. K. Sinha, and B. P. Kashyap, "Delayed hydride cracking in Zr-2 . 5Nb pressure tube material," Journal of Nuclear Materials, vol. 304, pp. 189-203, 2002.

[14] S. Suman, M. K. Khan, M. Pathak, R. N. Singh, and J. K. Chakravartty, "Hydrogen in Zircaloy: Mechanism and its impacts," International Journal of Hydrogen Energy, vol. 40, pp. 5976-5994, 2015.

[15] Y. Zhang, C. Jiang, and X. Bai, "Anisotropic hydrogen diffusion in $\alpha$-Zr and Zircaloy predicted by accelerated kinetic Monte Carlo simulations," Scientific Reports, vol. 7, pp. 41033 1-13, 2017.

[16] N. A. P. K. Kumar, Hydride formation in zirconium alloys. PhD thesis, McGill University, 2011.

[17] M. Ma, W. Xiang, B. Tang, L. Liang, L. Wang, and X. Tan, "Non-isothermal and isothermal hydrogen desorption kinetics of zirconium hydride," Journal of Nuclear Materials, vol. 467, pp. 349-356, 2015.

[18] P. A. Burr, S. T. Murphy, S. C. Lumley, M. R. Wenman, and R. W. Grimes, "Hydrogen accommodation in zr second phase particles: implications for h pickup and hydriding of zircaloy-2 and zircaloy-4," Corrosion Science, vol. 69, pp. 14, 2013.

[19] M. Christensen, W. Wolf, C. Freeman, E. Wimmer, R. B. Adamson, L. Hallstadius, P. E. Cantonwine, and E. V. Mader, " $\mathrm{H}$ in $\alpha$-zr and in zirconium hydrides: solubility, effect on dimensional changes, and the role of defects," Journal of Physics: Condensed Matter, vol. 27, pp. 025402 1-12, 2015. 
[20] S. C. Lumley, R. W. Grimes, S. T. Murphy, P. A. Burr, A. Chroneos, P. R. ChardTuckey, and M. R. Wenman, "The thermodynamics of hydride precipitation: The importance of entropy, enthalpy and disorder," Acta Materialia, vol. 79, pp. 351$362,2014$.

[21] L. A. Simpson and C. D. Cann, "Fracture toughness of zirconium hydride and its influence on the crack resistance of zirconium alloys," Journal of Nuclear Materials, vol. 87, pp. 303-316, 1979.

[22] C. Domain, R. Besson, and A. Legris, "Atomic-scale ab-initio study of the zrh system: I. bulk properties," Acta Materialia, vol. 50, no. 13, pp. 3513-3526, 2002 .

[23] J. J. Kearns, "Diffusion coefficient of hydrogen in alpha zirconium, zircaloy-2 and zircaloy-4," Journal of Nuclear Materials, vol. 43, no. 3, pp. 330-338, 1972.

[24] G. McRae, C. Coleman, and H. Nordin, "Diffusivity of hydrogen isotopes in zirconium alloys interpreted with the einstein flux equation," 2017. Presentation in the Canadian Material Science Conference, Ottawa ON.

[25] M. Someno, "Solubility and diffusion of hydrogen in zirconium," Nippon Kinzoku Gakkaishi (Japan), vol. 24, pp. 249-253, 1960.

[26] M. W. Mallett and W. M. Albrecht, "Low-pressure solubility and diffusion of hydrogen in zirconium," Journal of The Electrochemical Society, vol. 104, no. 3, pp. 142-146, 1957.

[27] S. Naito, "Kinetics of hydrogen absorption by $\alpha$-zirconium," The Journal of Chemical Physics, vol. 79, no. 6, pp. 3113-3120, 1983.

[28] F. Mazzolai and J. Ryll-Nardzewsi, "An anelastic study of the diffusion coefficient of hydrogen in $\alpha$-zirconium," Journal of the Less-Common Metalls, vol. 49, pp. 323-327, 1976.

[29] A. Sawatzky, "The diffusion and solubility of hydrogen in the alpha phase of zircaloy-2," Journal of Nuclear Materials, vol. 2, no. 1, pp. 62-68, 1960.

[30] E. A. Gulbransen and K. F. Andrew, "Diffusion of hydrogen and deuteriun in high purity zirconium," Journal of the Electrochemical Society, vol. 101, no. 11, pp. 560-566, 1953.

[31] R. K. Siripurapu, B. Szpunar, and J. A. Szpunar, "Molecular Dynamics Study of Hydrogen in $\alpha$-Zirconium," International Journal of Nuclear Energy, vol. 2014, 2014.

[32] A. Einstein, "Über die von der molekularkinetischen theorie der wärme geforderte bewegung von in ruhenden flüssigkeiten suspendierten teilchen," Annalen der Physik, vol. 322, no. 8, pp. 549-560, 1905.

[33] A. Einstein, "On the movement of small particles suspended in stationary liquids required by the molecular-kinetic theory of heat - on the random motion of particles suspended in a liquid and their relationship to diffusion," in The Swiss 
Years: Writings, 1900-1909 (English translation supplement) (J. Stachel, D. C. Cassidy, J. Renn, and R. Schulmann, eds.), vol. 2 of The Collected Papers of Albert Einstein, ch. 16, pp. 130-134, Princeton, New Jersey: Princeton University Press, 1990.

[34] G. Kresse and J. Hafner, "Ab initio molecular dynamics for liquid metals," Physical Review B, vol. 47, no. 1, pp. 558-561, 1993.

[35] G. Kresse and J. Furthmüller, "Efficiency of ab-initio total energy calculations for metals and semiconductors using a plane-wave basis set," Computational Materials Science, vol. 6, no. 1, pp. 15-50, 1996.

[36] G. Kresse and J. Furthmüller, "Efficient iterative schemes for $<\mathrm{i}>$ ab initio $</ \mathrm{i}>$ total-energy calculations using a plane-wave basis set," Physical Review B, vol. 54, no. 16, pp. 11169-11186, 1996.

[37] E. S. Fisher and C. J. Renkin, "Single-crystal elastic moduli and the hcp - bcc transformation in ti, zr, and hf," Physical Review, vol. 135, no. 2A, pp. A482A494, 1964.

[38] F. Willaime, "Ab initio study of self-interstitials in hcp-Zr," Journal of Nuclear Materials, vol. 323, pp. 205-212, 2003.

[39] G. Vérité, F. Willaime, and C. C. Fu, "Anisotropy of the vacancy migration in $\mathrm{Ti}, \mathrm{Zr}$ and Hf hexagonal close-packed metals from first principles," Solid State Phenomena, vol. 129, pp. 75-81, 2007.

[40] J. P. Perdew, K. Burke, and M. Ernzerhof, "Generalized Gradient Approximation Made Simple," Physical Review Letters, vol. 77, no. 18, pp. 3865-3868, 1996.

[41] G. Mills and H. Jónsson, "Quantum and thermal effects in $\mathrm{H}_{2}$ dissociative adsorption: Evaluation of free energy barriers in multidimensional quantum systems," Physical Review Letters, vol. 72, no. 7, pp. 1124-1127, 1994.

[42] G. Mills, H. Jónsson, and G. K. Schenter, "Reversible Work Transiton State Theory: Application to Dissociative Adsorption of Hydrogen," Surface Science, vol. 324, no. 2-3, pp. 305-337, 1995.

[43] H. Jónsson, G. Mills, and K. W. Jacobsen, "Nudged elastic band method for finding minimum energy paths of transitions," in Classical and Quantum Dynamics in Condensed Phase Simulations, ch. 16, pp. 385-404, World Scienific, 1998.

[44] G. Henkelman and H. Jónsson, "Improved tangent estimate in the nudged elastic band method for finding minimum energy paths and saddle points Improved tangent estimate in the nudged elastic band method for finding minimum energy paths and saddle points," Journal of Chemical Physics, vol. 113, pp. 9978-9985, 2000 .

[45] H. Muta, Y. Etoh, Y. Ohishi, K. Kurosaki, and S. Yamanaka, "Ab initio study of hydrogen diffusion in zirconium oxide," Journal of Nuclear Science and Technology, vol. 49, no. 5, pp. 544-550, 2012. 
[46] G. Henkelman, B. P. Uberuaga, and H. Jónsson, "Climbing image nudged elastic band method for finding saddle points and minimum energy paths," Journal of Chemical Physics, vol. 113, no. 22, pp. 9901-9904, 2000.

[47] J. B. Bates, J. C. Wang, and R. A. Perkins, "Mechanisms for hydrogen diffusion in tio $2, "$ Physical Review B, vol. 19, no. 8, pp. 4130-4139, 1979.

[48] S. Fujita and A. Garcia, "Theory of hydrogen diffusion in metals. quantum isotope effects," J. Phys. Chem. Solid., vol. 52, no. 2, pp. 351-355, 1991.

[49] E. M. McIntosh, K. T. Wikfeldt, J. Ellis, A. Michaelides, and W. Allison, "Quantum effects in the diffusion of hydrogen on ru(0001)," J. Phys. Chem. Lett., vol. 4, no. 9, pp. 1565-1569, 2013.

[50] A. Tezuka, H. Wang, H. Ogawa, and T. Ikeshoji, "Potential energy surface and hopping path for hydrogen in lanis," Phys. Rev. B, vol. 81, no. 13, p. 134304, 2010.

[51] G. Henkelman, A. Arnaldsson, and H. Jónsson, "Theoretical calculations of $\mathrm{ch}_{4}$ and $\mathrm{h}_{2}$ associative desorption from ni(111): Could subsurface hydrogen play an important role?," J. Chem. Phys., vol. 124, no. 4, p. 044706, 2006.

[52] G. B. Skinner and H. L. Johnston, "Thermal expansion of zirconium between $298 \mathrm{k}$ and 1600 k," J. Chem. Phys., vol. 21, no. 8, pp. 1383-1384, 1953.

[53] T. Hickel, B. Grabowski, F. Körmann, and J. Neugebauer, "Advancing density functional theory to finite temperatures: Methods and applications in steel design," J. Phys. Condens. Matter, vol. 24, no. 5, p. 053202, 2012.

[54] N. Mermin, "Thermal properties of the inhomogeneous electron gas," Phys. Rev., vol. 137, no. 5A, pp. A1441-1443, 1965.

[55] A. Pribram-Jones, P. E. Grabowski, and K. Burke, "Thermal density functional theory: Time-dependent linear response and approximate functionals from the fluctuation-dissipation theorem," Phys. Rev. Lett., vol. 116, no. 23, p. 233001, 2016 .

[56] K. Momma and F. Izumi, "Vesta 3 for three-dimensional visualization of crystal, volumetric and morphology data," Journal of Applied Crystallography, vol. 44, pp. 1272-1276, 2011. 


\section{Chapter 4}

\section{Denuded Zones in Zirconium Pressure Vessels: Oxygen's Role Examined via Multi-scale Diffusion Model ${ }^{1}$}

In Chapter 3 we validated the initial model we developed to simulate the diffusion behavior of $\mathrm{H}$ in a lattice structure by considering $\mathrm{H}$ diffusion in $\mathrm{Zr}$. We had some shortcomings to this model, which we discussed in Sec. 3.5.5, where the effects of zero-point energy and quantum tunnelling were ignored. Also when determining the pre-exponent of the hopping rates for individual transitions we used the curvature of the energy displacement graph obtained from DFT calculations [1]. This approach only takes the vibration of $\mathrm{H}$ in the direction of diffusion, but also it is computationally very expensive as well. Hence, for this paper, we utilize the semiclassically corrected harmonic transition state theory [2] (see Appendix A for details), which allows us to calculate the hopping rates of each transition step using the fundamental vibration frequencies for $\mathrm{H}$ in the occupancy sites, while including the effects of zero-point energy and quantum tunnelling to determine the hopping rates of the transitions. We also adopt the Markov chain approach to accelerate the KMC simulations where we combine the nearest neighbor tetrahedral sites into one "pseudo" basin. The valance configuration for $\mathrm{Zr}$ is increased from $4 d^{2} 5 s^{2}$ to $4 s^{2} 4 p^{6} 4 d^{2} 5 s^{2}$ which gives increased accuracy to the DFT results. Results obtained from this improved model gives us better results matching the previous work done, while providing improvements to the

\footnotetext{
${ }^{1}$ Contains the work in Manura Liyanage, Ronald Miller, and R. K. N. D. Rajapakse, "Denuded Zones in Zirconium Pressure Vessels: Oxygen's Role Examined via Multi-scale Diffusion Model", under review in Modelling and Simulation in Materials Science and Engineering, (Submission: January 2020).
} 
computational efficiency. Thereafter, we extended our study to include the effects of interstitial $\mathrm{O}$ in $\mathrm{Zr}$, by looking at how the stability of $\mathrm{H}$ in interstitial sites near the $\mathrm{O}$ atom and the hopping rates are affected. We carried this out for three $\mathrm{O}$ concentrations by varying the $\mathrm{O}$ atom density in a simulation cell. Then we used $\mathrm{KMC}$ simulations to determine the diffusivity of $\mathrm{H}$ under these $\mathrm{O}$ concentrations. From this we saw that $\mathrm{O}$ decreases $\mathrm{H}$ diffusivity in $\mathrm{Zr}$ which validated the theory of Hardie [3]. Then we compared the expected $\mathrm{O}$ concentration profiles in $\mathrm{Zr}$ pressure tubes against the decrease in diffusivity in $\mathrm{H}$ due to dissolved $\mathrm{O}$, and looked at the effect it has on the size of the precipitates formed. From this analysis we see that while $\mathrm{O}$ slows the diffusion of $\mathrm{H}$ in $\mathrm{Zr}$, it is not sufficient to have a noticeable size difference in the precipitates formed.

\subsection{Introduction}

Zirconium alloys are used to make pressure tubes, which contain the nuclear fuel in reactors, due to low thermal neutron absorption, good mechanical properties, and good corrosion resistance [4-6]. In the harsh service conditions of nuclear reactors, these alloys react with water to form zirconium oxide, while releasing hydrogen. Some of this hydrogen enters the metal to form hydrides which adversely change properties like the fracture toughness. Delayed hydride cracking can occur if sharp flaws, cuts, and nicks suffered during operation coincide with regions under tensile stresses [79]. Hence, the hydride content in these pressure tubes are continuously monitored through scrape samples taken from their surfaces. Optical micrographs suggest that the size of hydrides close to the metal-oxide interface can be smaller than in the bulk which hinders the monitoring process $[3,10]$. While the reasons for these hydride "denuded" zones are not exactly known, it is hypothesized that oxygen slows down the diffusion of $\mathrm{H}$ causing it to form smaller intragranular hydrides instead of the usual intergranular hydrides found as needle or plate shaped precipitates [3]. After an extensive literature review, we found no studies which has investigated this hypothesis through experimental or computational means.

We conducted a multi-scale study using first-principles calculations (Density Functional Theory) and Kinetic Monte Carlo (KMC) simulations to check the validity of this hypothesis by ascertaining the diffusivity of $\mathrm{H}$ in hexagonal close packed (hcp or $\alpha$-phase) $\mathrm{Zr}$ at zero to moderate $\mathrm{O}$ concentrations. We also looked at the reasons 
for the variation in $\mathrm{H}$ diffusivity due to the presence of $\mathrm{O}$ by looking at the diffusion mechanics and diffusion paths taken by $\mathrm{H}$ atoms.

\subsection{Computational Details}

First principles calculations were carried out with the VASP code [11-13], which gives solutions to Density Functional Theory (DFT) using pseudopotentials or the projector-augmented wave (PAW) method. We used the generalized gradient approximation of Perdew, Burke, and Ernzerhof (PBE-GGA) [14] for estimating exchange correlation energies which gives the best agreement to the lattice parameters of $\alpha-\mathrm{Zr}$. Valance configurations of $4 s^{2} 4 p^{6} 4 d^{2} 5 s^{2}, 2 s^{2} 2 p^{4}$, and $1 s^{1}$ were considered for $\mathrm{Zr}$, $\mathrm{O}$, and $\mathrm{H}$ respectively. All first principles calculations were carried out in $4 \times 4 \times 4$ $\mathrm{Zr}$ supercells (thus $144 \mathrm{Zr}$ atoms), with a plane wave cutoff energy of $500 \mathrm{eV}$, and K-point density of $5 \times 5 \times 5$ which gives very good accuracy in results. Climbing Image Nudged Elastic Band (NEB) calculations [15] were carried out to identify the activation energies of these diffusion jumps and the central difference method was used to find the vibration frequencies at energy minima and transition states. Calculated activation energies and vibration frequencies were used to determine the thermally activated hopping rates, while accounting for the Zero Point Energy (ZPE) and quantum tunneling effects using the Semi-Classical Harmonic Transition State Theory (SC-HTST) $[2,16]$. Appendix A shows the procedure in determining the hopping rate using SC-HTST. Zhang et al. [17] used this approach to determine the $\mathrm{H}$ diffusivity in $\mathrm{Zr}$, and we compared our results to theirs to validate the computational method used.

KMC simulations were used to estimate the diffusivity of $\mathrm{H}$ in bulk $\mathrm{Zr}$ with different $\mathrm{O}$ concentrations. The smallest repeating cell was used for the KMC models, while periodic boundary conditions were incorporated to simulate an infinite crystal. One challenge encountered in KMC calculations was that each pair of nearest neighbour tetrahedral sites acts as a "pseudo-energy basin", due to low activation energy for hopping between them, thus consuming the bulk of the transition steps in the KMC simulations and affecting its efficiency. The mean rate method in Puchalaet al. [18], provides an analytic solution for this problem by considering pseudo-energy basins as absorbing Markov chains. This helped in finding the modified hopping rates and the mean time of escape from the transient sites, which were used in accelerated 
KMC simulations. Appendix B shows the method for this modification and validation of the method for one of the pseudo basins encountered in this study. Hopping rates for accelerated $\mathrm{KMC}$ were obtained through this method which amalgamates the tetrahedral sites in a pseudo-energy basin to one site.

Diffusivities were calculated for $\mathrm{H}$ in pure $\mathrm{Zr}$, and at $\mathrm{O}$ concentrations of $0.775 \%$ atm, $1.82 \% \mathrm{~atm}$, and $5.88 \% \mathrm{~atm}$, for the temperature range $300 \mathrm{~K}-1100 \mathrm{~K}$ in steps of $200 \mathrm{~K}$. Each of these $\mathrm{O}$ concentrations correspond to one $\mathrm{O}$ atom in $4 \times 4 \times 4$, $3 \times 3 \times 3$, and $2 \times 2 \times 2 \mathrm{Zr}$ supercells respectively. During the study it was assumed that $\mathrm{O}$ is distributed uniformly over the $\mathrm{Zr}$ lattice, to increase the efficiency of the calculations. At low $\mathrm{O}$ concentrations, this assumption will not make a significant difference since $\mathrm{O}$ atoms will be spread apart, so their effect on $\mathrm{H}$ atoms will not be accumulated. We considered that $\mathrm{O}$ only occupies octahedral interstitial sites due to its prohibitively large size for a tetrahedral site [19,20]. Due to the low solubility of $\mathrm{H}$ atoms, they were assumed to diffuse independently from each other. $\mathrm{O}$ was considered to be stationary with respect to the $\mathrm{H}$ atoms since the diffusivity of $\mathrm{O}$ is several orders smaller than that of $\mathrm{H}$. It was also assumed that the $\mathrm{Zr}$ lattice does not have any other defects such as vacancies or dislocations to isolate the effect of interstitial $\mathrm{O}$ alone.

\subsection{Results and Discussion}

We divide this section into three subsections. In the first subsection we discuss results pertaining to diffusivity of $\mathrm{H}$ in Pure $\mathrm{Zr}$, using it to validate the methodology followed by comparing with previous work. In the second subsection we discuss the results of how $\mathrm{O}$ affects the diffusivity of $\mathrm{H}$. This subsection includes first principle calculations which shows how a single and multiple $\mathrm{O}$ atoms affect the behaviour of $\mathrm{H}$ occupying interstitial sites in its vicinity and obtain the different hopping rates for these scenarios. Then we used the ascertained hopping rates in KMC simulations to determine $\mathrm{H}$ diffusivity for different $\mathrm{O}$ concentrations and discuss how $\mathrm{O}$ affects the diffusion behavior of $\mathrm{H}$. The final section looks at how the determined diffusivity values affect the size of precipitates expected in $\mathrm{Zr}$ for the various $\mathrm{O}$ concentrations. Thereafter it looks at the validity of the hypothesis that $\mathrm{O}$ slows down the diffusion rates of $\mathrm{H}$ to form the denuded zones. 


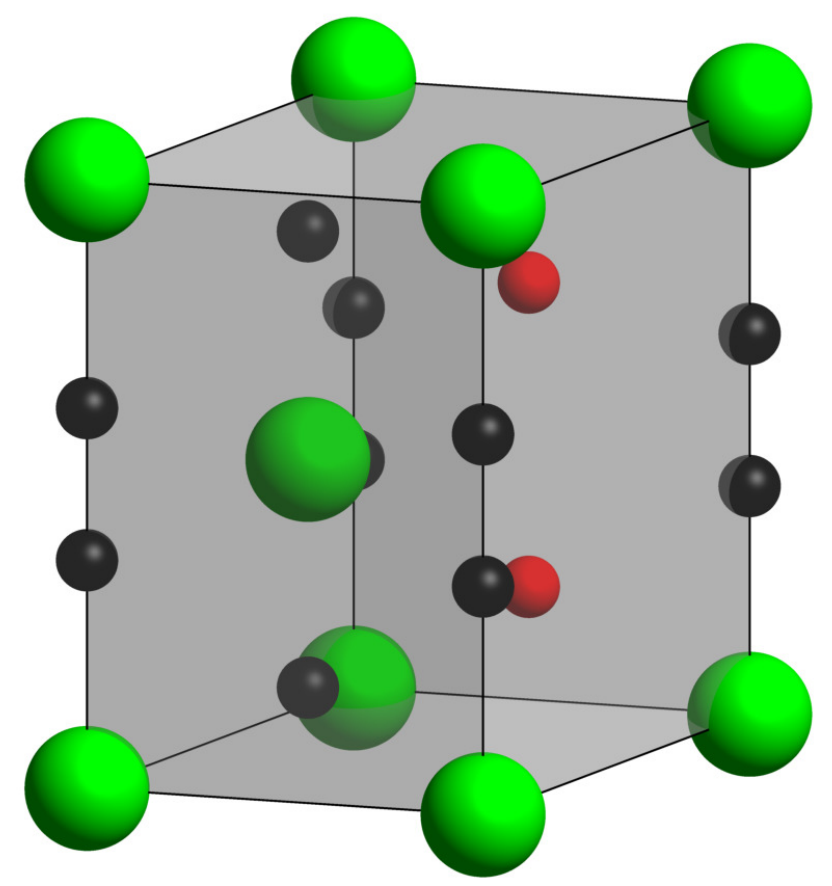

Figure 4.1: Available interstitial site locations for the occupancy of $\mathrm{H}$ in a $\mathrm{Zr}$ unit cell, where green color spheres represent the Zr atoms in the hcp lattice, while black and red color spheres represent tetrahedral and octahedral interstitial sites (respectively) available for $\mathrm{H}$ occupancy. Some of the tetrahedral sites will be shared between adjacent unit cells.

\subsubsection{Hydrogen diffusion in Pure $\mathrm{Zr}$}

Zr has a conventional unit cell as shown in Fig. 4.1, with four tetrahedral and two octahedral interstitial sites. We found that $\mathrm{H}$ favours tetrahedral sites slightly over octahedral sites, with an energetic preference of $0.067 \mathrm{eV}$. This value compares well with the values of $0.063 \mathrm{eV}, 0.057 \mathrm{eV}$, and $0.086 \mathrm{eV}$ reported in Zhang et al. [17] Domain et al. [21], and Burr et al. [22] respectively. The activation energies and the vibration frequencies for the nearest neighbour transitions obtained from NEB calculations and the central difference method, shown in Table 4.1 and Table 4.2, also show good agreement with previous work. With these activation energies and vibration frequencies, hopping rates were determined for each relevant temperature through the procedure given in Appendix A.

Using these modified rates, KMC simulations were carried out for temperatures 
Table 4.1: Comparison of activation energies of nearest neighbour transitions (in $e V)$ in $\mathrm{Zr}$ lattice with reported values. ' $\mathrm{T}$ ' denotes tetrahedral sites and ' $\mathrm{O}$ ' denotes octahedral sites.

\begin{tabular}{ccccc}
\hline Diffusion Step & Current Work & $\begin{array}{c}\text { Zhang et al. } \\
{[17]}\end{array}$ & $\begin{array}{c}\text { Domain et al. } \\
{[21]}\end{array}$ & $\begin{array}{c}\text { Christensen et al. } \\
{[23]}\end{array}$ \\
\hline \hline T-T & 0.132 & 0.129 & 0.120 & 0.129 \\
T-O & 0.408 & 0.406 & 0.410 & 0.412 \\
O-T & 0.341 & 0.346 & 0.350 & 0.360 \\
O-O & 0.403 & 0.398 & 0.410 & 0.427 \\
\hline
\end{tabular}

Table 4.2: Vibration frequencies of the initial and the transition states of each diffusion step. $\nu_{i}$ are the vibration frequencies at the initial state, $\nu_{j}^{T S}$ are the real-valued vibration frequencies at the transition state, and $\nu_{ \pm}$is the imaginary vibration frequency at the transition state. All vibration frequencies are given in $T H z$. Values in Zhang et al. [17] are listed in parentheses for comparison.

\begin{tabular}{ccccccc}
\hline Diffusion Step & $\nu_{1}$ & $\nu_{2}$ & $\nu_{3}$ & $\nu_{1}^{T S}$ & $\nu_{2}^{T S}$ & $v_{ \pm}$ \\
\hline \hline \multirow{2}{*}{$\mathrm{T}-\mathrm{T}$} & 37.08 & 37.08 & 33.62 & 43.79 & 43.74 & 18.46 \\
& $(36.87)$ & $(36.87)$ & $(33.06)$ & $(43.06)$ & $(43.04)$ & $(17.92)$ \\
\multirow{2}{*}{$\mathrm{T}-\mathrm{O}$} & 37.08 & 37.08 & 33.62 & 44.30 & 41.60 & 16.45 \\
& $(36.87)$ & $(36.87)$ & $(33.06)$ & $(45.80)$ & $(42.66)$ & $(17.59)$ \\
$\mathrm{O}-\mathrm{T}$ & 23.50 & 21.04 & 21.04 & 44.30 & 41.60 & 16.45 \\
& $(23.32)$ & $(20.84)$ & $(20.84)$ & $(45.80)$ & $(42.66)$ & $(17.59)$ \\
$\mathrm{O}-\mathrm{O}$ & 23.50 & 21.04 & 21.04 & 47.39 & $47.37)$ & 9.76 \\
& $(23.32)$ & $(20.84)$ & $(20.84)$ & $(47.30)$ & $(47.29)$ & $(9.95)$ \\
\hline
\end{tabular}




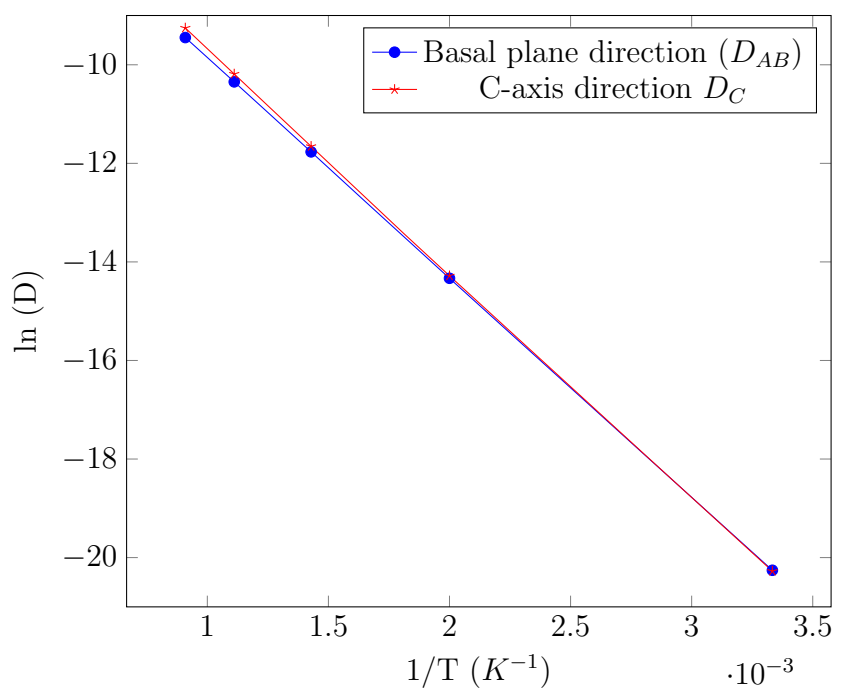

Figure 4.2: Bulk diffusivity vs temperature computed from KMC simulations for $\mathrm{H}$ diffusion in pure $\mathrm{Zr}$

Table 4.3: Diffusivity parameters for $\mathrm{H}$ diffusion in $\mathrm{Zr}$

\begin{tabular}{ccc}
\hline Direction & $D_{0}\left(\mathrm{~cm}^{2} / w\right)$ & $E_{a}(\mathrm{eV})$ \\
\hline \hline Basal plane $\left(D_{A B}\right)$ & $4.535 \times 10^{-3}$ & 0.384 \\
C-axis direction $\left(D_{C}\right)$ & $5.811 \times 10^{-3}$ & 0.392 \\
\hline
\end{tabular}

ranging between $300 K-1100 K$ in steps of $200 K$, with 100,000 KMC runs for each temperature. The time for each KMC run was selected such that each KMC run lasted more than 1,000,000 diffusion steps in pure Zr. For 100,000 KMC runs, the average diffusivity estimate converged to less than $0.1 \%$.

Plotting the logarithm of diffusivity against the inverse of temperature (see Fig. 4.2) we obtained the pre-exponent of diffusivity $D_{0}$ and the activation energy $E_{a}$ for the basal plane and the c-axis direction as shown in Table 4.3. These values agree somewhat well with the experimental values available for $\mathrm{Zr}$, where the differences could be attributed to the various defects and impurities in the material as shown in Zhang et al. [17]. In this study we only looked at the effect of interstitial $\mathrm{O}$ on diffusivity, hence we neglected the influence of other impurities and defects.

From these results we see that $\mathrm{H}$ diffusion in $\mathrm{Zr}$ is closely isotropic, especially near ambient temperature. The anisotropy of $\mathrm{H}$ diffusion $\left(D_{C} / D_{A B}\right.$, where $D_{C}$ and 
$D_{A B}$ are the diffusivities in $\mathrm{C}$-axis and basal plane directions respectively) varies between $0.93-1.18$ in the considered temperature range. Reasons for this variation are discussed in one of our previous papers [24].

\subsubsection{Hydrogen diffusion in $\mathrm{Zr}$ with interstitial oxygen Effect of oxygen on neighboring interstitial sites}

For the DFT calculations, we used two $4 \times 4 \times 4 \mathrm{Zr}$ supercells with one and eight $\mathrm{O}$ atoms respectively. In the first system $\mathrm{H}$ occupying each interstitial site is only affected by one $\mathrm{O}$ atom. For ease of referencing we indexed each distinct interstitial site using the number of steps $i$ to the $\mathrm{O}$ in the basal plane and the number of steps $j$ to the plane containing the nearest $\mathrm{O}$ along the $\mathrm{C}$-axis. $\mathrm{T}_{i, j}$ and $\mathrm{O}_{i, j}$ indicate tetrahedral and octahedral interstitial sites respectively. As shown in Fig. 4.3 and Fig. 4.4, the $i$ and $j$ indices increase as the distance from the $\mathrm{O}$ atom increases in basal plane and $\mathrm{C}$-axis direction respectively. Octahedral sites are distributed symmetrically along both sides of $\mathrm{O}$ in the $\mathrm{C}$-axis direction, while for tetrahedral sites, the variation is not symmetric. So we considered the $j$ indices in direction of nearest neighbour site to be positive and the other direction to be negative for the sake of noting the different steps in the following results.

We calculated the relative change in energy of these interstitial sites when compared to a $\mathrm{H}$ occupying the respective interstitial sites in $\mathrm{Zr}$ as

$$
\Delta E=E_{Z r_{O, H}}-E_{Z r_{O}}-\left(E_{Z r_{H}}-E_{Z r}\right)
$$

where $E_{Z r_{O, H}}, E_{Z r_{O}}, E_{Z r_{H}}$, and $E_{Z r}$ are energies of supercell containing both $\mathrm{O}$ and $\mathrm{H}$, just $\mathrm{O}, \mathrm{H}$ in the relevant interstitial site (tetrahedral or octahedral), and no impurities respectively. Fig. 4.5 shows the variation of $\Delta E$ when only a single $\mathrm{O}$ is present. The distances from the $\mathrm{O}$ atom to the tetrahedral sites are taken as positive for $j>0$ and negative for $j<0$. The variation shows a similarity to a classical attractive well, with $\Delta E$ high for very short distances, minimum at some distance, and going to zero at large distances. It shows that the nearest neighboring site to the $\mathrm{O}$ atom $\mathrm{T}_{1,1}$ has a considerably higher energy making it less stable. $\mathrm{O}$ is known to block the nearest neighbor tetrahedral sites for $\mathrm{H}$ occupancy [3] which is consistent with the current 


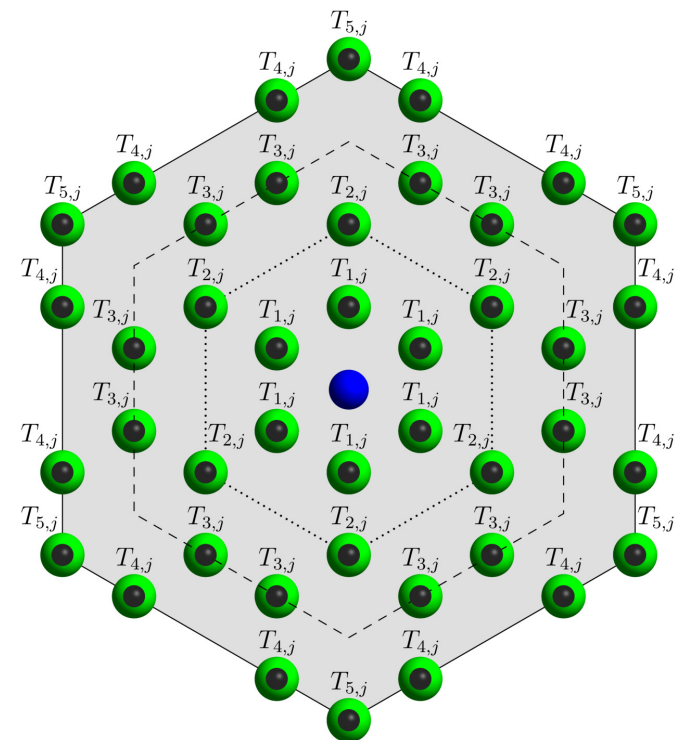

(a) Naming convention for tetrahedral sites within a basal plane $j$

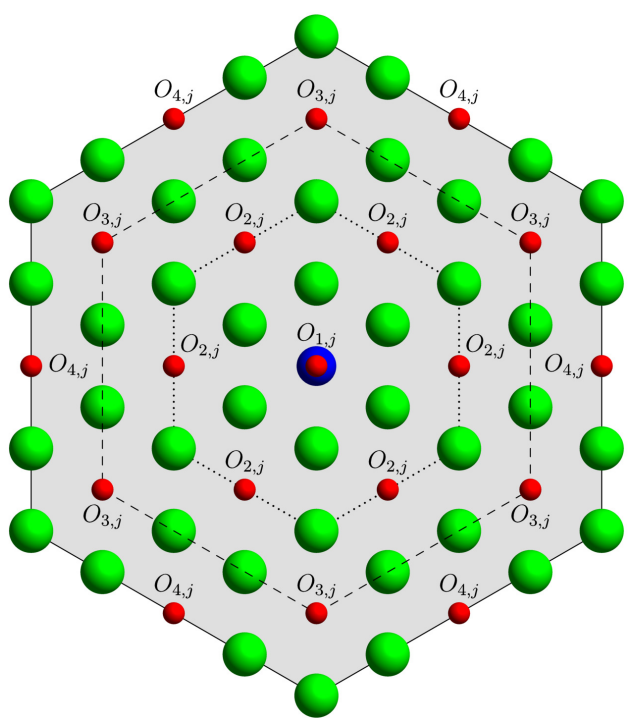

(b) Naming convention for octahedral sites within a basal plane $j$

Figure 4.3: Variation of index $i$ for tetrahedral and octahedral sites for different O concentrations. Zr, O, tetrahedral sites, and octahedral sites are shown by green, blue, black and red spheres respectively. The region attributed for a single $\mathrm{O}$ atom in $4 \times 4 \times 4,3 \times 3 \times 3$, and $2 \times 2 \times 2 \mathrm{Zr}$ supercells are bounded by solid lines, dashed lines, and dotted lines respectively 


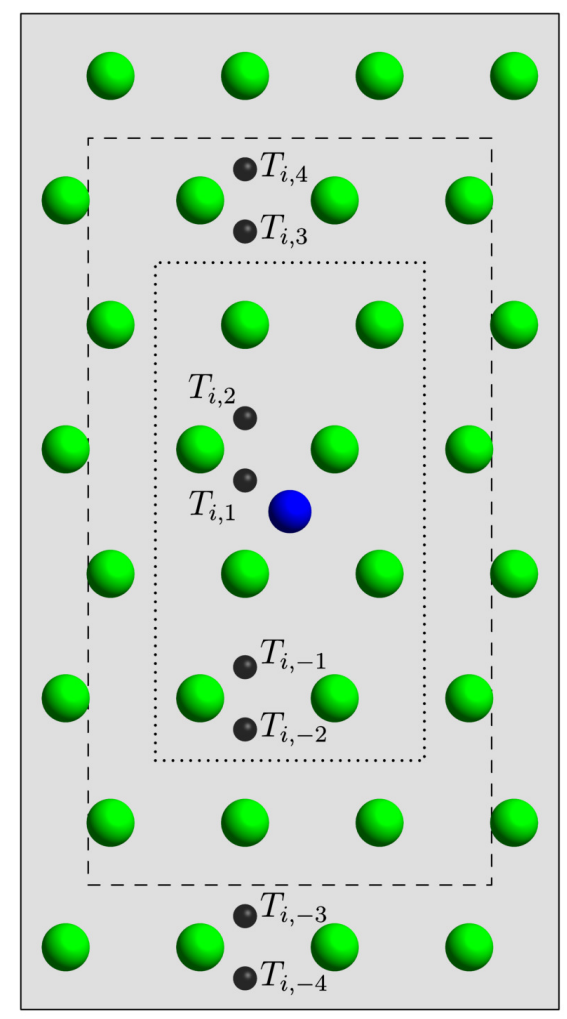

(a) Naming convention for tetrahedral sites for any $i$

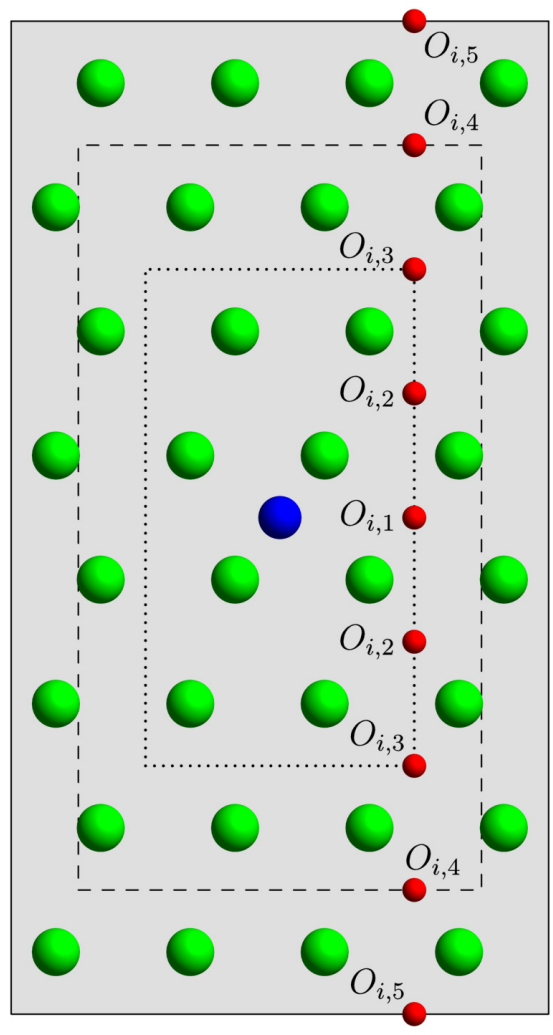

(b) Naming convention for octahedral sites for any $i$

Figure 4.4: Variation of index $j$ for tetrahedral and octahedral sites for different O concentrations for any $i$. Colours and bound regions are the same as the previous figure 


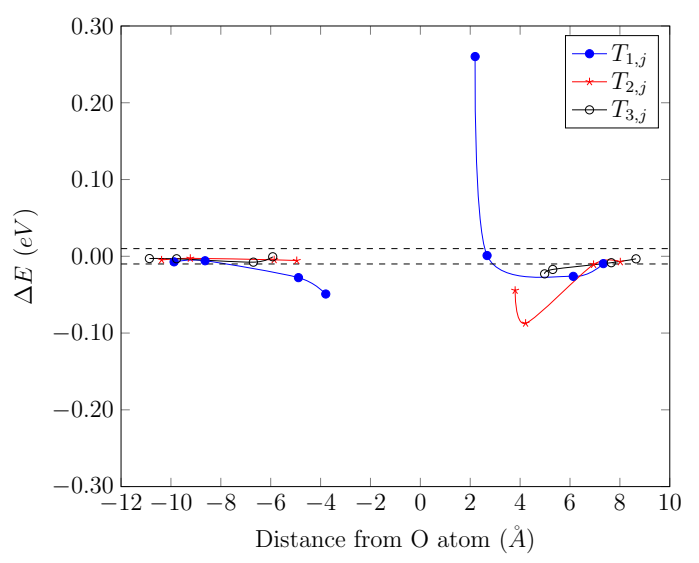

(a) Tetrahedral sites

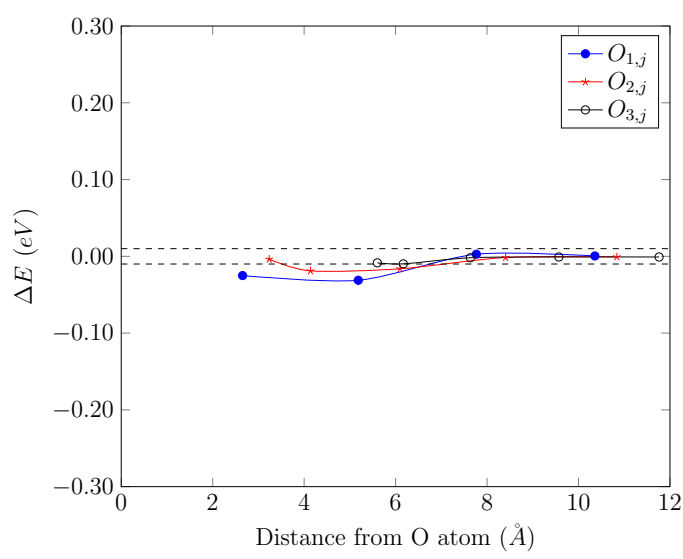

(b) Octahedral sites

Figure 4.5: Variation of $\Delta E$ for interstitial sites when only one $\mathrm{O}$ atom is present in the supercell

results. We also noticed a sharp decrease in energy in the $\mathrm{T}_{2,2}$ site making it the most stable location. To simplify the NEB calculations to be preformed, when $\Delta E$ converges to below $0.01 \mathrm{eV}$ we considered the effect of the $\mathrm{O}$ atom to be negligible and the behaviour of $\mathrm{H}$ at these sites to be similar to that of $\mathrm{H}$ in Pure Zr. NEB and the central difference method were used to obtain the activation energies and the vibration frequencies for all the distinct hops. These results were used to find the thermally activated hopping rates from SC-HTST to input in the KMC simulations. One important thing noticed during this step is that the ZPE corrected activation energy for the transition from $T_{1,1}$ to $T_{1,2}$ becomes negative, which means that $T_{1,1}$ is not a stable occupancy location and will automatically relax to the $T_{1,2}$ site, making $\mathrm{T}_{1,1}$ a part of the $\mathrm{T}_{1,2}$ energy basin.

The region affected by an $\mathrm{O}$ atom is as shown in Fig. 4.6, where the polyhedron encompasses all the sites for which $\Delta E>0.1 \mathrm{eV}$. This can be simplified to a cylindrical region for ease of representation. If we look at the distribution of affected regions when an $\mathrm{O}$ atom is present in each of $4 \times 4 \times 4$ and $3 \times 3 \times 3 \mathrm{Zr}$ unit cells, the arrangement of neighbouring regions are shown in Fig. 4.7. For the $4 \times 4 \times 4$ system, the affected regions are spread apart where each interstitial site is at most affected by a single $\mathrm{O}$ atom, allowing us to use the calculated hopping rates to simulate $\mathrm{H}$ diffusivity for this $\mathrm{O}$ concentration. In the $3 \times 3 \times 3$ system we can see that the that the regions are touching each other in the basal plane direction, but this will only 


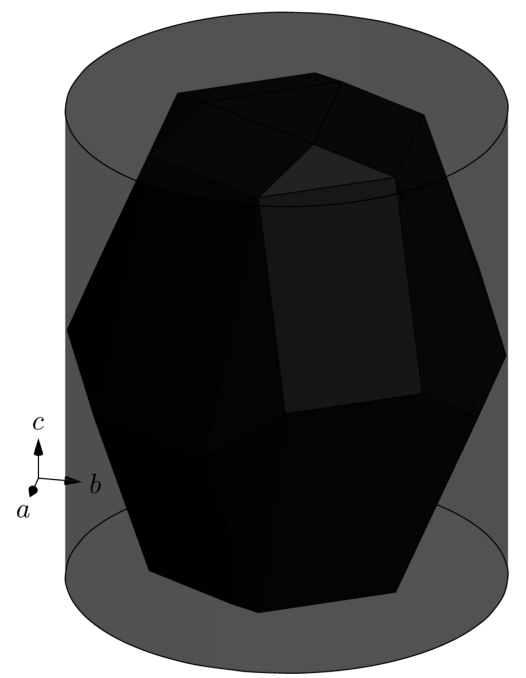

Figure 4.6: Region affected by a single $O$ atom which can be simplified to the cylindrical region for ease of representation

affect $T_{3,1}$ interstitial sites in the perimeter of the region, and hence we still can use the hopping rates calculated without a significant effect.

For the second system we considered eight $\mathrm{O}$ atoms in a $4 \times 4 \times 4 \mathrm{Zr}$ supercell (equivalent to one $\mathrm{O}$ atom in $2 \times 2 \times 2$ supercell), where from previous results we can see that some interstitial sites will be affected by multiple $\mathrm{O}$ atoms. Similar to the above system we looked at the variation of $\Delta E$ for the distinct interstitial locations. As Fig. 4.8 shows, we can see that all the interstitial sites are affected by the presence of $\mathrm{O}$ atoms. Similar to the single $\mathrm{O}$ system, we can see that $\mathrm{T}_{1,1}$ sites are quite unstable. $\mathrm{T}_{2,2}$ sites are still the most stable sites, with the energetic favorability having increased further. Activation energies and vibration frequencies obtained from NEB and central difference method for each distinct transition for both systems are provided in the supplemental material, and were used to determine the thermally activated hopping rates for each transition.

\section{Effect of oxygen on diffusivity}

Using the hopping rates determined, we performed KMC simulations for the three difference $\mathrm{O}$ concentrations. Variations of diffusivity for each concentrations are as shown in Fig. 4.9 for the basal plane and c-axis directions. As can be seen from this 


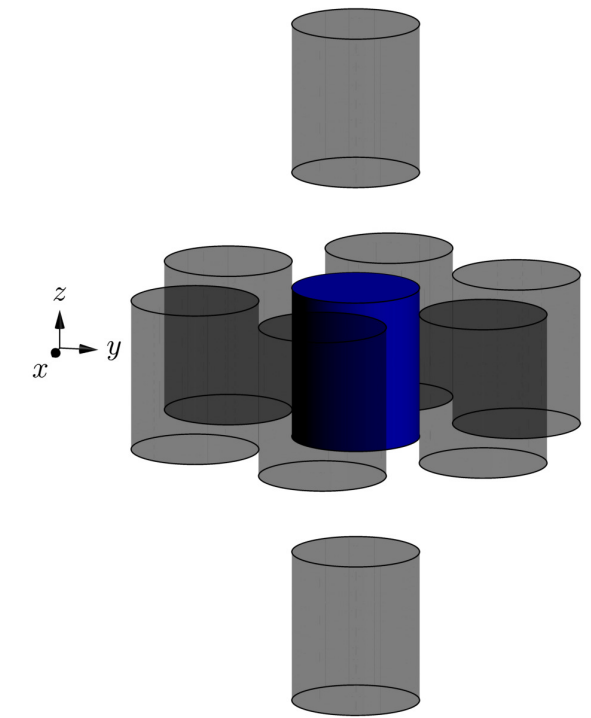

(a) With one $\mathrm{O}$ per $4 \times 4 \times 4$ supercell

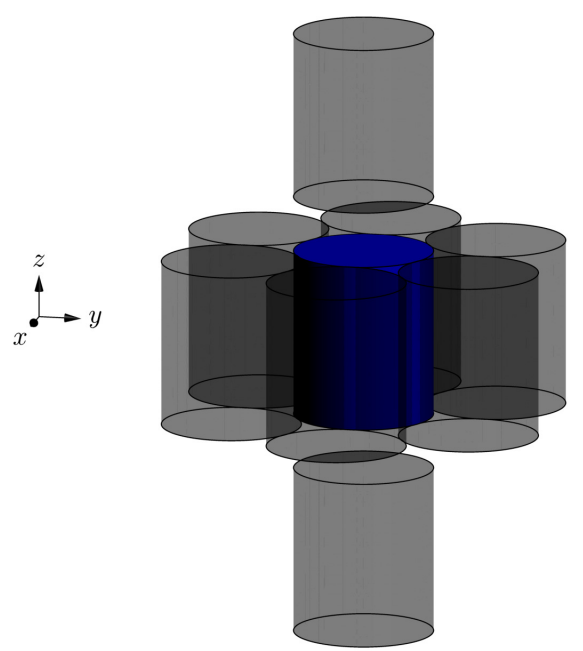

(b) With one $\mathrm{O}$ per $3 \times 3 \times 3$ supercell

Figure 4.7: Arrangement of neighbouring regions by $\mathrm{O}$ atoms

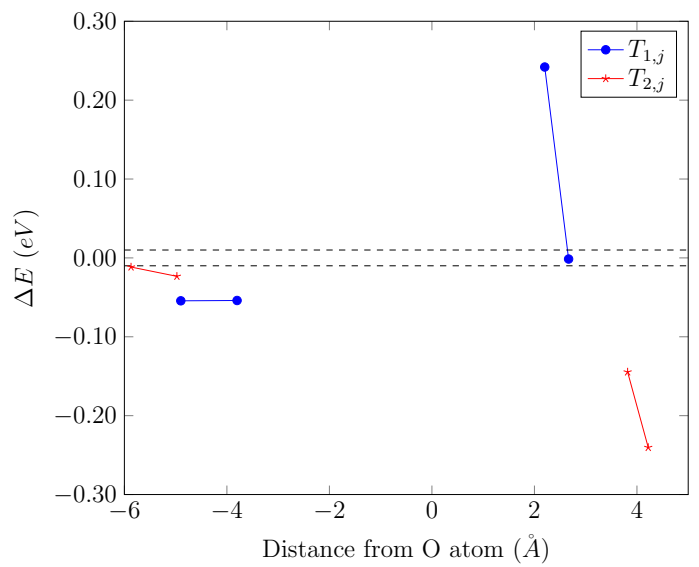

(a) Tetrahedral sites

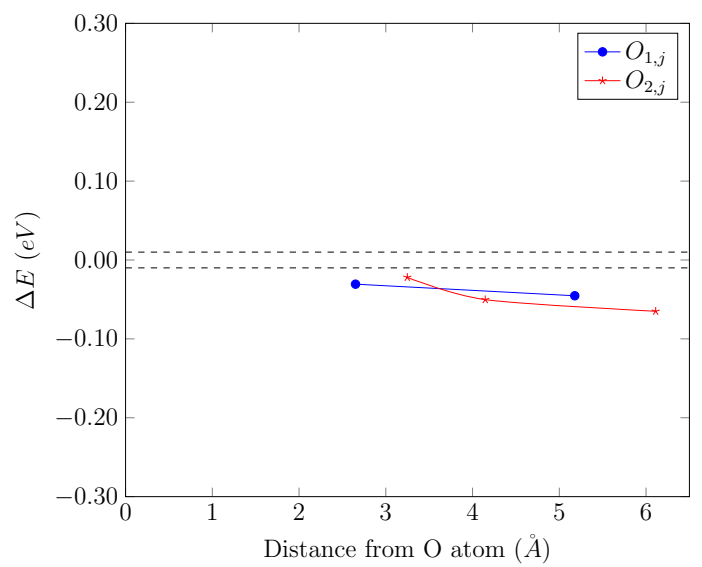

(b) Octahedral sites

Figure 4.8: Variation of $\Delta E$ for interstitial sites when eight $\mathrm{O}$ atoms are present in the supercell 


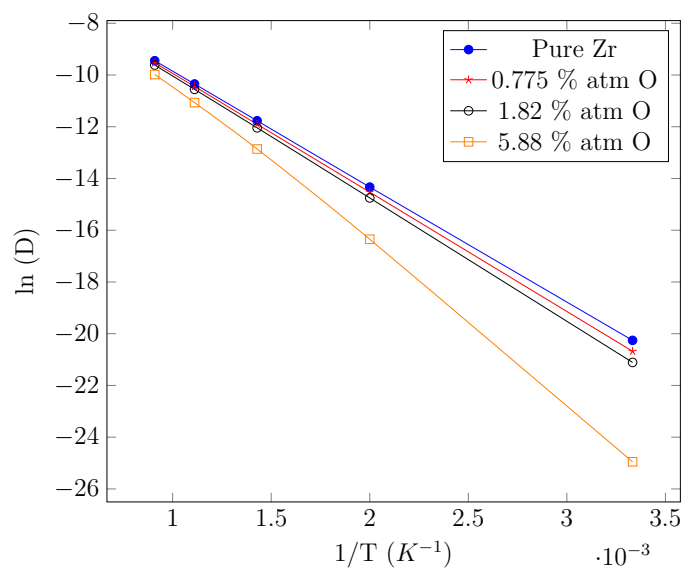

(a) Basal plane direction

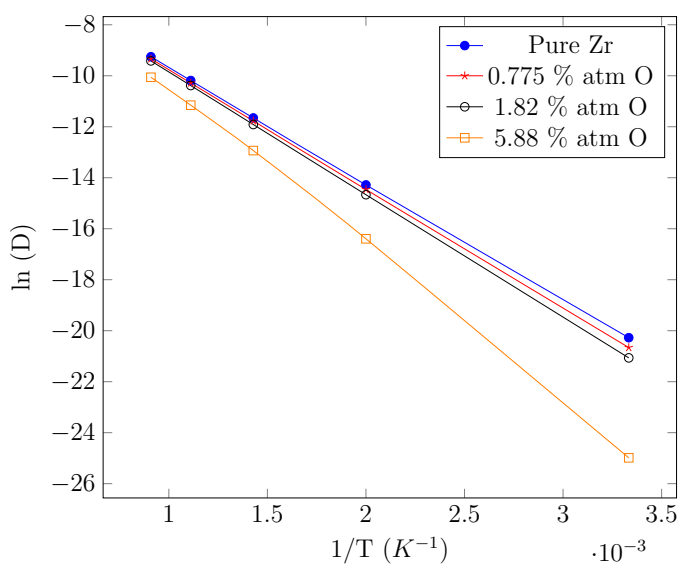

(b) C-axis direction

Figure 4.9: Variation of diffusivity with $\mathrm{O}$ concentration

graph when the $\mathrm{O}$ concentration increases the $\mathrm{H}$ diffusivity reduces, supporting the hypothesis of the current study. We can see that at the highest $\mathrm{O}$ concentration the relationships are not linear, deviating from the Arrhenius form

$$
D=D_{0} \exp \left(\frac{-E_{a}}{k_{B} T}\right)
$$

while for low concentrations they become close to the Arrhenius form.

We calculated the diffusivity parameters $D_{0}$ and $E_{a}$ for $\mathrm{H}$ diffusion as shown in Table 4.4 and Table 4.5 for the basal plane and C-axis directions respectively. If we look at the change in diffusivity with respect to the diffusivity in pure $\mathrm{Zr}$ (see Fig. 4.10), we can see that the decrease in diffusivity is influenced by both the temperature and the $\mathrm{O}$ concentration. This is evident by the higher activation energy through increased $\mathrm{O}$ concentration.

To identify the reasons for this variation we looked at the diffusion paths taken by $\mathrm{H}$ atoms for each of the $\mathrm{O}$ concentrations at $300 \mathrm{~K}$, where the highest difference in the diffusivity is observed. At the lowest $\mathrm{O}$ concentration $(0.775 \% \mathrm{~atm})$, about $60 \%$ of the diffusion steps occur between interstitial sites unaffected by the $\mathrm{O}$ atom. This can be expected since the majority of the interstitial sites at this concentration are out of the influence from $\mathrm{O}$ atoms (more than $70 \%$ of both tetrahedral and octahedral 
Table 4.4: Diffusivity parameters for $\mathrm{H}$ diffusion in the basal plane direction for different $\mathrm{O}$ concentrations

\begin{tabular}{ccc}
\hline O concentration & $D_{0}\left(\mathrm{~cm}^{2} / w\right)$ & $E_{a}(\mathrm{eV})$ \\
\hline \hline Pure Zr & $4.535 \times 10^{-3}$ & 0.384 \\
$0.775 \%$ atm O & $4.828 \times 10^{-3}$ & 0.397 \\
$1.82 \%$ atm O & $5.089 \times 10^{-3}$ & 0.409 \\
$5.88 \%$ atm O & $15.945 \times 10^{-3}$ & 0.535 \\
\hline
\end{tabular}

Table 4.5: Diffusivity parameters for $\mathrm{H}$ diffusion in the $\mathrm{C}$-axis direction for different O concentrations

\begin{tabular}{ccc}
\hline O concentration & $D_{0}\left(\mathrm{~cm}^{2} / \mathrm{w}\right)$ & $E_{a}(\mathrm{eV})$ \\
\hline \hline Pure Zr & $5.811 \times 10^{-3}$ & 0.392 \\
$0.775 \%$ atm O & $6.109 \times 10^{-3}$ & 0.403 \\
$1.82 \%$ atm O & $6.427 \times 10^{-3}$ & 0.414 \\
$5.88 \%$ atm O & $14.402 \times 10^{-3}$ & 0.533 \\
\hline
\end{tabular}

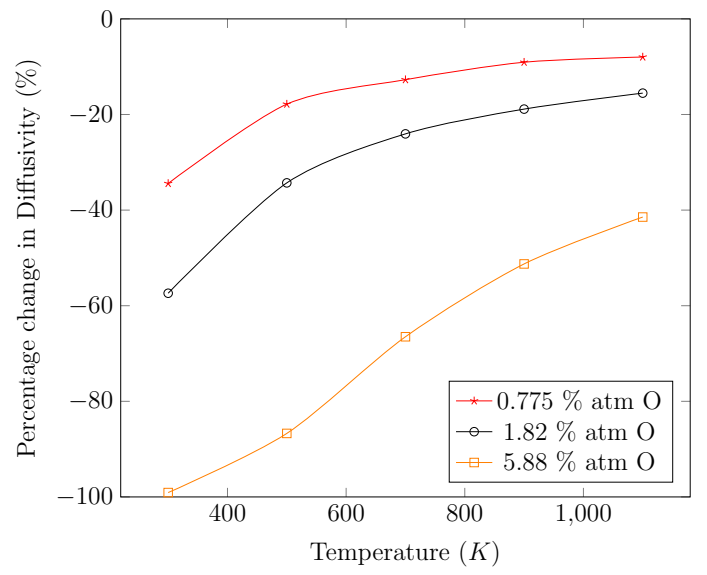

(a) Basal plane direction

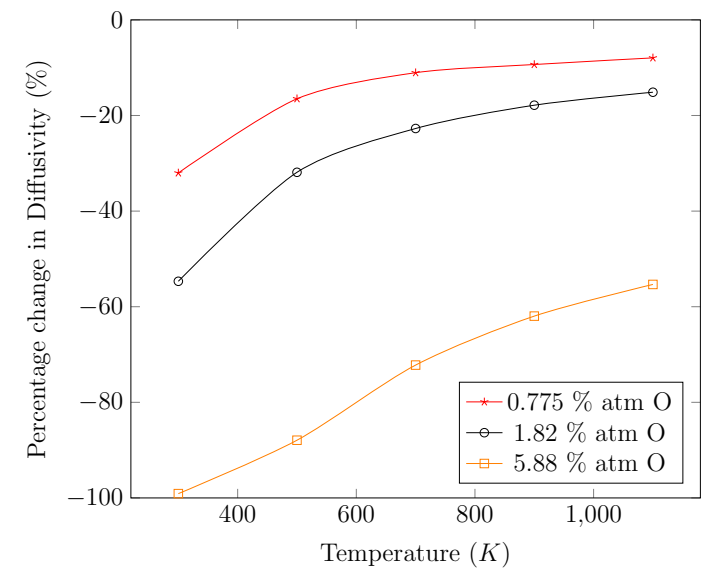

(b) C-axis direction

Figure 4.10: Percentage change in diffusivity compared to that of Pure Zr 


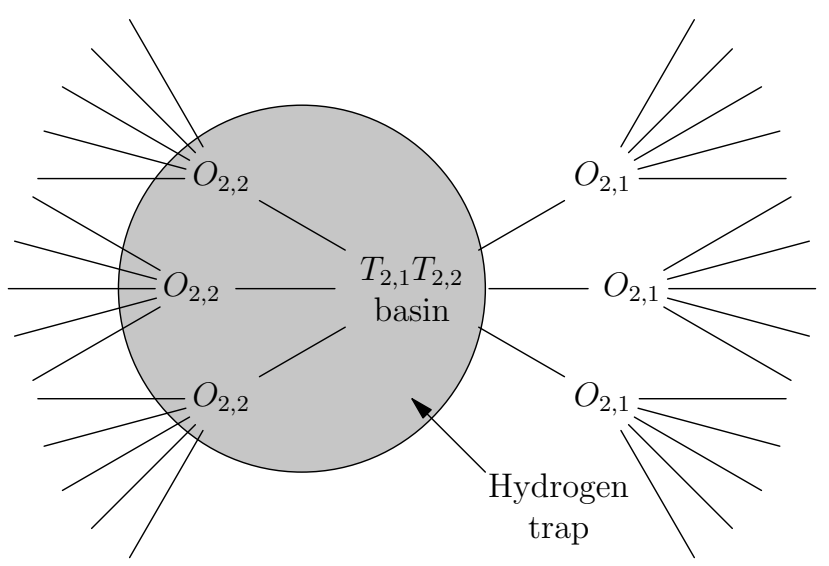

Figure 4.11: Trap formed by pseudo basin $\mathrm{T}_{2,1} T_{2,2}$ and $\mathrm{O}_{2,2}$ sites. $\mathrm{H}$ atoms arriving in these traps will flicker between the sites before escaping, causing a reduction in diffusivity

sites respectively). At $1.82 \%$ atm O concentration, only $20 \%$ of the transitions are among these sites, even though still about $45 \%$ of tetrahedral sites and $35 \%$ of the octahedral sites are not under the influence of $\mathrm{O}$ atoms. This shows that $\mathrm{H}$ prefers the influenced region for occupancy, making these regions trapping sites for $\mathrm{H}$ atoms.

For the given time interval, the number of diffusion jumps reduces from that of pure Zr by approximately $26 \%, 42 \%$, and $94 \%$ as the $\mathrm{O}$ concentration increases. This compares well to the reduction in diffusivities of approximately $35 \%, 57 \%$, and $99 \%$ respectively. This is as expected since it takes more energy to remove an atom from a more stable location. The additional reduction is due to the flickering (back and forth movement between two or more neighboring sites) events happening between the sites in the affected region which is very much noticeable in the system with the highest $\mathrm{O}$ concentration.

In this system, transitions are mainly among $\mathrm{O}_{2,2}$ sites and pseudo basins formed by adjacent $\mathrm{T}_{2,1}$ and $\mathrm{T}_{2,2}$ sites, accounting for more than $80 \%$ of the transitions. Each set of this pseudo basin and the three nearest neighbour $\mathrm{O}_{2,2}$ sites forms a sink for $\mathrm{H}$ atoms where they are trapped between these sites for several diffusion steps before escaping it (see Fig. 4.11). This effect is similar to the pseudo basins formed by nearest neighbour tetrahedral sites, but to a smaller extent. This causes the diffusivity at this concentration to reduce further. 


\subsubsection{Implications of the Diffusivity on Hydride Precipita- tion}

We have quantified the effect $\mathrm{O}$ concentration has on the diffusivity of $\mathrm{H}$ in $\alpha$-Zr using a combination of first principles calculations and KMC simulations. Motivation for this study was to determine how this would affect the sizes of precipitates formed in $\mathrm{Zr}$ vessels and to check the validity of the assumption that $\mathrm{O}$ slows down the $\mathrm{H}$ movement considerably to form very small hydrides (making them difficult to be noticed through optical methods). If the size if the precipitates (hydrides) depend on the catchment volume, the size of the precipitates will be proportional to $D^{1.5}$. If we look at how this value changes with the $\mathrm{O}$ concentration we can understand the size variation to be expected in the precipitate sizes. Fig. 4.12 shows the variation of $D^{1.5}$ for different $\mathrm{O}$ concentrations we considered for the temperature range $100^{\circ} \mathrm{C}-300^{\circ} \mathrm{C}$ (which is the operating condition of most reactors). Fig. 4.13 looks at the relative size of precipitates with respect to the size of precipitates expected in pure $\mathrm{Zr}$, which would be the ratio $\left(D / D_{Z r}\right)^{1.5}$ where $D$ is the diffusivity of $\mathrm{H}$ for the respective $\mathrm{O}$ concentration and $D_{Z r}$ is the diffusivity of $\mathrm{H}$ in pure $\mathrm{Zr}$ at the same temperature. From this we can see a clear decrease for precipitates when $\mathrm{O}$ concentration is $5.88 \%$ atm. For the other two concentrations we still can see a significant difference but not as significant as when $\mathrm{O}$ concentration is $5.88 \%$ atm. To have a noticeable decrease in the size of the precipitates they have to be smaller by a magnitude in the order of $10^{-2}$ (0.01 times). We can only see this in the case with $5.88 \%$ atm $\mathrm{O}$ concentration.

\section{Oxygen Concentration Profile}

We looked at the $\mathrm{O}$ concentration profiles in these $\mathrm{Zr}$ vessels through a model proposed by Denis et al. [25]. This model calculates the diffusion of $\mathrm{O}$ from a surface oxygen layer at a known depth within a certain time using diffusion rates for $\mathrm{O}$ in the Oxide and Zr. As the starting oxide layer, a thickness of $5 \mu \mathrm{m}$ was used since it's been found that the oxide layer thickness in the outer surface varies between $2-5 \mu \mathrm{m}$ [26]. Diffusivity values for $\mathrm{O}$ in pure $\mathrm{Zr}$ and the oxide was used as $2.72 \times 10^{-19} \mathrm{~m}^{2} / \mathrm{s}$ [27] and $4.27 \times 10^{-19} \mathrm{~m}^{2} / \mathrm{s}$ [28] respectively. We determined the $\mathrm{O}$ concentration profile as shown in Fig. 4.14 when the temperature is $300{ }^{\circ} \mathrm{C}$ after a period of 30 years. The initial oxygen concentration at the the Oxide interface is approximately $27.4 \%$ 


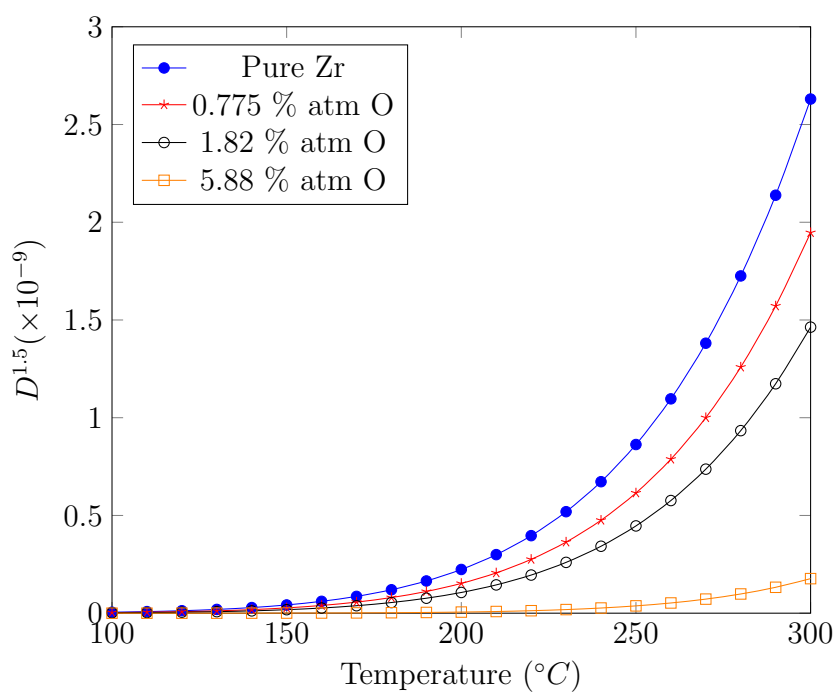

Figure 4.12: Variation of $D^{1.5}$ with the temperature for temperatures between $100^{\circ} \mathrm{C}$ and $300^{\circ} \mathrm{C}$

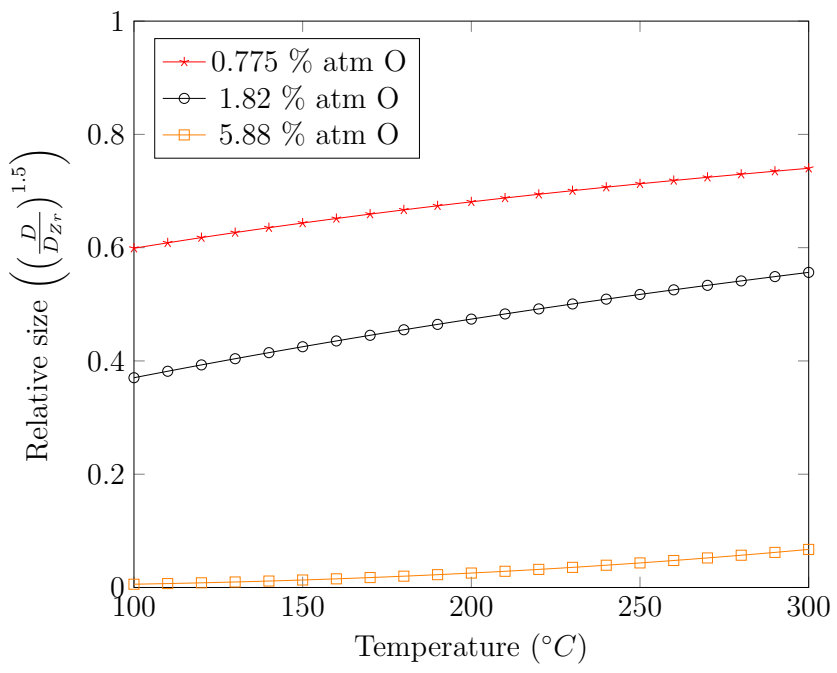

Figure 4.13: Relative size of the precipitates to the size of precipitates expected in pure $\mathrm{Zr}$ for temperatures between $100^{\circ} \mathrm{C}$ and $300^{\circ} \mathrm{C}$. $\mathrm{D}$ is the diffusivity of $\mathrm{H}$ for the respective $\mathrm{O}$ concentration and $D_{Z r}$ is the diffusivity of $\mathrm{H}$ in pure $\mathrm{Zr}$ at the same temperature 


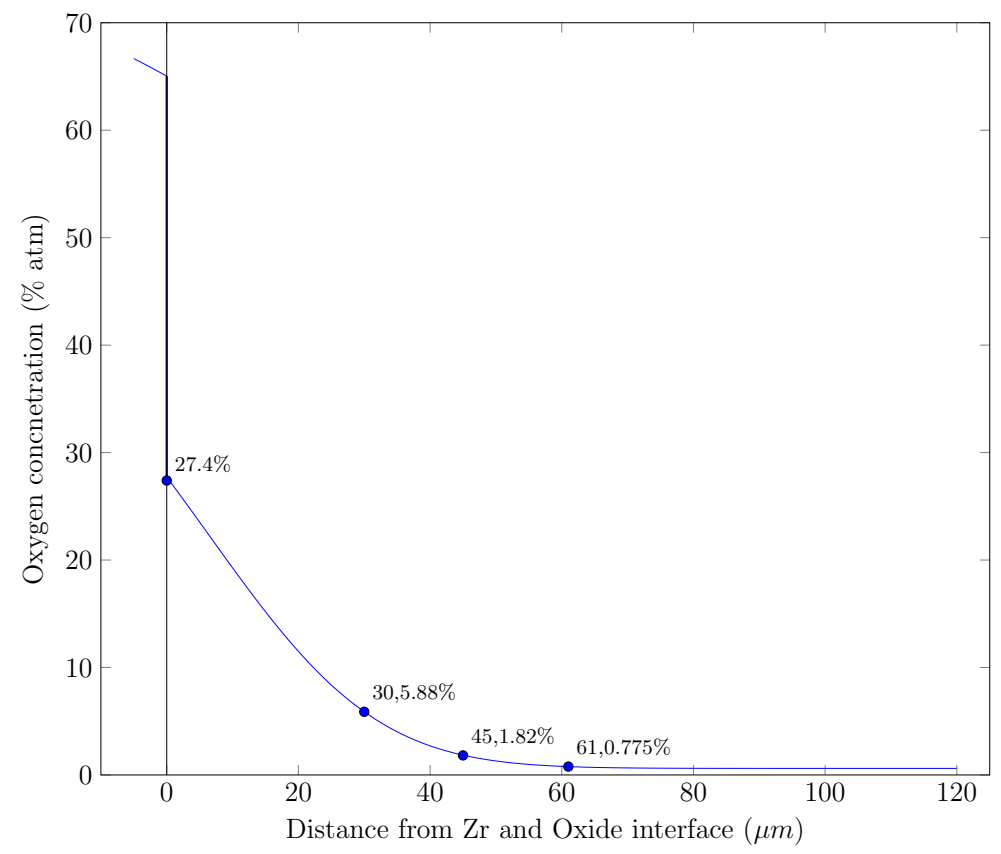

Figure 4.14: Oxygen concentration profile in Zirconium after 30 year at $290^{\circ} \mathrm{C}$

atm [29], which is the terminal solubility level for $\mathrm{O}$ in $\alpha$-Zr. As the distance from the interface increases, $\mathrm{O}$ concentration drops and reaches the average bulk concentration value of $0.6 \%$ atm (see Fig. 4.14).

Looking at this $\mathrm{O}$ concentration profile, we see that $\mathrm{O}$ concentration reaches 5.88 $\%$ atm. value at a approximately $30 \mu \mathrm{m}$ from the interface. O concentration reaches $1.82 \%$ atm and $0.775 \%$ atm (the other $\mathrm{O}$ concentrations we considered in the study) at approximately $45 \mu \mathrm{m}$ and $61 \mu \mathrm{m}$ respectively. Comparing these distances with the denuded zone thickness (which can reach about $100-200 \mu \mathrm{m}$ ) the reach of oxygen concentration is quite low. With this we can see that the amount of oxygen present in the denuded zones is not sufficient to slow the diffusion rates of $\mathrm{H}$ to cause significant reduction in the sizes of hydrides formed. Hence, we can conclude that the (primary) reason for the formation of denuded zones is unlikely to be the reduction in the diffusion rates by the interstitial $\mathrm{O}$.

\subsection{Conclusions and Future Direction}

We looked at diffusivity of $\mathrm{H}$ in $\mathrm{Zr}$ with and without the influence of $\mathrm{O}$ using a combination of first principles methods and KMC simulation. The first principles 
calculations have been successfully used to determine the hopping rates for individual diffusion steps, validated from previous work carried out for $\mathrm{H}$ diffusion in pure $\mathrm{Zr}$. This study shows that $\mathrm{H}$ diffusion in $\mathrm{Zr}$ can be decreased by the presence of interstitial $\mathrm{O}$ and further decrease as the $\mathrm{O}$ concentration increases. We looked at the paths taken by $\mathrm{H}$ atoms and found that in addition to the decrease in hopping rates, $\mathrm{H}$ traps are formed by the amalgamation of occupancy sites, decreasing the diffusivity.

While this study proves that diffusivity of $\mathrm{H}$ in $\mathrm{Zr}$ is decreased by $\mathrm{O}$, the amount of reduction in the diffusivity is not seen to be sufficient to decrease the size of the precipitates to be noticeable in the denuded zones. The $\mathrm{O}$ concentration profile suggests that the amount of $\mathrm{O}$ present in the denuded zone is not sufficient to decrease the diffusivity to a sufficient level to decrease the size of precipitates noticeably. Hence, our results oppose the hypothesis that denuded zones are formed due to the decrease of hydrogen diffusivity caused by interstitial oxygen.

This gives rise to the need to look at other mechanisms which could explain the formation of denuded zones in Zr pressure vessels. In Chapter 5 we are presenting how the stress gradients found in these pressure tubes will affect the diffusion to see whether it has an effect on the formation of denuded zones.

\section{Bibliography}

[1] H. Muta, Y. Etoh, Y. Ohishi, K. Kurosaki, and S. Yamanaka, "Ab initio study of hydrogen diffusion in zirconium oxide," Journal of Nuclear Science and Technology, vol. 49, no. 5, pp. 544-550, 2012.

[2] J. T. Fermann and S. Auerbach, "Modeling proton mobility in acidic zeolite clusters: II. room temperature tunneling effects from semiclassical rate theory," J. Chem. Phys., vol. 112, pp. 6787-6794, 2000.

[3] D. Hardie, "The effect of oxygen on the precipitation of hydrogen from zirconium," Journal of Nuclear Materials, vol. 17, pp. 88-90, 1965.

[4] R. Krishnan and M. K. Asundi, "Zirconium alloys in nuclear technology," in Proceedings of the Indian Academy of Sciences Section C: Engineering Sciences, vol. 4, pp. 41-56, 1981.

[5] S. Banerjee, "Nuclear applications: Zirconium alloys," in Encyclopedia of Materials: Science and Technology (K. Buschow, R. Cahn, M. Flemings, B. Ilschner, E. Kramer, S. Mahajan, and P. Veyssiere, eds.), pp. 6287-6299, Elsevier, 2 ed., 2001. 
[6] D. Féron, "Overview of nuclear materials and nuclear corrosion science and engineering," in Nuclear Corrosion Science and Engineering (D. Féron, ed.), ch. 2, pp. 31-56, Woodhead Publishing, 2012.

[7] G. McRae, C. Coleman, and B. Leitch, "The first step for delayed hydride cracking in zirconium alloys," J. Nucl. Mater., vol. 396, pp. 130-143, 2010.

[8] M. De Las Heras, S. Parodi, L. Ponzoni, J. Mieza, S. Müller, S. Alcantar, and G. Domizzi, "Effect of thermal cycles on delayed hydride cracking in Zr-2.5Nb alloy," J. Nucl. Mater., vol. 509, pp. 600-612, 2018.

[9] T. N. Murty, R. Singh, and P. Ståhle, "Delayed hydride cracking of Zr-2.5\%nb pressure tube material due to partially constrained precipitates," J. Nucl. Mater., vol. 513, pp. 129-142, 2019.

[10] C. Roy, "An experiment to clarify the effect of dissolved oxygen on the terminal solubility of hydrogen in zirconium," Journal of Nuclear Materials, vol. 13, pp. 275-277, 1964.

[11] G. Kresse and J. Hafner, "Ab initio molecular dynamics for liquid metals," Physical Review B, vol. 47, no. 1, pp. 558-561, 1993.

[12] G. Kresse and J. Furthmüller, "Efficiency of ab-initio total energy calculations for metals and semiconductors using a plane-wave basis set," Computational Materials Science, vol. 6, no. 1, pp. 15-50, 1996.

[13] G. Kresse and J. Furthmüller, "Efficient iterative schemes for $<\mathrm{i}>$ ab initio $</ \mathrm{i}>$ total-energy calculations using a plane-wave basis set," Physical Review B, vol. 54, no. 16, pp. 11169-11186, 1996.

[14] J. P. Perdew, K. Burke, and M. Ernzerhof, "Generalized Gradient Approximation Made Simple," Physical Review Letters, vol. 77, no. 18, pp. 3865-3868, 1996.

[15] G. Henkelman and H. Jónsson, "Improved tangent estimate in the nudged elastic band method for finding minimum energy paths and saddle points Improved tangent estimate in the nudged elastic band method for finding minimum energy paths and saddle points," Journal of Chemical Physics, vol. 113, pp. 9978-9985, 2000 .

[16] B. Bhatia and D. S. Sholl, "Quantitative assessment of hydrogen diffusion by activated hopping and quantum tunneling in ordered intermetallics," Phys. Rev. $B$, vol. 72, p. 224302, 2005.

[17] Y. Zhang, C. Jiang, and X. Bai, "Anisotropic hydrogen diffusion in $\alpha$-Zr and Zircaloy predicted by accelerated kinetic Monte Carlo simulations," Scientific Reports, vol. 7, pp. 41033 1-13, 2017.

[18] B. Puchala, M. L. Falk, and K. Garikipati, "An energy basin finding algorithm for kinetic monte carlo acceleration," J. Chem. Phys., vol. 132, p. 134104, 2010.

[19] J. P. Pemsler, "Diffusion of oxygen in zirconium and its relation to oxidation and corrosion," Journal of the Electrochemical Society, vol. 105, pp. 315-322, 1958. 
[20] M. V. Glazoff, "Modeling of some physical properties of zirconium alloys for nuclear applications in support of ufd campaign," tech. rep., Idaho National Laboratory, UFD Campaign, Idaho Falls, Idaho, Aug 2013.

[21] C. Domain, R. Besson, and A. Legris, "Atomic-scale ab-initio study of the zrh system: I. bulk properties," Acta Materialia, vol. 50, no. 13, pp. 3513-3526, 2002.

[22] P. A. Burr, S. T. Murphy, S. C. Lumley, M. R. Wenman, and R. W. Grimes, "Hydrogen accommodation in zr second phase particles: implications for h pickup and hydriding of zircaloy-2 and zircaloy-4," Corrosion Science, vol. 69, pp. 14, 2013.

[23] M. Christensen, W. Wolf, C. Freeman, E. Wimmer, R. B. Adamson, L. Hallstadius, P. E. Cantonwine, and E. V. Mader, " $\mathrm{H}$ in $\alpha$-zr and in zirconium hydrides: solubility, effect on dimensional changes, and the role of defects," Journal of Physics: Condensed Matter, vol. 27, pp. 025402 1-12, 2015.

[24] M. Liyanage, R. E. Miller, and R. K. N. D. Rajapakse, "Multi-scale approach for determining hydrogen diffusivity in zirconium," Model. Simul. Mater. Sci. Eng., vol. 26, p. 085002, 2018.

[25] A. Denis, E. Moyano, and E. A. Garcia, "Model to simulate high temperature oxidation kinetics of zircaloy-4: Parabolic and linear behaviour," J. Nucl. Mater., vol. 110, pp. 11-19, 1982.

[26] R. Bordoni and A. Olmedo, "Oxide thickness measurements and characterization of two zr-2.5nb pressure tubes of embalse nuclear power plant," Acta Microsc., vol. 20, pp. 10-20, 2011.

[27] C. O. D. González and E. A. García, "Determination of the diffusion coefficients of oxygen in zirconium by means of xps," Applied Surface Science, vol. 44, pp. 211-219, 1990.

[28] C. J. Rosa and H. W. C., "Oxygen-ion diffusivity in hypostoichiometric zirconium dioxide," J. Nucl. Mater., vol. 27, pp. 12-20, 1968.

[29] L. Zhang, L.-Y. Chen, C. Zhao, Y. Liu, and Z. Lai-Chang, "Calculation of oxygen diffusion coefficients in oxide films formed on low-temperature annealed zr alloys and their related corrosion behavior," Metals., vol. 9, p. 850, 2019. 


\section{Chapter 5}

\section{Effect of Stress on Hydrogen Diffusion in Zirconium, investigated through multi-scale methods ${ }^{1}$}

In Chapter 4 we investigated a hypothesis proposing that $\mathrm{O}$ slows $\mathrm{H}$ diffusion near the surface of pressure tubes, which causes the formation of denuded zones. From this we found that even though $\mathrm{O}$ slows $\mathrm{H}$ diffusion it is not sufficient to cause a significant enough decrease in the size of the hydrides formed. This would mean that it could not be the main reason for the formation of denuded zones. Looking at the other hypotheses proposed (see Sec. 1.2.5) we now investigate whether the high tensile stresses near oxide-metal interface attracts the nearby hydrogen making a hydrogen depleted region closer to the surface. Most studies consider the effect of stress with the use of eqn. (5.1) where only the effect of the hydrostatic stress gradient is considered, while keeping diffusivity constant. In reality diffusivity depends on the stress and variation becomes more pronounced at high stress levels. We look at the effect of pressure (hydrostatic stress) on $\mathrm{H}$ diffusivity in $\mathrm{Zr}$, selecting a stress range that encompasses the stresses found in $\mathrm{Zr}$ closer to the metal oxide interface. We found that hydrostatic stress indeed affects the diffusivity of $\mathrm{H}$, where for tensile stresses diffusivity is increased and for compressive stresses it decreases. As future direction these results can be used in a study to look at how much $\mathrm{H}$ diffusion is affected by considering the changes to the diffusivity by high hydrostatic stresses.

\footnotetext{
${ }^{1}$ Contains the work in Manura Liyanage, Ronald Miller, and R. K. N. D. Rajapakse, "Effect of Stress on Hydrogen Diffusion in Zirconium, investigated through multi-scale methods", most work related to this publication is completed and expected to be submitted soon.
} 


\subsection{Introduction}

Formation of Hydride in Zr alloy vessels, which are used as pressure tubes containing the nuclear fuel in reactors [1-3], makes them susceptible to failure through mechanisms such as delayed hydride cracking (DHC) and loss of toughness. DHC occurs when hydrides accumulate in regions under tensile stresses, which causes the pressure tubes to fail before reaching its tensile strength [4-6]. Hence, continuous monitoring for the hydride content is carried out by taking scrape samples from the surface of the vessels to determine the suitability of keeping them in service. Hydride depleted zones or "denuded" zones found near the surface of some of these pressure tubes hinder the monitoring process as the surface hydride concentration will not accurately represent the bulk hydride content $[7,8]$. In our previous paper [9] we studied an idea which suggests that the high $\mathrm{O}$ concentration at the surface of the vessels causes the $\mathrm{H}$ to slow down, making it hard to form large enough hydride precipitates to be noticed during monitoring [7]. Though we found that $\mathrm{O}$ slows down the diffusion of $\mathrm{H}$ in $\mathrm{Zr}$, the $\mathrm{O}$ concentration over the thickness of the denuded zone is not sufficient to significantly decrease the size of the precipitates to form the denuded zones.

Another argument put forth is that an oxide layer usually present in the surface of the pressure tubes will cause a tensile stress at the oxide-metal interface, attracting $\mathrm{H}$ closest to it and depleting the $\mathrm{H}$ in the near-surface region. A counter argument for this mechanism is that $\mathrm{H}$ in the bulk will flow to the $\mathrm{H}$ depleted surface region due to the formed hydrogen gradient. This made us wonder how a stress gradient in $\mathrm{Zr}$ will affect the diffusivity of $\mathrm{H}$ and whether a change in the diffusivity caused by a stress gradient is the cause for the formation of denuded zones. Change in diffusion flux for $\mathrm{H}$ in a hydrostatic stress gradient shown as $[10,11]$,

$$
J=-D\left(\nabla C+\frac{C V}{k_{B} T} \nabla \sigma\right)
$$

where $J$ is the diffusion flux of $\mathrm{H}, C$ is the $\mathrm{H}$ concentration, $V$ is the partial molar volume of the solute hydrogen, $k_{B}$ is the Boltzmann constant, $T$ is the temperature, and $\sigma$ is the hydrostatic stress at the location. Here, the stress gradient acts as a driving force, making the diffusion equation diverge from the Fick's law.

In the current study we looked at how $\mathrm{H}$ in $\mathrm{Zr}$ is affected by hydrostatic stresses, 
while considering diffusivity to change with the hydrostatic stress. Here we used,

$$
J=-D_{\sigma} \nabla C
$$

since no driving or drifting force is applied. From the study we found that hydrostatic stress does indeed affect the diffusivity of $\mathrm{H}$. At tensile stresses we found an increase in the diffusivity while for compressive stresses the diffusivity decreases.

\subsection{Computational Method}

In two of our previous papers we used a multi-scale model, combining first principles calculations and Kinetic Monte Carlo simulations (KMC), to simulate hydrogen diffusion in $\alpha-\operatorname{Zr}$ [12] and $\alpha$-Zr with different concentrations of interstitial $\mathrm{O}$ [9]. For the current study we adopted the same model to simulate hydrogen diffusion in Zr under different hydrostatic stresses.

$\operatorname{Zr}(\alpha$-phase) has a hexagonal close packed unit cell with lattice parameters $a$ and $c$ (see Fig. 5.1), which effectively consists of two Zr atoms per unit cell. This crystal structure is anisotropic, though it shows isotropy in the AB planes which are called basal planes. $\mathrm{H}$ stably occupies two types of interstitial sites as shown in Fig. 5.2 namely tetrahedral sites and octahedral sites. H diffusion mainly happens through an interstitial mechanism where $\mathrm{H}$ atoms jump from one interstitial site to its nearest neighbor interstitial site.

We used the VASP code [13-15] to run first principles calculations, solving the many body Density Functional Theory (DFT) with the pseudopotential of projectoraugmented wave (PAW) method. The generalized gradient approximation of Perdew, Burke, and Ernzerhof (PBE-GGA) [16], which gives the best agreement to the lattice parameters of $\alpha-\mathrm{Zr}$, is used to estimate the exchange-correlation energies. $\mathrm{Zr}$ and $\mathrm{H}$ have valence configurations of $4 s^{2} 4 p^{6} 4 d^{2} 5 s^{2}$ and $1 s^{1}$ respectively. $4 \times 4 \times 4 \mathrm{Zr}$ supercells (containing $144 \mathrm{Zr}$ atoms) with periodic boundary conditions, plane wave cutoff energy of $400 \mathrm{eV}$, and K-point density of $5 \times 5 \times 5$ were used for all the first principles calculations, which gives good accuracy in simulations. The transition rate for $\mathrm{H}$ in the Zr lattice was found with Semi-Classical Harmonic Transition State Theory (SC-HTST) $[17,18]$, using the Climbing Image Nudged Elastic Band (NEB) 


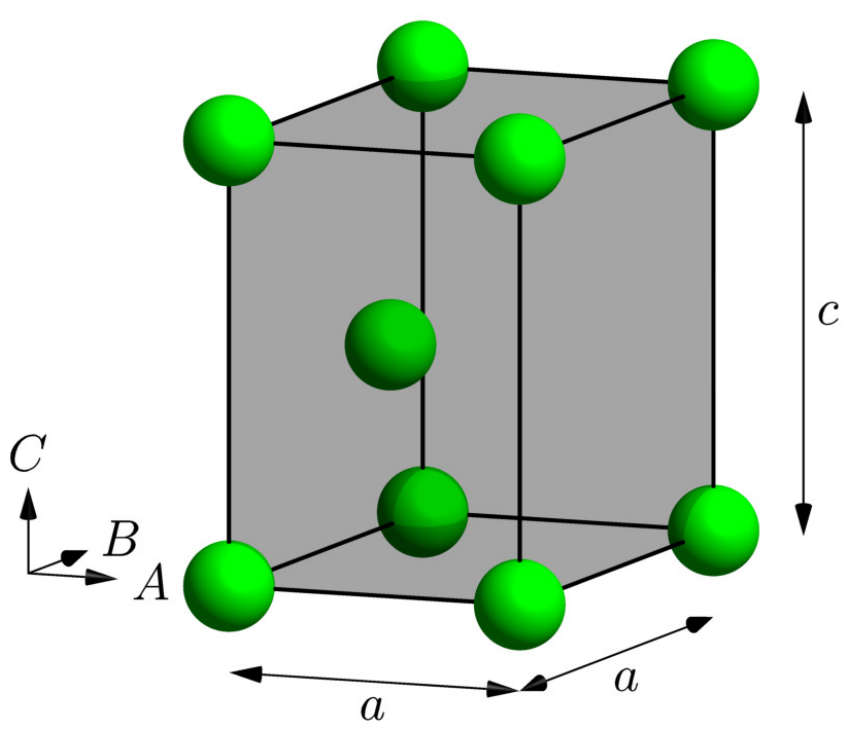

Figure 5.1: Unit cell for $\alpha \mathrm{Zr}$

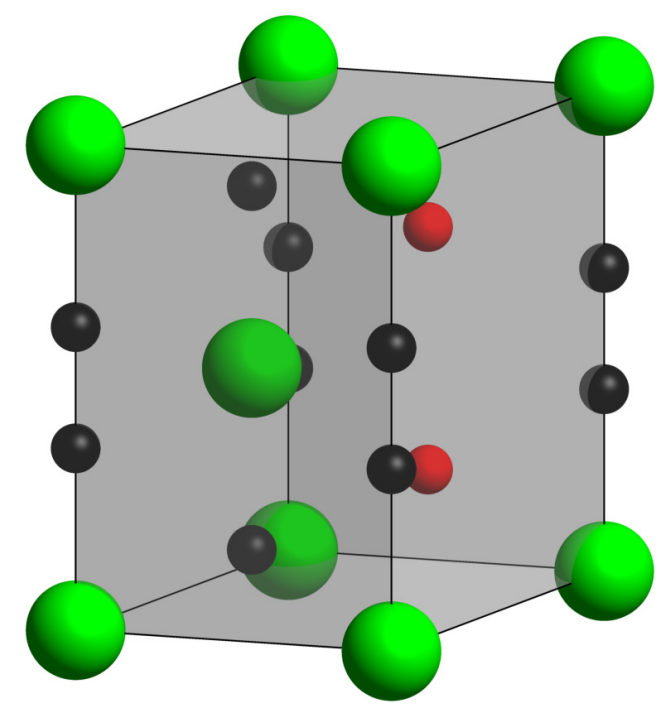

Figure 5.2: Locations available for $\mathrm{H}$ to occupy stably, where tetrahedral sites are shown in black colour spheres and octahedral sites are shown in red color. 
calculations [19] and central difference method, which includes the effects of Zero Point Energy (ZPE) and quantum tunneling. KMC simulations were carried out using inhouse code which was used successfully in our previous work [9,12]. Periodic boundary conditions were applied in the KMC simulations, which allowed us to simulate the motion of an $\mathrm{H}$ atom in a continuum by just using one unit cell.

Starting from a perfect $\mathrm{Zr}$ crystal we applied pressures (hydrostatic compressive stresses) ranging from -3000 to $2000 \mathrm{MPa}$ in steps of $1000 \mathrm{MPa}$ and the crystals were allowed to reach a relaxed configuration. Then we fixed the cell sizes for each case separately and added $\mathrm{H}$ atoms to a tetrahedral site and an octahedral site for each pressure to determine the binding energy of $\mathrm{H}$ for each site. In the $\mathrm{Zr}$ lattice $\mathrm{H}$ has four types of transitions between nearest neighbour sites; tetrahedral to tetrahedral, tetrahedral to octahedral, octahedral to tetrahedral, and octahedral to octahedral, which we represent by T-T, T-O, O-T, and O-O respectively (see Fig. 5.3). We used NEB calculations to identify the minimum energy paths for these transitons allowing us to find the hopping rates for each different case. These rates are then used in $\mathrm{KMC}$ simulations to estimate the diffusivity of $\mathrm{H}$ in $\mathrm{Zr}$ for temperatures between $100^{\circ} \mathrm{C}-300^{\circ} \mathrm{C}$. Using these we were able to identify the variation diffusivity of $\mathrm{H}$ in $\mathrm{Zr}$ for applied pressures. Thereafter we propose a model to extend the results from this study to simulate $\mathrm{H}$ diffusion in $\mathrm{Zr}$ for a known stress field.

\subsection{Results and Discussion}

\subsubsection{Variation of pressure}

As the initial step we used six cases with pressures varying from -3000 to $2000 \mathrm{MPa}$ in steps of $1000 \mathrm{MPa}$ to find the lattice parameters for $\mathrm{Zr}$ under these conditions. Cases are numbered in the order of increasing pressure starting from $-3000 \mathrm{MPa}$. Lattice parameters $(a$ and $c$ ) varies with the pressure as shown in Fig. 5.4, where for zero pressure we found $a=3.23 \AA$ and $c=5.17 \AA$ which agrees well with experimental values [20] and previous DFT work [21,22] available in literature. Lattice parameters follow a nearly linear variation within this pressure range. When $\mathrm{H}$ atoms are added to tetrahedral or octahedral sites we noticed an increase in the pressure of the cell. Fig. 5.5 shows the variation of pressure for systems with pure Zr, with $\mathrm{H}$ in a tetrahedral sites, and with $\mathrm{H}$ in an octahedral site for each different case. We can 


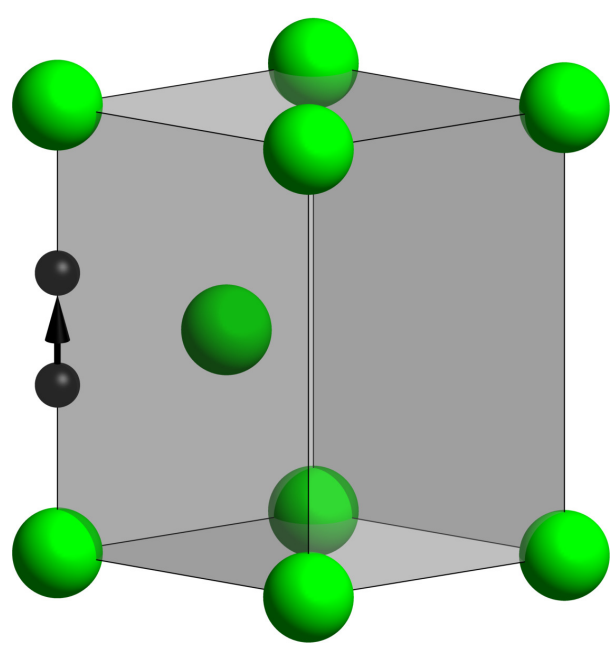

(a) $\mathrm{T}-\mathrm{T}$

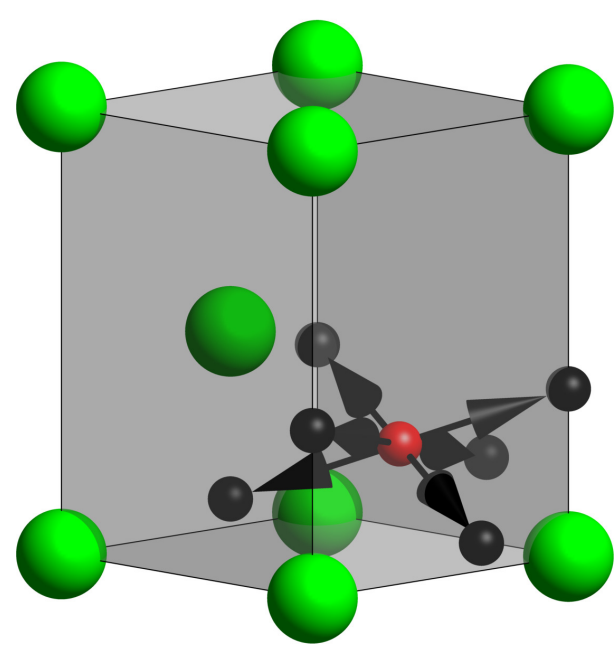

(c) $\mathrm{O}-\mathrm{T}$

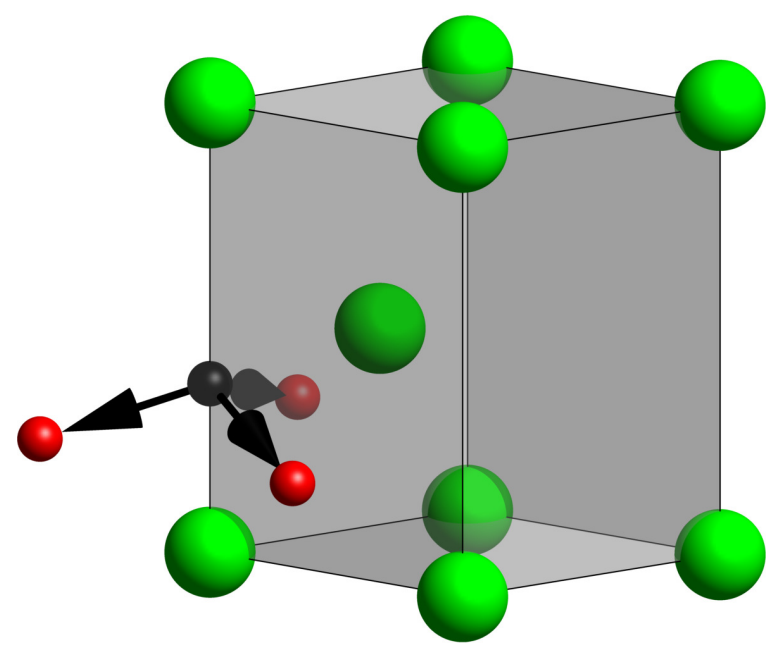

(b) $\mathrm{T}-\mathrm{O}$

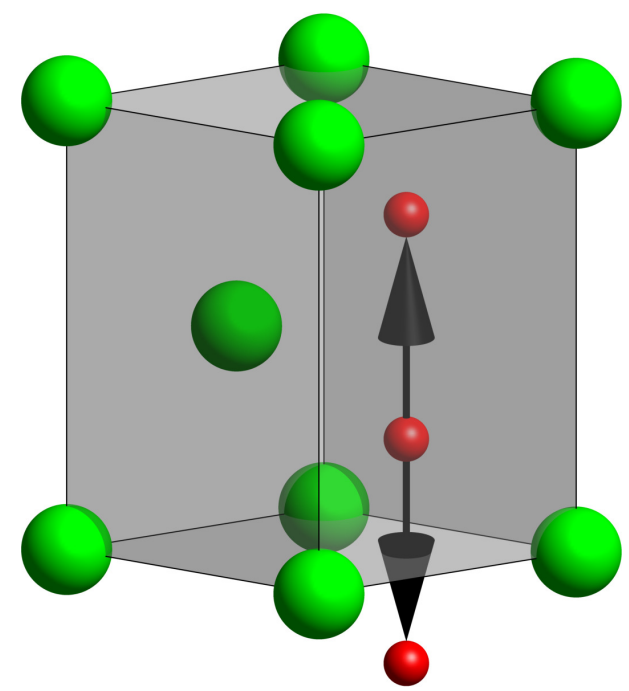

(d) $\mathrm{O}-\mathrm{O}$

Figure 5.3: The transition steps available from each interstitial site. The interstitial sites are represented by the same colors as in Fig. 5.2 


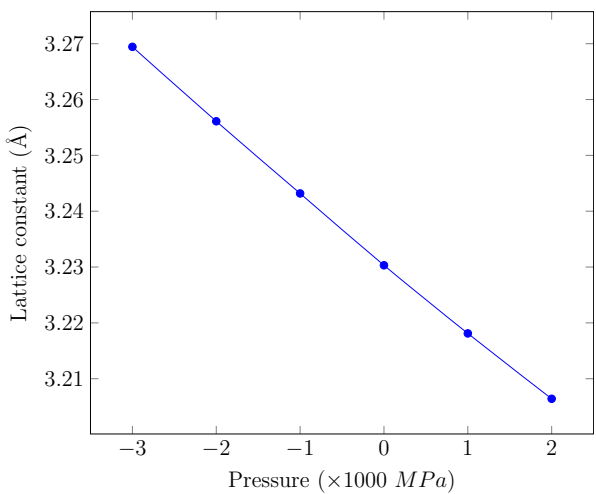

(a) Lattice parameter $a$

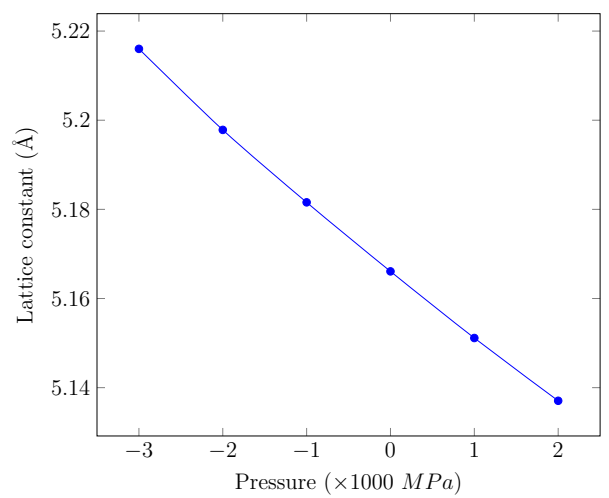

(b) Lattice parameter $c$

Figure 5.4: Variation of lattice parameters against pressure for $\alpha \mathrm{Zr}$

see a significant increase in the pressure when $\mathrm{H}$ atoms are added to $\mathrm{Zr}$ systems. The change in pressure for a tetrahedral sites shows a slightly higher value compared to the octahedral value. As the pressure in the pure Zr system increases, the additional pressure of adding a $\mathrm{H}$ atoms increase by a higher margin. Difference in pressure when adding a $\mathrm{H}$ atoms to a tetrahedral and octahedral sites is comparatively small. Hence, we used the average pressure of $\mathrm{H}$ occupying a tetrahedral and an octahedral site as the pressure of each case hereafter. The average pressure values for each case is tabulated in Table 5.1 compared to the pressure in pure Zr system. This pressure range was selected by looking at the stresses recorded close to the oxide metal interface in pressure tubes. Some studies have found that oxides formed on the zirconium alloys are under high compressive stresses around 1600-2500 MPa [23-25]. Hence we selected pressures shown in table 5.1, which encompasses these stresses.

\subsubsection{First principles calculations}

When there is no pressure acting on the systems, $\mathrm{H}$ atoms have a slight energy preference for the tetrahedral sites. For each of the different pressure, we looked at the change in preference for $\mathrm{H}$ occupancy among tetrahedral sites and octahedral sites as shown in Fig. 5.6. The preference for the tetrahedral site at 0 pressure shows a value of $0.06 \mathrm{eV}$, which agrees well with previous values ranging between 0.057 and $0.086 \mathrm{eV}$ [9,26-28]. Within this pressure range $\mathrm{H}$ sites still shows preference to 


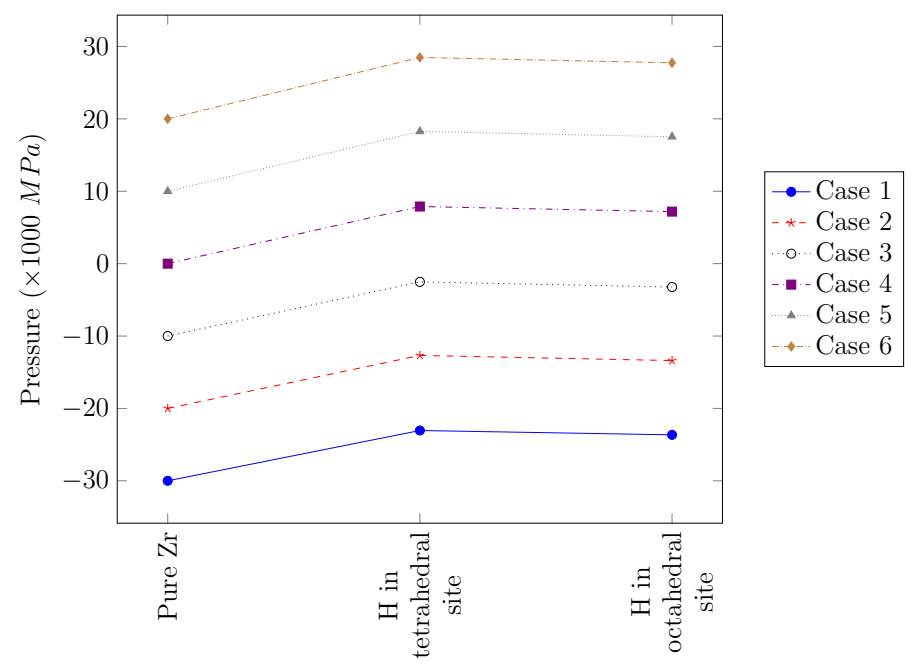

Figure 5.5: Change in pressure for pure $\mathrm{Zr}$, and systems with $\mathrm{H}$ in a tetrahedral or an octahedral site

Table 5.1: Average pressure in systems with $\mathrm{H}$ used in the study

\begin{tabular}{ccc}
\hline Case & Pressure in pure $\mathrm{Zr}(M P a)$ & Average pressure for systems with H $(M P a)$ \\
\hline \hline Case 1 & -3000 & -2335 \\
Case 2 & -2000 & -1304 \\
Case 3 & -1000 & -288 \\
Case 4 & 0 & 754 \\
Case 5 & 1000 & 1789 \\
Case 6 & 2000 & 2811 \\
\hline
\end{tabular}




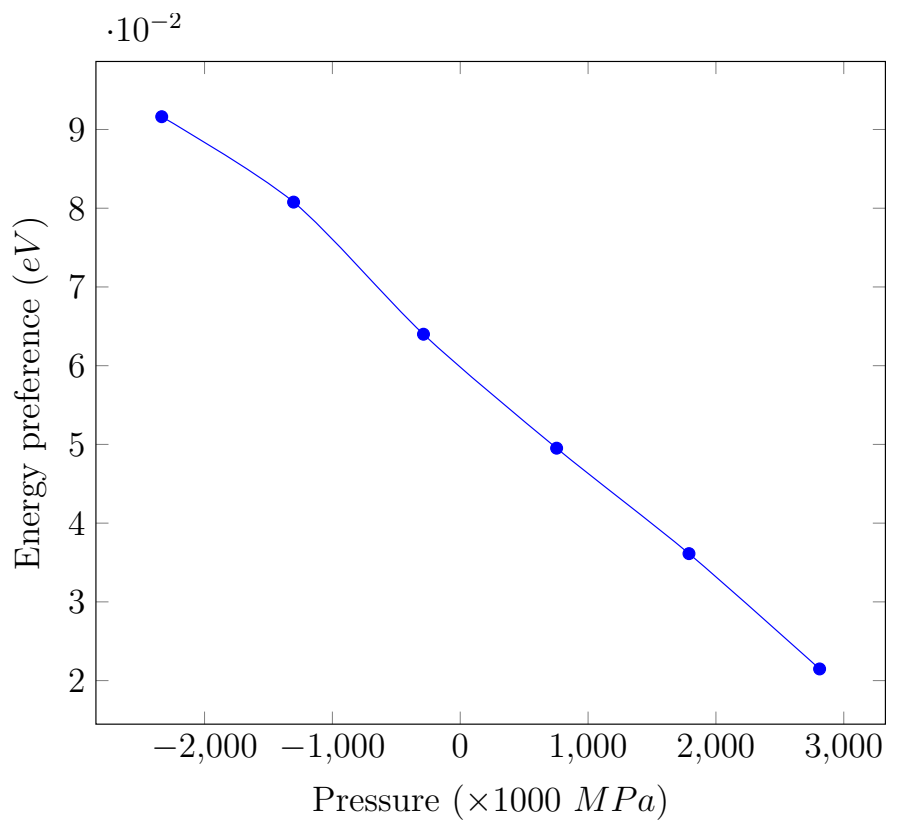

Figure 5.6: Variation of energy preference for tetrahedral sites against octahedral sites against the pressure of the system

tetrahedral sites, while the energy preference decreases as the pressure increases. The tetrahedral site has a smaller volume for H occupancy and smaller distances between the $\mathrm{H}$ and the $\mathrm{Zr}$ atoms. When the cell undergoes high pressures, the distance between $\mathrm{H}$ and the $\mathrm{Zr}$ atoms will decrease, while increasing the repelling forces between the atoms, making the site less stable. Octahedral sites have a higher volume, which will be more stable as there is enough space to contract with the pressures which explains the increased energy preference to it.

As identified for Zr, H diffusion occurs through an interstitial mechanism by jumping from an interstitial sites to one of its nearest neighbor sites. Using NEB calculations, we determined the activation energy of the minimum energy path for each distinct transition separately. Variations of the activation energy for each different transition against the pressure of the system are as shown in Fig. 5.7 and Fig. 5.8 below. For all the transition types we can see an increase in the activation energy when the applied pressure increases. The variation is much higher for the last two type of transitions, which signifies the transitions away from octahedral sites. This can be explained via the increased preference for the octahedral sites (relative to tetrahedral sites) when pressure increases, making it difficult to move away from them. 


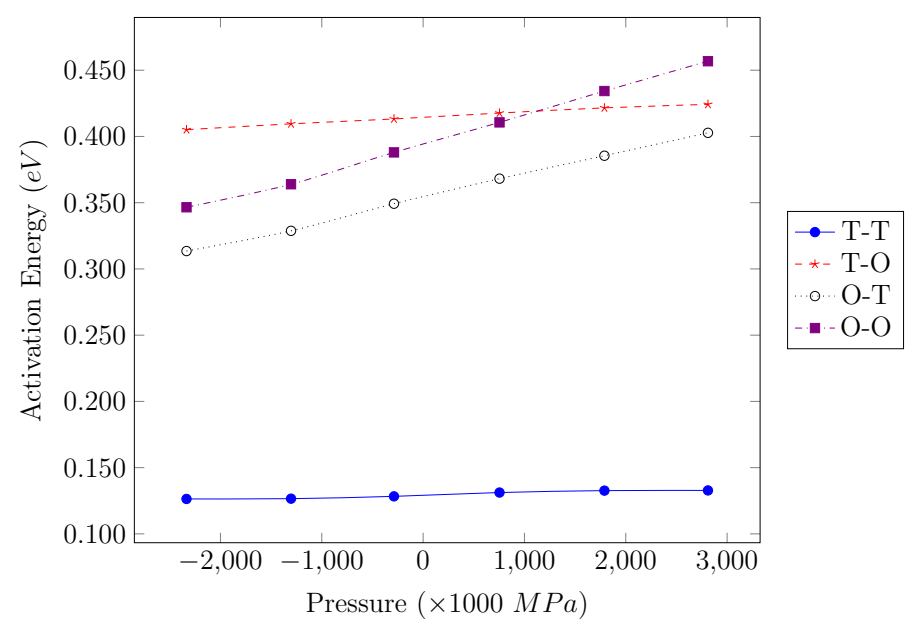

Figure 5.7: Variation of activation energy against pressure for all diffusion steps

Activation energy for T-T transitions does not vary linearly as the other transitions do and seems to converge as the pressure increases and decreases for this pressure range. From our study we didn't find any reasons which explains this variation, but this could be an interesting phenomenon to investigate and see whether this trend continues.

With the use of central difference method, the vibration frequencies for the interstitial sites and the identified saddle points for the minimum energy paths for each distinct transition were found. These values compare well with previous work of Zhang et al. [29] and Liyanage et al. [9]. The variation between the vibration frequencies with pressure is very small, where we see an increase in the frequencies for all modes when the pressure increases. With the use of activation energy and the vibration frequencies, the hopping rates for distinct transitions were determined though the SC-HTST through the methodology proposed in Puchala et al. [30] and Liyanage et al. [9] for temperatures between 100 and $300^{\circ} \mathrm{C}$ in steps of $50^{\circ} \mathrm{C}$. Transition between nearest neighbor tetrahedral sites (T-T) has a small activation energy compared to the other transitions, hence it acts as a pseudo-energy basin which will consume many of the transition steps in KMC simulations, while hopping back and forth between these sites, affecting the efficiency of our simulations. We got over this effect by considering this as an absorbing Markov chain allowing us to consider each pair of nearest neighbour tetrahedral sites as a single energy basin [9]. Modified hopping rates are then used in KMC simulations to determine the diffusivity of $\mathrm{H}$ in 


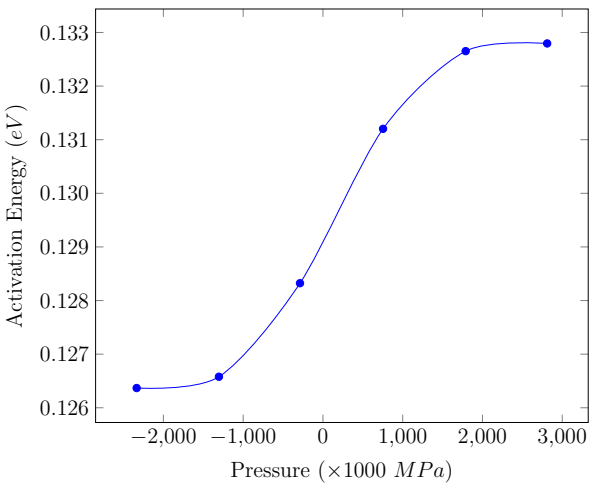

(a) T-T

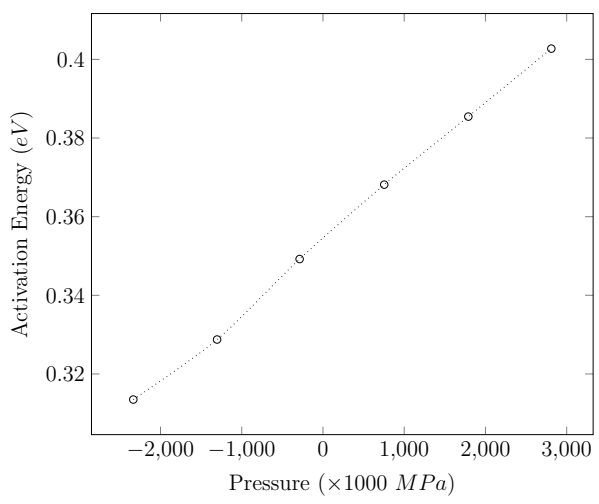

(c) $\mathrm{O}-\mathrm{T}$

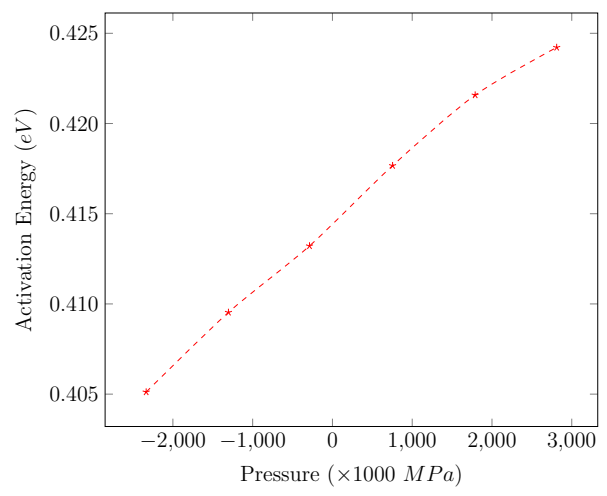

(b) $\mathrm{T}-\mathrm{O}$

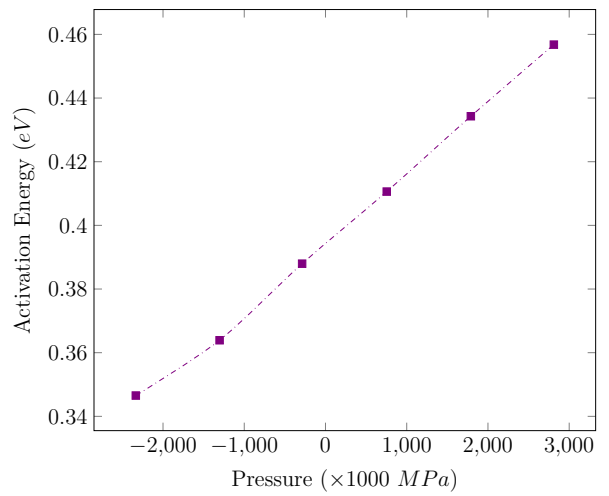

(d) $\mathrm{O}-\mathrm{O}$

Figure 5.8: Variation of activation energy against pressure for different transition steps 
Zr for different pressure levels.

\subsubsection{Diffusivity of $\mathrm{H}$ in $\mathrm{Zr}$}

The KMC simulations were carried out for the different pressure levels for the temperature range $100-300^{\circ} \mathrm{C}$ in steps of $50^{\circ} \mathrm{C}$. Fig. 5.9 shows the variation of the diffusivity against temperature for the different pressures. We can clearly see a reduction in the diffusivity when the pressure increases. If this variation is plotted as the logarithm of diffusivity $(\ln (D)$, where $D$ is the diffusivity) against the inverse of absolute temperature (1/T, where $T$ is the absolute temperature) (see Fig. 5.10) we see linear variations for all the cases, showing that the diffusion follows an Arrhenius form,

$$
D=D_{0} \exp \left(\frac{-E_{a}}{k_{B} T}\right)
$$

where $D$ is the diffusivity at temperature $T, E_{a}$ is the activation energy for diffusion, $D_{0}$ is the pre-exponent for diffusivity, and $k_{B}$ is the Boltzmann constant. Using the curves in Fig. 5.10, we obtained the activation energy $E_{a}$ and the pre-exponent $D_{0}$ for each of the cases found here. Fig. 5.11 shows the variation of $E_{a}$ with pressure for basal plane direction and $\mathrm{C}$-axis direction and Fig. 5.12 shows the variation of the pre-exponent $D_{0}$. We can see a clear increase in the activation energy with increase in pressure. $D_{0}$ doesn't show a significant variation as the pressure changes. As $E_{a}$ is the main parameter which affects the diffusion (since it comes in the exponent of the diffusivity equation) the small variation in the $D_{0}$ doesn't give a significant change to the change in diffusion.

Looking at the diffusivities in the two directions, basal plane direction and Caxis direction, in Fig. 5.9 we notice the diffusivity in c-axis direction shows a higher value, especially at high temperatures and low pressures. If we plot the anisotropy of diffusion $\left(D_{C} / D_{A B}\right.$, where $D_{C}$ and $D_{A B}$ are the diffusivities in C-axis and basal plane directions respectively) as shown in Fig. 5.13, we see that the anisotropy of diffusion increases with the temperature but decreases with the pressure. In our previous paper Liyanage et al. [12], we have discussed the reasons for the existence of the anisotropy in the diffusion process. There we identified TT-O and O-TT transitions to be the 


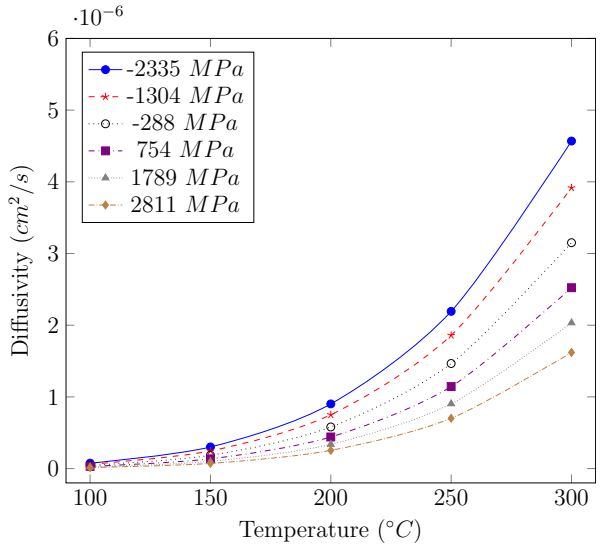

(a) Basal plane direction

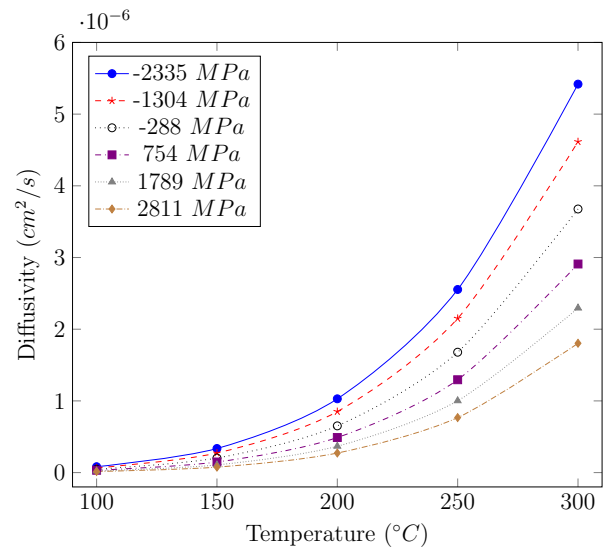

(b) C-axis direction

Figure 5.9: Variation of diffusivity against temperature for the different pressures

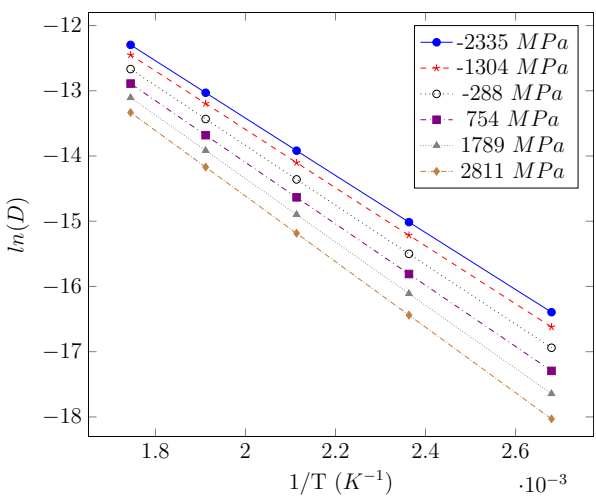

(a) Basal plane direction

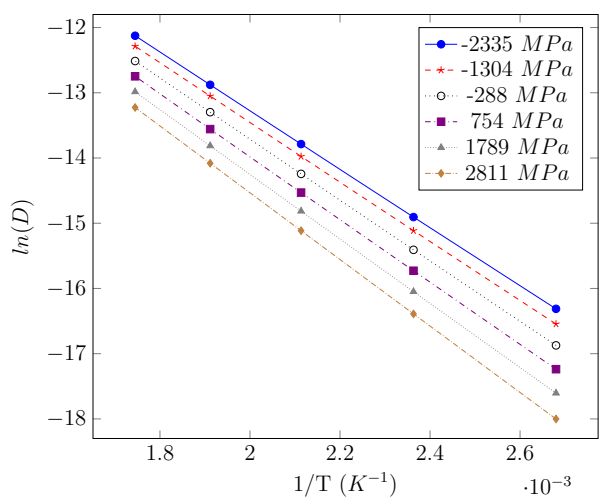

(b) C-axis direction

Figure 5.10: Variation of $\ln (D)$ against $1 / T$ for the different pressures 


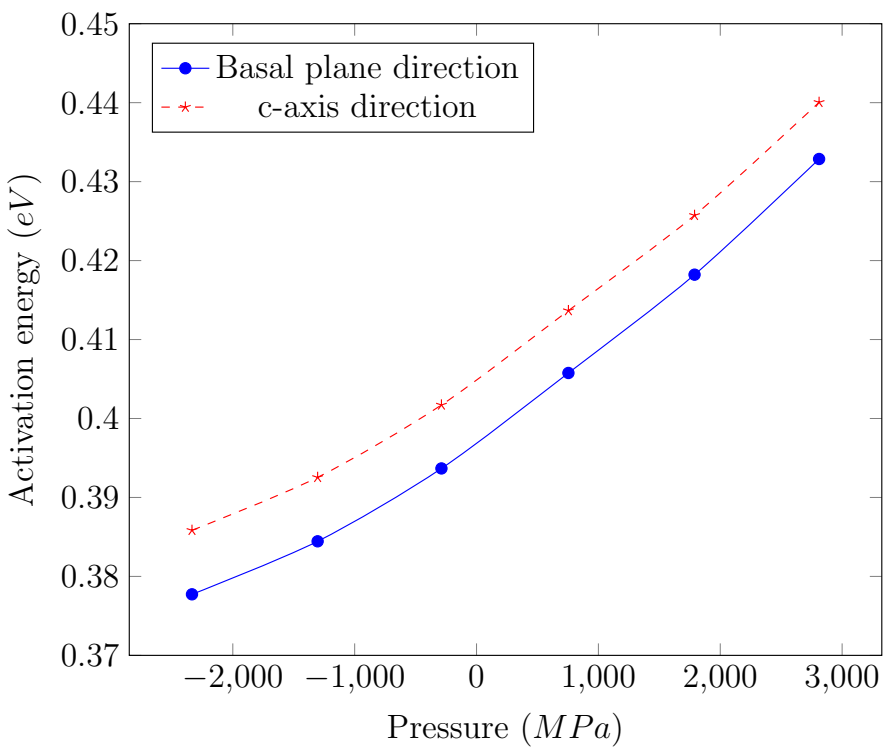

Figure 5.11: Variation of activation energy $E_{a}$ with the pressure

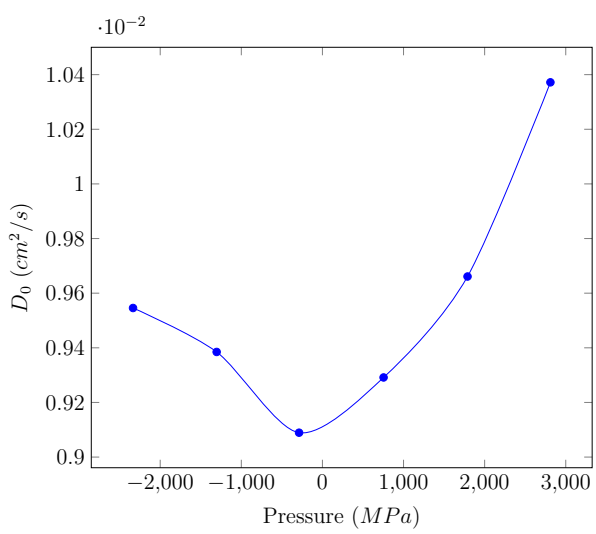

(a) Basal plane direction

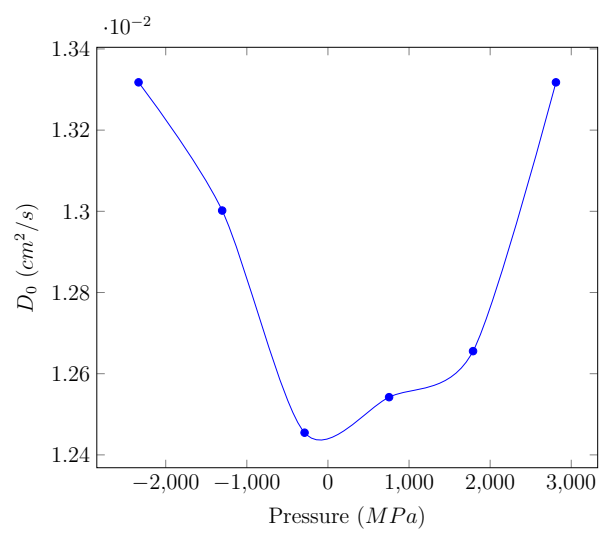

(b) C-axis direction

Figure 5.12: Variation of pre-exponent $D_{0}$ with the pressure 


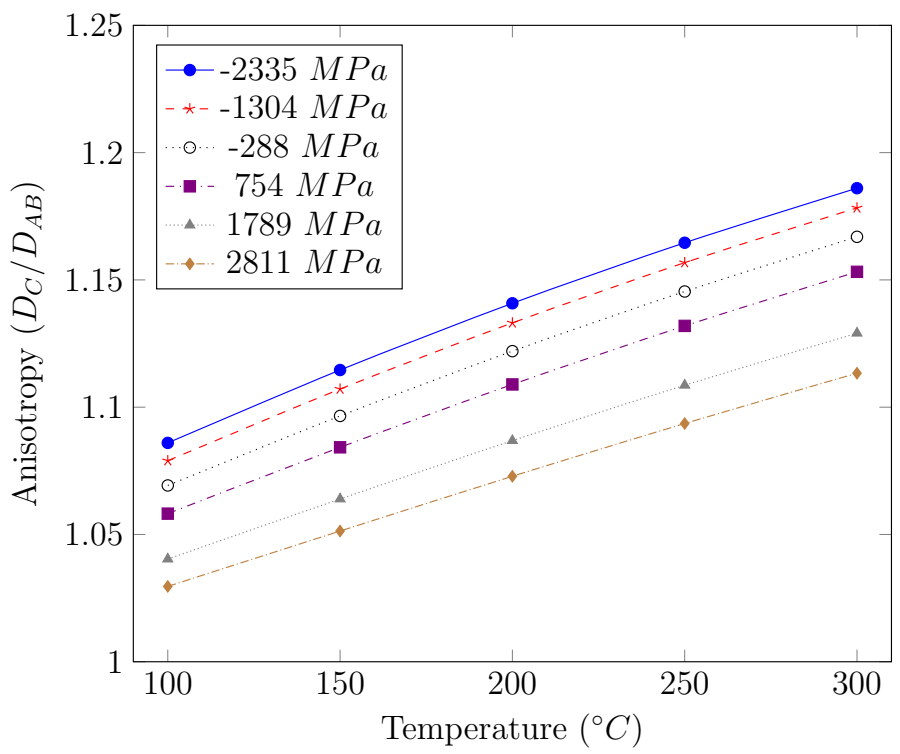

Figure 5.13: Variation of anisotropy $D_{C} / D_{A B}$ with temperature for different pressures

mains transition steps that contribute to the diffusion of $\mathrm{H}$ in $\mathrm{Zr}$ (TT represent the pseudo energy basin formed by nearest neighbour tetrahedral sites). The mean square displacements of these two steps have similar values, with a slightly higher value in the basal plane direction. The remaining transitions are O-O transitions, which occurs at a lower probability and forces the diffusivity in the $\mathrm{C}$-axis direction to increase without contributing to the diffusivity in the basal plane direction. The relative rate at which $\mathrm{O}-\mathrm{O}$ transitions occur is the main factor which determines the anisotropy for $\mathrm{H}$ in the $\mathrm{Zr}$ lattice. From Fig. 5.8(d) we can see that the activation energy of the O-O transition increases at a much higher rates than that of $\mathrm{T}-\mathrm{O}$ or the O-T transitions. This would mean that the contribution of the $\mathrm{O}-\mathrm{O}$ will decrease with the pressure causing the diffusivity in the $\mathrm{C}$-axis direction to reduce compared to the basal plane direction. This will make the anisotropy decrease when the pressure increase as seen in Fig. 5.13.

\subsection{Conclusions and Future Directions}

With the used of first principles calculations we were able to estimate the hopping rates for the different transitions steps for $\mathrm{H}$ diffusion in $\mathrm{Zr}$, when they are under 
different pressures. Using these in $\mathrm{KMC}$ simulations we determined the variation of $\mathrm{H}$ diffusivity for varying temperature and pressures. The diffusivity follows an Arrhenius form for the variation against the temperature. High pressures in $\mathrm{Zr}$ causes the diffusivity of $\mathrm{H}$ to decrease, while low pressures (tensile hydrostatic stresses) will cause the diffusivity of $\mathrm{H}$ to increase, mainly due to the increased stability of $\mathrm{H}$ occupancy in octahedral sites for high pressures.

While this study shows us that diffusivity is affected by stress, the situations for which the variation needs to be considered is not identified. Future direction would be to further extend this study to look at some scenarios for which $\mathrm{Zr}$ is under high hydrostatic stresses (about $1 \mathrm{GPa}$ ) and check the influence of including variation of diffusivity in the simulations.

\section{Bibliography}

[1] R. Krishnan and M. K. Asundi, "Zirconium alloys in nuclear technology," in Proceedings of the Indian Academy of Sciences Section C: Engineering Sciences, vol. 4, pp. 41-56, 1981.

[2] S. Banerjee, "Nuclear applications: Zirconium alloys," in Encyclopedia of Materials: Science and Technology (K. Buschow, R. Cahn, M. Flemings, B. Ilschner, E. Kramer, S. Mahajan, and P. Veyssiere, eds.), pp. 6287-6299, Elsevier, 2 ed., 2001

[3] D. Féron, "Overview of nuclear materials and nuclear corrosion science and engineering," in Nuclear Corrosion Science and Engineering (D. Féron, ed.), ch. 2, pp. 31-56, Woodhead Publishing, 2012.

[4] G. McRae, C. Coleman, and B. Leitch, "The first step for delayed hydride cracking in zirconium alloys," J. Nucl. Mater., vol. 396, pp. 130-143, 2010.

[5] M. De Las Heras, S. Parodi, L. Ponzoni, J. Mieza, S. Müller, S. Alcantar, and G. Domizzi, "Effect of thermal cycles on delayed hydride cracking in $\mathrm{Zr}-2.5 \mathrm{Nb}$ alloy," J. Nucl. Mater., vol. 509, pp. 600-612, 2018.

[6] T. N. Murty, R. Singh, and P. Ståhle, "Delayed hydride cracking of Zr-2.5\%nb pressure tube material due to partially constrained precipitates," J. Nucl. Mater., vol. 513, pp. 129-142, 2019.

[7] D. Hardie, "The effect of oxygen on the precipitation of hydrogen from zirconium," Journal of Nuclear Materials, vol. 17, pp. 88-90, 1965.

[8] C. Roy, "An experiment to clarify the effect of dissolved oxygen on the terminal solubility of hydrogen in zirconium," Journal of Nuclear Materials, vol. 13, pp. 275-277, 1964. 
[9] M. Liyanage, R. E. Miller, and R. K. N. D. Rajapakse, "Denuded zones in zirconium pressure vessels: Oxygen's role examined via multi-scale diffusion model." Manuscript submitted for publication, 2020.

[10] G. McRae, C. Coleman, H. Nordin, B. Leitch, and S. Hanlon, "Diffusivity of hydrogen isotopes in the alpha phase of zirconium alloys interpreted with the Einstein flux equation," Journal of Nuclear Materials, vol. 510, pp. 337-347, 2018.

[11] P. Sofronis and R. McMeeking, "Numerical analysis of hydrogen transport near a blunting crack tip," Journal of the Mechanics and Physics of Solids, vol. 37, no. 3 , pp. $317-350,1989$.

[12] M. Liyanage, R. E. Miller, and R. K. N. D. Rajapakse, "Multi-scale approach for determining hydrogen diffusivity in zirconium," Model. Simul. Mater. Sci. Eng., vol. 26, p. 085002, 2018.

[13] G. Kresse and J. Hafner, "Ab initio molecular dynamics for liquid metals," Physical Review B, vol. 47, no. 1, pp. 558-561, 1993.

[14] G. Kresse and J. Furthmüller, "Efficiency of ab-initio total energy calculations for metals and semiconductors using a plane-wave basis set," Computational Materials Science, vol. 6, no. 1, pp. 15-50, 1996.

[15] G. Kresse and J. Furthmüller, "Efficient iterative schemes for $<\mathrm{i}>$ ab initio $</ \mathrm{i}>$ total-energy calculations using a plane-wave basis set," Physical Review B, vol. 54, no. 16, pp. 11169-11186, 1996.

[16] J. P. Perdew, K. Burke, and M. Ernzerhof, "Generalized Gradient Approximation Made Simple," Physical Review Letters, vol. 77, no. 18, pp. 3865-3868, 1996.

[17] J. T. Fermann and S. Auerbach, "Modeling proton mobility in acidic zeolite clusters: II. room temperature tunneling effects from semiclassical rate theory," J. Chem. Phys., vol. 112, pp. 6787-6794, 2000.

[18] B. Bhatia and D. S. Sholl, "Quantitative assessment of hydrogen diffusion by activated hopping and quantum tunneling in ordered intermetallics," Phys. Rev. $B$, vol. 72, p. 224302, 2005.

[19] G. Henkelman and H. Jónsson, "Improved tangent estimate in the nudged elastic band method for finding minimum energy paths and saddle points Improved tangent estimate in the nudged elastic band method for finding minimum energy paths and saddle points," Journal of Chemical Physics, vol. 113, pp. 9978-9985, 2000 .

[20] E. S. Fisher and C. J. Renkin, "Single-crystal elastic moduli and the hcp - bcc transformation in ti, zr, and hf," Physical Review, vol. 135, no. 2A, pp. A482A494, 1964.

[21] F. Willaime, "Ab initio study of self-interstitials in hcp-Zr," Journal of Nuclear Materials, vol. 323, pp. 205-212, 2003. 
[22] G. Vérité, F. Willaime, and C. C. Fu, "Anisotropy of the vacancy migration in $\mathrm{Ti}, \mathrm{Zr}$ and Hf hexagonal close-packed metals from first principles," Solid State Phenomena, vol. 129, pp. 75-81, 2007.

[23] Y.-S. Kim, Y.-H. Jeong, and J.-N. Jang, "Stress measurements during thin film zirconium oxide growth," Journal of Nuclear Materials, vol. 412, pp. 217-220, 2011.

[24] E. Polatidis, P. Frankel, J. Wei, M. Klaus, R. Comstock, A. Ambard, S. Lyon, R. Cottis, and M. Preuss, "Residual stresses and tetragonal phase fraction characterisation of corrosion tested zircaloy-4 using energy dispersive synchrotron x-ray diffraction," Journal of Nuclear Materials, vol. 432, no. 1, pp. $102-112$, 2013.

[25] P. Platt, E. Polatidis, P. Frankel, M. Klaus, M. Gass, R. Howells, and M. Preuss, "A study into stress relaxation in oxides formed on zirconium alloys," Journal of Nuclear Materials, vol. 456, pp. 415 - 425, 2015.

[26] C. Domain, R. Besson, and A. Legris, "Atomic-scale ab-initio study of the zrh system: I. bulk properties," Acta Materialia, vol. 50, no. 13, pp. 3513-3526, 2002

[27] Y. Zhang, C. Jiang, and X. Bai, "Anisotropic hydrogen diffusion in $\alpha$-Zr and Zircaloy predicted by accelerated kinetic Monte Carlo simulations," Scientific Reports, vol. 7, pp. 41033 1-13, 2017.

[28] P. A. Burr, S. T. Murphy, S. C. Lumley, M. R. Wenman, and R. W. Grimes, "Hydrogen accommodation in zr second phase particles: implications for h pickup and hydriding of zircaloy-2 and zircaloy-4," Corrosion Science, vol. 69, pp. 14, 2013.

[29] L. Zhang, L.-Y. Chen, C. Zhao, Y. Liu, and Z. Lai-Chang, "Calculation of oxygen diffusion coefficients in oxide films formed on low-temperature annealed zr alloys and their related corrosion behavior," Metals., vol. 9, p. 850, 2019.

[30] B. Puchala, M. L. Falk, and K. Garikipati, "An energy basin finding algorithm for kinetic monte carlo acceleration," J. Chem. Phys., vol. 132, p. 134104, 2010. 


\section{Chapter 6}

\section{First-principles study of hydrogen in lead zirconate titanate $^{1}$}

Through the developed model we have mainly simulated $\mathrm{H}$ diffusion in the Zr lattice. While looking at some other materials, we noticed lead zirconate titanate (PZT), which is a popular piezoelectric material which also undergoes degradation with $\mathrm{H}$ absorption. PZT has a complicated crystal structure due to the spontaneous polarization at low temperatures, which gives it high piezoelectric properties. While $\mathrm{H}$ causes it to degrade there are very few studies which looks at the $\mathrm{H}$ diffusion in PZT, which is an important property for investigating $\mathrm{H}$ based degradation. So this made us look at the behavior of $\mathrm{H}$ in PZT with the use of first principles calculations. As an initial step we found the parameters to model tetragonal PZT crystals (the most common phase found in room temperatures). As the next step we looked at possible locations where $\mathrm{H}$ can occupy stably in the PZT lattice. We found 5 sites which can be occupied by $\mathrm{H}$ stably, which matched with the identified locations in $\mathrm{PbTiO}_{3}$ (another piezoelectric material with similar stucture). Then we looked at the effect of $\mathrm{H}$ occupancy in these locations on the polarization of the structure, which is the main problem PZT faces due to $\mathrm{H}$ based degradation. Thereafter we propose transition paths available for escaping from each occupancy location to reach a nearest neighbour site. In the future we will carry out further calculations on this to determine the diffusion coefficients for $\mathrm{H}$ diffusion in PZT.

\footnotetext{
${ }^{1}$ Contains the work in Manura Liyanage, Ronald Miller, and R. K. N. D. Rajapakse, "Firstprinciples study of hydrogen in lead zirconate titanate", Smart Materials and Structures, vol. 28, no. 3, pp. 034002 1-11, 2019.
} 


\subsection{Introduction}

\subsubsection{Motivation of the study}

Lead zirconate titanate (PZT) is a perovskite crystal $\left(\mathrm{ABO}_{3}\right.$ structure), with $\mathrm{PbZr}_{x} \mathrm{Ti}_{1-x} \mathrm{O}_{3}$ chemical formula, which is known for its excellent ferroelectric, piezoelectric and dielectric properties. PZT is used or proposed to be used in a large number of applications [1]. Hydrogen technology is currently a major research area in clean energy engineering. In recent decades, substantial progress has been made in the development of fuel injectors which use piezoelectric actuators for valve opening [2]. This piezo-actuator-based technology has many advantages over the conventional solenoid-based injectors such as excellent fuel metering, better valve opening control, high injection pressure, rapid response, excellent repeatability, and low power consumption. As a result, there is industrial interest to use piezo-actuator-based technology to develop hydrogen fuel injectors for clean energy production and applications. It is widely known that hydrogen absorption causes a loss of ductility and premature failure in metals [3-5]. Similarly, PZT is also adversely affected by hydrogen, which can cause degradation to PZT's electric properties, cracking, and fracture. Therefore, the application of piezo-actuator-based injectors in hydrogen technology requires further research.

Hydrogen in PZT is a widely studied topic in the research community, but diffusion of hydrogen in PZT is an area which has attracted very little of that attention. An understanding of the diffusion behaviour of hydrogen in PZT is fundamentally important to hydrogen technology applications. An approach to determine diffusivity of hydrogen in the hexagonal close packed zirconium was presented in one of our previous papers [6], which uses a multi-scale approach with first principles calculations and Kinetic Monte Carlo (KMC) simulations. The current paper contains the initial results of the study that aims to extend the above-mentioned approach to study hydrogen diffusion in tetragonal PZT. In this paper, we have identified the occupancy locations of hydrogen atoms in the verified tetragonal PZT crystal structure using first-principles Density Functional Theory (DFT) calculations. We have also determined the effect on the dimensions, volume, and polarity of the crystal when hydrogen occupy each of these locations. As well, we have proposed possible paths available for hydrogen to escape from each of the identified occupancy locations. These transition steps will be the steps of the diffusion paths that hydrogen will use to travel through 
the lattice.

Results from the current study will be extended to identify the rate constants for the identified diffusion steps, which we will then be using as input to KMC simulations to estimate diffusivity of hydrogen in the tetragonal PZT crystal in our future studies.

\subsubsection{Lead zirconate titanate (PZT)}

PZT is a solid solution of $\mathrm{PbZrO}_{3}(\mathrm{PZ})$ and $\mathrm{PbTiO}_{3}(\mathrm{PT})$ in varying ratios, having a crystal structure made up of $\mathrm{ABO}_{3}$ type unit cells (see Fig. 6.1). In PZT, A is $\mathrm{Pb}$ and $\mathrm{B}$ is either $\mathrm{Zr}$ or $\mathrm{Ti}$ in distributed in a random order. Due to the spontaneous polarization caused by displacement of $\mathrm{Zr}^{4+}$ and $\mathrm{Ti}^{4+}$ atoms at low temperatures (see Fig. 6.1(b)) PZT has a polarization hysteresis behavior (see Fig. 6.2) which classifies it as a ferroelectric material. PZT has many advantageous properties such as high piezo electric charge coefficient, piezo electric voltage coefficient, high dielectric constant, high Curie temperature, high electro-mechanical coupling coefficient, high charge sensitivity, high energy density, good optical properties, radiation resistance, rapid response, and low mechanical loss [1,7-10]. These properties make it appealing for many applications including ultrasonic transducers, ceramic capacitors, actuators - used in hydrogen fuel injectors, sensors, diagnostic probes, micro-electromechanical systems, storage devices, ferroelectric random-access memories (FeRAM), antireflective coating, electro-optical modulators, space applications, and transformers [1,7-17].

PZT has a complex phase diagram which depends on temperature and PZ:PT ratio ( $x: 1-x$, where $x$ is the proportion of $\mathrm{PZ}$ in the mix) as shown in Fig. 6.3. In the figure $\mathrm{P}_{C}$ refers to the cubic structure (see Fig. 6.1(a)) occurring at very high temperatures (above curie temperature) which does not have polarization hysteresis and shows a paraelectric behaviour. Low temperature rhombohedral and tetragonal crystal structures show ferroelectric behaviour, where with high PZ content, the structure will be rhombohedral $\left(\mathrm{F}_{R_{L T}}\right.$ and $\mathrm{F}_{R_{H T}}$ in Fig. 6.3) and with high PT content the structure will be tetragonal ( $\mathrm{F}_{T}$ in Fig. 6.3). Both PZ and PT $(x=0$ and $x=1)$ shows very weak ferroelectric behavior. Closer to $x=0.5$, PZT displays its highest ferroelectric, piezoelectric, and dielectric properties. This is the boundary between the tetragonal and rhombohedral crystal structures called the morphotropic phase boundary (MPB) $[1,7,19,20]$. In recent studies, it has been found that this is not 


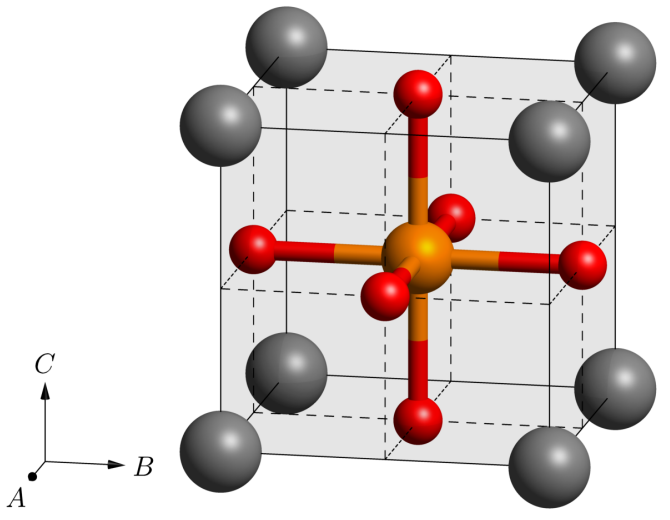

(a) Cubic structure

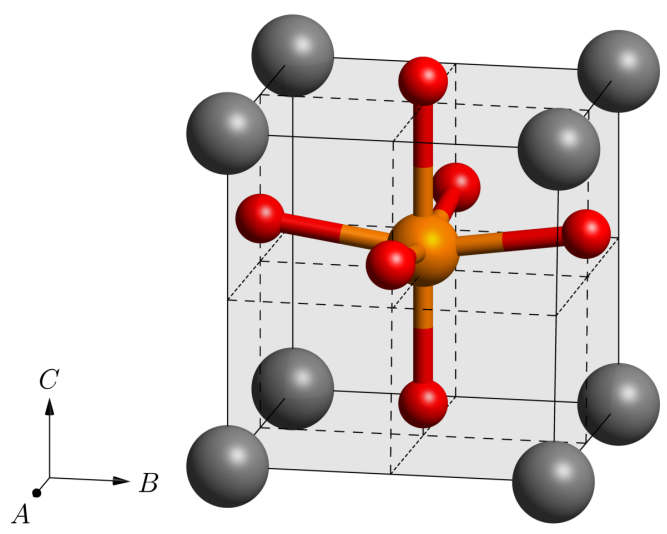

(b) Tetragonal structure

Figure 6.1: Arrangement of atoms in a PZT unit cell with $\mathrm{ABO}_{3}$ arrangement, grey color spheres represent $\mathrm{Pb}$ atoms, orange colour spheres represent either $\mathrm{Zr}$ or Ti randomly distributed through the lattice $\left(\mathrm{B}\right.$ in a $\mathrm{ABO}_{3}$ unit cell) and red color spheres shows the $\mathrm{O}$ atoms

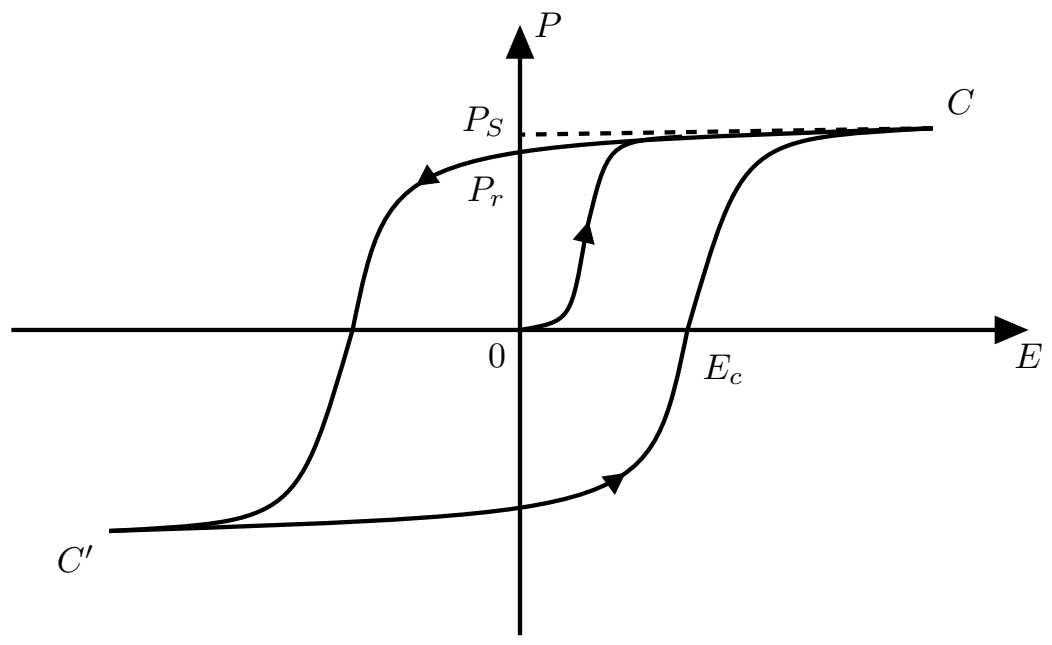

Figure 6.2: Polarization Hysteresis behavior of a ferroelectric material where, $\mathrm{P}_{s}$, spontaneous polarization. $\mathrm{P}_{r}$, remnant polarization. $\mathrm{E}_{c}$, coercive field [18] 


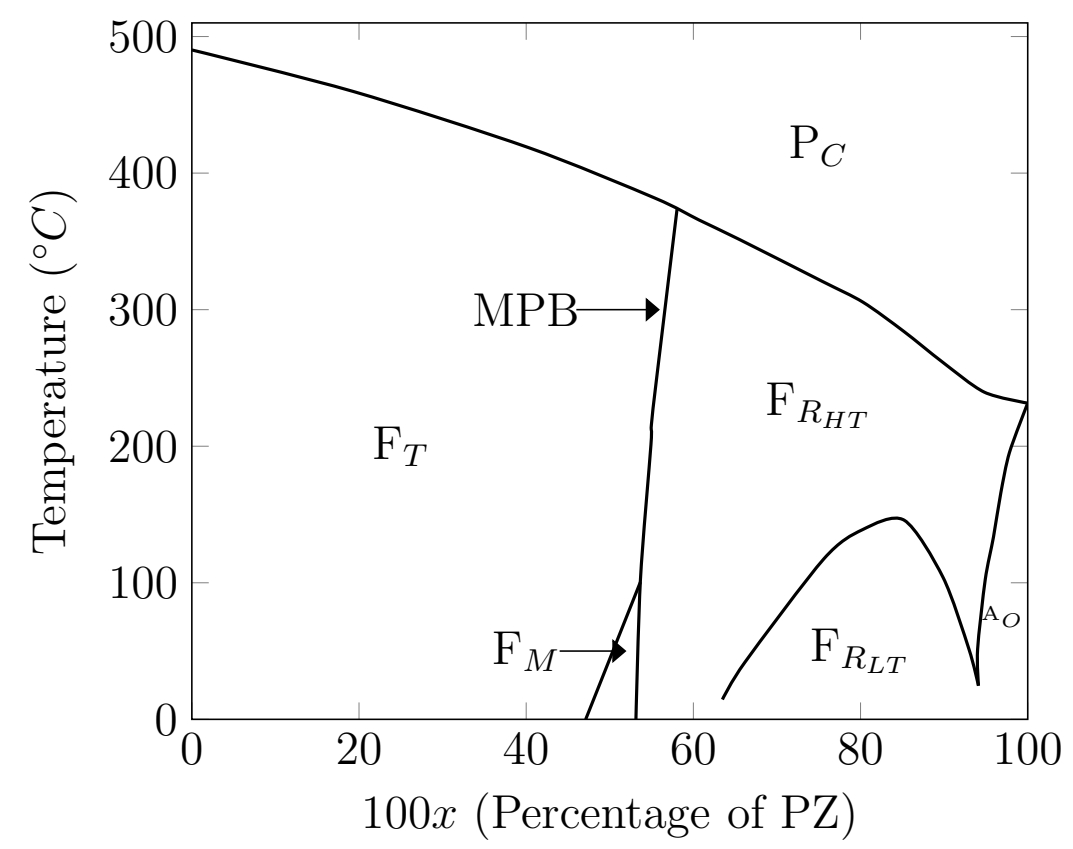

Figure 6.3: Phase diagram of PZT with respect to temperature and PZ:PT ratio, from Jaffe et al. [22] and Noheda et al. [21]

a clear boundary but a small region. Some studies suggest that in this region, both tetragonal and rhombohedral structures coexist. Recent studies suggest that a monoclinic phase, which has superior ferroelectric properties, exist in this region [20,21]. In the current study, we limit our study to $x=0.5\left(\mathrm{PbZr}_{0.5} \mathrm{Ti}_{0.5} \mathrm{O}_{3}\right)$ assuming it has a tetragonal crystal structure which has been used in several previous studies.

The high temperature cubic crystal structure is symmetric about all the orthogonal axis directions (see Fig. 6.1(a)), but in the tetragonal crystal structure it is elongated about the c-axis direction due to the polarization created by shifting of $\mathrm{Zr}^{4+}$ and $\mathrm{Ti}^{4+}$ ions and the octahedral oxygen cage around those ions (see Fig. 6.1(b)). The centers of the positive and the negative charges does not coincide which results in the polarisation. Table 6.1 below lattice parameters obtained through experimental and computational (first principles DFT calculations) methods in some previous studies. The fractional coordinates in C-direction for atoms in an $\mathrm{ABO}_{3}$ unit cell are shown in Table 6.2. These will be used to verify the model we will be using in this study. 
Table 6.1: Lattice parameters of tetragonal PZT unit cell $\left(\mathrm{ABO}_{3}\right)$ from previous studies (see Fig. 6.4)

\begin{tabular}{cccc}
\hline Reference & $a(\AA)$ & $c(\AA)$ & Method \\
\hline \hline Kitamura et al. [7] & 3.972 & 4.174 & DFT \\
Ribeiro et al. [8] & 4.007 & 4.239 & DFT \\
Shimakawa \& Kubo [16] & 4.029 & 4.149 & Experimental \\
Alvine et al. [11] & 4.050 & 4.110 & Experimental \\
Noheda et al. [20] & 4.037 & 4.138 & Experimental \\
Frantti et al. [23] & 4.030 & 4.144 & Experimental \\
Zhang et al. [24] & 4.032 & 4.148 & Experimental \\
Jia et al. [25] & 4.040 & 4.128 & Experimental \\
\hline
\end{tabular}

Table 6.2: Fractional c-direction coordinates in an $\mathrm{ABO}_{3}$ unit cell from previous studies (see Fig. 6.4)

\begin{tabular}{cccccc}
\hline Reference & $\mathrm{Pb}$ & $\mathrm{Zr} / \mathrm{Ti}$ & $\mathrm{O}_{1}$ & $\mathrm{O}_{2}$ & Method \\
\hline \hline Kitamura et al. [7] & 0.000 & 0.551 & 0.100 & 0.637 & $\mathrm{DFT}$ \\
Ribeiro et al. [8] & 0.000 & 0.549 & 0.110 & 0.637 & $\mathrm{DFT}$ \\
Sághi-szabó et al. [26] & 0.000 & 0.546 & 0.094 & 0.620 & DFT \\
Frantti et al. [23] & 0.000 & 0.565 & 0.092 & 0.611 & Experimental \\
Zhang et al. [24] & 0.000 & 0.569 & 0.044 & 0.524 & Experimental \\
\hline
\end{tabular}

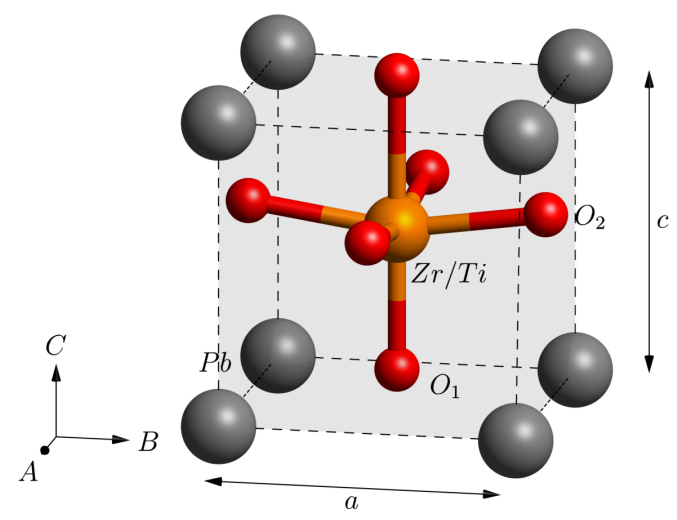

Figure 6.4: Arrangement of atoms in a tetragonal PZT unit cell 


\subsubsection{Hydrogen degradation of PZT}

While PZT has many advantageous properties, exposure to hydrogen has known to cause a wide array of problems, lossing some of its appeal in applications. Microelectronic devices are annealed in a mixture of $\mathrm{N}_{2}$ and $\mathrm{H}_{2}$ (about $4 \%$ of $\mathrm{H}_{2}$ in the mixture) at high temperatures to improve device quality $[27,28]$. During this charging process, it has been found hydrogen will enter PZT and diffuse inside the lattice [29]. Presence of electrodes which act as catalysts for $\mathrm{H}_{2}$ disassociation has been found to increase the amount of hydrogen absorbed [15,30-33]. This suggests that hydrogen will get absorbed and diffuse in the PZT lattice in its atomic form, similar to that of metals $[33,34]$. In actuators of HICE (Hydrogen Internal Combustion Engines), PZT gets exposed to high pressure $\mathrm{H}_{2}$ gas during operations [11].

The main issue of hydrogen exposure is the loss of the polarization hysteresis behavior in PZT [15, 16,31,32,35]. At low hydrogen contents, it has been found to improve the hysteresis behavior, which gives rise to the possibility of tuning the ferroelectric properties of PZT [27]. After the small range where the properties improve, hydrogen will cause the ferroelectric behavior to reduce and finally to disappear (see Fig. 6.5). Thereafter the behavior will be paraelectric similar to that of cubic PZT. Hydrogen absorption has also found to decrease the resistance in PZT, evident through observations of high leakage current [11,15,16,32,36,37]. The range of resistance has been found to decrease from $10^{12}-10^{13} \Omega \mathrm{cm}$ to $10^{6}-10^{7} \Omega \mathrm{cm}$ which causes PZT to loss its usefulness in capacitors, turning it from an insulator to a semiconductor [38]. In addition, hydrogen has also been found to cause PZT to fail physically as well. When charging, it has been observed that cracks have appeared in the PZT and the existing cracks have increase in size [29,34]. Surface blistering, delamination of electrodes, discoloration, delayed fracture, and embrittlement are some of the other modes of failure that has been observed [11, 29, 32, 39].

\subsubsection{Mechanisms of hydrogen degradation}

A few proposes mechanisms have been proposed to explain hydrogen degradation process of PZT. It has been found that hydrogen will mainly exist in $\mathrm{H}^{+}$form, forming covalent $\mathrm{O}-\mathrm{H}$ bonds with oxygen atoms. These hydroxyl groups are expected to reduce the polarization of the PZT lattice $[11,12,28,32,35]$. It has also been hypothesized to hinder the transformation from its high temperature cubic form, which would 


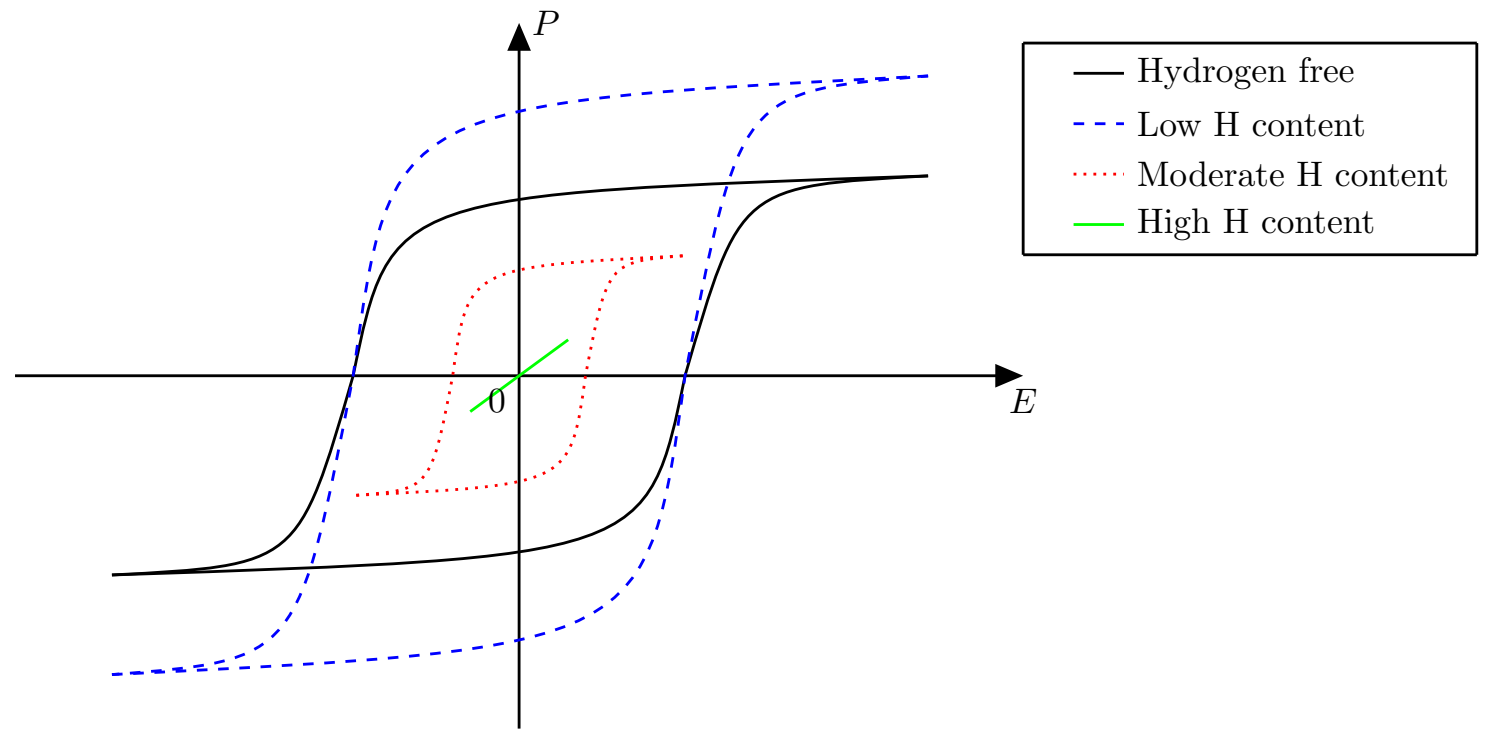

Figure 6.5: Hysteresis loops of the PZT ceramics with different trapped hydrogen concentrations [27]

explain the paraelectric behavior at high hydrogen content since annealing occur at high temperatures $[33,38]$. Electrons released during ionization of hydrogen and ionized vacancies created when hydrogen bonds with oxygen to form $\mathrm{H}_{2} \mathrm{O}$ molecules are set forwards as reasons for the reduction in resistance of PZT $[38,40]$. Reduction of $\mathrm{Pb}^{2+}$ ions to $\mathrm{Pb}^{0}$ atoms is another reason which is expected to transform PZT from an insulator to a semiconductor [36]. Color changes (from yellow to black) observed during hydrogen annealing, expected to be caused by $\mathrm{Pb}$ migrated to the surface and oxygen vacancies are used as proof for these hypotheses. It is also expected that hydrogen will get attracted to areas with non-uniform stress concentrations such as microcracks [29]. These hydrogen is expected to make $\mathrm{O}-\mathrm{H}$ bonds reducing the cohesive strength between oxygen and metallic atoms, which would cause the growth of available microcracks. It is also expected that hydrogen will diffuse to grain boundaries and microcrack surfaces and then combine to form $\mathrm{H}_{2}$ molecules. This could cause formation of cracks along grain boundaries and growth of existing cracks [34].

\subsubsection{Hydrogen diffusion and occupancy}

There are very few studies of the diffusion behavior of hydrogen in PZT, and their results are inconclusive. This motivates our own. Shafiei et al. [33] has observed an activation barrier of $0.442 \pm 0.1 \mathrm{eV}$ for hydrogen diffusion in cubic PZT. Alvine et 


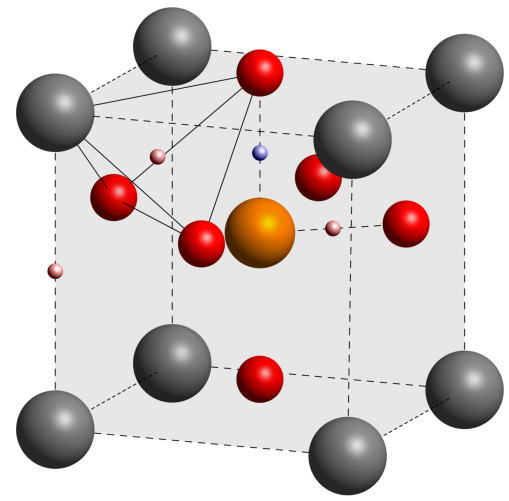

Figure 6.6: Possible locations for hydrogen occupancy as proposed by Aggarawal et al. [35]

al. [12] has observed that diffusivity coefficient $D$ has a range of $3-8 \times 10^{-10} \mathrm{~m}^{2} / \mathrm{s}$ in the temperature range $250 K-340 K$, which is considerably faster than the value obtained by Huang et al. [38] of $4.9 \times 10^{-12} \mathrm{~m}^{2} / \mathrm{s}$ at $288 \mathrm{~K}$.

Aggarawal et al. [35] has proposed four locations for hydrogen occupancy in PZT (see Fig. 6.6) based on Ramen spectroscopy results for a sample annealed in formation gas $\left(\mathrm{H}_{2}\right.$ and $\mathrm{N}_{2}$ gases $)$ at $450^{\circ} \mathrm{C}$. Small spheres indicate possible locations for $\mathrm{H}$ occupancy inside the PZT lattice structure and the one coloured blue is argued to be the most likely (see Fig. 6.6). It is also stated that hydrogen could only exist at this location in the cubic structure, and it is expected to hinder switching to the tetragonal phase. Alvine et al. [11] has proposed four locations for hydrogen occupancyfor the tetragonal PZT lattice based on previous studies for material with similar crystal structures such as $\mathrm{PbTiO}_{3}$ and $\mathrm{SrTiO}_{3}$ [41,42]. Small spheres indicated possible locations for $\mathrm{H}$ inside the PZT lattice structure as shown in Fig. 6.7. While this could be used as a guide for our study, we kept in mind that the variation of the B location in the perovskite structure as either $\mathrm{Zr}$ or Ti in PZT breaks some of the the symmetries available in the above-mentioned structures.

\subsection{Research Methodology}

As mentioned earlier, the final aim of the study is to model the diffusion behavior of hydrogen in the tetragonal PZT lattice, using the approach presented in one of our previous work [6]. In this paper we are presenting the results for the possible 


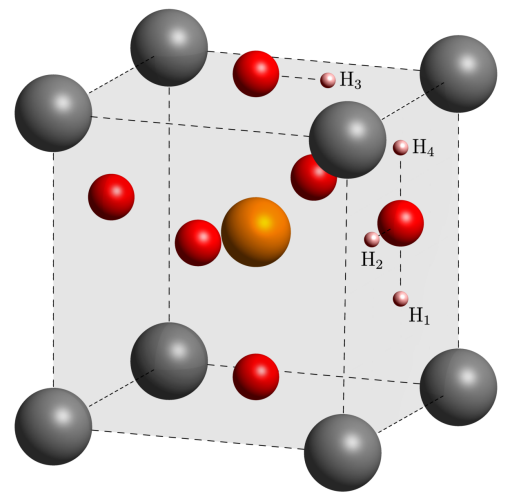

Figure 6.7: Possible locations for hydrogen occupancy as proposed by Alvine et al. [11]

occupancy locations in the PZT crystal structure. Thereafter, we will look at effects on the polarization and unit cell dimensions due to hydrogen occupying these interstitial locations. Finally we have identified possible escape paths available from each location, considering the nearest neighbour locations.

\subsubsection{Crystal structure of PZT}

As the first step of the process, we needed to determine a way to model the crystal structure of the PZT without any defects. Bulk of the previous work has been done using first principles DFT calculations [7,8,26]. Kitamura et al. [7] has developed an interatomic potential using a shell model for $\mathrm{PbZr}_{0.5} \mathrm{Ti}_{0.5} \mathrm{O}_{3}$ structure using results from first principles calculations. We decided to use first-principles calculations directly, since we need to accurately model the interactions of the hydrogen with the PZT structure. The potential of Kitamura et al. [7] has developed is based on DFT results which differs from experimental values for lattice constants and atom locations.

We used the VASP code which provides solutions to the Kohn-Sham functional (DFT) using pseudopotentials or the projector-augmented wave (PAW) method for calculations [43-45]. Local Density Approximation (LDA) [46], Generalized Gradient Approximation (GGA), generalized gradient approximation using the Perdew, Burke and Ernzerhof (PBE) simplification [47], and generalized gradient approximation for solids and surfaces (PBESol) [48] were available to estimate the exchange correlation energies. We used the lattice parameters and atomic coordinates shown in Table 6.1 and Table 6.2 to select the best functional from them. For this we selected a cell with 


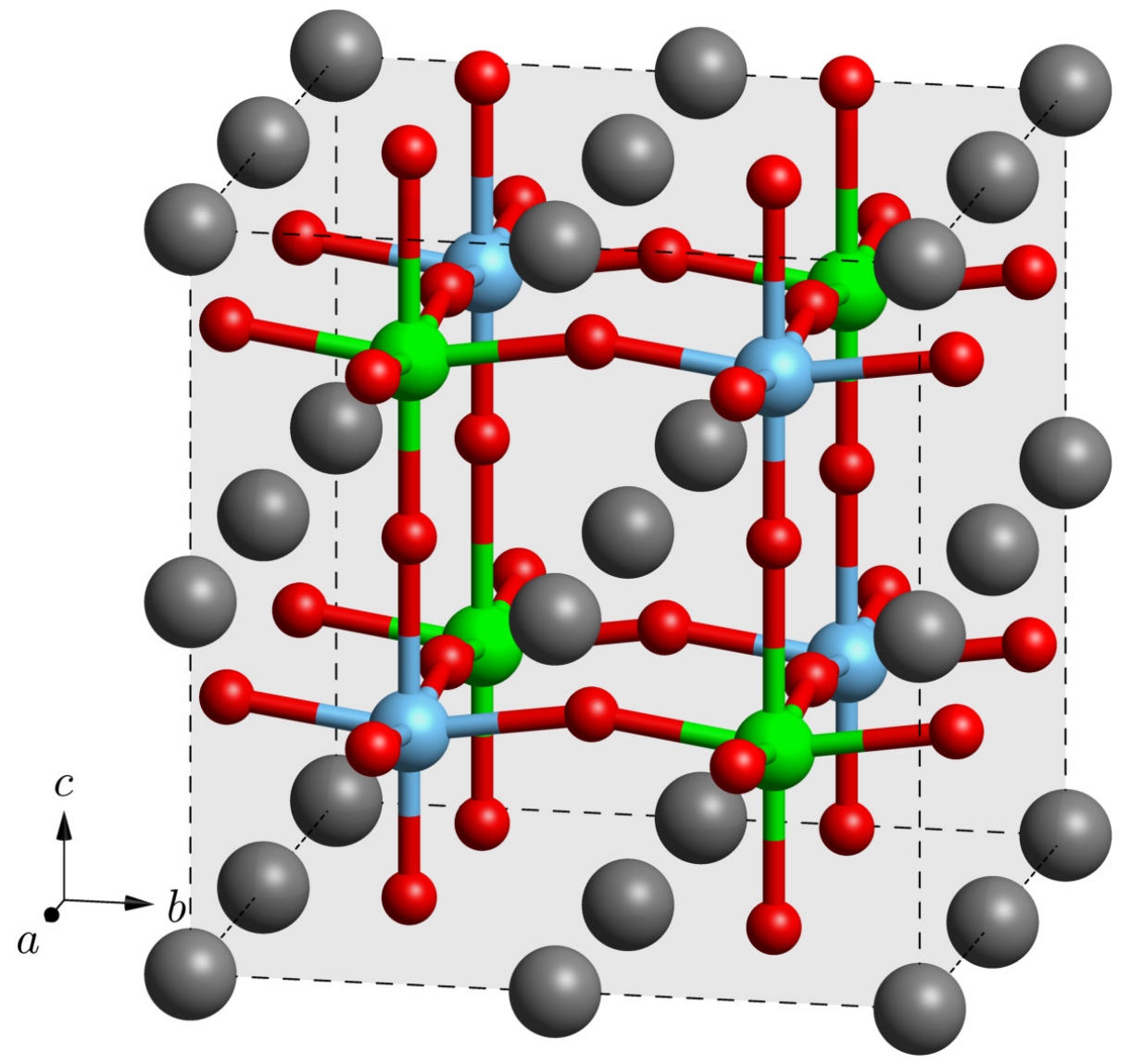

Figure 6.8: Atomic arrangement used to verify the model. While black colour spheres represent $\mathrm{Pb}$ atoms, $\mathrm{Zr}$ and $\mathrm{Ti}$ atoms are shown in blue and green colour spheres respectively. Red colour spheres are oxygen atoms

$2 \times 2 \times 2 \mathrm{ABO}_{3}$ (see Fig. 6.1(b)) cells with $\mathrm{Zr}$ and Ti occupying alternative sites as shown in Fig. 6.8. For these calculations we used periodic boundary conditions to represent a continuum. A K-point mesh density of $9 \times 9 \times 9$ and a plane wave energy cut off of $1000 \mathrm{eV}$ were used during these simulations to obtain accurate results. The shape and the dimensions of the cell was allowed to change during the simulations. Once a proper functional was selected, we did a sensitivity for the K-point mesh size and the plane wave cut off energy to select optimum values which gives good accuracy with better resource consumption. 


\subsubsection{Probable hydrogen occupancy locations}

As mentioned in Sec. 6.1.5 occupancy locations for hydrogen in the PZT lattice are not yet properly established. It has been found that hydrogen will mainly exist as a part of a hydroxyl group by forming a covalent bond with an oxygen atom [35]. Hence, we considered that hydrogen will only exist as a part of an $\mathrm{O}-\mathrm{H}$ group in the study. For each different oxygen atom shown in Fig. 6.8, we looked at possible locations with the $\mathrm{O}-\mathrm{H}$ bond oriented in multiples of $45^{\circ}$ angles in from the a, b, and c directions. 25 distinct arrangements were identified based on the symmetry of the structure. Using VASP code and PBESol functionals we allowed a set of systems systems, each with one hydrogen atom in the identified locations, to relax while allowing the volume and the shape of the cell to change unconstrained to simulate a stress-free condition. K-point mesh size of $5 \times 5 \times 5$ and a plane wave energy cut off of $600 \mathrm{eV}$ was used for these calculations. During this we assumed that the simulation cell is large enough so that interactions between two hydrogen atoms in two adjacent cells of periodic boundaries will not have any effect significant on the arrangement of an energy basin at an occupancy location. Once the stable occupancy sites were identified from DFT calculations, we analyzed them again in a larger cell with $4 \times 4 \times 4 \mathrm{ABO}_{3}$ unit cells to better identify the properties and effects of the occupancy sites better. For the larger model we found that a reduced mesh density of $3 \times 3 \times 3$ was more practical since the size of the reciprocal space would decrease, and that the simulations give pretty good accuracy even for the lowered mesh size.

\subsection{Results and Discussion}

\subsubsection{PZT crystal structure}

From the initial calculations, we obtained lattice parameters shown in Table 6.3 using LDA, GGA, PBE, and PBESol functionals. From these we can very well see that PBESol functionals are best suited for the DFT calculations. Lattice parameters obtained using PBESol functionals are very close to the experimental results shown in Table 6.1. Even compared to the previous studies based on DFT calculations, values from PBESol functionals show a much better agreement to the experimental values of the lattice parameters. For the average fractional positions of atoms in the unit cell, our results for the PBESol functional match well with the experimental 
Table 6.3: Lattice parameters for an $\mathrm{ABO}_{3}$ unit cell obtained from different functionals

\begin{tabular}{ccc}
\hline Functional & $a(\AA)$ & $c(\AA)$ \\
\hline \hline LDA & 3.988 & 4.122 \\
GGA & 4.043 & 4.329 \\
PBE & 4.042 & 4.355 \\
PBESol & 4.019 & 4.144 \\
\hline
\end{tabular}

Table 6.4: Average fractional coordinates in c-direction of an $\mathrm{ABO}_{3}$ unit cell from different functionals

\begin{tabular}{ccccc}
\hline Atom & LDA & GGA & PBE & PBESol \\
\hline \hline $\mathrm{Pb}$ & 0.000 & 0.000 & 0.000 & 0.000 \\
$\mathrm{Zr} / \mathrm{Ti}$ & 0.554 & 0.558 & 0.558 & 0.558 \\
$\mathrm{O}_{1}$ & 0.094 & 0.124 & 0.127 & 0.096 \\
$\mathrm{O}_{2}$ & 0.625 & 0.643 & 0.644 & 0.628 \\
\hline
\end{tabular}

work of Frantti et al. [23] compared to all other results. Hence, we used PBESol functional for our study. After a sensitivity study, we found that a K-point mesh size of $5 \times 5 \times 5$ and a plane wave energy cut off of $600 \mathrm{eV}$ would be sufficient for rest of the calculations with this simulation cell.

\subsubsection{Hydrogen occupancy locations}

Comparing 25 distinct possibilities for stable hydrogen occupancy locations, we identified that only five of them need to be considered in the rest of our work. The remaining arrangements either causes the PZT crystal to degenerate or relaxed into one of the five arrangements we identified. These results shows fair agreement with the predictions made in previous studies as shown in Fig. 6.7. Fig. 6.9 below shows the identified locations with respect to the analogous locations in Fig. 6.7. In all the locations, we can see that hydrogen has deviated closer to the Ti atoms from the symmetric positions. This makes sense, considering the smaller size and the lower 
Table 6.5: Total energy of the system with respect to the system with hydrogen occupying $\mathrm{H}_{1}$ location

\begin{tabular}{cc}
\hline Occupancy location & Relative Energy $(\mathrm{eV})$ \\
\hline \hline $\mathrm{H}_{2}$ & 0.398 \\
$\mathrm{H}_{3 T i}$ & 0.003 \\
$\mathrm{H}_{3 Z r}$ & -0.031 \\
$\mathrm{H}_{4}$ & 0.203 \\
\hline
\end{tabular}

atomic number (and hence the lower positive charge) of a Ti nucleus compared to a Zr nucleus.

Table 6.5 shows the total energy of the system for each of the occupancy location with respect to the system where hydrogen occupies the $\mathrm{H}_{1}$ location. The larger simulation cell mentioned in Sec. 6.2.2 was used for these simulations. From these results, we can see that the location $\mathrm{H} 3 \mathrm{Zr}$ has the minimum total energy value making it the most favourable site for hydrogen occupancy. The difference between sites $\mathrm{H}_{1}$, $\mathrm{H}_{3 T i}$, and $\mathrm{H}_{3 Z r}$ are relatively low, ranging only about $0.034 \mathrm{eV}$ between tham. The $\mathrm{H}_{2}$ location has the highest energy making it the least stable. The numbering of the occupancy locations in Fig. 6.7 is done in the order of increasing total energy of the $\mathrm{PbTiO}_{3}$ structure. From the current results we can see that this order is not the same in PZT.

When we looked at the charge density distribution in PZT without any defects (interstitial hydrogen), we can see a clear separation of the electronic clouds (see Fig. 6.10). We can see a clear seperation of the electronic clouds, which are mainly concentrated about oxygen atoms. This behaviour is similar to that of ionic crystals. This explains the high resistance mentioned in the previous studies.

For the models with interstitial hydrogen (Fig. 6.11 shows the charge density distribution for $\mathrm{H}_{1}$ location, where the other locations show similar charge distributions), we can clearly see that the electronic cloud is shared between hydrogen and the nearest oxygen atom showing characteristics of a covalent bond. From these we can clearly conclude that hydrogen is existing as a part of a hydroxy group (O-H) in 


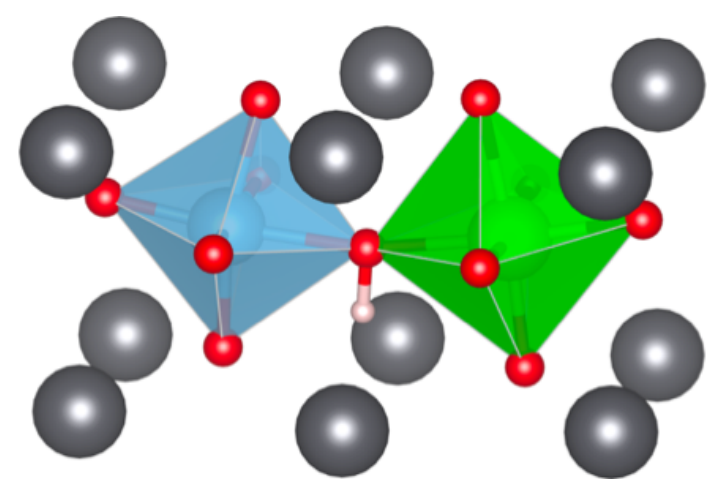

(a) For location $\mathrm{H}_{1}$ in Fig. $6.7\left(\mathrm{H}_{1}\right)$

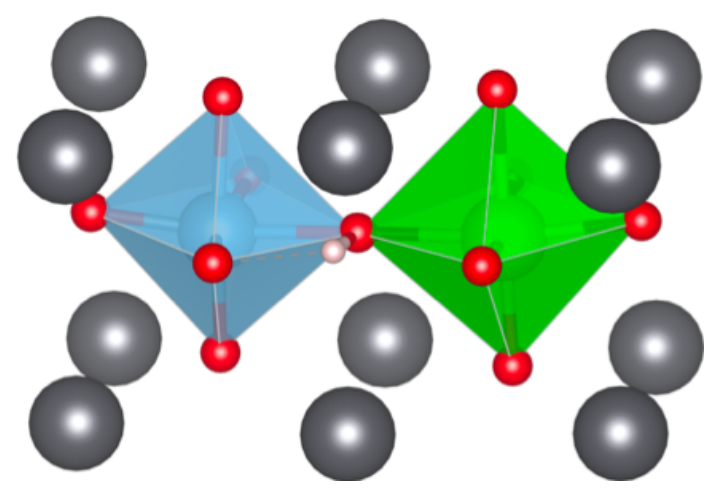

(b) For location $\mathrm{H}_{2}$ in Fig. $6.7\left(\mathrm{H}_{2}\right)$

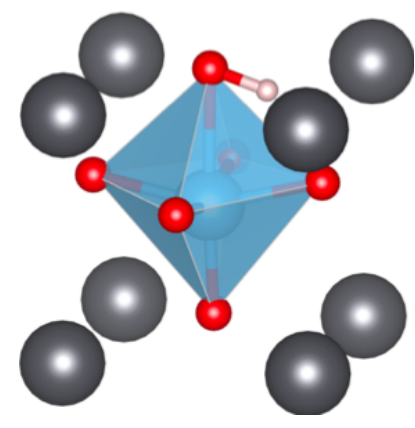

(c) For location $\mathrm{H}_{3}$ in Fig. 6.7 with B is $\mathrm{Zr}\left(\mathrm{H}_{3 Z r}\right)$

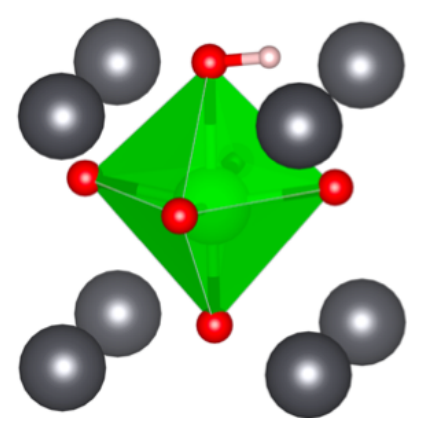

(d) For location $\mathrm{H}_{3}$ in Fig. 6.7 with $\mathrm{B}$ is $\mathrm{Ti}\left(\mathrm{H}_{3 T i}\right)$

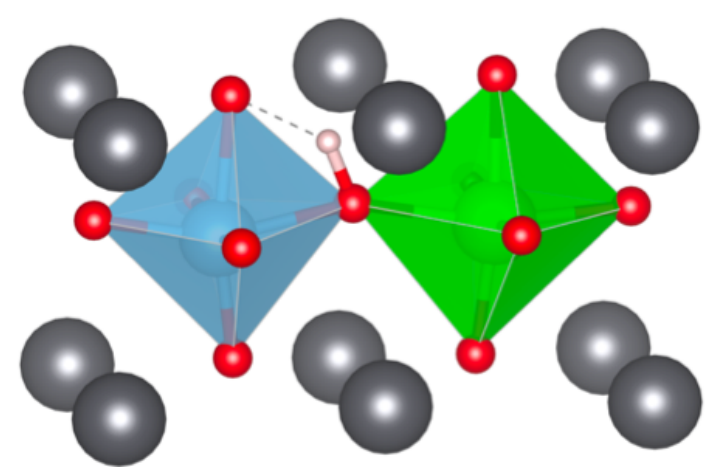

(e) For location $\mathrm{H}_{4}$ in Fig. $6.7\left(\mathrm{H}_{4}\right)$

Figure 6.9: Identified occupancy locations for hydrogen in the PZT lattice with respect to the analogous locations in Fig. 6.7. Colors for $\mathrm{Pb}, \mathrm{Zr}$, Ti and $\mathrm{O}$ atoms are the same as in Fig. 6.8, and the light pink sphere represent $\mathrm{H}$ atoms. The oxygen cage around $\mathrm{Ti}$ and $\mathrm{Zr}$ atoms are shown with octahedrons of the respective color 


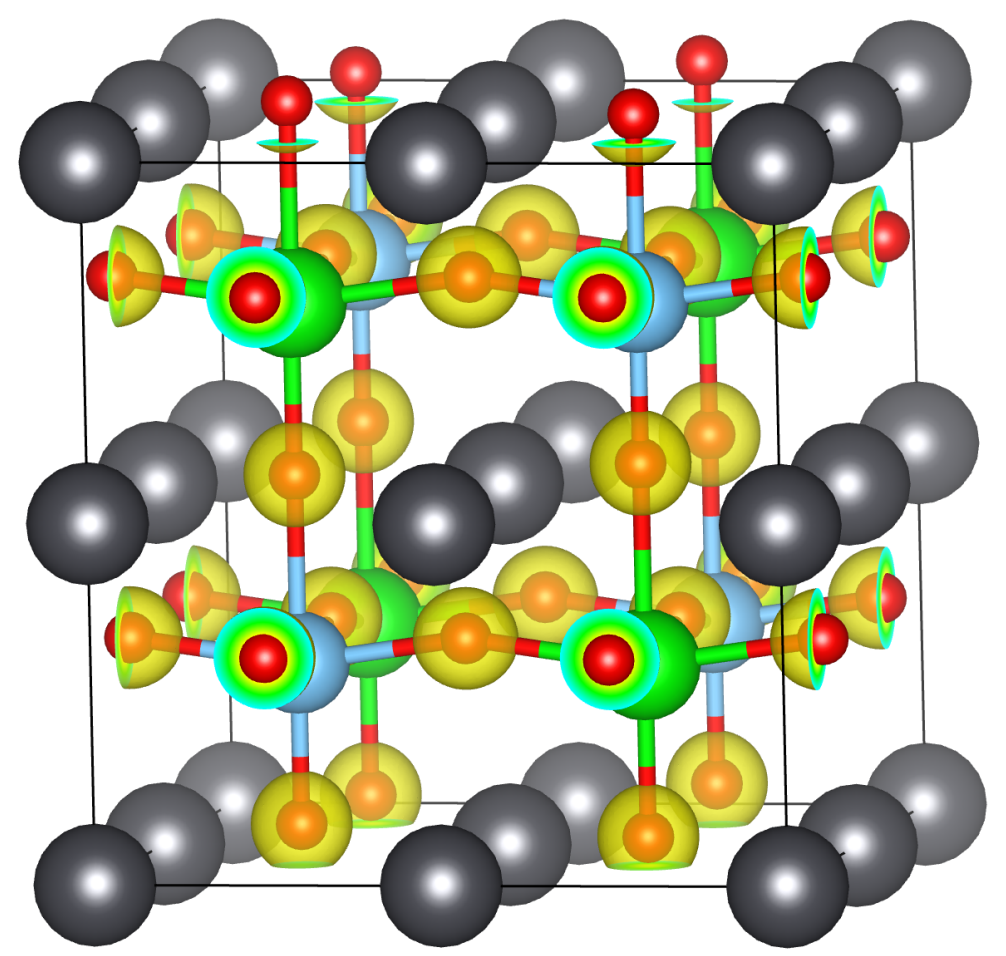

Figure 6.10: Charge density distribution for tetragonal PZT without any interstitial hydrogen. The colours of the atoms are the same as shown in Fig. 6.8. The yellow colour spheres enveloping the oxygen atoms shows that the electrons are concentrated around oxygen atoms 


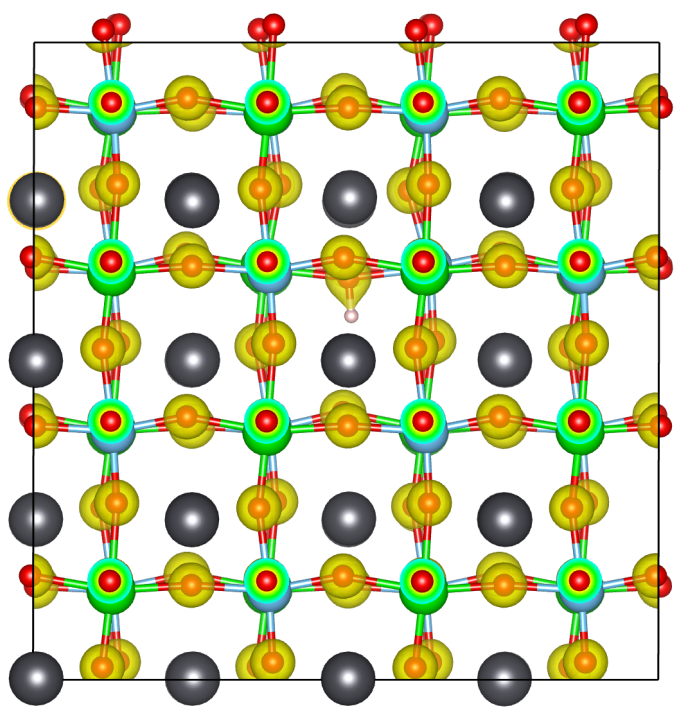

(a) Distribution of the electron clouds in the cell

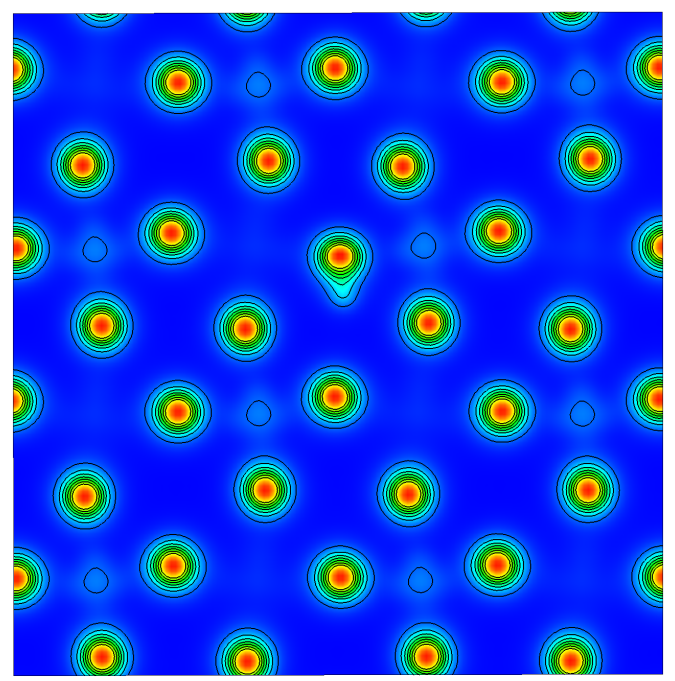

(b) Charge density plot on the plane with the hydrogen atom

Figure 6.11: Charge density of PZT with an interstitial hydrogen atom occupying $\mathrm{H}_{1}$ location

the lattice in the observed locations.

We looked at the effects of a hydrogen atom occupying each of these locations on the cell dimensions and the polarization (see Table 6.6). The polarization $(P$, dipole moment per unit volume) about the center of the simulation cell was calculated as,

$$
P=\frac{1}{V} \int_{V} \rho(\mathbf{r})\left(\mathbf{r}-\mathbf{r}_{\mathbf{c}}\right) d V
$$

where $\rho(\mathbf{r})$ is the charge density at position $\mathbf{r}, V$ is the total volume of the cell, and $\mathbf{r}_{\mathbf{c}}$ is the center of the simulation cell. Spontaneous polarization is defined as the difference in the polarization between the considered structure to the symmetric structure (cubic PZT for this case). Hence, the polarization value calculated will be equal to the spontaneous polarization, since the polarization about the center of the cell is zero for cubic PZT. From the values shown in Table 6.6, we can see that hydrogen does not cause any significant changes to the shape and the volume of the PZT cell. The highest change to the lattice parameter a and c seems to be when hydrogen occupies location $\mathrm{H} 1$, which are still less than $1 \%$. The minimum polarization for the Pure PZT in a single domain crystal is found to be $75 \mu \mathrm{C} / \mathrm{cm}^{2}$ [49], which agrees with the value we have obtained for the tetragonal PZT structure. With the presence 
Table 6.6: Lattice parameters, volume, and the polarization of PZT structures with and without hydrogen occupying the locations established to be stable occupancy sites

\begin{tabular}{ccccc}
\hline System & a $(\AA)$ & c $(\AA)$ & Volume $\left(\AA^{3}\right)$ & Polarization $\left(\mu \mathrm{C} / \mathrm{cm}^{2}\right)$ \\
\hline \hline Pure PZT & 4.018 & 4.141 & 66.86 & 85.58 \\
With $\mathrm{H}$ at $\mathrm{H}_{1}$ & 4.032 & 4.108 & 66.78 & 21.69 \\
With $\mathrm{H}$ at $\mathrm{H}_{2}$ & 4.024 & 4.131 & 66.95 & 24.46 \\
With H at $\mathrm{H}_{3 T i}$ & 4.024 & 4.136 & 66.96 & 25.49 \\
With H at $\mathrm{H}_{3 Z r}$ & 4.024 & 4.135 & 66.96 & 24.69 \\
With $\mathrm{H}$ at $\mathrm{H}_{4}$ & 4.026 & 4.131 & 66.95 & 25.33 \\
\hline
\end{tabular}

of hydrogen polarization has decreased significantly, with an average reduction of approximately $70 \%$. However, the polarization generally has very little dependence on the occupancy location as seen from the values.

\subsubsection{Escape paths for hydrogen diffusion}

After identifying the probable occupancy locations, we looked at possible escape paths for hydrogen to move from an occupied site to an adjacent vacant site. In this we only considered transitions to its closest set of sites. Fig. 6.12 shows the identified escape paths for each of the occupancy locations.

While these are identified as probable paths, a thorough investigation is required to find whether they can be accepted as transition steps for hydrogen diffusion. In future work, we will do this through first-principles and nudged elastic band calculations.

\subsection{Conclusion and Future Directions}

We have used first principles DFT calculations to successfully model the crystal structure of $\mathrm{PbZr}_{0.5} \mathrm{Ti}_{0.5} \mathrm{O}_{3}$ and then used the verified model to identify the most probable locations in the structure for hydrogen to occupy. We identified five such locations, which agrees the with hypothesis made in some previous studies based on material with similar crystal structures. Looking at the charge density plots we were able to 


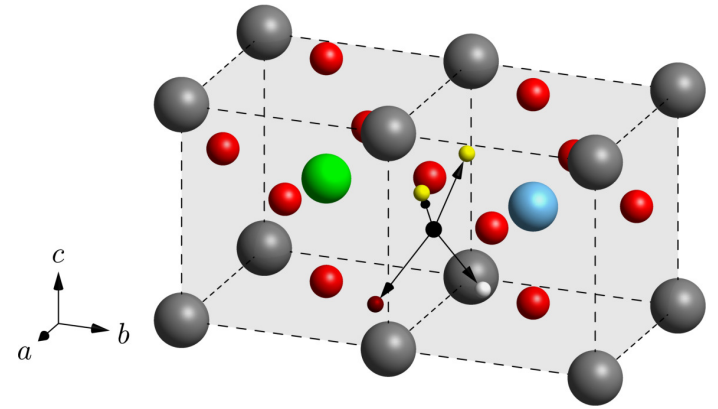

(a) $\mathrm{H}_{1}$

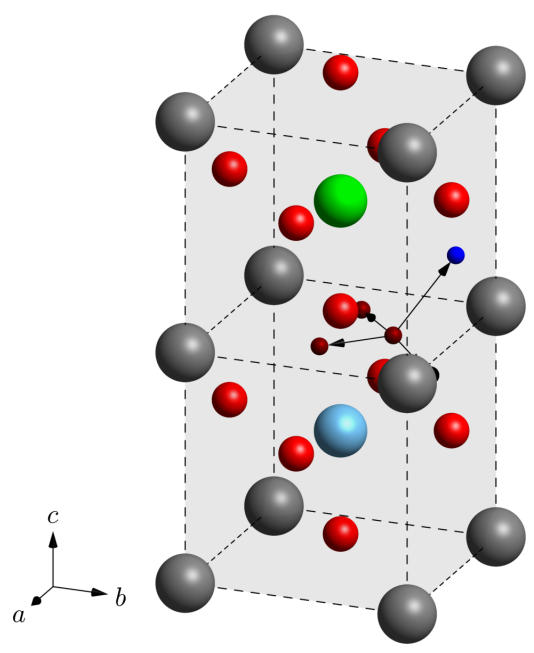

(c) $\mathrm{H}_{3 T i}$

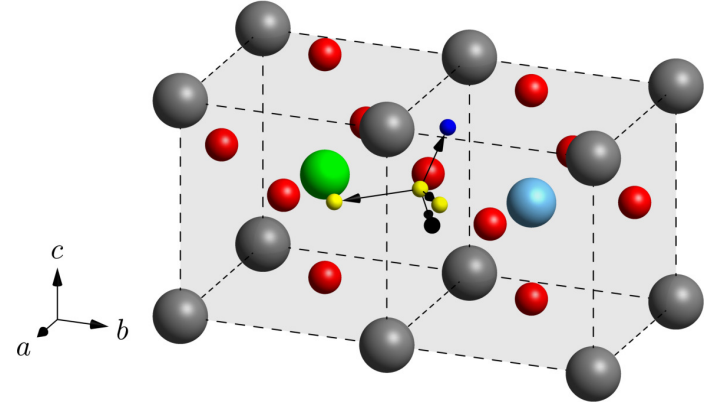

(b) $\mathrm{H}_{2}$

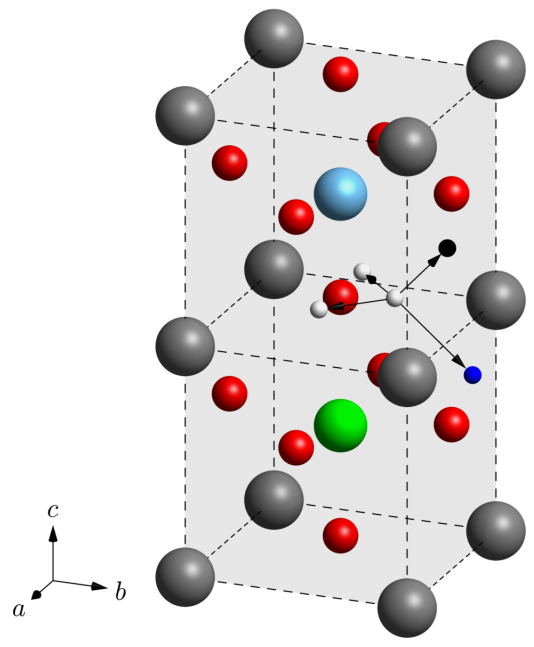

(d) $\mathrm{H}_{3 Z r}$

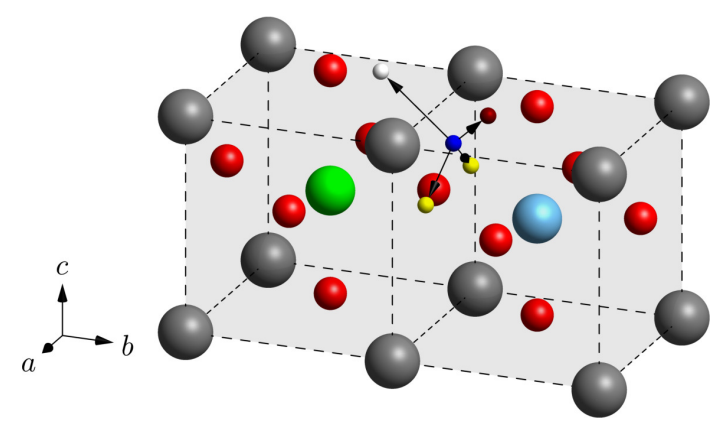

(e) $\mathrm{H}_{1}$

Figure 6.12: Proposed escape paths available at each occupancy location. $\mathrm{H}_{1}, \mathrm{H}_{2}$, $\mathrm{H}_{3 T i}, \mathrm{H}_{3 Z r}$, and $\mathrm{H}_{4}$ locations are represented by black, yellow, brown, white, and dark blue spheres respectively. The atoms in the structure are shown the same colored spheres as in Fig. 6.8 
identify that hydrogen indeed exists as a part of an O-H group. We studied the effects on dimensions, volume, and polarization in the presence of hydrogen using the results of our DFT calculations. While the effects on the dimensions and volume are negligible, it was found that hydrogen will cause the polarisation of the lattice to decrease considerably. Finally, we looked at the identified occupancy locations and proposed transition paths available for hydrogen to escape from these sites and diffuse in the PZT lattice.

Future direction is to analyze the identified transition steps and determine the diffusivity properties using DFT and nudged elastic band calculations. Once these properties are obtained, we will be able to use KMC simulations to predict the diffusion properties of hydrogen in the bulk PZT crystals.

\section{Bibliography}

[1] A. Gopejenko, S. Piskunov, and Y. F. Zhukovskii, "Ab initio modelling of the effects of varying $\mathrm{Zr}(\mathrm{Ti})$ concentrations on the atomic and electronic properties of stoichiometric PZT solid solutions," Comput. Theor. Chem., vol. 1104, pp. 5660, 2017.

[2] F. X. Li, R. K. N. D. Rajapakse, D. Mumford, and M. Gadala, "Quasi-static thermo-electro-mechanical behaviour of piezoelectric stack actuators," Smart Mater. Struct., vol. 17, no. 1, p. 015049, 2018.

[3] P. Cotterill, "The hydrogen embrittlement of metals," Prog. Mater. Sci., vol. 9, no. 4, pp. 205-250, 1961.

[4] H. C. Rogers, "Hydrogen embrittlement of metals," Science, vol. 10, no. 3819, pp. 1057-1064, 1968.

[5] M. Louthan Jr., G. Caskey Jr., J. Donovan, and D. Rawl Jr., "Hydrogen embrittlement of metals - fragilisation des métaux par l' hydrogéne wasserstoffversprödung in metallen," Mater. Sci. Eng., vol. 10, pp. 357-368, 1972.

[6] M. Liyanage, R. E. Miller, and R. K. N. D. Rajapakse, "Multi-scale approach for determining hydrogen diffusivity in zirconium," Model. Simul. Mater. Sci. Eng., vol. 26, p. 085002, 2018.

[7] T. Kitamura, Y. Umeno, F. Shang, T. Shimada, and K. Wakahara, "Development of Interatomic Potential for $\mathrm{Pb}(\mathrm{Zr}, \mathrm{Ti}) \mathrm{O}_{3}$ Based on Shell model," J. Solid Mech. Mater. Eng., vol. 1, no. 12, pp. 1423-1431, 2007.

[8] R. A. Ribeiro and S. R. de Lazaro, "DFT/PBE0 study on structural, electronic and dielectric properties of $\mathrm{SnZr}_{0.50} \mathrm{Ti}_{0.50} \mathrm{O}_{3}$ lead-free ferroelectric material," $J$. Alloy. Compd., vol. 714, pp. 553-559, 2017. 
[9] D.-J. Shin, S.-J. Jeong, C.-E. Seo, K.-H. Cho, and J.-H. Koh, "Multi-layered piezoelectric energy harvesters based on PZT ceramic actuators," Ceram. Int., vol. 41, pp. S686-S690, 2015. 9th Asian Meeting on Electroceramics (AMEC-9).

[10] Y. Singh, R. K. N. D. Rajapakse, E. Kjeang, and D. Mumford, "Performance of piezoelectric actuators in a hydrogen environment: Experimental study and finite element modelling," Int. J.Hydrogen Energ., vol. 40, no. 8, pp. 3370-3380, 2015 .

[11] K. J. Alvine, M. Vijayakumar, M. E. Bowden, A. L. Schemer-Kohrn, and S. G. Pitman, "Hydrogen diffusion in lead zirconate titanate and barium titanate," $J$. Appl. Phys., vol. 112, no. 4, p. 043511, 2012.

[12] K. J. Alvine, M. Vijayakumar, M. E. Bowden, A. L. Schemer-Kohrn, and S. G. Pitman, "Hydrogen diffusion in lead zirconate titanate and barium titanate," Journal of Applied Physics, vol. 112, no. 4, pp. 043511 1-6, 2012.

[13] F. P. Fengler, M. Pesic, S. Starschich, T. Schneller, U. Bottger, T. Schenk, M. H. Park, T. Mikolajick, and U. Schroeder, 2016 46th European Solid-State Device Research Conference (ESSDERC), pp. 369-372. Lausanne, Switzerland: IEEE, 2016.

[14] N. Jalili, J. Wagner, and M. Dadfarnia, "A piezoelectric driven ratchet actuator mechanism with application to automotive engine valves," Mechatronics, vol. 13, pp. 933-956, 2003.

[15] K. Kushida-Abdelghafar, H. Miki, K. Torii, and Y. Fujisaki, "Electrode-induced degradation of $\mathrm{Pb}\left(\mathrm{Zr}_{x} \mathrm{Ti}_{1-x}\right) \mathrm{O}_{3}$ (PZT) polarization hysteresis characteristics in Pt/PZT/Pt ferroelectric thin-film capacitors," Appl. Phys. Lett., vol. 69, no. 21, pp. 3188-3190, 1996.

[16] Y. Shimakawa and Y. Kubo, "Degradation of ferroelectric $\mathrm{Pb}(\mathrm{Zr}, \mathrm{Ti}) \mathrm{O}_{3}$ under reducing conditions," Appl. Phys. Lett., vol. 77, no. 16, pp. 2590-2592, 2000.

[17] Q. Wang and F. Li, "A low-working-field $(2 \mathrm{kV} / \mathrm{mm})$, large-strain $(>0.5 \%)$ piezoelectric multilayer actuator based on periodically orthogonal poled PZT ceramics," Sensor. Actuat. A: Phys., vol. 272, pp. 212-216, 2018.

[18] H. Zhao, Z.-R. Qu, H.-Y. Ye, and R.-G. Xiong, "In situ hydrothermal synthesis of tetrazole coordination polymers with interesting physical properties," Chem. Soc. Rev., vol. 37, no. 1, pp. 84-100, 2008.

[19] I. Grinberg, V. R. Cooper, and A. M. Rappe, "Oxide chemistry and local structure of $\mathrm{PbZr}_{x} \mathrm{Ti}_{1-x} \mathrm{O}_{3}$ studied by density-functional theory supercell calculations," Phys. Rev. B, vol. 69, no. 14, p. 144118, 2004.

[20] B. Noheda, D. E. Cox, G. Shirane, L. E. Cross, and S.-E. Park, "A monoclinic ferroelectric phase in the $\mathrm{Pb}\left(\mathrm{Zr}_{1-x} \mathrm{Ti}_{x}\right) \mathrm{O}_{3}$ solid solution," Appl. Phys. Lett., vol. 74, no. 14, pp. 2059-2061, 1999. 
[21] B. Noheda, J. A. Gonzalo, L. E. Cross, R. Guo, S.-E. Park, D. E. Cox, and G. Shirane, "Tetragonal-to-monoclinic phase transition in a ferroelectric perovskite: The structureof $\mathrm{PbZr}_{0.52} \mathrm{Ti}_{0.48} \mathrm{O}_{3}$," Phys. Rev. B, vol. 61, no. 13, pp. 8687-8695, 2000 .

[22] B. Jaffe, W. R. Cook, and H. L. Jaffe, Piezoelectric ceramics. London and New York: Academic Press, 1971.

[23] J. Frantti, S. Ivanov, S. Eriksson, H. Rundlöf, V. Lantto, J. Lappalainen, and M. Kakihana, "Phase transitions of $\mathrm{Pb}\left(\mathrm{Zr}_{x} \mathrm{Ti}_{1-x}\right) \mathrm{O}_{3}$ ceramics," Phys. Rev. B, vol. 66, no. 6, p. 064108, 2002.

[24] N. Zhang, H. Yokota, A. M. Glazer, and P. A. Thomas, "Neutron powder diffraction refinement of $\mathrm{PbZr}_{1-x} \mathrm{Ti}_{x} \mathrm{O}_{3}, "$ Acta Crystallogr. B, vol. 67, no. 5, pp. 386$398,2011$.

[25] C. L. Jia, J. R. Contreras, U. Poppe, H. Kohlstedt, R. Waser, and $\mathrm{K}$. Urban, "Lattice strain and lattice expansion of the $\mathrm{SrRuO}_{3}$ layers in $\mathrm{SrRuO}_{3} / \mathrm{PbZr}_{0.52} \mathrm{Ti}_{0.48} \mathrm{O}_{3} / \mathrm{SrRuO}_{3}$ multilayer thin films," J. Appl. Phys., vol. 92, no. 1, pp. 101-105, 2002.

[26] G. Sághi-szabó, R. E. Cohen, and H. Krakauer, "First-principles study of piezoelectricity in tetragonal $\mathrm{PbTiO}_{3}$ and $\mathrm{PbZr}_{1 / 2} \mathrm{Ti}_{1 / 2} \mathrm{O}_{3}$," Phys. Rev. B, vol. 59, no. 20, pp. 12771-12776, 1999.

[27] M. Wu, H. Huang, W. Chu, L. Guo, L. Qiao, J. Xu, and T.-Y. Zhang, "Tuning the Ferroelectric and Piezoelectric Properties of $0.91 \mathrm{~Pb}\left(\mathrm{Zn}_{1 / 3} \mathrm{Nb}_{2 / 3}\right) \mathrm{O}_{3}-0.09 \mathrm{PbTiO}_{3}$ Single Crystals and Lead Zirconate Titanate Ceramics by Doping Hydrogen," $J$. Phys. Chem. C, vol. 114, no. 21, pp. 9955-9960, 2010.

[28] K. Xiong and J. Robertson, "Hydrogen-induced defects and degradation in oxide ferroelectrics," Appl. Phys. Lett., vol. 85, no. 13, pp. 2577-2579, 2004.

[29] X.-H. Guo, S.-Q. Shi, and L.-J. Qiao, "Simulation of Hydrogen Diffusion and Initiation of Hydrogen-Induced Cracking in PZT Ferroelectric Ceramics Using a Phase Field Model," J. Am. Ceram. Soc., vol. 90, no. 9, pp. 2868-2872, 2007.

[30] Y. Fujisaki, K. Kushida-Abdelghafar, Y. Shimamoto, and H. Miki, "The effects of the catalytic nature of capacitor electrodes on the degradation of ferroelectric $\mathrm{Pb}(\mathrm{Zr}, \mathrm{Ti}) \mathrm{O}_{3}$ thin films during reductive ambient annealing," J. Appl. Phys., vol. 82, no. 1, pp. 341-344, 1997.

[31] J. P. Han and T. P. Ma, "Electrode dependence of hydrogen-induced degradation in ferroelectric $\mathrm{Pb}(\mathrm{Zr}, \mathrm{Ti}) \mathrm{O}_{3}$ and $\mathrm{SrBi}_{2} \mathrm{Ta}_{2} \mathrm{O}_{9}$ thin films," Appl. Phys. Lett., vol. 71, no. 9, pp. 1267-1269, 1997.

[32] J. K. Lee, Y. Park, and I. Chung, "Investigation of hydrogen-induced degradation in $\mathrm{Pb}\left(\mathrm{Zr}-x \mathrm{Ti}_{1-x}\right) \mathrm{O}_{3}$ thin film capacitors for the application of memory devices," J. Appl. Phys., vol. 92, no. 5, pp. 2724-2728, 2002.

[33] A. Shafiei, C. Oprea, A. Alfantazi, and T. Troczynski, "In situ monitoring of the effects of hydrogen on $\mathrm{Pb}(\mathrm{Zr}, \mathrm{Ti}) \mathrm{O}_{3}$ structure," J. Appl. Phys., vol. 109, no. 11, p. 114108, 2011. 
[34] X. Peng, Y. J. Su, K. W. Gao, L. J. Qiao, and W. Y. Chu, "Hydrogen fissure in PZT ferroelectric ceramic," Materials Letters, vol. 58, no. 15, pp. 2073-2075, 2004.

[35] S. Aggarwal, S. R. Perusse, C. W. Tipton, R. Ramesh, H. D. Drew, T. Venkatesan, D. B. Romero, V. B. Podobedov, and A. Weber, "Effect of hydrogen on $\mathrm{Pb}(\mathrm{Zr}, \mathrm{Ti}) \mathrm{O}_{3}$-based ferroelectric capacitors," Appl. Phys. Lett., vol. 73, no. 14, pp. 1973-1975, 1998.

[36] N. R. Rajopadhye, S. V. Bhoraskar, S. Badrinarayan, and S. A. P. B., "Photoacoustic and X-ray photoelectron spectroscopic studies in reduced lead zirconate titanate ceramics," J. Mater. Sc., vol. 23, no. 7, pp. 2631-2636, 1988.

[37] J.-M. Koo, T. Kim, and J. Kim, "Hydrogen induced degradation ]phenomena of pzt ferroelectric capacitors," in Proc. 2000 12th IEEE Int. Symp. Appl. Ferr. (S. K. Streiffer, B. J. Gibbons, T. Tsurumi, and M. A. A. E. Zurbuchen, eds.), vol. 2, (Honolulu, HI, USA), pp. 591-594, Institute of Electrical and Electronics Engineers, Inc., 2000.

[38] H. Y. Huang, W. Y. Chu, Y. J. Su, K. W. Gao, J. X. Li, and L. J. Qiao, "Hydrogen-induced semiconductor transformation of lead zirconate titanate ferroelectric ceramics," J. Am. Ceram. Society, vol. 90, no. 7, pp. 2062-2066, 2007.

[39] Y. Wang, W. Y. Chu, L. J. Qiao, and Y. J. Su, "Hydrogen-induced delayed fracture of PZT ceramics during dynamic charging under constant load," Materials Science and Engineering, vol. 98, no. 1, pp. 1-5, 2003.

[40] W. Chen, L. Li, Y. Wang, and Z. Gui, "Effects of electrochemical hydrogen charging on lead-based relaxor ferroelectric multilayer ceramic capacitors," $J$. Mater. Res., vol. 13, no. 05, pp. 1110-1112, 1998.

[41] D. Houde, Y. Lépine, C. Pépin, S. Jandl, and J. L. Brebner, "High-resolution infrared spectroscopy of hydrogen impurities in strontium titanate," Phys. Rev. $B$, vol. 35, no. 10, pp. 4948-4953, 1987.

[42] C. H. Park and D. J. Chadi, "Effect of interstitial hydrogen impurities on ferroelectric polarization in $\mathrm{PbTiO}_{3}$," Phys. Rev. Lett., vol. 84, no. 20, pp. 4717-4720, 2000 .

[43] G. Kresse and J. Hafner, "Ab initio molecular dynamics for liquid metals," Physical Review B, vol. 47, no. 1, pp. 558-561, 1993.

[44] G. Kresse and J. Furthmüller, "Efficiency of ab-initio total energy calculations for metals and semiconductors using a plane-wave basis set," Computational Materials Science, vol. 6, no. 1, pp. 15-50, 1996.

[45] G. Kresse and J. Furthmüller, "Efficient iterative schemes for $<\mathrm{i}>$ ab initio $</ \mathrm{i}>$ total-energy calculations using a plane-wave basis set," Physical Review B, vol. 54, no. 16, pp. 11169-11186, 1996.

[46] W. Kohn and L. J. Sham, "Self-consisten equations includingexchange and correlation effects," Physical Review, vol. 140, p. A1133-A1138, 1965. 
[47] J. P. Perdew, K. Burke, and M. Ernzerhof, "Generalized Gradient Approximation Made Simple," Physical Review Letters, vol. 77, no. 18, pp. 3865-3868, 1996.

[48] J. P. Perdew, A. Ruzsinszky, G. I. Csonka, O. A. Vydrov, G. E. Scuseria, L. A. Constantin, X. Zhou, and K. Burke, "Restoring the Density-Gradient Expansion for Exchange in Solids and Surfaces," Phys. Rev. Lett., vol. 100, no. 13, p. 136406 , 2008.

[49] D. Berlincourt and H. H. A. Krueger, "Domain processes in lead titanate zirconate and barium titanate ceramics," J. Appl. Phys., vol. 30, no. 11, pp. 1840$1810,1959$. 


\section{Chapter 7}

\section{Conclusion and Future work}

\subsection{Summary and Conclusions}

Hydrogen diffusion in zirconium is an important research topic since, Zr is a popular material in the nuclear industry and $\mathrm{H}$ causes a multitude of problems (through formation of hydrides). Hence, monitoring of the hydride content in $\mathrm{Zr}$ pressure tubes, which are used as cladding for nuclear fuel, is of utmost importance. Denuded zones found close to the surface of these pressure tubes deter this monitoring process. One of the hypotheses put forth as the reason for the formation of denuded zones is that high $\mathrm{O}$ concentration close to the surface of the pressure tubes cause the $\mathrm{H}$ to slow down, making it difficult for $\mathrm{H}$ atoms to cluster and make large hydrides. The current study started with the intent of checking the validity of the hypothesis by quantifying the effect $\mathrm{O}$ has on the diffusion of $\mathrm{H}$ in $\mathrm{Zr}$, with the use of computer based simulations.

As the starting point of the study we developed a multi-scale model with the use of first-principles calculations and KMC simulations to model $\mathrm{H}$ diffusion in systems where the occupancy locations and the transition paths available for diffusion are properly recognized. We used the results available from previous studies for $\mathrm{H}$ diffusion in $\mathrm{Zr}$, both through experimental and computational means, to validate the model we use. With the simulations, we were able to ascertain the diffusivity for $\mathrm{H}$ in Zr. Further investigation of the diffusion process allowed us to investigate the anisotropic nature of $\mathrm{H}$ diffusion, where we found that the diffusion is nearly isotropic in the anisotropic Zr lattice. By looking at individual diffusion steps we identified the reasons for this behavior.

In the next study we were able to improve the accuracy and the speed of the 
diffusion model by introducing the effects of ZPE and quantum tunnelling to the hopping rates, and improving its efficiency. Thereafter we looked at how the stability of $\mathrm{H}$ occupancy locations in the Zr lattice and the transition steps available for moving between these occupancy locations are affected by neighboring $\mathrm{O}$ atoms. Through this we found that $\mathrm{O}$ atoms causes some of the occupancy locations to increase in stability and make it difficult for $\mathrm{H}$ atoms to leave these sites. When the number of $\mathrm{O}$ atoms in the vicinity increases, the effect is also increased. Using the diffusion model we were able to calculate the effect $\mathrm{O}$ has on $\mathrm{H}$ diffusivity in $\mathrm{Zr}$. Here we found that $\mathrm{O}$ does indeed decrease the diffusivity of $\mathrm{H}$ in $\mathrm{Zr}$. When we looked at the reasons for the decrease in diffusivity, we found that in addition to the decrease in hopping rates due to the increased stability, $\mathrm{H}$ traps formed by a cluster of nearest neighbor interstitial sites further decreases the $\mathrm{H}$ diffusivity when $\mathrm{O}$ concentration increases.

While it is proven that $\mathrm{H}$ diffusivity is decreased by $\mathrm{O}$, we used a $\mathrm{O}$ concentration profile to see whether this is the cause for the formation of denuded zones. As the starting point we looked at the change of the size of the precipitates under the influence of $\mathrm{O}$. Then we predicted the $\mathrm{O}$ concentration profile closer to the surface of the pressure tubes by simulating the service conditions. Looking at the change in precipitate size and the $\mathrm{O}$ concentration profile we see that the $\mathrm{O}$ concentration found through the depth of denuded zone is not sufficient to change the size of the hydrides to a noticeable level. This help us to reject the hypothesis proposed here as the cause for the formation of denuded zones.

Looking at other hypothesis available for the formation of denuded zones, we saw that the stress levels close to the oxide metal interface is quite significant. Most studies looking at the effects of stress only considers the effect of stress gradient as a drift force in the modified diffusion flux equation. However, it is understood that at high stress levels, diffusivity can change as a function of stress. Hence, we wanted to look at how diffusivity changes with hydrostatic stress applied to the Zr lattice. Using the developed diffusion model we investigated the variation of diffusivity for a broad stress range including tensile and compressive stresses. From this we found that compressive stresses decrease the diffusivity of $\mathrm{H}$ while tensile stresses increase the diffusivity. We quantified the change for the operating temperature range in $\mathrm{Zr}$ pressure tubes.

While this thesis looks at different phenomena related to $\mathrm{H}$ diffusion in $\mathrm{Zr}$, we wanted to extend this to another material which undergoes $\mathrm{H}$ based degradation. We 
found that PZT, a very popular piezoelectric material, also undergoes degradation when exposed to $\mathrm{H}$, through loss of piezoelectric and strength properties. While $\mathrm{H}$ degradation is a major issue in PZT, there are very few studies which investigate the $\mathrm{H}$ diffusion behavior, and the results from them are inconclusive. We took the first steps in filling this void by carrying out first-principles calculations to identify the occupancy locations for $\mathrm{H}$ in the PZT lattice, considering a variety of possible locations. We found five distinct locations where $\mathrm{H}$ can be stably occupied. While we didn't have any previous results to validate this for PZT, we found that for $\mathrm{PbTiO}_{3}$ similar locations are occupied by $\mathrm{H} . \mathrm{PbTiO}_{3}$ has a very similar crystal structure and is a constituent material of PZT. We found that each of these locations decrease the polarisation of the lattice, giving evidence to the fact that $\mathrm{H}$ affects the piezoelectric properties of PZT. After identifying the occupancy locations we looked at the nearest neighbour sites to propose transition steps available for hydrogen to diffuse through PZT.

\subsection{Future work}

Through the thesis we found that the hypothesis suggesting $\mathrm{O}$ slows down $\mathrm{H}$ diffusion near the pressure tube surfaces does not explain the formation of denuded zones. Further investigation is required to understand the reason for the formation of the denuded zones. Once the reason is found, a quantified analysis is required to predict the (maximum) thickness of the denuded zones which can occur in pressure tubes to modify the scrape sampling method.

While we carried out a study and identified the effect hydrostatic stress has on $\mathrm{H}$ diffusion in $\mathrm{Zr}$, we need to carry out a study to look at the effect this will have on diffusion more generally. Understanding the stress levels for which the change in diffusivity becomes significant is one idea which comes to mind.

We have identified the occupancy locations and proposed transition paths available for $\mathrm{H}$ to diffuse through PZT lattices. Next, it is required to carry out further studies to find the hopping rates for each of these transitions which are to be used as input to KMC simulations. Then we can ultimately predict the diffusion rates of $\mathrm{H}$ in PZT. 


\section{List of References}

[1] Capital Energy, "Hydriding." http://capitalenergy.biz/?p=25905. Accessed: 2017 Jul. 04.

[2] J. Hsu, J. Cui, A. Celovsky, E. Tulk, and Kinetrics, "Prediction of hydrogen isotope denuded zone in pressure tube," 2016. Private presentation.

[3] S. Aggarwal, S. R. Perusse, C. W. Tipton, R. Ramesh, H. D. Drew, T. Venkatesan, D. B. Romero, V. B. Podobedov, and A. Weber, "Effect of hydrogen on $\mathrm{Pb}(\mathrm{Zr}, \mathrm{Ti}) \mathrm{O}_{3}$-based ferroelectric capacitors," Appl. Phys. Lett., vol. 73, no. 14, pp. 1973-1975, 1998.

[4] K. J. Alvine, M. Vijayakumar, M. E. Bowden, A. L. Schemer-Kohrn, and S. G. Pitman, "Hydrogen diffusion in lead zirconate titanate and barium titanate," J. Appl. Phys., vol. 112, no. 4, p. 043511, 2012.

[5] World Nuclear Association, "Nuclear Power in the World Today." https://www.world-nuclear.org/information-library/ current-and-future-generation/nuclear-power-in-the-world-today . aspx. Accessed: 2020 Feb. 29.

[6] U.S. Energy Information Administration, "Nuclear explained - Nuclear power plants." https://www.eia.gov/energyexplained/nuclear/ nuclear-power-plants. php. Accessed: 2020 Feb. 29.

[7] S. Schlömer, T. Bruckner, L. Fulton, E. Hertwich, A. McKinnon, D. Perczyk, J. Roy, R. Schaeffer, R. S. amd P. Smith, and R. Wiser, "Annex iii: Technologyspecific cost and performance parameters.", in Climate Change 2014: Mitigation of Climate Change. Contribution of Working Group III to the Fifth Assessment Report of the Intergovernmental Panel on Climate Chang (O. Edenhofer, R. Pichs-Madruga, Y. Sokona, E. Farahani, S. Kadner, K. Seyboth, A. Adler, I. Baum, S. Brunner, P. Eickemeier, B. Kriemann, J. Savolainen, S. Schlömer, C. von Stechow, T. Zwickel, and J. C. Minx, eds.), p. 1329-1356, Cambridge, United Kingdom and New York, NY, USA: Cambridge University Press, 2014.

[8] A. Markandya and P. Wilkinson, "Electricity generation and health," Lancet, vol. 370, pp. 979-990, 2007.

[9] Natural Resources Canada, "Uranium and nuclear power facts." https:// www.nrcan.gc.ca/science-data/data-analysis/energy-data-analysis/ energy-facts/uranium-and-nuclear-power-facts/20070\#L1. Accessed: 2020 Feb. 29. 
[10] J. Parson, "Nasa plans to put nuclear reactors on mars to power a human colony - mirror online." https://www.mirror.co.uk/science/ nasa-plans-put-nuclear-reactors-10729812. Accessed: 2020 Feb. 17.

[11] E. Chajduk and A. Bojanowska-Czajka, "Corrosion mitigation in coolant systems in nuclear power plants," Progress in Nuclear Energy, vol. 88, pp. 1-9, 2016.

[12] A. T. Motta, A. Couet, and R. J. Comstock, "Corrosion of zirconium alloys used for nuclear fuel cladding," Annual Review of Materials Research, vol. 45, p. 311-343, 2015.

[13] International Atomic Energy Agency, Corrosion of zirconium alloys in nuclear power plants. Vienna, Austria: International Atomic Energy Agency, 1993.

[14] M. S. Blackmur, J. D. Robson, M. Preuss, O. Zanellato, R. J. Cernik, S. Q. Shi, F. Ribeiro, and J. Andrieux, "Zirconium hydride precipitation kinetics in zircaloy-4 observed with synchrotron x-ray diffraction," Journal of Nuclear Materials, vol. 464, p. 160-169, 2015.

[15] R. Tapping, "Corrosion issues in pressurized heavy water reactor (PHWR/CANDUR) systems," in Nuclear Corrosion Science and Engineering (D. Féron, ed.), Woodhead Publishing Series in Energy, pp. 581 - 633, Woodhead Publishing, 2012.

[16] R. Krishnan and M. K. Asundi, "Zirconium alloys in nuclear technology," in Proceedings of the Indian Academy of Sciences Section C: Engineering Sciences, vol. 4, pp. 41-56, 1981.

[17] M. V. Glazoff, "Modeling of some physical properties of zirconium alloys for nuclear applications in support of ufd campaign," tech. rep., Idaho National Laboratory, UFD Campaign, Idaho Falls, Idaho, Aug 2013.

[18] S. Suman, M. K. Khan, M. Pathak, R. N. Singh, and J. K. Chakravartty, "Hydrogen in Zircaloy: Mechanism and its impacts," International Journal of Hydrogen Energy, vol. 40, pp. 5976-5994, 2015.

[19] D. Hardie, "The effect of oxygen on the precipitation of hydrogen from zirconium," Journal of Nuclear Materials, vol. 17, pp. 88-90, 1965.

[20] E. Polatidis, P. Frankel, J. Wei, M. Klaus, R. Comstock, A. Ambard, S. Lyon, R. Cottis, and M. Preuss, "Residual stresses and tetragonal phase fraction characterisation of corrosion tested zircaloy-4 using energy dispersive synchrotron x-ray diffraction," Journal of Nuclear Materials, vol. 432, no. 1, pp. 102 - 112, 2013.

[21] P. Platt, E. Polatidis, P. Frankel, M. Klaus, M. Gass, R. Howells, and M. Preuss, "A study into stress relaxation in oxides formed on zirconium alloys," Journal of Nuclear Materials, vol. 456, pp. 415 - 425, 2015.

[22] Y.-S. Kim, Y.-H. Jeong, and J.-N. Jang, "Stress measurements during thin film zirconium oxide growth," Journal of Nuclear Materials, vol. 412, pp. 217-220, 2011. 
[23] R. A. Ribeiro and S. R. de Lazaro, "DFT/PBE0 study on structural, electronic and dielectric properties of $\mathrm{SnZr}_{0.50} \mathrm{Ti}_{0.50} \mathrm{O}_{3}$ lead-free ferroelectric material," $J$. Alloy. Compd., vol. 714, pp. 553-559, 2017.

[24] T. Kitamura, Y. Umeno, F. Shang, T. Shimada, and K. Wakahara, "Development of Interatomic Potential for $\mathrm{Pb}(\mathrm{Zr}, \mathrm{Ti}) \mathrm{O}_{3}$ Based on Shell model," J. Solid Mech. Mater. Eng., vol. 1, no. 12, pp. 1423-1431, 2007.

[25] D.-J. Shin, S.-J. Jeong, C.-E. Seo, K.-H. Cho, and J.-H. Koh, "Multi-layered piezoelectric energy harvesters based on PZT ceramic actuators," Ceram. Int., vol. 41, pp. S686-S690, 2015. 9th Asian Meeting on Electroceramics (AMEC-9).

[26] Y. Singh, R. K. N. D. Rajapakse, E. Kjeang, and D. Mumford, "Performance of piezoelectric actuators in a hydrogen environment: Experimental study and finite element modelling," Int. J.Hydrogen Energ., vol. 40, no. 8, pp. 3370-3380, 2015.

[27] F. Willaime, "Ab initio study of self-interstitials in hcp-Zr," Journal of Nuclear Materials, vol. 323, pp. 205-212, 2003.

[28] J. L. Zarestky, Lattice dynamics of hcp and bcc zirconium. PhD thesis, Iowa State University, 1979.

[29] N. A. P. K. Kumar, Hydride formation in zirconium alloys. PhD thesis, McGill University, 2011.

[30] C. Anghel, Modified oxygen and hydrogen transport in Zr-based oxides. PhD thesis, Royal Institute of Technology, KTH, 2006.

[31] J. Song and W. A. Curtin, "A nanoscale mechanism of hydrogen embrittlement in metals," Acta Materialia, vol. 59, p. 1557-1569, 2011.

[32] M. L. Martin, B. P. Somerday, R. O. Ritchie, P. Sofronis, and I. M. Robertson, "Hydrogen-induced intergranular failure in nickel revisited," Acta Materialia, vol. 60, p. 2739-2745, 2012.

[33] A. Turnbull, "Perspectives on hydrogen uptake, diffusion and trapping," International Journal of Hydrogen Energy, vol. 40, p. 16961-16970, 2015.

[34] G. Lu and E. Kaxiras, "Hydrogen embrittlement of aluminum: The crucial role of vacancies," Physical Review Letters, vol. 94, no. 15, pp. 155501 1-5, 2005.

[35] J. Song and W. A. Curtin, "Atomic mechanism and prediction of hydrogen embrittlement in iron.," Nature materials, vol. 12, no. 2, pp. 145-151, 2013.

[36] M. Christensen, W. Wolf, C. Freeman, E. Wimmer, R. B. Adamson, L. Hallstadius, P. Cantonwine, and E. V. Mader, "Effect of hydrogen on dimensional changes of zirconium and the influence of alloying elements: First-principles and classical simulations of point defects, dislocation loops, and hydrides," in STP1543-EB Zirconium in the Nuclear Industry (B. Comstock and P. Barberis, eds.), vol. 17, pp. 55-92, West Conshohocken, PA: ASTM International, 2015. 
[37] M. Ma, W. Xiang, B. Tang, L. Liang, L. Wang, and X. Tan, "Non-isothermal and isothermal hydrogen desorption kinetics of zirconium hydride," Journal of Nuclear Materials, vol. 467, pp. 349-356, 2015.

[38] A. E. Evsin, L. B. Begrambekov, A. I. Gumarov, A. S. Kaplevsky, A. G. Luchkin, L. R. Tagirov, and I. R. Vakhitov, "Trapping and desorption of hydrogen isotopes under irradiation of zirconium by deuterium atoms of thermal energies," Vacuum, vol. 129, p. 183-187, 2016.

[39] P. A. Burr, S. T. Murphy, S. C. Lumley, M. R. Wenman, and R. W. Grimes, "Hydrogen accommodation in zr second phase particles: implications for $\mathrm{h}$ pick-up and hydriding of zircaloy-2 and zircaloy-4," Corrosion Science, vol. 69, pp. 1-4, 2013.

[40] S. C. Lumley, R. W. Grimes, S. T. Murphy, P. A. Burr, A. Chroneos, P. R. Chard-Tuckey, and M. R. Wenman, "The thermodynamics of hydride precipitation: The importance of entropy, enthalpy and disorder," Acta Materialia, vol. 79, pp. 351-362, 2014.

[41] J. J. Kearns, "Terminal solubility and partitioning of hydrogen in the alpha phase of zirconium, Zircaloy-2 and Zircaloy-4," Journal of Nucelar Materials, vol. 22, p. 292-303, 1967.

[42] G. F. Slattery, "The terminal solubility of hydrogen in zirconium alloys between $30^{\circ} \mathrm{C}$ and $400^{\circ} \mathrm{C}, "$ Journal of the Institute of Metals, vol. 95, 1967.

[43] Z. L. Pan, I. G. Ritchie, and M. P. Puls, "The terminal solid solubility of hydrogen and deuterium in Zr-2.5Nb alloys," Journal of Nucelar Materials, vol. 228, p. 227-237, 1996.

[44] K. Une and S. Ishimoto, "Terminal Solid Solubility of Hydrogen in Unalloyed Zirconium by Differential Scanning Calorimetry," Journal of Nuclear Science and Technology, vol. 41, p. 949-952, 2004.

[45] C. Domain, R. Besson, and A. Legris, "Atomic-scale ab-initio study of the zr-h system: I. bulk properties," Acta Materialia, vol. 50, no. 13, pp. 3513-3526, 2002 .

[46] S. D. Kim, Y. Rhyim, J. S. Kim, and J. Yoon, "Characterization of zirconium hydrides in Zircaloy-4 cladding with respect to cooling rate," Journal of Nucelar Materials, vol. 465, p. 731-736, 2015.

[47] A. Fick, "Ueber diffusion," Annalen der Physik, vol. 170, pp. 59-86, 1855.

[48] S. Arrhenius, "Über die dissociationswärme und den einfluss der temperatur auf den dissociationsgrad der elektrolyte," Zeitschrift für Physikalische Chemie, vol. 4U, pp. $96-116,1889$.

[49] J. J. Kearns, "Diffusion coefficient of hydrogen in alpha zirconium, zircaloy-2 and zircaloy-4," Journal of Nuclear Materials, vol. 43, no. 3, pp. 330-338, 1972. 
[50] G. McRae, C. Coleman, H. Nordin, B. Leitch, and S. Hanlon, "Diffusivity of hydrogen isotopes in the alpha phase of zirconium alloys interpreted with the Einstein flux equation," Journal of Nuclear Materials, vol. 510, pp. 337-347, 2018.

[51] E. A. Gulbransen and K. F. Andrew, "Diffusion of hydrogen and deuteriun in high purity zirconium," Journal of the Electrochemical Society, vol. 101, no. 11, pp. 560-566, 1953.

[52] M. W. Mallett and W. M. Albrecht, "Low-pressure solubility and diffusion of hydrogen in zirconium," Journal of The Electrochemical Society, vol. 104, no. 3, pp. 142-146, 1957.

[53] M. Someno, "Solubility and diffusion of hydrogen in zirconium," Nippon Kinzoku Gakkaishi (Japan), vol. 24, pp. 249-253, 1960.

[54] A. Sawatzky, "The diffusion and solubility of hydrogen in the alpha phase of zircaloy-2," Journal of Nuclear Materials, vol. 2, no. 1, pp. 62-68, 1960.

[55] F. Mazzolai and J. Ryll-Nardzewsi, "An anelastic study of the diffusion coefficient of hydrogen in $\alpha$-zirconium," Journal of the Less-Common Metalls, vol. 49, pp. 323-327, 1976.

[56] S. Naito, "Kinetics of hydrogen absorption by $\alpha$-zirconium," The Journal of Chemical Physics, vol. 79, no. 6, pp. 3113-3120, 1983.

[57] R. K. Siripurapu, B. Szpunar, and J. A. Szpunar, "Molecular Dynamics Study of Hydrogen in $\alpha$-Zirconium," International Journal of Nuclear Energy, vol. 2014, 2014.

[58] Y. Zhang, C. Jiang, and X. Bai, "Anisotropic hydrogen diffusion in $\alpha$-Zr and Zircaloy predicted by accelerated kinetic Monte Carlo simulations," Scientific Reports, vol. 7, pp. 41033 1-13, 2017.

[59] H. Muta, Y. Etoh, Y. Ohishi, K. Kurosaki, and S. Yamanaka, "Ab initio study of hydrogen diffusion in zirconium oxide," Journal of Nuclear Science and Technology, vol. 49, no. 5, pp. 544-550, 2012.

[60] M. Christensen, W. Wolf, C. Freeman, E. Wimmer, R. B. Adamson, L. Hallstadius, P. E. Cantonwine, and E. V. Mader, " $\mathrm{H}$ in $\alpha$-zr and in zirconium hydrides: solubility, effect on dimensional changes, and the role of defects," Journal of Physics: Condensed Matter, vol. 27, pp. 025402 1-12, 2015.

[61] J. P. Pemsler, "Diffusion of oxygen in zirconium and its relation to oxidation and corrosion," Journal of the Electrochemical Society, vol. 105, pp. 315-322, 1958.

[62] D. Quataert and F. Coen-Porisini, "Utilization of the ion analyser for the study of oxygen diffusion in solids and its application to zirconium," Journal of $\mathrm{Nu}$ clear Materials, vol. 36, pp. 20-28, 1970. 
[63] D. David, G. Amsel, P. Boisot, and G. Beranger, "A study of the diffusion of oxygen in alpha-zirconium by means of nuclear microanalysis," Journal of the Electrochemical Society, vol. 122, pp. 388-396, 1975.

[64] I. G. Ritchie and A. Atrens, "The diffusion of oxygen in alpha-zirconium," Journal of Nuclear Materials, vol. 67, p. 254-264, 1977.

[65] C. O. D. González and E. A. García, "Determination of the diffusion coefficients of oxygen in zirconium by means of xps," Applied Surface Science, vol. 44, pp. 211-219, 1990.

[66] I. Grinberg, V. R. Cooper, and A. M. Rappe, "Oxide chemistry and local structure of $\mathrm{PbZr}_{x} \mathrm{Ti}_{1-x} \mathrm{O}_{3}$ studied by density-functional theory supercell calculations," Phys. Rev. B, vol. 69, no. 14, p. 144118, 2004.

[67] A. Gopejenko, S. Piskunov, and Y. F. Zhukovskii, "Ab initio modelling of the effects of varying $\mathrm{Zr}(\mathrm{Ti})$ concentrations on the atomic and electronic properties of stoichiometric PZT solid solutions," Comput. Theor. Chem., vol. 1104, pp. 56-60, 2017.

[68] B. Noheda, D. E. Cox, G. Shirane, L. E. Cross, and S.-E. Park, "A monoclinic ferroelectric phase in the $\mathrm{Pb}\left(\mathrm{Zr}_{1-x} \mathrm{Ti}_{x}\right) \mathrm{O}_{3}$ solid solution," Appl. Phys. Lett., vol. 74, no. 14, pp. 2059-2061, 1999.

[69] H. Mehrer, Diffusion in Solids: Fundamentals, Methods, Materials, DiffusionControlled Processes. Vienna, Austria: Springer-Verlag Berlin Heidelberg, 1 ed., 2007.

[70] A. Einstein, "Über die von der molekularkinetischen theorie der wärme geforderte bewegung von in ruhenden flüssigkeiten suspendierten teilchen," $A n$ nalen der Physik, vol. 322, no. 8, pp. 549-560, 1905.

[71] A. Einstein, "On the movement of small particles suspended in stationary liquids required by the molecular-kinetic theory of heat - on the random motion of particles suspended in a liquid and their relationship to diffusion," in The Swiss Years: Writings, 1900-1909 (English translation supplement) (J. Stachel, D. C. Cassidy, J. Renn, and R. Schulmann, eds.), vol. 2 of The Collected Papers of Albert Einstein, ch. 16, pp. 130-134, Princeton, New Jersey: Princeton University Press, 1990.

[72] H. Eyring, "The activated complex in chemical reactions," The Journal of Chemical Physics, vol. 3, pp. 107-115, 1935.

[73] J. T. Fermann and S. Auerbach, "Modeling proton mobility in acidic zeolite clusters: II. room temperature tunneling effects from semiclassical rate theory," J. Chem. Phys., vol. 112, pp. 6787-6794, 2000.

[74] W. Kohn and L. J. Sham, "Self-consisten equations includingexchange and correlation effects," Physical Review, vol. 140, p. A1133-A1138, 1965.

[75] P. Hohenberg and W. Kohn, "Inhomogeneous electron gas," Physical Review $B$, vol. 7, p. 1912-1919, 1973. 
[76] J. C. Slater, "A simplification of the hartree-fock method," Physical Review, vol. 81, p. 385-390, 1951.

[77] P. Østli, Density Functional Theory Studies of Precipitate Interfaces in Aluminium Alloys, with Focus on $\theta^{\prime}-\mathrm{Al}_{2} \mathrm{Cu}$. PhD thesis, Norwegian University of Science and Technology, 2016.

[78] S. Turgut, "Hartree-fock approximation." Lecture Notes, 2005.

[79] E. B. Tadmor and R. E. Miller, "Quantum mechanics of materials," in Modelling Materials - Continuum, Atomistic and Mutiscale Techniques, ch. 4, p. 153-236, Cambridge University Press, 2011.

[80] J. C. Cuevas, "Introduction to density functional theory." Lecture Notes, 2011.

[81] M. Born and R. Oppenheimer, "Zur quantentheorie de molekeln," Annalen der Physik, vol. 389, p. 457-484, 1927.

[82] D. R. Hartree, "The wave mechanics of an atom with a non-coulomb central field. part i. theory and methods," Mathematical Proceedings of the Cambridge Philosophical Society, vol. 24, pp. 89-110, 1928.

[83] V. Fock, "Näherungsmethode zur lösung des quantenmechanischen mehrkörperproblems," Zeitschrift für Physik, vol. 61, p. 126-148, 1930.

[84] L. H. Thomas, "The calculation of atomic fields," Mathematical Proceedings of the Cambridge Philosophical Society, vol. 23, p. 542-548, 1927.

[85] E. Fermi, "Un metodo statistico per la determinazione di alcune priorieta dell'atome," Rendicondi Accademia Nazionale de Lincei, vol. 32, p. 602-607, 1927.

[86] G. Kresse, M. Marsman, , and J. Furthmüller, Vienna Ab-initio Simulation Package VASP the GUIDE. 2014.

[87] G. Mills and H. Jónsson, "Quantum and thermal effects in $\mathrm{H}_{2}$ dissociative adsorption: Evaluation of free energy barriers in multidimensional quantum systems," Physical Review Letters, vol. 72, no. 7, pp. 1124-1127, 1994.

[88] G. Mills, H. Jónsson, and G. K. Schenter, "Reversible Work Transiton State Theory: Application to Dissociative Adsorption of Hydrogen," Surface Science, vol. 324, no. 2-3, pp. 305-337, 1995.

[89] H. Jónsson, G. Mills, and K. W. Jacobsen, "Nudged elastic band method for finding minimum energy paths of transitions," in Classical and Quantum Dynamics in Condensed Phase Simulations, ch. 16, pp. 385-404, World Scienific, 1998.

[90] G. Henkelman and H. Jónsson, "Improved tangent estimate in the nudged elastic band method for finding minimum energy paths and saddle points Improved tangent estimate in the nudged elastic band method for finding minimum energy paths and saddle points," Journal of Chemical Physics, vol. 113, pp. 9978-9985, 2000 
[91] G. Henkelman, B. P. Uberuaga, and H. Jónsson, "Climbing image nudged elastic band method for finding saddle points and minimum energy paths," Journal of Chemical Physics, vol. 113, no. 22, pp. 9901-9904, 2000.

[92] G. Kresse and J. Hafner, "Ab initio molecular dynamics for liquid metals," Physical Review B, vol. 47, no. 1, pp. 558-561, 1993.

[93] G. Kresse and J. Furthmüller, "Efficiency of ab-initio total energy calculations for metals and semiconductors using a plane-wave basis set," Computational Materials Science, vol. 6, no. 1, pp. 15-50, 1996.

[94] G. Kresse and J. Furthmüller, "Efficient iterative schemes for $<\mathrm{i}>$ ab initio $</ \mathrm{i}>$ total-energy calculations using a plane-wave basis set," Physical Review B, vol. 54, no. 16, pp. 11169-11186, 1996.

[95] "Nudged Elastic Band — Transition State Tools for VASP." http://theory . cm.utexas.edu/vtsttools/neb.html. Accessed: 2017 Jul. 08.

[96] A. F. Voter, "Introduction To the Kinetic Monte Carlo Method," in Radiation Effects in Solids (K. E. Sickafus, E. A. Kotomin, and B. P. Uberuaga, eds.), ch. 1, pp. 1-23, Dordrecht: Springer Netherlands, 2007.

[97] Y. Wang, W. Y. Chu, L. J. Qiao, and Y. J. Su, "Hydrogen-induced delayed fracture of PZT ceramics during dynamic charging under constant load," Materials Science and Engineering, vol. 98, no. 1, pp. 1-5, 2003.

[98] X. Peng, Y. J. Su, K. W. Gao, L. J. Qiao, and W. Y. Chu, "Hydrogen fissure in PZT ferroelectric ceramic," Materials Letters, vol. 58, no. 15, pp. 2073-2075, 2004.

[99] K. J. Alvine, M. Vijayakumar, M. E. Bowden, A. L. Schemer-Kohrn, and S. G. Pitman, "Hydrogen diffusion in lead zirconate titanate and barium titanate," Journal of Applied Physics, vol. 112, no. 4, pp. 043511 1-6, 2012.

[100] Y. Gou, Y. Li, Y. Liu, H. Chen, and S. Ying, "Evaluation of a delayed hydride cracking in zr-2.5nb candu and rbmk pressure tubes," Materials and Design, vol. 30, no. 4, pp. 1231-1235, 2009.

[101] International Atomic Energy Agency, Delayed hydride cracking in zirconium alloys in pressure tube nuclear reactors. Vienna: International Atomic Energy Agency, 2004.

[102] R. N. Singh, T. K. Sinha, and B. P. Kashyap, "Delayed hydride cracking in Zr-2 . 5Nb pressure tube material," Journal of Nuclear Materials, vol. 304, pp. 189-203, 2002.

[103] L. A. Simpson and C. D. Cann, "Fracture toughness of zirconium hydride and its influence on the crack resistance of zirconium alloys," Journal of Nuclear Materials, vol. 87, pp. 303-316, 1979.

[104] G. McRae, C. Coleman, and H. Nordin, "Diffusivity of hydrogen isotopes in zirconium alloys interpreted with the einstein flux equation," 2017. Presentation in the Canadian Material Science Conference, Ottawa ON. 
[105] E. S. Fisher and C. J. Renkin, "Single-crystal elastic moduli and the hcp - bcc transformation in ti, zr, and hf," Physical Review, vol. 135, no. 2A, pp. A482A494, 1964.

[106] G. Vérité, F. Willaime, and C. C. Fu, "Anisotropy of the vacancy migration in $\mathrm{Ti}, \mathrm{Zr}$ and Hf hexagonal close-packed metals from first principles," Solid State Phenomena, vol. 129, pp. 75-81, 2007.

[107] J. P. Perdew, K. Burke, and M. Ernzerhof, "Generalized Gradient Approximation Made Simple," Physical Review Letters, vol. 77, no. 18, pp. 3865-3868, 1996.

[108] J. B. Bates, J. C. Wang, and R. A. Perkins, "Mechanisms for hydrogen diffusion in tio 2 ," Physical Review B, vol. 19, no. 8, pp. 4130-4139, 1979.

[109] S. Fujita and A. Garcia, "Theory of hydrogen diffusion in metals. quantum isotope effects," J. Phys. Chem. Solid., vol. 52, no. 2, pp. 351-355, 1991.

[110] E. M. McIntosh, K. T. Wikfeldt, J. Ellis, A. Michaelides, and W. Allison, "Quantum effects in the diffusion of hydrogen on ru(0001)," J. Phys. Chem. Lett., vol. 4, no. 9, pp. 1565-1569, 2013.

[111] A. Tezuka, H. Wang, H. Ogawa, and T. Ikeshoji, "Potential energy surface and hopping path for hydrogen in lani 5 ," Phys. Rev. B, vol. 81, no. 13, p. 134304, 2010 .

[112] G. Henkelman, A. Arnaldsson, and H. Jónsson, "Theoretical calculations of $\mathrm{ch}_{4}$ and $\mathrm{h}_{2}$ associative desorption from ni(111): Could subsurface hydrogen play an important role?," J. Chem. Phys., vol. 124, no. 4, p. 044706, 2006.

[113] G. B. Skinner and H. L. Johnston, "Thermal expansion of zirconium between $298 \mathrm{k}$ and 1600 k," J. Chem. Phys., vol. 21, no. 8, pp. 1383-1384, 1953.

[114] T. Hickel, B. Grabowski, F. Körmann, and J. Neugebauer, "Advancing density functional theory to finite temperatures: Methods and applications in steel design," J. Phys. Condens. Matter, vol. 24, no. 5, p. 053202, 2012.

[115] N. Mermin, "Thermal properties of the inhomogeneous electron gas," Phys. Rev., vol. 137, no. 5A, pp. A1441-1443, 1965.

[116] A. Pribram-Jones, P. E. Grabowski, and K. Burke, "Thermal density functional theory: Time-dependent linear response and approximate functionals from the fluctuation-dissipation theorem," Phys. Rev. Lett., vol. 116, no. 23, p. 233001, 2016 .

[117] K. Momma and F. Izumi, "Vesta 3 for three-dimensional visualization of crystal, volumetric and morphology data," Journal of Applied Crystallography, vol. 44, pp. 1272-1276, 2011.

[118] S. Banerjee, "Nuclear applications: Zirconium alloys," in Encyclopedia of Materials: Science and Technology (K. Buschow, R. Cahn, M. Flemings, B. Ilschner, E. Kramer, S. Mahajan, and P. Veyssiere, eds.), pp. 6287-6299, Elsevier, 2 ed., 2001 . 
[119] D. Féron, "Overview of nuclear materials and nuclear corrosion science and engineering," in Nuclear Corrosion Science and Engineering (D. Féron, ed.), ch. 2, pp. 31-56, Woodhead Publishing, 2012.

[120] G. McRae, C. Coleman, and B. Leitch, "The first step for delayed hydride cracking in zirconium alloys," J. Nucl. Mater., vol. 396, pp. 130-143, 2010.

[121] M. De Las Heras, S. Parodi, L. Ponzoni, J. Mieza, S. Müller, S. Alcantar, and G. Domizzi, "Effect of thermal cycles on delayed hydride cracking in Zr-2.5Nb alloy," J. Nucl. Mater., vol. 509, pp. 600-612, 2018.

[122] T. N. Murty, R. Singh, and P. Ståhle, "Delayed hydride cracking of Zr-2.5\%nb pressure tube material due to partially constrained precipitates," J. Nucl. Mater., vol. 513, pp. 129-142, 2019.

[123] C. Roy, "An experiment to clarify the effect of dissolved oxygen on the terminal solubility of hydrogen in zirconium," Journal of Nuclear Materials, vol. 13, pp. 275-277, 1964.

[124] B. Bhatia and D. S. Sholl, "Quantitative assessment of hydrogen diffusion by activated hopping and quantum tunneling in ordered intermetallics," Phys. Rev. $B$, vol. 72, p. 224302, 2005.

[125] B. Puchala, M. L. Falk, and K. Garikipati, "An energy basin finding algorithm for kinetic monte carlo acceleration," J. Chem. Phys., vol. 132, p. 134104, 2010.

[126] M. Liyanage, R. E. Miller, and R. K. N. D. Rajapakse, "Multi-scale approach for determining hydrogen diffusivity in zirconium," Model. Simul. Mater. Sci. Eng., vol. 26, p. 085002, 2018.

[127] A. Denis, E. Moyano, and E. A. Garcia, "Model to simulate high temperature oxidation kinetics of zircaloy-4: Parabolic and linear behaviour," J. Nucl. Mater., vol. 110, pp. 11-19, 1982.

[128] R. Bordoni and A. Olmedo, "Oxide thickness measurements and characterization of two zr-2.5nb pressure tubes of embalse nuclear power plant," Acta Microsc., vol. 20, pp. 10-20, 2011.

[129] C. J. Rosa and H. W. C., "Oxygen-ion diffusivity in hypostoichiometric zirconium dioxide," J. Nucl. Mater., vol. 27, pp. 12-20, 1968.

[130] L. Zhang, L.-Y. Chen, C. Zhao, Y. Liu, and Z. Lai-Chang, "Calculation of oxygen diffusion coefficients in oxide films formed on low-temperature annealed zr alloys and their related corrosion behavior," Metals., vol. 9, p. 850, 2019.

[131] M. Liyanage, R. E. Miller, and R. K. N. D. Rajapakse, "Denuded zones in zirconium pressure vessels: Oxygen's role examined via multi-scale diffusion model." Manuscript submitted for publication, 2020.

[132] P. Sofronis and R. McMeeking, "Numerical analysis of hydrogen transport near a blunting crack tip," Journal of the Mechanics and Physics of Solids, vol. 37, no. 3, pp. $317-350,1989$. 
[133] F. X. Li, R. K. N. D. Rajapakse, D. Mumford, and M. Gadala, "Quasi-static thermo-electro-mechanical behaviour of piezoelectric stack actuators," Smart Mater. Struct., vol. 17, no. 1, p. 015049, 2018.

[134] P. Cotterill, "The hydrogen embrittlement of metals," Prog. Mater. Sci., vol. 9, no. 4, pp. 205-250, 1961.

[135] H. C. Rogers, "Hydrogen embrittlement of metals," Science, vol. 10, no. 3819, pp. 1057-1064, 1968.

[136] M. Louthan Jr., G. Caskey Jr., J. Donovan, and D. Rawl Jr., "Hydrogen embrittlement of metals - fragilisation des métaux par l' hydrogéne wasserstoffversprödung in metallen," Mater. Sci. Eng., vol. 10, pp. 357-368, 1972.

[137] F. P. Fengler, M. Pesic, S. Starschich, T. Schneller, U. Bottger, T. Schenk, M. H. Park, T. Mikolajick, and U. Schroeder, 2016 46th European Solid-State Device Research Conference (ESSDERC), pp. 369-372. Lausanne, Switzerland: IEEE, 2016.

[138] N. Jalili, J. Wagner, and M. Dadfarnia, "A piezoelectric driven ratchet actuator mechanism with application to automotive engine valves," Mechatronics, vol. 13, pp. 933-956, 2003.

[139] K. Kushida-Abdelghafar, H. Miki, K. Torii, and Y. Fujisaki, "Electrode-induced degradation of $\mathrm{Pb}\left(\mathrm{Zr}_{x} \mathrm{Ti}_{1-x}\right) \mathrm{O}_{3}$ (PZT) polarization hysteresis characteristics in Pt/PZT/Pt ferroelectric thin-film capacitors," Appl. Phys. Lett., vol. 69, no. 21, pp. 3188-3190, 1996.

[140] Y. Shimakawa and Y. Kubo, "Degradation of ferroelectric $\mathrm{Pb}(\mathrm{Zr}, \mathrm{Ti}) \mathrm{O}_{3}$ under reducing conditions," Appl. Phys. Lett., vol. 77, no. 16, pp. 2590-2592, 2000.

[141] Q. Wang and F. Li, "A low-working-field $(2 \mathrm{kV} / \mathrm{mm})$, large-strain $(>0.5 \%)$ piezoelectric multilayer actuator based on periodically orthogonal poled PZT ceramics," Sensor. Actuat. A: Phys., vol. 272, pp. 212-216, 2018.

[142] H. Zhao, Z.-R. Qu, H.-Y. Ye, and R.-G. Xiong, "In situ hydrothermal synthesis of tetrazole coordination polymers with interesting physical properties," Chem. Soc. Rev., vol. 37, no. 1, pp. 84-100, 2008.

[143] B. Noheda, J. A. Gonzalo, L. E. Cross, R. Guo, S.-E. Park, D. E. Cox, and G. Shirane, "Tetragonal-to-monoclinic phase transition in a ferroelectric perovskite: The structureof $\mathrm{PbZr}_{0.52} \mathrm{Ti}_{0.48} \mathrm{O}_{3}, "$ Phys. Rev. B, vol. 61, no. 13, pp. 8687-8695, 2000.

[144] B. Jaffe, W. R. Cook, and H. L. Jaffe, Piezoelectric ceramics. London and New York: Academic Press, 1971.

[145] J. Frantti, S. Ivanov, S. Eriksson, H. Rundlöf, V. Lantto, J. Lappalainen, and M. Kakihana, "Phase transitions of $\mathrm{Pb}\left(\mathrm{Zr}_{x} \mathrm{Ti}_{1-x}\right) \mathrm{O}_{3}$ ceramics," Phys. Rev. B, vol. 66, no. 6, p. 064108, 2002. 
[146] N. Zhang, H. Yokota, A. M. Glazer, and P. A. Thomas, "Neutron powder diffraction refinement of $\mathrm{PbZr}_{1-x} \mathrm{Ti}_{x} \mathrm{O}_{3}, "$ Acta Crystallogr. B, vol. 67, no. 5, pp. 386-398, 2011.

[147] C. L. Jia, J. R. Contreras, U. Poppe, H. Kohlstedt, R. Waser, and K. Urban, "Lattice strain and lattice expansion of the $\mathrm{SrRuO}_{3}$ layers in $\mathrm{SrRuO}_{3} / \mathrm{PbZr}_{0.52} \mathrm{Ti}_{0.48} \mathrm{O}_{3} / \mathrm{SrRuO}_{3}$ multilayer thin films," J. Appl. Phys., vol. 92, no. 1, pp. 101-105, 2002.

[148] G. Sághi-szabó, R. E. Cohen, and H. Krakauer, "First-principles study of piezoelectricity in tetragonal $\mathrm{PbTiO}_{3}$ and $\mathrm{PbZr}_{1 / 2} \mathrm{Ti}_{1 / 2} \mathrm{O}_{3}$," Phys. Rev. B, vol. 59, no. 20, pp. 12771-12776, 1999.

[149] M. Wu, H. Huang, W. Chu, L. Guo, L. Qiao, J. Xu, and T.-Y. Zhang, "Tuning the Ferroelectric and Piezoelectric Properties of $0.91 \mathrm{~Pb}\left(\mathrm{Zn}_{1 / 3} \mathrm{Nb}_{2 / 3}\right) \mathrm{O}_{3^{-}}$ $0.09 \mathrm{PbTiO}_{3}$ Single Crystals and Lead Zirconate Titanate Ceramics by Doping Hydrogen," J. Phys. Chem. C, vol. 114, no. 21, pp. 9955-9960, 2010.

[150] K. Xiong and J. Robertson, "Hydrogen-induced defects and degradation in oxide ferroelectrics," Appl. Phys. Lett., vol. 85, no. 13, pp. 2577-2579, 2004.

[151] X.-H. Guo, S.-Q. Shi, and L.-J. Qiao, "Simulation of Hydrogen Diffusion and Initiation of Hydrogen-Induced Cracking in PZT Ferroelectric Ceramics Using a Phase Field Model," J. Am. Ceram. Soc., vol. 90, no. 9, pp. 2868-2872, 2007.

[152] Y. Fujisaki, K. Kushida-Abdelghafar, Y. Shimamoto, and H. Miki, "The effects of the catalytic nature of capacitor electrodes on the degradation of ferroelectric $\mathrm{Pb}(\mathrm{Zr}, \mathrm{Ti}) \mathrm{O}_{3}$ thin films during reductive ambient annealing," J. Appl. Phys., vol. 82, no. 1, pp. 341-344, 1997.

[153] J. P. Han and T. P. Ma, "Electrode dependence of hydrogen-induced degradation in ferroelectric $\mathrm{Pb}(\mathrm{Zr}, \mathrm{Ti}) \mathrm{O}_{3}$ and $\mathrm{SrBi}_{2} \mathrm{Ta}_{2} \mathrm{O}_{9}$ thin films," Appl. Phys. Lett., vol. 71, no. 9, pp. 1267-1269, 1997.

[154] J. K. Lee, Y. Park, and I. Chung, "Investigation of hydrogen-induced degradation in $\mathrm{Pb}\left(\mathrm{Zr}-x \mathrm{Ti}_{1-x}\right) \mathrm{O}_{3}$ thin film capacitors for the application of memory devices," J. Appl. Phys., vol. 92, no. 5, pp. 2724-2728, 2002.

[155] A. Shafiei, C. Oprea, A. Alfantazi, and T. Troczynski, "In situ monitoring of the effects of hydrogen on $\mathrm{Pb}(\mathrm{Zr}, \mathrm{Ti}) \mathrm{O}_{3}$ structure," J. Appl. Phys., vol. 109, no. 11, p. 114108, 2011.

[156] N. R. Rajopadhye, S. V. Bhoraskar, S. Badrinarayan, and S. A. P. B., "Photoacoustic and X-ray photoelectron spectroscopic studies in reduced lead zirconate titanate ceramics," J. Mater. Sc., vol. 23, no. 7, pp. 2631-2636, 1988.

[157] J.-M. Koo, T. Kim, and J. Kim, "Hydrogen induced degradation ]phenomena of pzt ferroelectric capacitors," in Proc. 2000 12th IEEE Int. Symp. Appl. Ferr. (S. K. Streiffer, B. J. Gibbons, T. Tsurumi, and M. A. A. E. Zurbuchen, eds.), vol. 2, (Honolulu, HI, USA), pp. 591-594, Institute of Electrical and Electronics Engineers, Inc., 2000. 
[158] H. Y. Huang, W. Y. Chu, Y. J. Su, K. W. Gao, J. X. Li, and L. J. Qiao, "Hydrogen-induced semiconductor transformation of lead zirconate titanate ferroelectric ceramics," J. Am. Ceram. Society, vol. 90, no. 7, pp. 2062-2066, 2007.

[159] W. Chen, L. Li, Y. Wang, and Z. Gui, "Effects of electrochemical hydrogen charging on lead-based relaxor ferroelectric multilayer ceramic capacitors," $J$. Mater. Res., vol. 13, no. 05, pp. 1110-1112, 1998.

[160] D. Houde, Y. Lépine, C. Pépin, S. Jandl, and J. L. Brebner, "High-resolution infrared spectroscopy of hydrogen impurities in strontium titanate," Phys. Rev. $B$, vol. 35, no. 10, pp. 4948-4953, 1987.

[161] C. H. Park and D. J. Chadi, "Effect of interstitial hydrogen impurities on ferroelectric polarization in $\mathrm{PbTiO}_{3}$," Phys. Rev. Lett., vol. 84, no. 20, pp. 4717-4720, 2000 .

[162] J. P. Perdew, A. Ruzsinszky, G. I. Csonka, O. A. Vydrov, G. E. Scuseria, L. A. Constantin, X. Zhou, and K. Burke, "Restoring the Density-Gradient Expansion for Exchange in Solids and Surfaces," Phys. Rev. Lett., vol. 100, no. 13, p. 136406, 2008.

[163] D. Berlincourt and H. H. A. Krueger, "Domain processes in lead titanate zirconate and barium titanate ceramics," J. Appl. Phys., vol. 30, no. 11, pp. 18401810, 1959. 


\section{Appendix A}

\section{Semi-Classical Harmonic Transition State Theory}

SC-HTST formulated by Fermann and Auerbach [1] only requires the activation energy and vibration frequencies of initial and transition state. Climbing image NEB was used to find the activation energy and the central difference method was used to determine the vibration frequencies by decoupling the $\mathrm{H}$ atoms from the remaining atoms. The hopping rate from SC-HTST $\left(\Gamma^{S C-H T S T}\right)$ can be calculated as

$$
\Gamma^{S C-H T S T}=\Gamma^{H T S T} k(T)
$$

where $\Gamma^{H T S T}$ is the harmonic transition state theory hopping rate and $k(T)$ is the quantum tunneling correction. $\Gamma^{H T S T}$ can be calculated as

$$
\Gamma^{H T S T}=\frac{\prod_{i=1}^{3} v_{i} f\left(h v_{i} / 2 k_{B} T\right)}{\prod_{j=1}^{2} v_{j}^{T S} f\left(h v_{j}^{T S} / 2 k_{B} T\right)} \exp \left(-E_{a} / k_{B} T\right)
$$

where, $h$ is Planck's constant, $v_{i}$ and $v_{j}^{T S}$ are the vibration frequencies associated with the initial state and the transition state respectively, $k_{B}$ is the Boltzmann constant, $T$ is the temperature, and the function $f(x)=\sinh (x) / x$. This already includes the effect of zero-point energy (ZPE) on the classical activation energy $E_{a}$. The quantum tunneling is a temperature dependant multiplier to be determined as

$$
k(T)=\frac{\exp \left(E_{Z P} / k_{B} T\right)}{1+\exp \left(2 \theta_{0}\right)}+\frac{1}{2} \int_{-\infty}^{\theta_{0}} \operatorname{sech}^{2} \theta \exp \left(\frac{h v_{ \pm} \theta}{\pi k_{B} T}\right) d \theta
$$


where $\theta_{0}=\left(\pi E_{Z P}\right) /\left(h v_{ \pm}\right)$and $v_{ \pm}$is the imaginary vibration frequency at the transition state. $E_{Z P}$ is the ZPE corrected activation energy to be calculated as

$$
E_{Z P}=E_{a}-\frac{1}{2} \sum_{i=1}^{3} h v_{i}+\frac{1}{2} \sum_{j=1}^{2} h v_{j}^{T S}
$$

\section{Bibliography}

[1] J. T. Fermann and S. Auerbach, "Modeling proton mobility in acidic zeolite clusters: II. room temperature tunneling effects from semiclassical rate theory," $J$. Chem. Phys., vol. 112, pp. 6787-6794, 2000. 


\section{Appendix B}

\section{Absorbing Markov Chains}

As discussed, one major issue faced during KMC simulation of $\mathrm{Zr}$ and other hcp metals, is the back and forth movement between nearest neighbor tetrahedral sites. While there are several methods available to solve this problem, we followed the mean rate method shown in Puchala et al. [1] where the tetrahedral sites are considered as transient states, and the octahedral sites are considered as absorbing states in a Markov chain for each pseudo-energy basin. Using this method, the rate of escape from the pseudo-energy basin to its nearest neighbor sites are determined.

Initially the transition probability matrix $\overline{\bar{T}}$ is created with entries

$$
T_{i j}=\frac{\Gamma_{i \rightarrow j}}{\sum_{k} \Gamma_{i \rightarrow k}}
$$

where $\Gamma_{i \rightarrow j}$ is the hopping rate from transient state $i$ to transient state $j$ and $k$ are all the transient and absorbing states available to transition from state $i$. This can be also written as

$$
T_{i j}=\tau_{i}^{1} \Gamma_{i \rightarrow j}
$$

where $\tau_{i}^{1}=\left(\sum_{k} \Gamma_{i \rightarrow k}\right)^{-1}$ is the mean residence time at state $i$. The probability of still being in each transient state after $m$ jumps, the occupational probability vector can be determined as

$$
\bar{\Theta}(m)=\overline{\bar{T}}^{m} \tilde{\Theta}(0)
$$

where $\bar{\Theta}(0)$ gives the occupancy at the start with entries,

$$
\Theta_{i}(0)= \begin{cases}1 & \text { if } i=s_{\text {init }} \\ 0 & \text { otherwise }\end{cases}
$$


with $s_{\text {init }}$ being the initial occupancy site. By summing the occupational probability over all $m$ we get the total occupational probabilty vector as

$$
\bar{\Theta}^{t o t}=\sum_{m=0}^{\infty} \overline{\bar{T}}^{m} \bar{\Theta}(0)=(I-\overline{\bar{T}})^{-1} \Theta(0)
$$

From this, the mean residence time at each transient state can be determined as

$$
\tau_{i}=\tau_{i}^{1} \Theta_{i}^{t o t}
$$

The rates for escaping into each of the absorbing state can be modified via

$$
\left\langle\Gamma_{i \rightarrow j}\right\rangle=\frac{\tau_{i}}{\sum_{k} \tau_{k}} \Gamma_{i \rightarrow j}
$$

with $k$ summed over all transient states.

For pseudo-energy basins comprising two nearest neighbor tetrahedral sites, the initial occupancy site can be either of the two. In the solutions it didn't give an appreciable difference to the rates based on the initial occupancy location. Hence, we calculated the modified rates for both cases and then took the average rate to determine the rates to be used in KMC simulations.

To check the validity of this method, we looked at the variation of average basin exit time and the probability of taking each of the escape path for the regular KMC, accelerated KMC, and the analytic solution by looking at a pseudo basin formed by $T_{2,1}$ and $T_{2,2}$ sites for $100,000 \mathrm{KMC}$ runs. A $\mathrm{H}$ atom in $T_{2,1}$ has the possibility to exit to one $O_{3,1}$ site and two $O_{2,1}$ sites, while $T_{2,2}$ has the possibility to exit to one $O_{3,2}$ site and two $O_{2,2}$ sites. In Fig. B.1 and Fig. B.2 we can see that the three methods gives very similar results, validating this method.

\section{Bibliography}

[1] B. Puchala, M. L. Falk, and K. Garikipati, "An energy basin finding algorithm for kinetic monte carlo acceleration," J. Chem. Phys., vol. 132, p. 134104, 2010. 


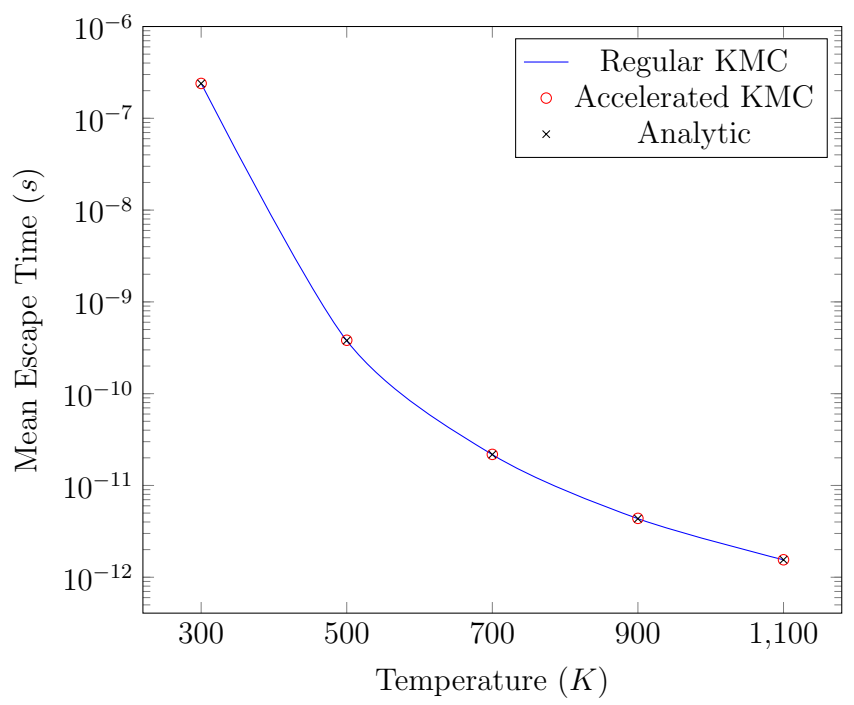

Figure B.1: Mean escape time from the pseudo-energy basin formed by nearest neighbour $T_{2,1}$ and $T_{2,2}$ sites

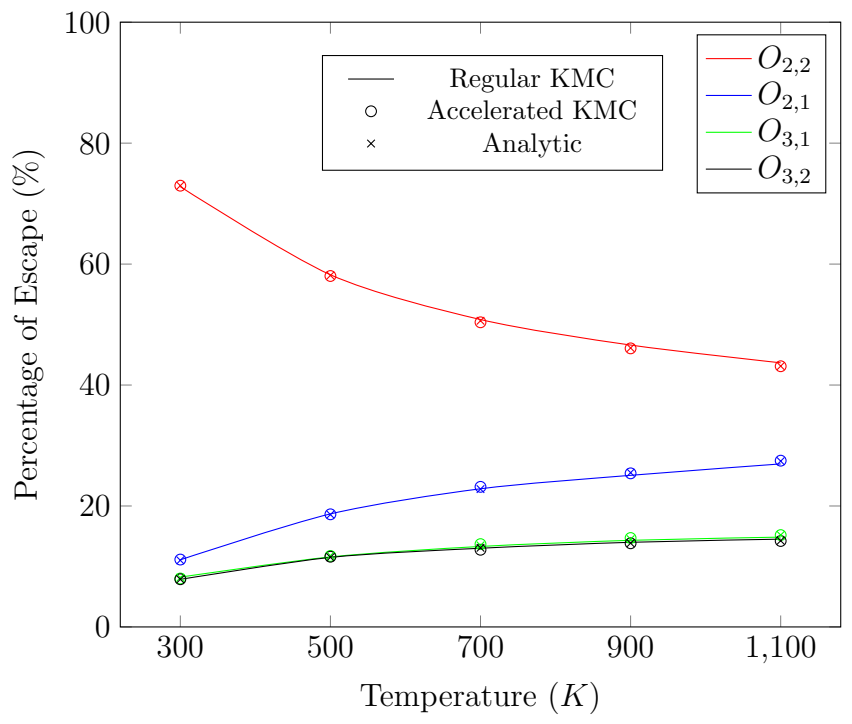

Figure B.2: $\quad$ Probability of exiting along possible paths from the pseudo-energy basin formed by nearest neighbour $T_{2,1}$ and $T_{2,2}$ sites. Solid lines shows the results from regular $\mathrm{KMC}$, the o shows the results from accelerated KMC, and the $\times$ shows the analytic solution. Note that the two lowest lines fall virtually atop each other 